\begin{abstract}
Title of dissertation: $\quad$ RBCC ENGINE-AIRFRAME INTEGRATION ON AN OSCULATING CONE WAVERIDER VEHICLE

Timothy F. O'Brien, Doctor of Philosophy, 2001

Dissertation directed by: Professor Mark J. Lewis

Department of Aerospace Engineering
\end{abstract}

An analytical vehicle study is performed that integrates a rocket-based combined cycle engine with an osculating cones waverider-based fuselage. The integration of the two concepts brings about an interesting design challenge: predicting the aerodynamic performance of a high-speed fuselage design across the full range of Mach numbers from take-off to orbit that a rocket-based combinedcycle engine will operate. The aerodynamic performance of this class of vehicles is analyzed for on- and off-design Mach numbers and angles of attack. Analytical aerodynamic models are developed for the off-design behavior of both the fuselage of the vehicle and the engine. These models are combined to predict the powered performance of this class of vehicle along a trajectory. The models developed are rapid enough that they may be applied to initial design studies, optimization algorithms, or trajectory analyses.

The aerodynamic model for the fuselage is based on the tangent-wedge, tangent-cone, and shock-expansion theories for hypersonic flow, and the lin- 
earized, small perturbation, velocity potential equations for supersonic and transonic flow. Each model is validated with numerical solutions for an example Mach 12 vehicle design. The results show an accurate prediction of the trends in lift and drag of the vehicle fuselage across a range of Mach numbers between 0.4 and 15 .

The aerodynamic engine model is based on Prandtl-Meyer flow and the oblique shock relations for the internal compression system, and quasi-onedimensional flow (including finite-rate chemistry) for the combustor flowfield. The strut-based compression model is validated with numerical solutions for a range of Mach numbers between 2.5 and 6. The combustor flowfield model is validated by comparison to two experimental hydrogen-fueled scramjet engines.

The results showed that this class of geometry generates very little lift at lowspeeds (below Mach 3) and will require lift augmentation. The transonic drag rise is modeled analytically and numerically, with maximum inviscid drag coefficient occurring at Mach 1.2. Engine integration has a large effect on off-design behavior, including maximum lift-to-drag ratio and zero lift angle of attack. 


\title{
RBCC ENGINE-AIRFRAME INTEGRATION ON AN OSCULATING CONE WAVERIDER VEHICLE
}

\author{
by \\ Timothy F. O'Brien
}

Dissertation submitted to the Faculty of the Graduate School of the University of Maryland, College Park in partial fulfillment of the requirements for the degree of Doctor of Philosophy 2001

Advisory Committee:

Professor Mark J. Lewis, Chairman/Advisor Associate Professor James D. Baeder Professor Ashwani K. Gupta Visiting Professor Robert H. Korkegi Associate Professor Kenneth Yu 
(C) Copyright by

Timothy F. O'Brien

2001 
(c) Copyright by

Timothy F. O'Brien

2001 


\section{DEDICATION}

To my grandparents: Francis and Eleanor O'Brien \&

Henry and Helen Blum 


\section{ACKNOWLEDGEMENTS}

I would like to begin by thanking my advisor Dr. Mark J. Lewis. His advice, guidance, and support were instrumental in the completion of my dissertation. I would also like to thank my committee members Dr. James D. Baedor, Dr. Aslwani K. Gupta, Dr. Robert H. Korkegi, and Dr. Kenneth Yu for their time, advice, constructive criticism, and insigltt into my work.

Appreciation goes out to NASA for funding the Conter for Hypersonics Education and Research at the University of Maryland (with Don Palac as technical monitor). Thanks also go to Lockheed Martin Skunk Works for their funding during the initial phases of my research (with Dr. Robert Boyd and Dr. Mary Hudson as technical monitors). The opportunities that both NASA and Lockhced Martin provided have enriched me both professionally and personally.

I would also like to thank all of my co-workers in the toolshed, past and prescent, for keeping things entertaining and providing incentive to leave this place. Special thanks go to Dr. Ryan Starkcy for his assistance with the combustor code and CFD solutions as well as gencral insight, into my work and dissertation, James Reuster for his 
uncanny knowledge of all things CFD and wisdom into my dissertaLion, Jee Su Chang for the initial combustor model, and Lael R.udd for his assistance with the trajectory model. Thlanks also go to Bryan IIill for working his "magic" and putting up with my pestering.

I'd also like to thank my cntire family for dealing with me being in school my entire life. Their encouragement and enthusiasm spurred me on when I lad no motivation.

Finally, I'd like to thank my wonderful wifn Sue for being my "sugar momma" for all theso years. In my darkest hours, she pushed me on, and I can honestly say that this work would not be possible without her unfailing support. I only hope that in the years to come, I can return the favor. 


\section{TABLE OF CONTENTS}

List of Tiables

1 Introduction

1.1 Motivation ...................... 1

1.2 Previous Work . . . . . . . . . . . . . . . . . 3

1.2.1 Waverider Geometrics ............... 3

1.2.2 Waverider-Based Engine-Airframe Integration . . . . . . . 5

1.2.3 Off-Design Wavorider Performance . . . . . . . . . 8

1.2.4 Rocket-Based Combined-Cycle Engines . . . . . . . . 13

1.3 Present Work . . . . . . . . . . . . . . . 17

2 Airframe Geometry Definition $\quad 19$

2.1 Waverider Forebody . . . . . . . . . . . . . . . 19

2.1 .1 Conical Flow Solution ............... 21 
2.1.2 Osculating Cones Waverider Mcthod . . . . . . . 23

2.1 .3 Streamine Tracing . . . . . . . . . . . . 24

2.1.4 Examples Using the Osculating Cones Waverider Method . 26

2.2 Enginc Inlet $\ldots \ldots \ldots \ldots \ldots \ldots$

2.3 Nozzle . . . . . . . . . . . . . . . . . . 32

2.4 Upper and Lower Aft Body Sections $\ldots \ldots \ldots \ldots$

2.5 Side Walis and Engine Cowl . . . . . . . . . . 35

2.6 Airframe Properties . . . . . . . . . . . . . . . . 37

2.6 .1 Volume Calculation . . . . . . . . . . . . 37

2.6.2 Friction Coefficicnt and Shear Stress _... . . . 38

2.6 .3 Heat Transfer . . . . . . . . . . . . . . . 39

2.6 .4 Vehicle Mass . . . . . . . . . . . . . . 40

2.6.5 Area and Force Calculation . . . . . . . . . . . 11

2.6 .6 Centrifugal Lift $\ldots \ldots \ldots$. . . . . . . . . . . 43

2.6 .7 Trajectory Model . . . . . . . . . . . . . . 43

3 R.BCC Engine Model $\quad 45$

3.1 Supersonic Strut Interaction Model . . . . . . . . . . . 46

3.1 .1 Validation . . . . . . . . . . . . . 51

3.2 Supersonic Combustion Flowfield Model . . . . . . . . . 54

3.2 .1 Continuity . . . . . . . . . . . . 55

3.2 .2 Momentum . . . . . . . . . . . . 56

3.2 .3 Equation of State . . . . . . . . . . 56

3.2 .4 Mixture Molecular Woight . . . . . . . . . . 57

3.2 .5 Species Conservation . . . . . . . . . . . 57

3.2 .6 Energy . . . . . . . . . . . . . . . . 59 
3.2 .7 Solution Methodology . . . . . . . . . . . 62

3.2 .8 Scramjet Mode Engine Validation . . . . . . . . . 65

3.2.9 Sensitivity of Engine Model to Fuel Injection Profile . . . . 70

3.2.10 Example Rocket-Based Combined Cycle Engine Solution in Scramjet Mode . . . . . . . . . . . . . . 73

3.3 Ramjet Mode . . . . . . . . . . . . . . . . . . . 77

4 Transonic Off-Design Aerodynamic Performance 83

4.1 Two-Dimensional Flow Solution $\ldots \ldots \ldots$. . . . . . 83

4.1 .1 Supersonic Solution . . . . . . . . . . . . . . 85

4.1 .2 Subsonic Solution . . . . . . . . . . . . . 86

4.1 .3 Near-Sonic Solution . . . . . . . . . . . . . . . . . . 87

4.2 Axisymmetric Flow Solution . . . . . . . . . . . . 88

4.2 .1 Supersonic Solution . . . . . . . . . . . . . . . . . 89

4.2 .2 Subsonic Solution . . . . . . . . . . . . . . . 9 91

4.2 .3 Near-Sonic Solution . . . . . . . . . . . . . . . . . 91

4.3 Application of Local Linearization Technique to Prescribed Geometries . . . . . . . . . . . . . . 92 4.3.1 Two-Dimensional Results . . . . . . . . . . . . 95 4.3 .2 Axisymmetric Results . . . . . . . . . . . . 108

4.4 Application to Full Vehicle Geometry . . . . . . . . . . . 118

4.5 Validation . . . . . . . . . . . . . . 126

5 Hypersonic Off-Design Aerodynamic Performance 140

5.1 Forebody . . . . . . . . . . . . . . . . 140

5.2 Fuselage . . . . . . . . . . . . . . . . 145 
5.3 Validation . . . . . . . . . . . . . 146

5.3 .1 On-Design Conditions . . . . . . . . . . . . 146

5.3 .2 Off-Design Conditions $\ldots \ldots \ldots \ldots \ldots$

5.4 Off-Design Engine Cowl . . . . . . . . . . . . 158

6 Supersonic Off-Design Aerodynamic Performance 160

6.1 Aerodynatnic Model . . . . . . . . . . . . . . . 160

6.2 Validation . . . . . . . . . . . . . . 162

6.3 Off-Design Engine Cowl . . . . . . . . . . . . . 169

7 Resuits 170

7.1 Unpowered Fusclage Performance . . . . . . . . . . 170

7.1 .1 Hypersonic Perfornance . . . . . . . . . . . . 170

7.1.2 Supersonic Performance . . . . . . . . . . . . . . . . 179

7.1.3 Transonic Performance . . . . . . . . . . . . 186

7.1.4 Blending of Methods . . . . . . . . . . . . . . . 192

7.2 Enginc-Airframe Integrated Performance . . . . . . . . . . 204

7.2 .1 Scramjet Integration $\ldots \ldots \ldots$. . . . . . . . . . 204

7.2 .2 Ramjet Integration $\ldots \ldots \ldots . \ldots . \ldots 210$

7.3 Scramjet-Powered Trajectory Performance . . . . . . . . 212

8 Summary and Conclusions 216

9 Future Work 220

9.1 Vehicle Definition . . . . . . . . . . . . . . 220

9.2 Off-Design Aerodynamic Models . . . . . . . . . . 22I

9.3 RBCC Engine Model . . . . . . . . . . . . . . 223 
A Secant Method

B Hydrogen/Air Reaction Mechanism 


\section{LIST OF TABLES}

3.1 Sensitivity of combustor properties to mixing profile. . . . . . 72

4.1 Forces on lower surface of the vehicle $\left(\mathrm{m}^{2}\right) \ldots \ldots \ldots \ldots$

4.2 Forces on upper surface of the vehicle $\left(\mathrm{m}^{2}\right) \ldots \ldots \ldots \ldots$

4.3 Total forces on the vehicle $\left(\mathrm{m}^{2}\right) \ldots \ldots \ldots \ldots \ldots$

5.1 Or-design forces for vchicle fusolage $\left(\mathrm{m}^{2}\right) \ldots \ldots \ldots \ldots$

5.2 Forces on Mach 12 vehicle design at $\mathrm{M}=9\left(\mathrm{~m}^{2}\right) \ldots \ldots \ldots \ldots . . \ldots 153$

6.1 Forces on Mach 12 vehicle design at $\mathrm{M}=3\left(\mathrm{~m}^{2}\right), \ldots \ldots \ldots$

7.1 Hypersonic, inviscid arag polar curve fit coefhcients. . . . . . . 177

7.2 Methods used in solving for aerodynamic forces. . . . . . . . 193

7.3 Mach 12 example vehicle properties. . . . . . . . . . 206

7.4 Performance of modified Mach 12 ramjot-powered velicile. . . . 211

B.1 Hydrogen reaction mechanism by Jachimowski [Ref. 61]. . . . . 227 


\section{LIST OF FIGURES}

1.1 The Nonweiler waverider concept [from Ref. 4]. . . . . . . 4

1.2 Example scramjet-powered vehicle geometry [from Ref. 11] . . . . 6

1.3 Example scramjet-powered vehicle geometry [from Ref. 5]. . . . 7

1.4 Example missile geometry [from Ref. 10] . . . . . . . . 7

1.5 Off-design flowfield on caret waverider [from Ref. 14] . . . . . 9 9

1.6 Geometries tested in Ref. 23: a) circular cone waverider, b) elliptic cone waverider, c) elliptic cone with winglets. . . . . . . 10

1.7 Viscous optimized conical waverider [from Ref. 28] . . . . . . 11

1.8 Mach 4 waverider configurations [from Ref. 30]. . . . . . . . 12

1.9 Rocket-powered, osculating cones waverider vehicles investigated by DLR [from Ref. 36] . . . . . . . . . . . . . . 13

1.10 Examples of combined-cycle engine concepts [from Ref.38] . . . 15

1.11 Aerojet's Strutjet engine [from Ref. 43]. . . . . . . . . . 16

1.12 The GTX: an RBCC powered, SSTO vehicle concept currently under development at NASA Glenn Research Center [from Ref. 2]. 16

2.1 Two-view of example vehicle geometry $\left(M_{\infty}=10\right) \ldots \ldots 20$

2.2 Example of waverider compression surface definition using the two-dimensional, inviscid solution for supersonic flow over a wedge. 20 
2.3 Osculating cone waverider development [from Ref. 5], cross-section of exit plane. . . . . . . . . . . . . . . . . . . 23

2.4 Generation of body contour from streamline tracing. . . . . . . 26

2.5 Caret wavericler generated using osculating cones methorl. . . . . 27

2.6 Conical waverider gencrated using osculating cones methorl. . . . 28

2.7 An example of an osculating cones waverider. . . . . . . . . 29

2.8 Volicle inlet geometry. . . . . . . . . . . . . 30

2.9 Vohicle nozzle geometry. . . . . . . . . . . . . . 32

2.10 Characteristic mesh of vehicle nozzle. . . . . . . . 33

2.11 Contours of Mach number on a vehicle nozzle $\left(M_{\mathrm{comb}}=4.13\right) \ldots 34$

2.12 Example volume eloment. . . . . . . . . . . . . 37

2.13 Splitting of volume element in Fig. 2.12 into six tetrahedrons. . . 38

2.14 Surface elements for area and force calculations. . . . . . . . 42

3.1 Vehicle centerline geometry in scramjet mode (not to scale). . . . 46

3.2 Analytical solution of strut/strut interaction $(M=3) \ldots \ldots 47$

3.3 Wave interactions of opposite farnily. . . . . . . . . . . 49

3.4 Wave interactions of same fanily. . . . . . . . . . 50

3.5 Wave/wall interactions. . . . . . . . . . . . . 51

3.6 Computational grid tesed for strut/strut flowfield validation. . . . 52

3.7 Analytical solution to strut/strut interaction superimposed upon pressure contours of CFD Solution $(\mathrm{M}=3) \ldots \ldots \ldots 3$

3.8 Area averaged cxit pressure vs. Mach number for strut/strut interaction.

3.9 Quasi-one-dimensional control volume for species conservation derivation. . . . . . . . . . . . . . . . . . 58 
3.10 Quasi-one-dimensional control volume for energy derivation. . . . 60

3.11 Experimental configuration for Ref $64 \ldots \ldots \ldots \ldots$

3.12 Comparison of non-dimensional wall pressure between experimental results from Ref. 64 and current combustor model. . . . . . . 66

3.13 Experimental configuration for Ref. $66 \ldots \ldots \ldots 68$

3.14 Comparison of non-dimensional wall pressure between experimental results from Ref. 66 and current combustor model. . . . . . . 69

3.15 Sensitivity of mixing profile to perturbation of dependent coefficients. . . . . . . . . . . . . . 70

3.16 Sensitivity of engine model to mixing profile using Billig's engine profile. . . . . . . . . . . . . . . . 71

3.17 Comparison of normal to axial fuel injection profiles. . . . . 73

3.18 RBCC engine scramjet mode geometry, top view (not to scale). . 74

3.19 Mach number and pressure distribution in RBCC engine in scramjet mode. . . . . . . . . . . . . . . 75

3.20 Temperature and mass fraction of $\mathrm{H}_{2} \mathrm{O}, \mathrm{OH}$, and $\mathrm{NO}$ distribution in RBCC engine in scramjet mode. . . . . . . . . . . . 76

3.21 RBCC engine ramjet mode geometry, top view (not to scale). . . 77

3.22 Mach number and pressure distribution in $\mathrm{RBCC}$ engine in ramjet mode. . . . . . . . . . . . . . . . . . 80

3.23 Temperature and mass fraction of $\mathrm{H}_{2} \mathrm{O}, \mathrm{OH}$, and $\mathrm{NO}$ distribution in $\mathrm{RBCC}$ engine in ramjet mode. . . . . . . . . . . . 81

4.1 Profiles of geometries solved for validation. . . . . . . . . 93

4.2 Third derivative of cross-sectional area for original geometry and seventh-order spline addition. . . . . . . . . . . . . . 94 
4.3 Full and close-up view of computational grid for two-dimensional solutions.

4.4 Computational supersonic nondimensionalized pressure distribution over parabolic airfoil $\left(M_{\infty}=1.57\right) \ldots \ldots \ldots 96$

4.5 Computational supersonic nondimensionalized pressure distribution over cubic airfoil $\left(M_{\infty}=1.65\right) \ldots \ldots \ldots 97$

4.6 Supersonic pressure distribution on parabolic airfoil $\left(M_{\infty}=1.57\right)$ and cubic airfoil $\left(M_{\infty}=1.65\right) \ldots \ldots \ldots \ldots$

4.7 Computational subsonic nondimensionalized pressure distribution over full parabolic airfoil $\left(M_{\infty}=0.76\right) \ldots \ldots \ldots 9$

4.8 Computational subsonic nondimensionalized pressure distribution over half parabolic airfoil $\left(M_{\infty}=0.76\right) \ldots \ldots \ldots 100$

4.9 Computational subsonic nondimensionalized pressure distribution over full cubic airfoil $\left(M_{\infty}=0.65\right) \ldots \ldots \ldots \ldots 10 \ldots \ldots$

4.10 Computational subsonic nondimensionalized pressure distribution over half cubic airfoil $\left(M_{\infty}=0.65\right)$.

4.11 Subsonic pressure distribution on parabolic airfoil $\left(M_{\infty}=0.76\right)$ and cubic airfoil $\left(M_{\infty}=0.65\right) \ldots \ldots \ldots \ldots 2 \ldots \ldots \ldots$

4.12 Computational sonic nondimensionalized pressure distribution over full parabolic airfoil.

4.13 Computational sonic nondimensionalized pressure distribution over half parabolic airfoil.

4.14 Computational sonic nondimensionalized pressure distribution over full cubic airfoil 
4.15 Computational sonic nondimensionalized pressure distribution over

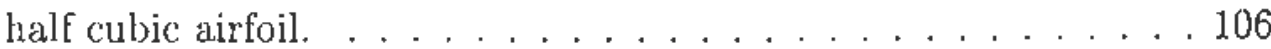

4.16 Sonic pressure distribution on parabolic and cubic airfoit. . . . . . 107

4.17 Full and close-up view of computational grid for axisymmetric solutions. . . . . . . . . . . . . . . . . . . 108

4.18 Supersonic nondimensionalized pressure distribution over parabolic body of revolution $\left(M_{\infty}=1.21\right) \ldots \ldots 109$

4.19 Supersonic pressure distribution over cubic body of revolution $\left(M_{\infty}=1.21\right)$.

4.20 Pressure distribution on parabolic and cubic body of revolution $\left(M_{\infty}=1.21\right)$

4.21 Subsonic nondimensionalized pressure distribution over parabolic body of revolution $\left(M_{\infty}=0.8\right)$.

4.22 Subsonic pressure distribution over cubic body of revolution $\left(M_{\infty}=\right.$ $0.8)$

4.23 Pressure distribution on parabolic and cubic body of revolution $\left(M_{\infty}=0.8\right)$

4.24 Sonic nondinzonsionalized pressure distribution over parabolic body of revolution.

4.25 Sonic nondimensionalized pressure distribution over cubic body

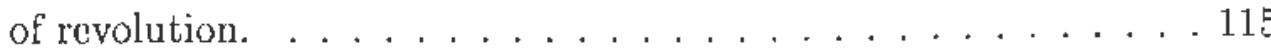

4.26 Pressure distribution on parabolic and cubic: body of rovolution at sonic velocity. ........................ 116

4.27 Bottom surface geometry arıd longitudinal slice used in solving vehicle prossure distribution. 
4.28 Inner hyperbolic grid uscd for computational solutions (including surface grid). . . . . . . . . . . . . . . 121

4.29 Outer box grid (with inner hyperbolic grid) used for computational solutions. . . . . . . . . . . . . . . . . . 122

4.30 Comparison of supersonic CFD results $\left(M_{\infty}=1.65\right) \ldots \ldots 123$

4.31 Comparison of subsonic CFD results $\left(M_{\infty}=0.65\right) . \ldots \ldots 124$

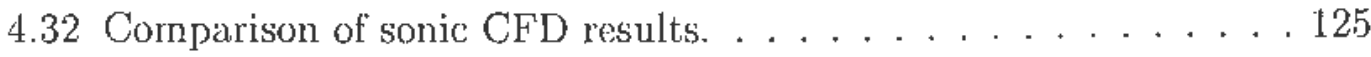

4.33 Side view of nondinensionalized pressure distribution over the

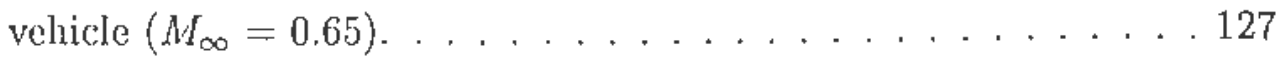

4.34 Pressure coefficient distribution on the lower surface of the vehicle

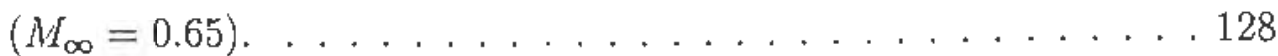

4.35 Normalized pressure distribution on the top surface of the vehicle

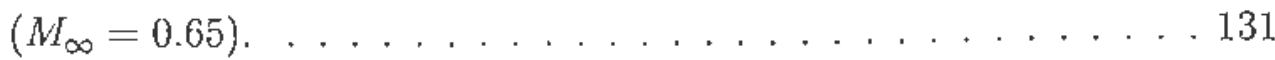

4.36 Side view of nondimensionalized pressure distribution over the vehicle at sonic Mach number. . . . . . . . . . . . . . . 133

4.37 Sonic pressure coefficient distribution on full vehicle. . . . . 134

4.38 Normalized pressure distribution on the top surface of the vehicle at sonic Mach number. . . . . . . . . . . . . . . . 135

4.39 Side view of nondimensionalized pressure distribution over the vehicle $\left(M_{\infty}=1.65\right) \ldots \ldots \ldots \ldots \ldots \ldots \ldots$

4.40 Pressuro coefficient distribution on lower surface of vehicie $\left(M_{\infty}=\right.$

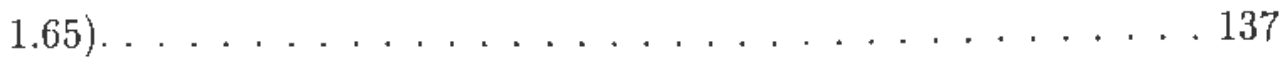

4.11 Pressure coefficient distribution on upper surface of vehicle $\left(M_{\infty}=\right.$

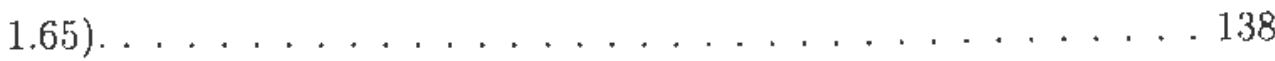


5.1 Current geometry overlayed by Takashima's geometry, planform $\left(M_{\infty}=10, \alpha=0\right)$

5.2 Curront geometry overlayed by Takashima's geometry, including GASP solution for pressure contours, cross-section $\left(M_{\infty}=10, \alpha\right.$

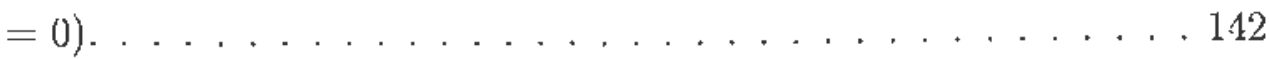

5.3 Inviscid L/D as a function of angle of attack $\left(M_{\infty}=10\right) \ldots \ldots 143$

5.4 Inviscid $\mathrm{L} / \mathrm{D}$ as a function of Mach number $(\alpha=0) . \ldots \ldots 145$

5.5 Normalized pressure distribution over Mach 12 vehicle fuselage at. on-design conditions. . . . . . . . . . . . . . 147

5.6 Normalized pressume distribution over lower surface of Mach 12 vehicle fuselage at on-design conditions. . . . . . . . . 148

5.7 Normalized pressure distribution over upper surface of Mach 12 vehicle fuselage at on-design conditions. . . . . . . . . 150

5.8 Normalized pressure distribution over Mach 12 vehicle fuselage

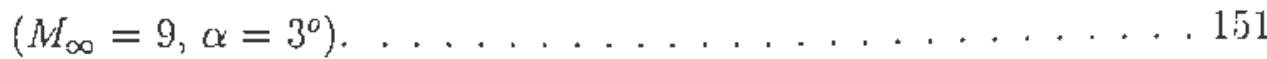

5.9 Normalized pressure distribution over lower surface of Mach 12 velicle fuselage $\left(M_{\infty}=9, \alpha=3^{\circ}\right) \ldots \ldots \ldots \ldots 2$

5.10 Normalized pressure distribution over upper surface of Mach 12 vehicle fuselage $\left(M_{\infty}=9, \alpha=3^{\circ}\right)$.

5.11 Normalized pressure distribution over Mach 12 vehicle fuselage $\left(M_{\infty}=9, \alpha=-3^{\circ}\right)$.

5.12 Normalized pressure distribution over lower surface of Mach 12 vehicle fuselage $\left(M_{\infty}=9, \alpha=-3^{o}\right)$.

5.13 Normalized pressure distribution over upper surface of Mach 12 vehiclo fusclage $\left(M_{\infty}=9, \alpha=-3^{\circ}\right) \ldots \ldots \ldots \ldots \ldots$ 
5.14 Inlet lip geometry. . . . . . . . . . . . . . 158

6.1 Nomalized presstre distribution over Mach 12 vehicle fusclage $\left(M_{\infty}=3, \alpha=3^{n}\right)$.

6.2 Normalized pressure distribution over lower surface of Mach 12 vehicle fuselage $\left(M_{\infty}=3, \alpha=3^{\circ}\right)$.

6.3 Normalized pressure distribution over upper surface of Mach 12 velicle fusclage $\left(M_{\infty}=3, \alpha=3^{\omega}\right)$.

6.4 Normalized pressure distribution over Mach 12 vehicle fuselage $\left(M_{\infty}=3, \alpha=-3^{\circ}\right)$

6.5 Normalized prossure distribution over lower surface of Mach 12 vehicle fuselage $\left(M_{\infty}=3, \alpha=-3^{\circ}\right) \ldots \ldots \ldots \ldots \ldots$

6.6 Normalized pressure distribution over upper surface of Mach 12 vehicle fusclage $\left(M_{\infty}=3, \alpha=-3^{o}\right)$.

7.1 Hypersonic lift $\left(\mathrm{m}^{2}\right)$ of Mach 12 vohicle as a function of Mach number.

7.2 Hypersonic lift $\left(\mathrm{m}^{2}\right)$ of Mach 12 vehicle as a function of angle of attack. . . . . . . . . . . . . . . . . . 172

7.3 Hypersonic lift coefficient of Mach 12 vehicle as a function of angle of attack.

7.4 Hypersonic, inviscid drag $\left(\mathrm{m}^{2}\right)$ of Mach 12 vehicle as a function of Mach number. . . . . . . . . . . . . . . . . . . 174

7.5 Hypersonic, inviscid $\mathrm{drag}\left(\mathrm{m}^{2}\right)$ of Mach 12 vehicle as a function of angle of attack. . . . . . . . . . . . 175 
7.6 Hypersonic, inviscid drag coefficient of Mach 12 vehicle as a function of angle of attack. . . . . . . . . . . . . . 176

7.7 Hypersonic, inviscid drag polar of Mach 12 vehicle $\ldots \ldots . . .176$

7.8 Comparison of curve fits to Newtonian and tangent-wedge flow. . 178

7.9 Supersonic lift $\left(\mathrm{m}^{2}\right)$ of Mach 12 vehicle as a function of Mach number. . . . . . . . . . . . . . . . . . . 180

7.10 Supersonic lift $\left(\mathrm{m}^{2}\right)$ of Mach 12 vehicle as a function of angle of attack.

7.11 Supersonic lift coefficient of Mach 12 vehicle as a function of angle of attack.

7.12 Supersonic, inviscid drag $\left(\mathrm{m}^{2}\right)$ of Mach 12 vehicle as a function of Mach number. . . . . . . . . . . . . . . . . . 183

7.13 Supersonic, inviscid drag $\left(\mathrm{m}^{2}\right)$ of Mach 12 vchicle as a function of angle of attack. . . . . . . . . . . . . . . . 184

7.I4 Supersonic, inviscid drag coefficient of Mach 12 vehicle as a function of angle of attack. . . . . . . . . . . . . . 185

7.15 Supersonic, inviscid drag polar of Mach 12 vehicle. . . . . . . 185

7.16 Transonic lift $\left(\mathrm{m}^{2}\right)$ of Mach 12 vehicle as a function of Mach number. 187

7.17 Transonic lift $\left(\mathrm{m}^{2}\right)$ of Mach 12 vehicle as a function of angle of attack.

7.18 Transonic lift coefficient of Mach 12 vehicle as a function of angle

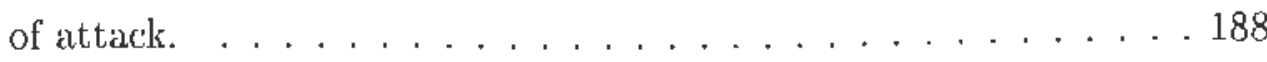

7.19 Transonic inviscid drag $\left(\mathrm{m}^{2}\right)$ of Mach 12 vehicle as a function of Mach number. . . . . . . . . . . . . . . . . . . . 189 
7.20 Transonic, inviscid drag $\left(\mathrm{m}^{2}\right)$ of Mach 12 vehicle as a function of angle of attack. . . . . . . . . . . . . . 190

7.21 Transonic, inviscid drag coefficient of Mach 12 vehicle as a function of angle of attack. . . . . . . . . . . . . 191

7.22 Transonic, inviscid drag polar of Mach 12 vehicle. . . . . . . . 192

7.23 Lift on example Mach 12 vehicle for full Mach number range. . . . 195

7.24 Percent error in lift on example Mach 12 vehicle for full Mach number range. . . . . . . . . . . . . . . . . . . . .

7.25 Error in magnitude of lift on example Mach 12 vehicle for full Mach number range. . . . . . . . . . . . . . 196

7.26 Lift coefficient on example Mach 12 vehicle for full Mach number range. . . . . . . . . . . . . . . . . . 197

7.27 Pressure drag on example Mach 12 vehicle for full Mach number range. . . . . . . . . . . . . . . . . 198

7.28 Percent crror in pressure drag on example Mach 12 vehicle for full Mach number range. . . . . . . . . . . . . . . . 199

7.29 Inviscid drag coeficient on example Mach 12 vehicle for full Mach number range. . . . . . . . . . . . . . . . . 200

7.30 Transonic, viscous airframe drag coefficient as a function of Mach number. . . . . . . . . . . . . . . . . . 201

7.31 Inviscid and viscous airframe $L / D$ as a function of Mach number. 203

7.32 Inviscid and viscous airframe $L / D$ as a function of angle of attack. 205

7.33 Three-view of Mach 12 scranjet-powered example vehicle. . . . 205

7.34 L/D vs. angle of attack for example Mach 12 vehicle $(\phi=1), \ldots 207$

$7.35 I_{\text {si }_{\mathrm{e} f f}}$ vs. angle of attack for cxample Mach 12 vehicle $(\phi=1) . \quad .209$ 
7.36 Throe-view of modified Mach 12 ramjet-powered vehicle. . . . . 210

7.37 Trajectory and velocity profile as a function of range and percent fuel remaining. . . . . . . . . . . . . . . . . . . . . . . .

7.38 Mach number and angle of attack profile as a function of range and percent fuel remaining. . . . . . . . . . . . . 214 


\section{LIST OF SYMBOLS}

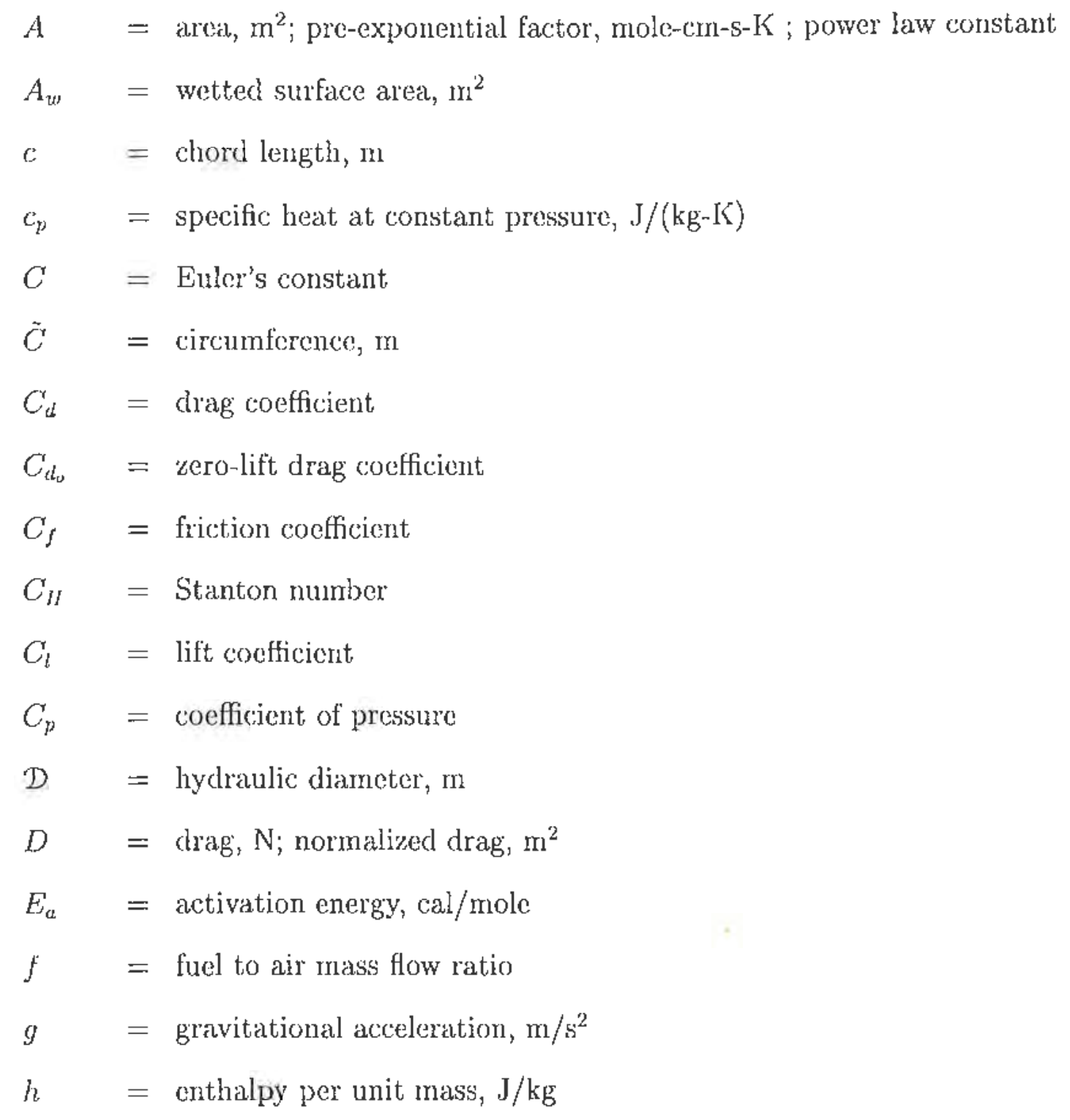




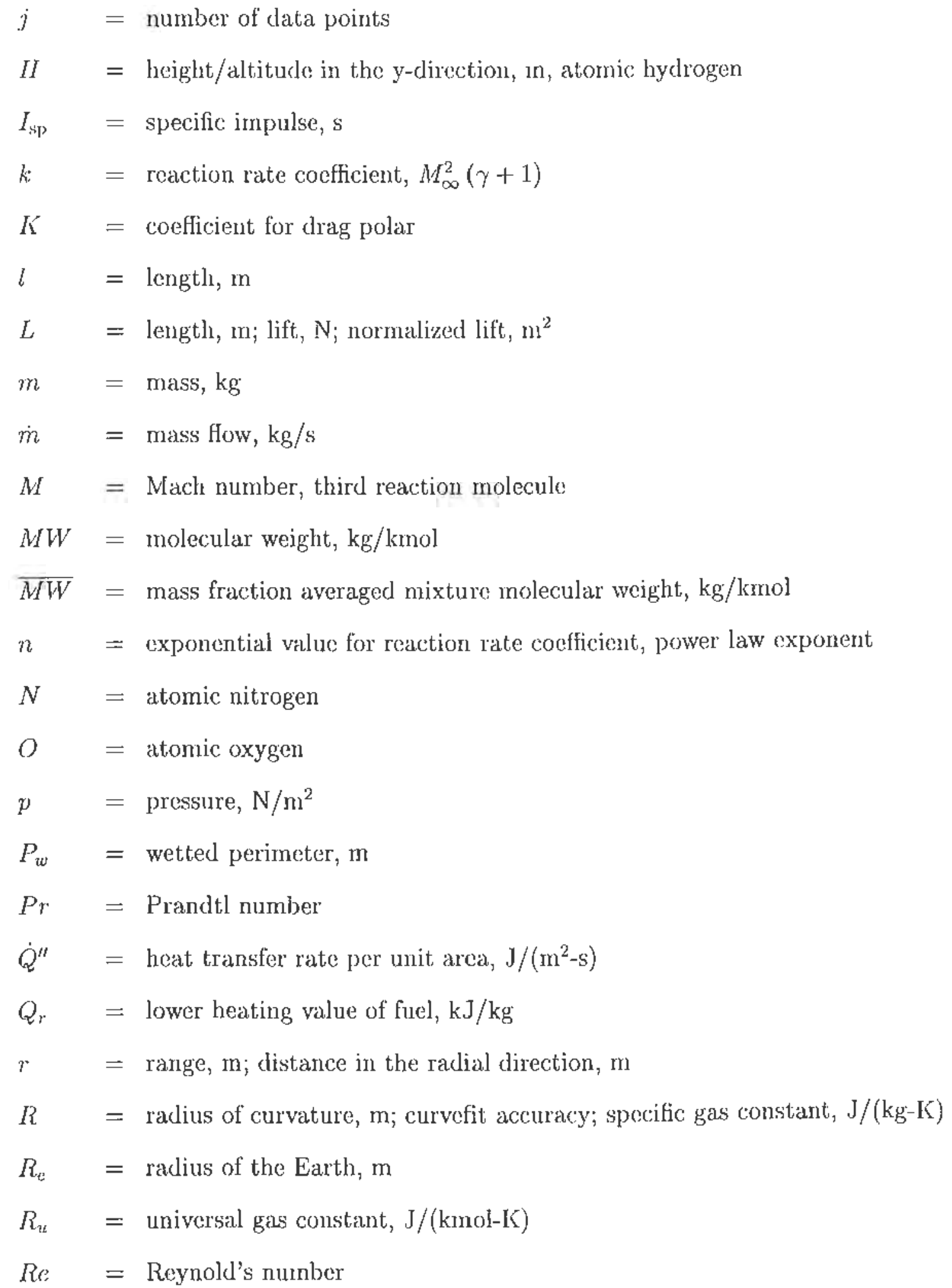




$$
\begin{aligned}
& S=\text { cross-sectional area, } \mathrm{m}^{2} \\
& T=\text { temperature, } \mathrm{K} \text {; thrust, } \mathrm{N} \\
& T h=\text { thrust, } \mathrm{N} \\
& u=\text { perturbation velocity, } \mathrm{m} / \mathrm{s} \\
& \bar{u} \quad=\text { nondimensionalized perturbation velocity } \\
& U \quad=\text { velocity, } \mathrm{m} / \mathrm{s} \\
& V \quad=\text { velocity, } \mathrm{m} / \mathrm{s} \\
& \bar{V} \quad=\text { nondimensionalized velocity } \\
& \text { Vol }=\text { volume, } \mathrm{m}^{3} \\
& x, y, z=\text { coordinates in Cartesian coordinates, } \mathrm{m} \\
& Y \quad=\text { mass fraction } \\
& Z \quad=\text { ordinate of geometry, } \mathrm{m} \\
& \alpha \quad=\text { angle of attack } \\
& \beta \quad=\text { shock wave angle } \\
& \gamma \quad=\text { ratio of specific heats } \\
& \gamma_{f} \quad=\text { flight path angle } \\
& \delta=\text { wedge angle of waverider forebody } \\
& \epsilon \quad=\text { ratio of fuel velocity to freestream velocity } \\
& \eta \quad=\text { efficiency } \\
& \theta \quad=\text { angle from axis of cone to a ray of solution } \\
& \bar{\theta}=\text { boundary layer momentum thickness, } \mathrm{m} \\
& \mu \quad=\text { viscosity coefficient, } \mathrm{kg} /(\mathrm{m}-\mathrm{s}) \\
& \xi \quad=\text { dummy variable for integration, } m \\
& \pi \quad=\text { volume fraction } \\
& \rho \quad=\text { density, } \mathrm{kg} / \mathrm{m}^{3}
\end{aligned}
$$




$$
\begin{array}{ll}
\tau & =\text { shear stress, } \mathrm{N} / \mathrm{m}^{2} ; \text { thickness ratio } \\
\phi & =\text { equivalence ratio; velocity potential } \\
\dot{\omega} & =\text { molar production rate, } \mathrm{kmol} /\left(\mathrm{s}-\mathrm{m}^{3}\right)
\end{array}
$$

Subscripts

$$
\begin{aligned}
& 2 \mathrm{D}=\text { two-dimensional solution } \\
& \text { added }=\text { species added to engine flowfield } \\
& \text { avg }=\text { average } \\
& \text { aw }=\text { adiabatic wall } \\
& \text { axi }=\text { axisymmetric solution } \\
& \text { bot }=\text { amount on the bottom } \\
& c=\text { cone } \\
& \text { cancel }=\text { shock cancellation location } \\
& \text { cent }=\text { centrifugal } \\
& \text { comb }=\text { combustor } \\
& \text { cowl }=\text { cowl location } \\
& \text { cs } \quad=\text { cross-section } \\
& e \quad=\text { edge of boundary layer; elliptic; end of isolator } \\
& \text { eff }=\text { effective } \\
& \text { end }=\text { end location } \\
& \text { eng }=\text { engine } \\
& f \quad=\text { forebody; fuel } \\
& h \quad=\text { hyperbolic } \\
& \mathrm{H}_{2} \quad=\text { liquid hydrogen } \\
& i \quad=\text { denotes species } i \text {; incompressible; inlet of isolator }
\end{aligned}
$$




$$
\begin{aligned}
& \text { inc }=\text { incremental value } \\
& \text { isolator }=\text { isolator } \\
& \text { loc }=\text { local value } \\
& \text { max }=\text { maximum } \\
& \text { min }=\text { minimum } \\
& \text { mix }=\text { fuel mixing } \\
& \text { o } \quad=\text { total or stagnation conditions } \\
& O_{2}=\text { liquid oxygen } \\
& p \\
& \text { plan }=\text { planform } \\
& \text { quad }=\text { quadratic interpolation } \\
& r \\
& \text { sp } \quad=\text { radial direction; portion available for reaction } \\
& \text { st } \quad=\text { stoichiometric conditions } \\
& \text { start }=\text { start location } \\
& \text { stream }=\text { streamwise direction } \\
& t \\
& \text { tc }
\end{aligned}
$$


$z \quad=$ derivative with respect to $z$

$\theta \quad=$ direction norma! to radial direction

$\overline{0} \quad=$ based on boundary layer momentum thickncss

$\infty=$ freostrean conditions

Superscripts

* cvaluated at reference temperaturo

, $\quad$ differentiation 


\section{Chapter 1}

\section{Introduction}

\subsection{Motivation}

The Nationa Aeronantics and Space Administration (NASA) recently announced the Space Launch Initiative (SLI). The SLI was developed as a result of an industry-led study to determine the direction NASA should take to dovelop the next two generations of reusable launch vehicles (RLVs). These RLVs would eventually replace the space shuttie (otherwise known as the first generation of R.LV) as the premier launch vehicles in the NASA fleet. The primary impetus for replacing the space shuttle is the reduction of launch costs (currontly at approximately $\$ 10,000$ per pound of payload) and launch failures (currently under $1 \%$ ). Under the SLI requirentents, the sccond generation of launcil vehicles will have launch costs on the order of $\$ 1,000$ per pound of payload and a launch failure probability of under $0.01 \%$. For the third generation of launch velicles, the SLI requircments will have launch costs reduced to $\$ 100$ per pound of payload with launch failure probability under $0.0001 \%$ (or one in à million).

The primary focus for the second generation of RLV is on rocket-based propulsion systems. These engines have a long history of development, experimont, and flight. Cost reduction for these vehicles is achicved by increasing 
engine efficiency, reducing weight, reducing turn-around time (the time between successive launches), reducing ground personnel necessary for launch, as well as other aspects. A major drawback of rocket systems is the necessity to carry oxidizer on board to burn the vehicle's fuel. The increase in weight from the oxidizer must be compensated by more engine thrust, thus necessitating more fuel. A second drawback of rocket systems is that, through the gradual improvements in efficiency from technological advances, the upper limits in engine efficiency will soon be reached. An example is the space shuttle main engine (SSME) which has a specific impulse of approximately 455 seconds. The theoretical limit for a hydrogen-oxygen fueled rocket engine is approximately 526 seconds. Thus, the space shuttle main engine is currently running at approximately $87 \%$ of the theoretical maximum. Even if advances are made to improve the efficiency of the SSME to the theoretical maximum, the performance of these engines will not dramatically reduce launch costs to the one hundred dollar level envisioned by NASA for the third generation of RLV.

Barring the use of a radical engine system such as a nuclear rocket, a way to increase engine efficiency is by using an air-breathing engine system. These systems (such as jet engines) use the oxygen in the atmosphere as an oxidizer, thus eliminating the need to carry oxidizer on board. To use atmospheric oxygen, the vehicle must stay within the atmosphere longer than rocket systems to accelerate to orbital velocities. Air-breathing vehicles may take advantage of the increased time in the atmosphere by generating aerodynamic lift from their wings and/or fuselage (as opposed to rockets which generate nearly all of their lift from their engines). Aerodynamic lift also acts to increase the efficiency of air-breathing vehicles compared to rocket vehicles, but can be countered by aerodynamic drag if the vehicle is not properly designed.

Considering the benefits discussed above, it is no surprise that for the third generation of RLV, NASA is investigating vehicles which use air-breathing en- 
gines 1,2. One major engine system being considered for the third generation of RLV is the rocket-based combined-cycle (RBCC) engine. This engine system is essentially a low-weight compromise between producing high thrust at low flight speeds (from a rocket engine) and producing efficient thrust at high speeds (using a ramjet and/or a scramjet engine). At low speeds, rockets are used to propel tho vohicle. At higher speeds (Mach 1 to 2), the engine transitions from a rocket to a ramjet engine. As the vehicle continues to accolerate past Mach 6 to 8 , the ranjet transitions to a supersonic combustion ramjet (scramjet). Finally, as the vohicle nears the edge of the atmosphere (where oxygen levels are small), the engine transitions back to a rocket to insert tho vehicle into orbit.

In the current work, an RBCC engine concept will be incorporated into a waverider-based engine-airframe integrated vehicle as a proposed third generation RLV. In the following section, a brief history of waveriders, waverider-based enginc-airframe integrated vehicles, off-design behavior of waveriders, and RBCC engines will be provided. The review of the previous work will serve as a guide for demonstrating tho contributions that the current work will make to the ficld.

\subsection{Previous Work}

\subsubsection{Waverider Geometries}

The vehicle geometry currently under investigation is based around a hypersonic waverider forebody. The waverider concept was initially proposed by Nonweiler: as an example of a high-speed re-entry geometry that would have high lift coefficient. The reasoning behind Nonweiler's work was based on lowering peak heating rates on re-entry vehicles for manned space launch. By lowering wing loading, an aerodynamic lifting re-entry geometry would have substantially lower surface temperatures than a ballistic re-entry geornetry. Nonweiler's so- 


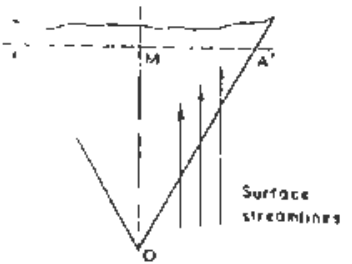

(a) Single Slrock

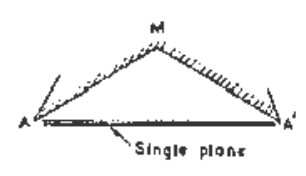

Figure 1.1: The Nonweiler waverider concept [from Ref. 4]

Iution to this problem was a fying wing design with a delta planform, so that the wing loading was dispersed over the entire fuselage. Ile selected supersonic wedge flow to model the lower surface of the geometry and suggested that this geometry "may serve as a useful basis for a perturbation" a An example of the Nonweiler geometry (also called the "caret" waverider because of its rescmblance to the typographical symbol " $\Lambda$ ") is shown in Fig. 1.1 from Nonweiler.4.

In principle, a waverider may be defined as any shape that has inviscid bow shock wave attachment at its sharp loading odges. As a result of shock wave attachment, the high pressure flowfield generated from the waverider comprossion surface is contained underneath the vehicle. Pressure containment minimizes losses due to pressure spillage and gives waverider slapes high lift to drag ratio $L / D$ at high lift. cocflicient.

The entire flowfield between the waverider compression surface and the bow shock is known due to the inverse nature of waverider design. Knowledge of the compression flowficld of these geometries makes them very usoful in the design of engine-airframe integrated vehicles, where accurate knowledge of cingine inlet conditions is imperative. Although Nonweiler proposed a wodge-derived waveridor gcometry, a waverider need not be generated from only suporsonic wedge flow. A supersonic flowfield solution over any arbitrary body may be used 
as a basis for generating a waverider shape. Thus, the Rexibility of geometry definition allows for rapid vehicle design and optimization.

The waverider concept has several inherent advantages over a generic, highspecd, lifting body. The first advantage is that the entire inviscid flowficld located under the compression surface is known from the flowficld solution used to gencrate the waverider. Innowledge of the flow exiting the waverider aids in predicting the acrodynamics on the fuselage of the vehicle as well as the flow properties entcring into the combustor. Second, at the on-design condition, the waverider lias full shock wave attachment, thereby preventing pressure leakage. Thus, any flow that is compressed by the waverider goes directly into production of lift or into the engine, resulting in higher aerodynamic efficiency. Compression of the flow prior to entering the engine reduces the compression requirements that the engine must meet for the proper fuel ignition conditions. Third, the gencration and analysis of waverider geometries is a simple task, resulting in complex vehicle geometries with known aerodynamic properties that may be quickly solved on a computer.

There is a long history of waverider dovelopment, experimentation, and validation. Takashima ${ }^{5}$ discusses the history of waverielers in more detail, and suggests that the reader consult Eggers et al. ", Kuchcmann ", Rasmussen ${ }^{8}$, or Roe $^{g}$ for fluther insight into the concept of the waverider. Hand-in-hand with waverider development has becn wavericler optimization. Takashinda ${ }^{5}$ and Starkcy ${ }^{10}$ both discuss in detail the history of waverider optimization and the "state of the art" in developing optimal waverider geometries.

\subsubsection{Waverider-Based Engine-Airframe Integration}

An extensive amount of rescarch has been performed at the University of Maryland within the last few years on integrating high-speed engines with 


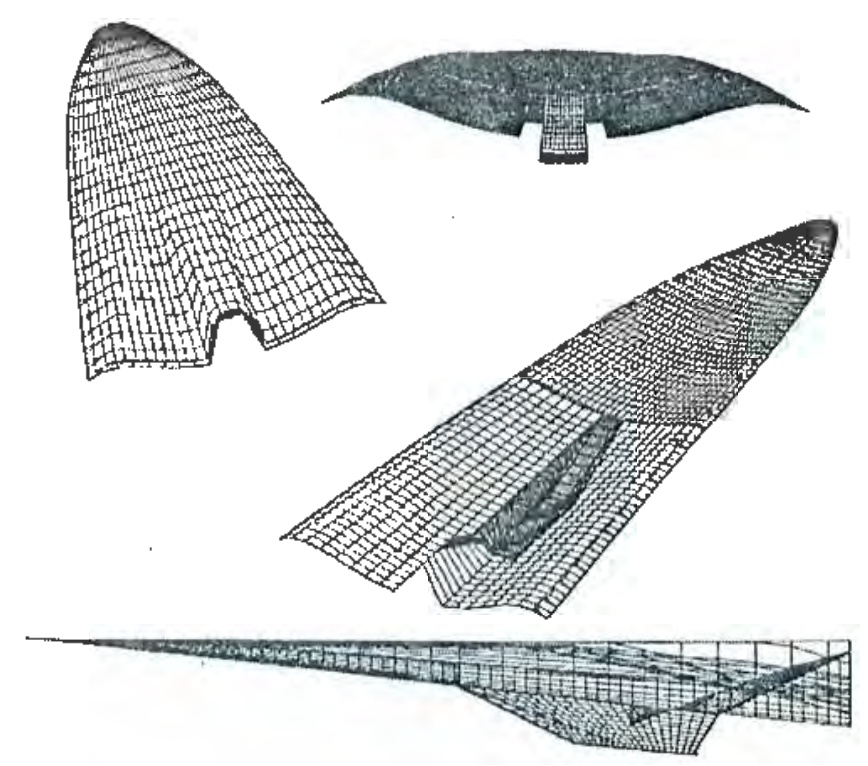

Figure 1.2: Example scramjet-powered vehicle geometry [from Ref. 11].

waverider-based vehicles. One of the first works to incorporate a scramjet model with a conical waverider-based vehicle was performed by $O^{\prime} N$ eill ${ }^{11}$. An cxample geomctry from Ref. 11 is shown in Fig. 1.2. These vehicles were powered by lydrogen-fueled scramjet engines, and optimizations were performed for both cruising and accelerating missions.

Takashima ${ }^{5}$ incorporated a hydrogen-fueled scramjet engine with an osculating cones waveriderbased vehicle. An examplo geometry from Ref. 5 is shlown in Fig. 1.3. The vehicle geometries developed by Takashima are the basis for the vehicle geometries used in the current work. Takashima optimized these vehicles for cruising conditions and investigated the hypersonic off-design bohavior of osculating cones waverider forebodies.

Starkey ${ }^{10}$ incorporated a hydrocarbon-fueled scramjet engine with a wodgederived waverider-based vehicle as an application for a hypersonic missile design. An example geometry from Ref. 10 is shown in Fig. 1.4. These geometries were optimized using genetic algorithms for eruising conditions, where the primary 


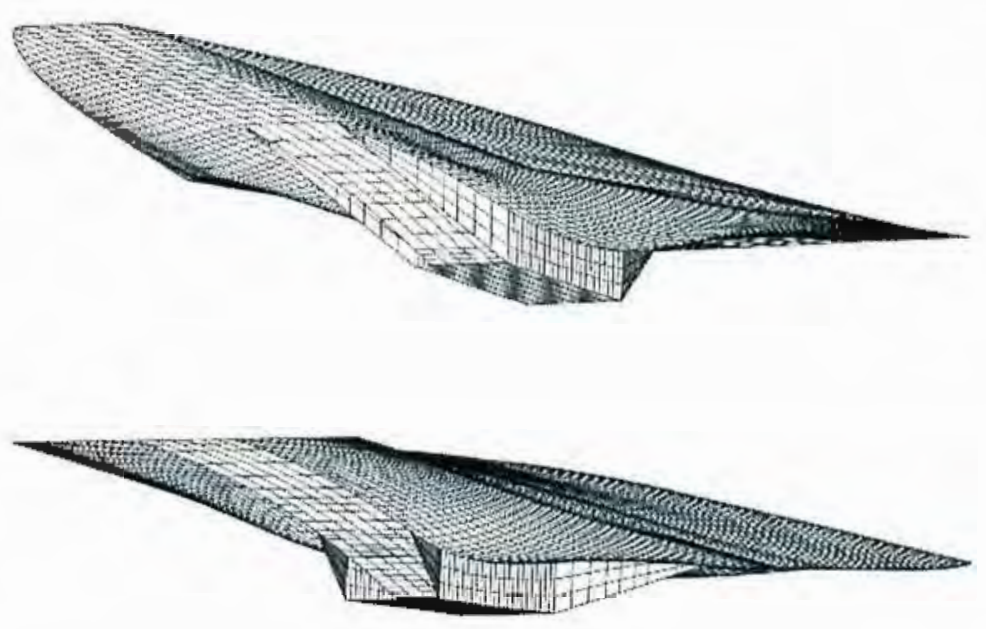

Figure 1.3: Example scramjet-powered vehicle geometry [from Ref. 5].
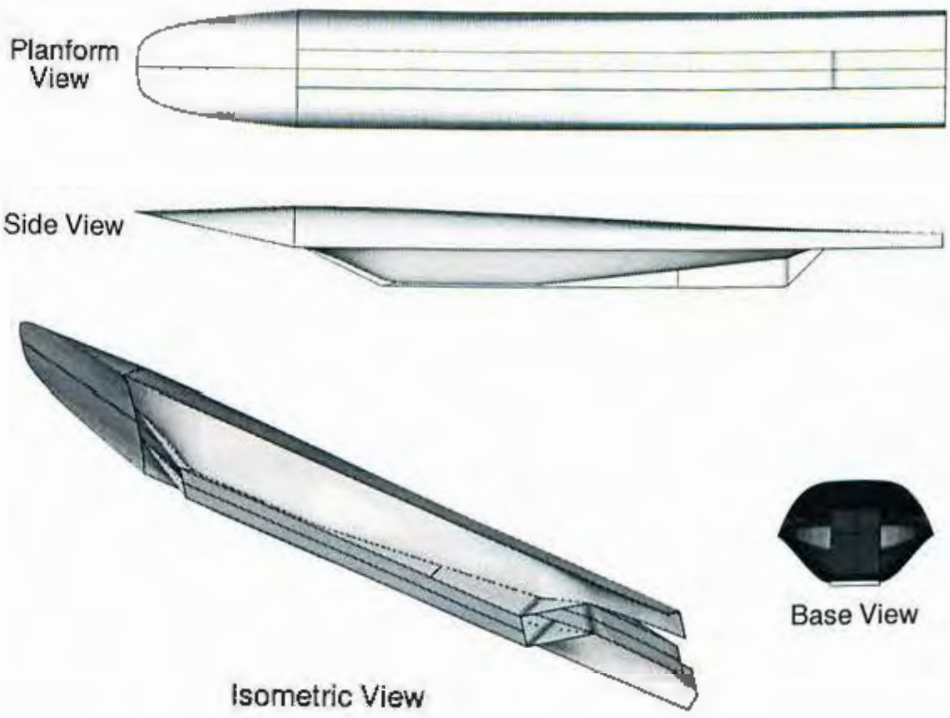

Figure 1.4: Example missile geometry [from Ref. 10]. 
focus was developing an inlet and engine design that would allow a hydrocarbon fuel to mix and burn within the length-constrained combustor.

The history of engine-airframe integration has been reviewed by O'Neill ${ }^{11}$, Takashima ${ }^{5}$, and Starkey ${ }^{10}$. The reader is referred to these dissertations for more details into previous work performed on engine-airframe integration issues.

\subsubsection{Off-Design Waverider Performance}

Considering the methodology behind waverider surface generation, there can be only one Mach number and angle of attack that correspond to "on-design" conditions. Hence, for all practical purposes, a waverider-based engine-airframe integrated vehicle will most likely always be flying at non-optimal or "off-design" conditions. In fact, this has been one of the major criticisms against using waverider geometries on high-speed vehicle designs. An interesting note is that in Nonweiler's initial paper that proposed the caret waverider, he notes that "quite plainly the very unusual shape [waverider] may well have unusual flying qualities at low speeds!" 3.

Long points out several other characteristics of waverider geometries that are critical to address before application to actual vehicle designs: "aeropropulsion integration, aerothermal heating, stability and control, nonconical or nonplanar bow shocks, shock-wave impingement, leading-edge vortices, degradation of theoretical performance due to viscous effects, effect of leading-edge radii, upper surfaces not aligned with freestream, and shock thickening due to Knudsen number effects" ${ }^{12}$. All of the above quoted effects may be considered to be "off-design effects". Aeropropulsion integration has been extensively investigated in recent years with the works of O'Neill ${ }^{11}$, Takashima ${ }^{5}$, and Starkey ${ }^{10}$, as discussed above. Aerothermal heating has been investigated by Vanmol ${ }^{13}$. Stability and control have been investigated by Tarpley ${ }^{14}$ and Rudd ${ }^{15}$. Nonplanar 


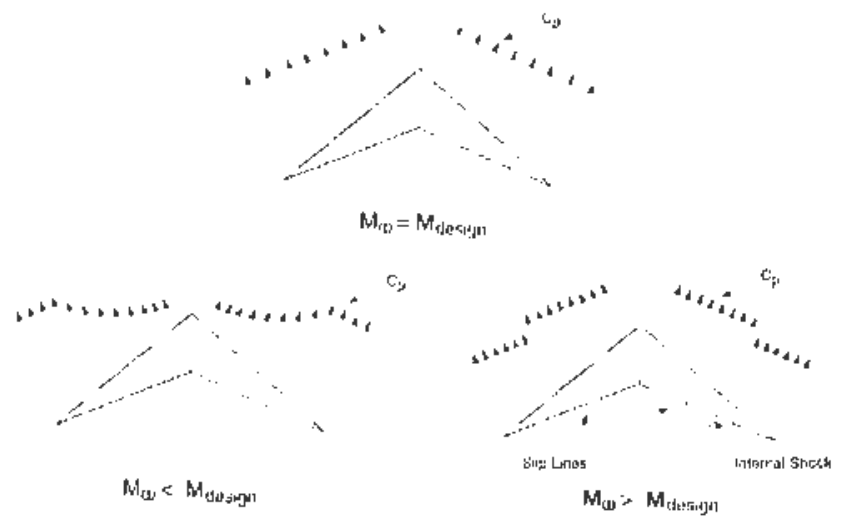

Figure 1.5: Off-design flowfield on caret waverider [from Ref. 14].

bow shocks have been investigated by Bowcutt ${ }^{16}$, O'Neill " Takashima ${ }^{5}$, and Starkey ${ }^{10}$. Viscosity and its effects on waverider optinnization has been investigated by Bowcutt ${ }^{16}$. Leading edge effects have been investigated by Kothari et al. ${ }^{17}$, Vanmol ${ }^{13}$, O'Brien ${ }^{18}$, and $O^{\prime} B r i c n$ et al. ${ }^{19}$.

A few years after Nonweiler's initial conception of the waverider, off-design studies of the flowfield underneath the caret waverider were undertaken. The first. study to discuss the off-design shock structure for the geometry was Venn and Flower ${ }^{20}$. From a theoretical basis, Venn and Flower develop the methodology of predicting the shock shape under a caret waverider for off-design Mach number and angle of attack. The general shock strueture under the caret waverider is shown in Fig. 1.5 from Ref. 14. An estimation of the alerodynamic performance of off-design caret waveriders was shown by Squire ${ }^{21}$ to be kccurately predicted using thin-shock-layer theory. Squire also compares waveriders to flat wings and incorporates real gas effects into the analysis. Townend ${ }^{22}$ discusses the application of a caret waverider geometry to a space shuttle class vehicle, and reviews the geometry's aerodynamic performance across the full Mach number range to and from orbit.

After the mid 1970's, the focus of waverider work shifted toward geometries 

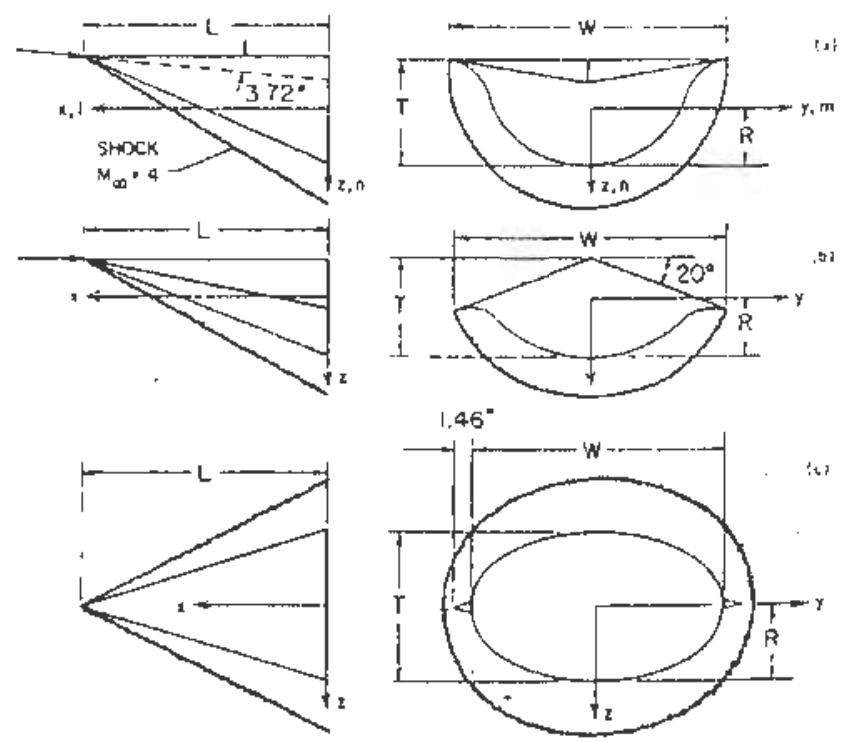

Figure 1.6: Geometries tested in Ref. 23: a) circular cone waverider, b) clliptic cone waverider, c) elliptic cone with winglets.

based on axisymmetric or non-wedge based flowfields. Early off-design work on these type of geometrics tonded toward wind tunnel tosting. Rasmussen et al. ${ }^{23}$ were some of the first to test the near off-design behavior of cone-derivod waveriders (tho shapes tested are shown in Fig. 1.6 from Ref. 23), showing that the near-design performance of waveriders is not significantly degraded from ondesign predictions. Vanhoy ${ }^{24}$ was the first to test the low-speed performance of viscous optimized conical waveriders generated by Corda ${ }^{25}$, and found that the gcometries may be modeled quite accurately as delta planform wings with camber. Gillum ${ }^{25}$ investigated the effects of leading edge blunting on a Mach 14 viscous optimized waverider (generated using the Maryland Axisymmetric Wavericle: Program MAXWAR,P ${ }^{27}$ ) for a range of Mach numbers between 10 and 16.5 and a range of angles of attack between $-10^{\circ}$ and $25^{\circ}$. The results from Gillum's experiments showed that the drag polar of these geonctries hat exponents of 1.75 (by concidence, the averaged result between Newtonian flow 

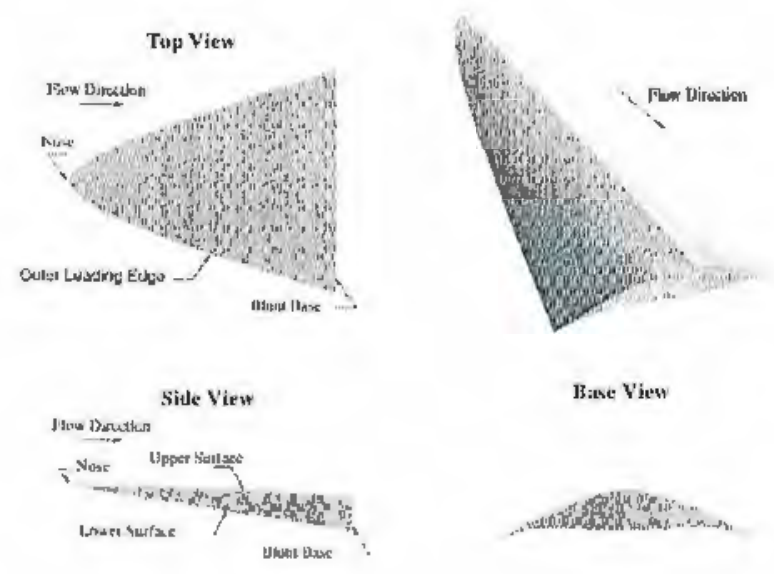

Figure 1.7: Viscous optimized conical waverider [from Ref. 28].

and tangent-wedge flow).

With the advent of cheaper and more powerful computers, computational studies of the off-design performance of waverider geometries became more prevalent in the late 1980's. Long ${ }^{12}$ was one of the first to perform computational off-design studies of inviscid waverider geometries, using waveriders generated by the Rasmussen technique. A Mach 4 designed waverider was solved over a range of Mach numbers between 0.6 and 15 and (at Mach 4) a range of angles of attack between $-10^{\circ}$ and $15^{\circ}$. Cockrell ${ }^{28}$ investigated viscous optimized conical waveriders designed at Mach 4 for off-design Mach numbers between 3.5 and 4.5 and angles of attack between $0^{\circ}$ and $10^{\circ}$. One of the geometries solved by Cockrell is shown in Fig. 1.7 from Ref. 28. He et al. ${ }^{29}$ performed inviscid and viscous off-design computations on a collection of 8 Rasmussen-type waveriders and investigated the effects of leading edge blunting on the aerodynamic performance of the geometries. All of the above studies showed that the near-design performance of waveriders was not significantly degraded from their on-design predictions.

The next logical step in the study of off-design behavior of a waverider vehicle 


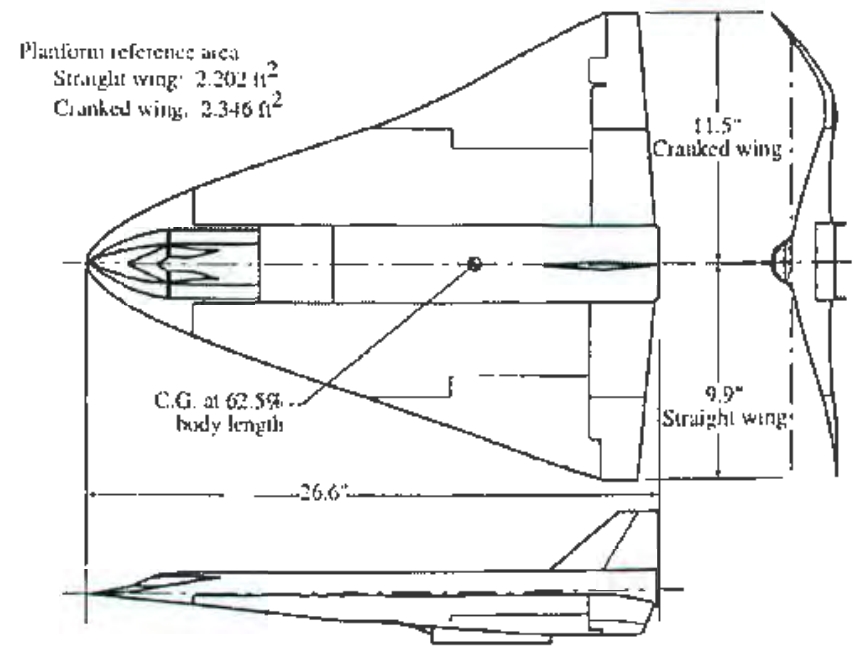

Figure 1.8: Mach 4 waverider configurations [from Ref. 30].

is to add integration effects such as pilot canopies, engine components, control surfaces, and base closure. A series of experimental and computational studies were performed on a Mach 4 viscous optimized waverider configuration shown in Fig. 1.8 from Pegg et al. ${ }^{30}$ Cockrell et al. ${ }^{31}$ performed experimental and computational investigations of the geometry in Fig. 1.8 for a range of Mach numbers between 1.6 and 4.63 and a range of angles of attack between $-8^{\circ}$ and $12^{\circ}$. Detailed component effects on vehicle integration were investigated for the lift, drag, and moment on the vehicle. Pegg et al. ${ }^{30}$ performed low speed wind tunnel testing of the geometry in Fig. 1.8 and showed the effects of control surfaces on the aerodynamic performance, stabiitty, and control of the vehicle.

Recently, the osculating cones waverider geometry (proposed by Sobieczky ct al. ${ }^{2}$ ) has received attention for its chlaracteristics at off-design. Subsonic wind tunnel testing was performed by Miller of al. ${ }^{33}$ and found that osculating cones waveriders perform in a similar fashion as conical waverider geometries. Takashima ${ }^{5}$ investigated the off-design performance of these geometries for offdesign Mach numbers at zero angle of attack and off-design angles of attack at 

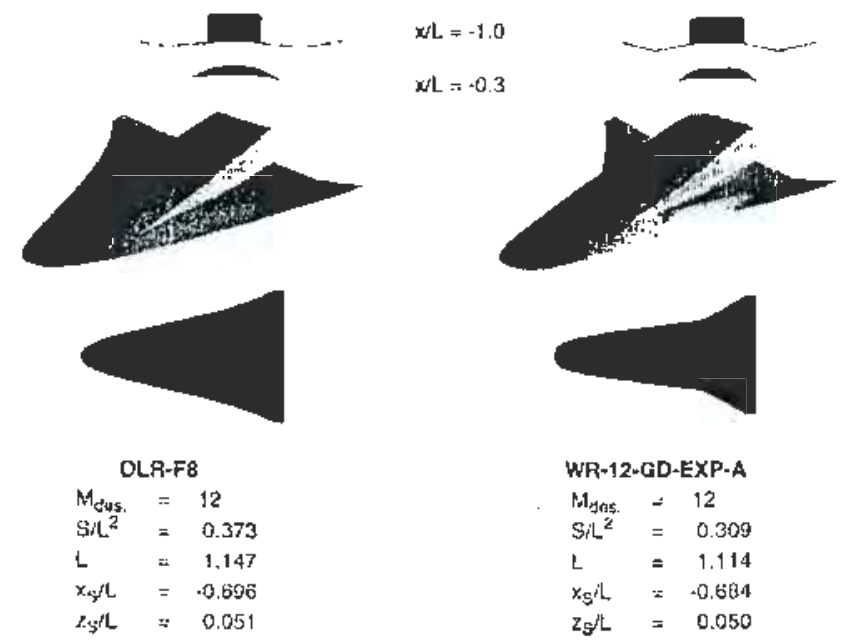

Figure 1.9: Rocket-powered, osculating cones waverider vohicles investigated by DLR [from Ref. 36].

design Mach number, and used computational solutions to validate an analytical method for aerodynamic prediction reported by Grantz ${ }^{3.1}$. Germany's DLR ${ }^{36}$ has thoroughly investigated the off-design performance of gothic planform osculating cones waverider geometries (sce Fig. 1.9 from Strohmeyer ${ }^{36}$ ) for application to rocket-powered vehicles.

Finally, Starkey et al. ${ }^{37}$ developed an analytic:al method for predicting the off-design behavior of wedge-derived waverider geometries for Mach numbers between 5 and 15 and for angles of attack between $-6^{\circ}$ and $6^{\circ}$. Other than the works of Takashimas and Starkey ${ }^{37}$ (and to a limited extent, the works of Squire21 and Tarpley ${ }^{14}$ ), there has been little work done on analytically predicting the off-design arrodynamic performance of waveriders and waverider-based vehicles.

\subsubsection{Rocket-Based Combined-Cycle Engines}

A combined-cycle engine may be defined as any engire concept that incorporates two or more different engine cycles into one propulsion package. Hence, 
a multitude of different engine concepts may be developed by simple cornbination and permutation of existing engine cycles. The combined-cycle concept is largely used to develop an engine system that has the capability of producing efficient, positive thrust for a wide range of Mach numbers. As an example, a scranjet engine is expected to typically produce positive thrust for freestream Mach numbers greater than 6 to 8 . For Mach numbers lower than this, some other propulsion system may be incorporated into the engine design to produce positive, low-speed thrust, thus producing a combined-cycle concept.

A multitude of different combined-cycle concepts have been proposed over the ycars. Several examples are the air turbo ramjet engine, the licpuid air cycle engine (LACE), and the rocket-besed combined-cycle (RBCC) engine. The air turbo ramjet engine (an example schematic is shown in Fig. 1.10(a) from Heiser ct al. *8) consists of a fan-based compressor powered by a turbine which is spun by a gas generator system. Following the compressor, fuel is injected, mixed, and burned as is typical of a ranjet engine. The LACE engine (an oxample schematic is shown in Fig. 1.10(b) from Ref. 38) consists of an inlet which uses cryogenic fuel to liquify the incoming air. The liqueficd air is then pumped into a combustion chamber where it is combined with the prehcated fuel used to licuify the air. The mixture is then burned and the flow is expanded in a manner similar to a rocket engine.

An $\mathrm{RBCC}$ engine is the combination of a rocket-mode engine with any other type of ongine cycle. An example of an RBCC cngine is the supercharged ejector ramjet (SERJ) engine concept shown in Fig. 1.10(c) from Ref. 38. This particular SERJ concept consists of a fan-based compressor powered by a tip gas generator. Rocket flow adds to the fuel injected in the combustor section to entrain the incoming compressed air.

Early RBCC work was performed by the Marquardt Corporation. A rcview of 36 variations of the RBCC concept was analyzed in the Iate 1960's lyy Escher 


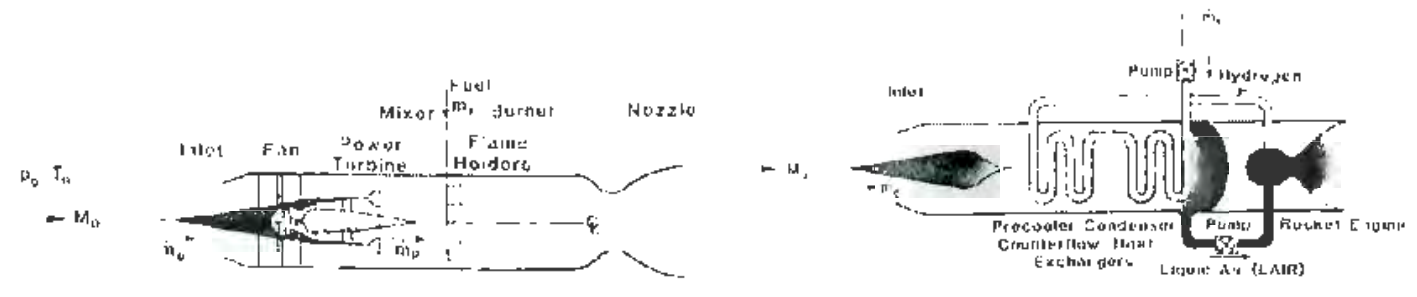

(a) Air turbo ramjet

(b) Liquid air cycle engine

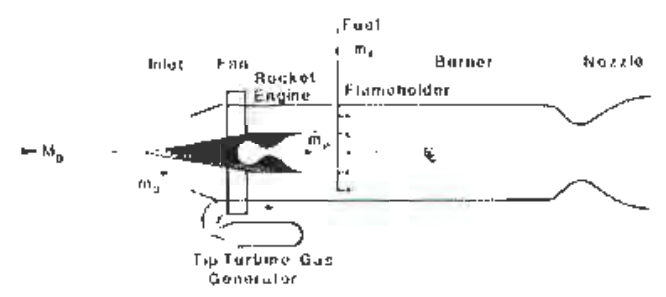

(c) Supercharged ejector ramjet

Figure 1.10: Examples of combined-cycle engine concepts [from Ref.38].

et al. In a latcr analysis, Foster ct al. ${ }^{40}$ refincs those 36 variations to 5 concepts and does an extensive analysis of cach concept. A history of the Marquardt Corporation's development of an RBCC engine concept is provided by Hyde et al. ${ }^{41}$

Curront interest in the RBCC engine concept revolves around its application to a single-stage-to-orbit (SSTO) vehicle system. Olds ${ }^{+2}$ has been studying the RBCC concept using a simplified systems level and weight analysis for multidisciplinary optimization studies. Aerojet has been developing the Strutjet cngine $^{13}$, a strnt-based RBCC engine designed for Mach 8 fighlit (see Fig. 1.11 from Ref. 43). The RBCC engine model used in the current work is loosely based on Acrojet's Strutjet engine. Finally, NASA Glonn Research Center has been developing the GTX SSTO vehicle concept ${ }^{2}$. The GTX volicle concept (shown in Fig. 1.12 from Rof. 2) is a vertical take-off, horizontal landing air-breathing 


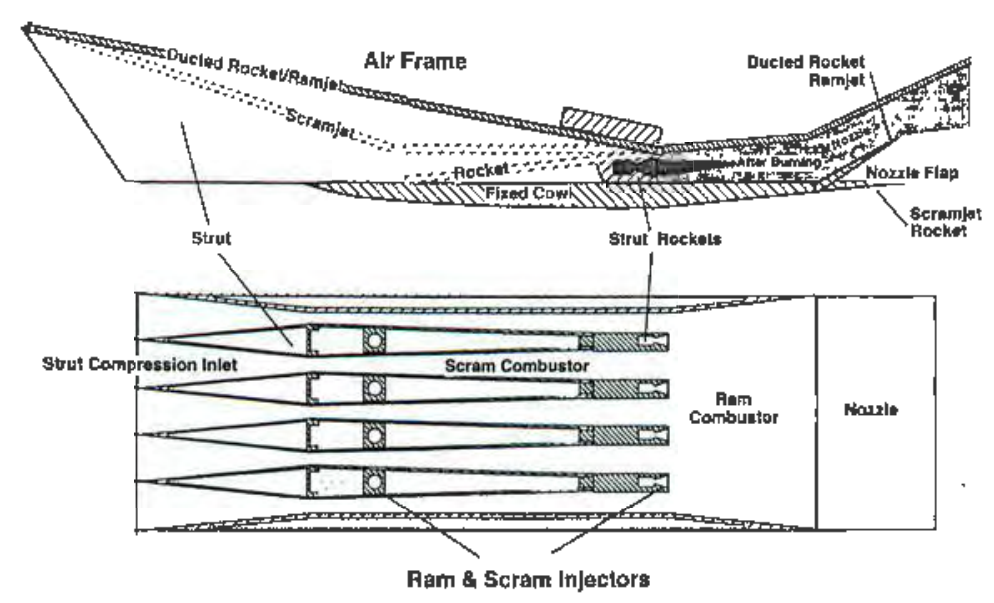

Figare 1.11: Acrojet's Strutjet engine [from Ref. 43].

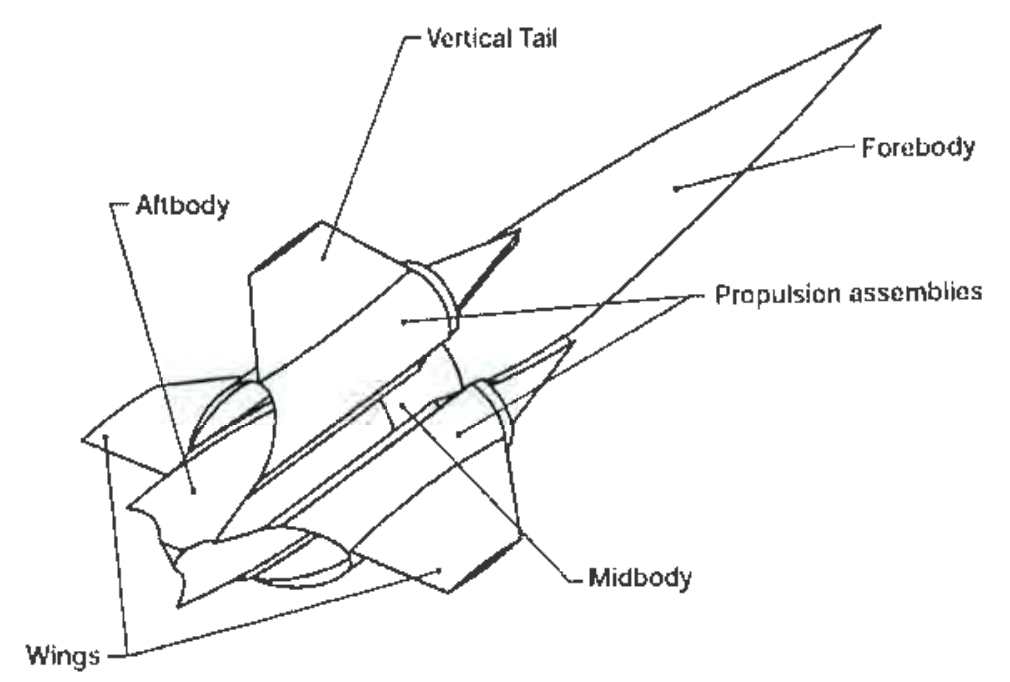

Figure 1.12: The GTX: an RBCC powered, SSTO vehicle concept currently under development at NASA Glenn Researcl Center [from Rof. 2]. 
vehicle concept with a payload capacity of 25,000 lbs.

\subsection{Present Work}

As stated in Sec. 1.1, the present work will incorporate an RBCC engine into an osculating cones waverider-based, engine-airframe integrated vehicle. The goal is to develop a rapid, analytical method for predicting the flowfield both on the vehicle fuselage and in the engine flow path. This goal involves developing analytical models for the off-design performance of waverider-based engine-airframe integrated vehicles over a Mach number range from low subsonic through high hypersonic. Also, to reach the stated goal, an analytical model of an RBCC engine in ramjet and scramjet modes will be produced both for the inlet compression system and the combustor sections. The models developed in this dissertation may prove useful for application into initial design study tools, trajectory performance algorithms, and/or optimization routines.

This work seeks to make the following contributions:

- Integration of an RBCC engine into an osculating cone waverider-based engine-airframe integrated vehicle.

- Development of rapid, analytical tools for predicting the aerodynamic distribution on the vehicle fuselage for both angles of attack and Mach numbers between subsonic and hypersonic.

- Rapid and detailed analytical modeling of an $\mathrm{RBCC}$ engine in ramjet and scramjet mode (including chemistry).

- Prediction and analysis of off-design aerodynamic performance of a waveriderbased vehicle. 
- Analysis of engine-airframe integration effects on off-design performance of a waverider-based vehicle. 


\section{Chapter 2}

\section{Airframe Geometry Definition}

The vehicle model to be presented is an extension of the hydrogen-fueled, scramjet-powered, osculating cones waverider vehicle class developed by Takashima $^{5}$. An example of this type of geometry is shown in Fig. 2.1 for a freestream design Mach number of 10. In this chapter, the methodology involved in creating the vehicle geometry and predicting it's on-design aerodynamic performance will be presented. Beginning at the leading edge of the vehicle, each major component of the vehicle geometry will now be discussed.

\subsection{Waverider Forebody}

The definition of the vehicle geometry begins with the forebody. The forebody used in this vehicle study was produced by the osculating cones waverider method, originally proposed by Sobieczky ${ }^{32}$. A waverider may be defined as any geometry that has inviscid shock wave attachment along its entire sharp leading edge.

As an example, a waverider geometry may be developed by using the inviscid, supersonic flow solution over a two-dimensional wedge. An example of this type of flowfield is shown in Fig. 2.2. To generate a waverider, trace a leading edge 


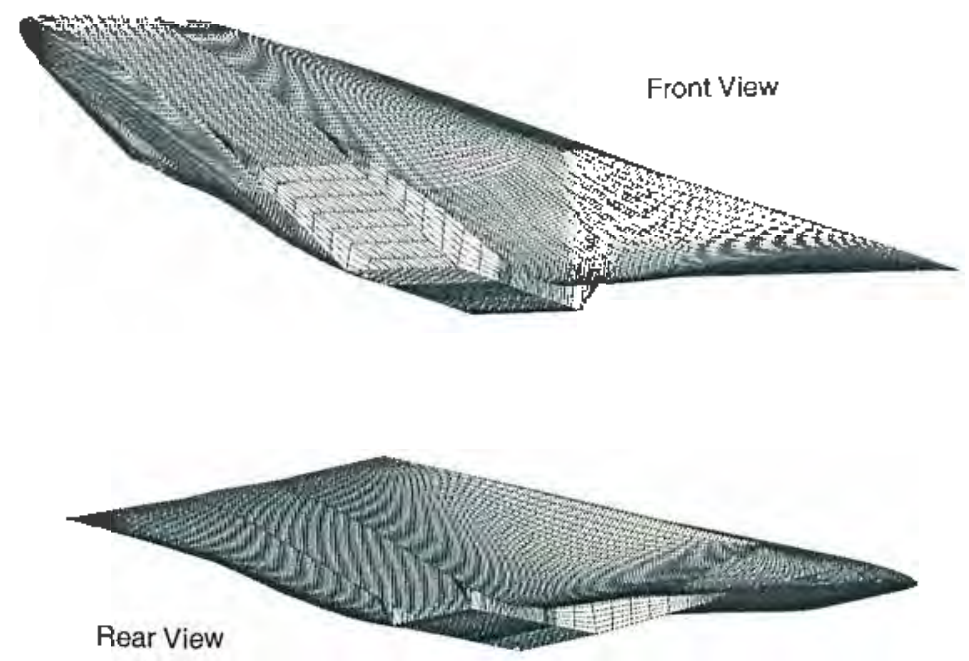

Figure 2.1: Two-view of exampie vehicle geometry $\left(M_{\infty}=10\right)$.

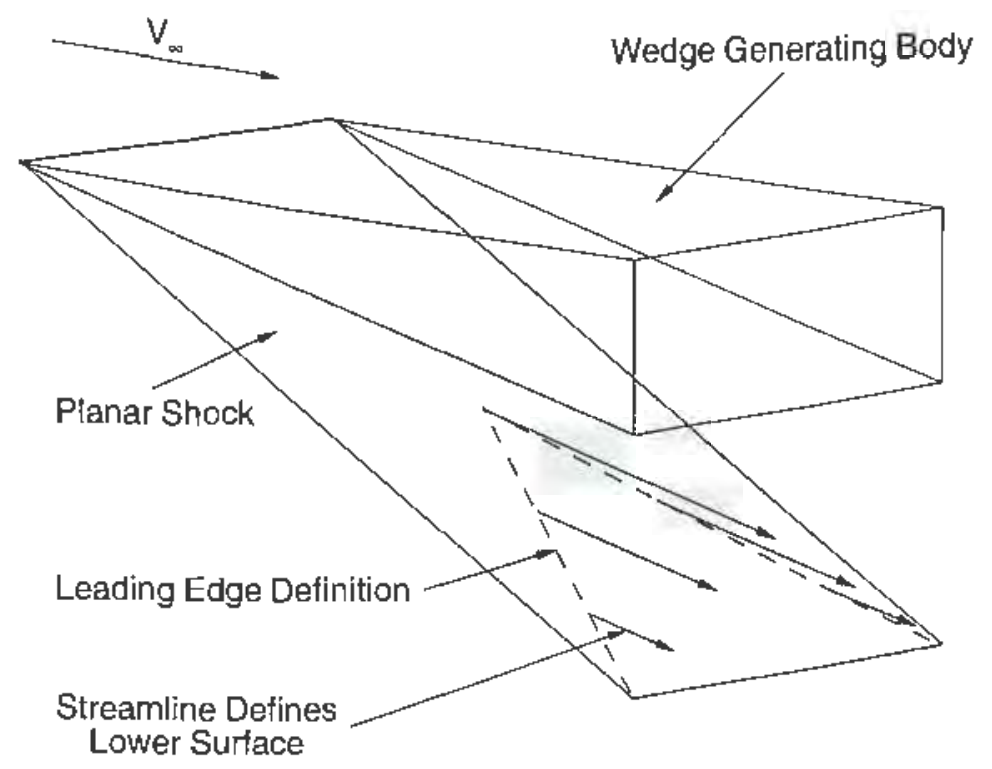

Figure 2.2: Example of waverider compression surface definition using the twodimensional, inviscid solution for supersonic flow over a wedge. 
shape along the shock wave generated by the wedge. At every point along the drawn leading edge, trace the streamline from the shock to the base plane of the waverider. Due to the hyperbolic nature of inviscid, supersonic flow, the set of streamlines drawn in the flow may also be seen as a solid surface. This solid surface becomes the lower compression surface of the waverider, and generates the entire shockwave located behind the leading edge. These geometries are seen to entirely "ride" on their own generated shockwave, hence the name "waverider". The upper surface of these geometries are typically taken as being parallel to the freestream velocity vector, thus having no effect on the lower surface of the waverider.

\subsubsection{Conical Flow Solution}

A waverider may be generated from a conical flow in the same manner as it was for a wedge flow as shown in Fig. 2.2. The solution to supersonic flow over a cone given the freestream Mach number and shock wave angle is expressed by the Taylor-Maccoll equation ${ }^{44}$

$$
\begin{aligned}
\frac{\gamma-1}{2}\left[V_{\max }^{2}-V_{r}^{2}-\left(\frac{d V_{r}}{d \theta}\right)^{2}\right] & {\left[2 V_{r}+\frac{d V_{r}}{d \theta} \cot \theta+\frac{d^{2} V_{r}}{d \theta^{2}}\right] } \\
& -\frac{d V_{r}}{d \theta}\left[V_{r} \frac{d V_{r}}{d \theta}+\frac{d V_{r}}{d \theta}\left(\frac{d^{2} V_{r}}{d \theta^{2}}\right)\right]=0
\end{aligned}
$$

where $V_{r}$ is the radial velocity component, $\theta$ is the angle from the axis of the cone to the ray of solution, and $V_{\max }$ is a theoretical velocity when the flow has been expanded to zero temperature found from

$$
V_{\max }=\sqrt{2 h_{o}}
$$

where $h_{o}$ is the freestream total enthalpy. The velocity component normal to the radial velocity may be found by

$$
V_{\theta}=V_{r}^{\prime}=\frac{d V_{r}}{d \theta}
$$


Defining a normalized velocity $\bar{V}$ as

$$
\bar{V}=\frac{V}{V_{\max }}
$$

the Taylor-Maccoll equation may be rewritten as

$$
\begin{gathered}
\chi\left[2 \bar{V}_{r}+\bar{V}_{\theta} \cot \theta+\frac{d^{2} \bar{V}_{r}}{d \theta^{2}}\right]-\bar{V}_{\theta}\left[\bar{V}_{r} \bar{V}_{\theta}+\bar{V}_{\theta} \frac{d^{2} \bar{V}_{r}}{d \theta^{2}}\right]=0 \\
\chi \equiv \frac{\gamma-1}{2}\left(1-\bar{V}_{r}{ }^{2}-\bar{V}_{\theta}^{2}\right)
\end{gathered}
$$

Rearranging Eq. 2.5 to solve for the second derivative of the radial velocity gives the following ordinary differential equation

$$
\frac{d^{2} \bar{V}_{r}}{d \theta^{2}}=\frac{\bar{V}_{r} \bar{V}_{\theta}^{2}-2 \chi \bar{V}_{r}-\chi \bar{V}_{\theta} \cot \theta}{\chi-\bar{V}_{\theta}^{2}}
$$

Recognizing that Eq. 2.7 may be written as

$$
\frac{d^{2} \bar{V}_{r}}{d \theta^{2}}=f\left(\theta, \bar{V}_{r}, \bar{V}_{\theta}\right)=f\left(\theta, \bar{V}_{r}, \bar{V}_{r}^{\prime}\right)
$$

the ordinary differential equation may be numerically integrated using the fourth order Runge-Kutta method ${ }^{45}$. Solution of the Taylor-Maccoll equation is calculated using an inverse process where the shock wave angle is given and the cone angle is then solved using the above technique. Details of the method for numerical solution of the Taylor-Maccoll equation may be found in Anderson ${ }^{46}$.

The solution for the flowfield over a cone is found as a function of $\theta$. The results are then placed into a cubic spline, so that any arbitrary value of $\theta$ may be given and the resultant flowfield property at the desired ray may be found. The splines of the cone data will be used when the streamlines of the flowfield (the lower surface of the waverider) are to be calculated. The data will also be used to find the pressure, temperature, velocity, and Mach number profiles on the forebody. 


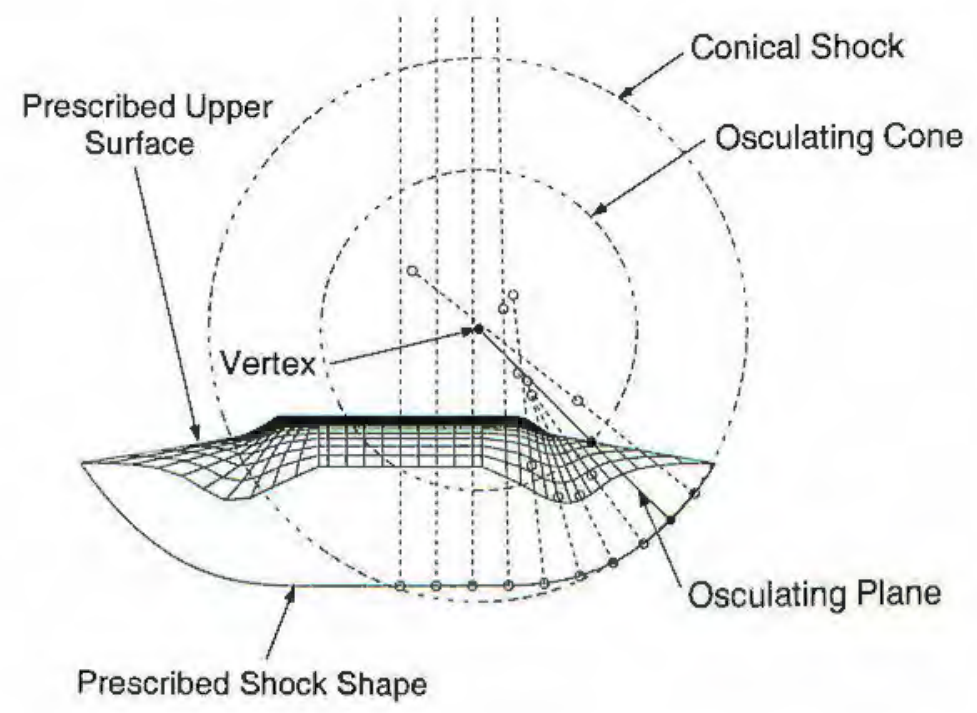

Figure 2.3: Osculating cone waverider development [from Ref. 5], cross-section of exit plane.

\subsubsection{Osculating Cones Waverider Method}

Any shock flowfield can be used to produce a waverider. For instance, a waverider based on conical flow may be developed from the supersonic solution for flow over a cone described above. As discussed previously, the leading edge of the waverider must be defined. From the leading edge, the lower surface is developed by tracing streamlines in the flowfield from the shock to the end of the waverider. The upper surface of the waverider is aligned parallel to the freestream.

For the osculating cones method described herein, two power-law curves $(y=$ $A x^{n}$ ) are prescribed: the top surface of the vehicle and the shape of the shock wave at the trailing edge of the waverider (see Fig. 2.3, from Takashima ${ }^{5}$ ). Also prescribed is the freestream Mach number and the shock wave angle on the cone. From this information, the conical flowfield may be solved as a function of ray angle from the methodology described in Section 2.1.1. 
The shock wave shape is used to determine at what location along the cone solution the streamline should be traced. At each location along the shock, the radius of curvature of the shock shape is found. The radius of curvature of the shock corresponds to the location in the cone solution where the shock has the same radius of curvature. The leading edge of the geometry may then be determined from tracing a straight line (parallel to the freestream flow) from the upper surface trailing edge to the shock intersection point. The intersection point is known from the prescribed shock angle. The streamline may then be traced from the leading edge to the trailing edge of the waverider (discussed in the next section). In this way, taking various points along the shock, the lower surface of the waverider may be developed. For more details on the methodology of developing osculating cones waveriders, see Takashimas.

An inherent assumption in the osculating cones method is that the azimuthal pressure gradients along the waverider geometry are negligible. While there are cases where this assumption is invalid, for the vast majority of waverider designs this assumption is valid due to their low surface curvature and high design Mach numbers. From the defined shape of the shockwave, the majority of the cone flow solutions are taken at locations on the cone which are quite distant from the apex. Thus, streamline curvature is small and pressure along the compression surface is fairly constant. In fact, Starkey ${ }^{47}$ determined that for a representative osculating cones waverider geometry, a wedge-derived waverider method gave excellent approximations (under $1 \%$ error) at on-design conditions.

\subsubsection{Streamline Tracing}

A surface streamline (for waverider compression surface generation) may be traced by combining the results of the cone solution cubic spline (described in Section 2.1.1) with the location of the leading edge with respect to the conical 
shock (described in Section 2.1.2). Assigning a length of the streamline in the freestream flow direction $l_{\text {stream }}$ and the number of points desired for describing the streamline $j_{\text {stream }}$, an incremental value of $\mathrm{x}$ may be found

$$
x_{\text {inc }}=\frac{l_{\text {stream }}}{j_{\text {stream }}-1}
$$

The streamline may then be traced from the starting point on the shockwave through the length of the surface streamline in the freestream flow direction. Starting with a point $a$ in the flowfield (see Fig. 2.4) located at an angle $\theta_{1}$ from the cone centerline, the velocity vector (found from the cubic spline) is followed to the point $b$ in the flowfield. The distance traveled between $a$ and $b$ is

$$
x_{b}-x_{a}=x_{\mathrm{inc}}
$$

The height of point $b$ is then found from the velocity vector information from the cubic spline of the cone solution

$$
y_{b}=y_{a}+x_{\text {inc }} \tan \left[\theta_{1}-\tan ^{-1}\left(\frac{V_{0}}{V_{r}}\right)\right]
$$

The angular location of point $b$ is then found from

$$
\theta_{2}=\tan ^{-1}\left(\frac{y_{b}}{x_{b}}\right)
$$

The flowfield properties at point $b$ are then found from the cubic spline of the cone solution for the value of $\theta_{2}$. In this manner, starting with the leading edge location on the shock as point $a$, the solution may then be traced through the cone flow solution until the end of the waverider is reached. Combining the solutions of all the leading edge points defined in Section 2.1.2, the waverider forebody compression surface is defined along with all pertinent aerodynamic data at on-design conditions.

The compression surface streamline of the waverider will most likely need to be oriented at a dihedral angle due to the shock curvature (see the streamline 


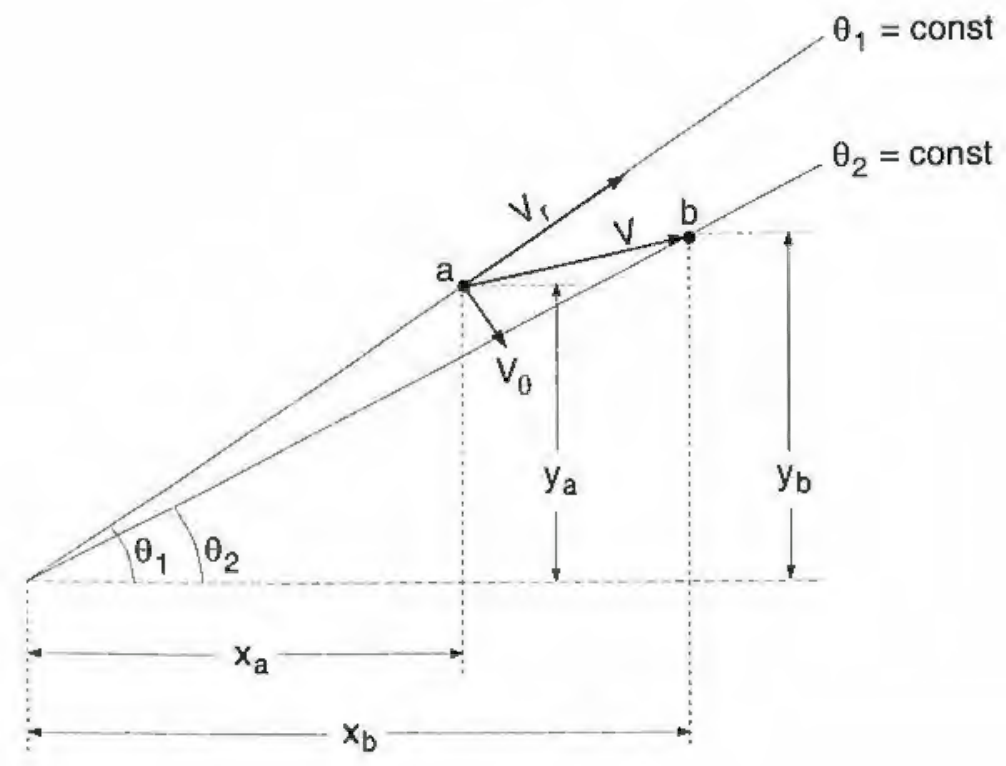

Figure 2.4: Generation of body contour from streamline tracing.

labeled "osculating plane" in Fig. 2.3 for an example of a streamline with dihedral). Thus, the coordinates $x$ and $y$ solved above will need to be transformed from streamline orientation to the Cartesian coordinate system of the vehicle.

\subsubsection{Examples Using the Osculating Cones Waverider Method}

The flexibility of the osculating cones method may now be demonstrated by showing several solutions for waverider shapes. The first solution using the osculating cones method is the "caret"-wedge style waverider shown in Fig. 2.5 along with the generating shock wave shape. This style of waverider geometry is generated using a wedge flowfield. The osculating cones method creates these geometries by assigning an infinite radius of curvature to the shockwave, thus creating a planar or wedge-derived shock. An inherent disadvantage to these shapes is their lack of usable volume, especially near the leading edges. While these shapes have high inviscid $L / D$, they suffer from high viscous drag with respect to their volume because of their large surface area. The volume that 


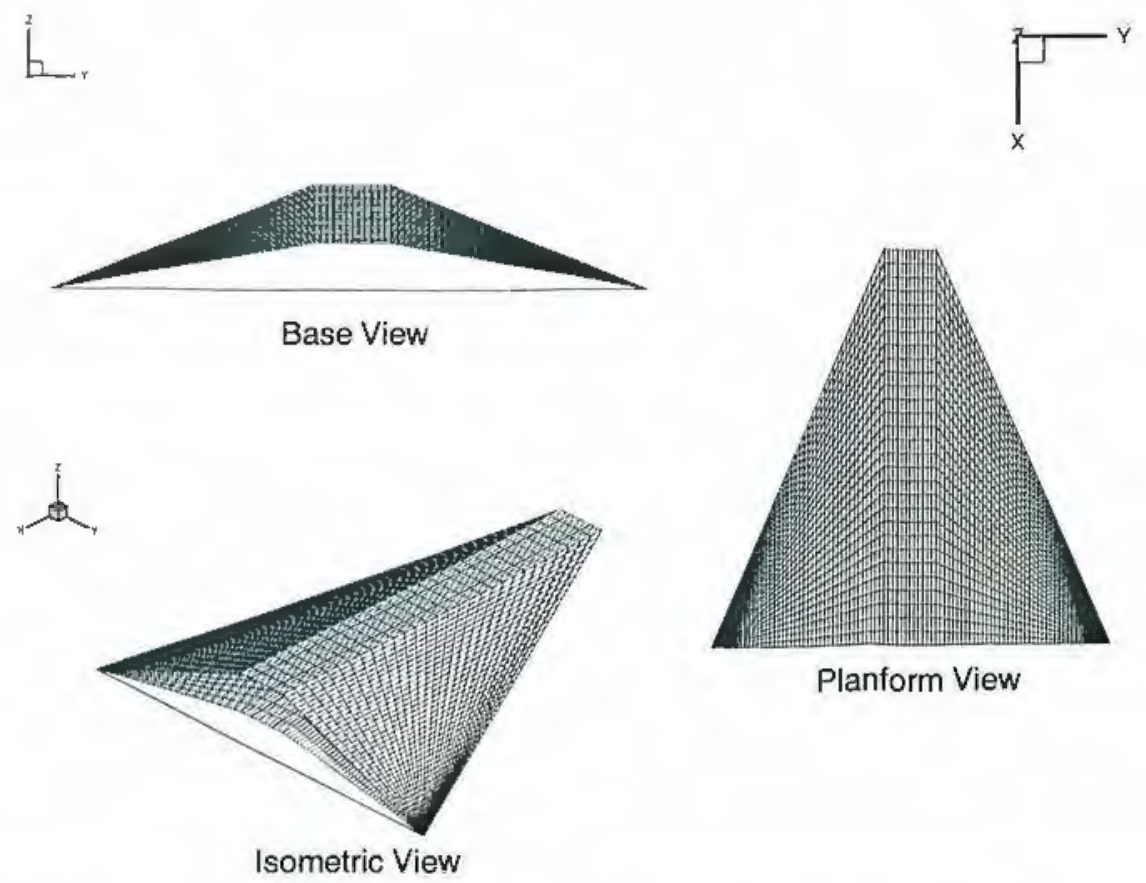

Figure 2.5: Caret waverider generated using osculating cones method.

these shapes have does not easily lend itself for useful storage space for fuel tanks or other payloads. However, an advantage to these geometries is the uniform, two-dimensional flowfield between the waverider and the shock wave. This feature may be advantageous for flow entering into a scramjet combustor as well as minimizing detrimental three-dimensional viscous effects (such as early boundary layer separation).

Better volume packaging may be achieved by using a fully conical flowfield. A conically derived waverider shape calculated using the osculating cones method is shown in Fig. 2.6 along with its generating shock wave shape. These shapes are created by assigning a constant, finite, radius of curvature to the defined shock wave. An immediate advantage seen in these shapes is a greater concentration of volume near the center of the body, with less volume in the leading edge area. Thus, more accessible volume is available for storage purposes. However, the flowfield on these shapes is conical, hence the flow between the waverider and 
bـ
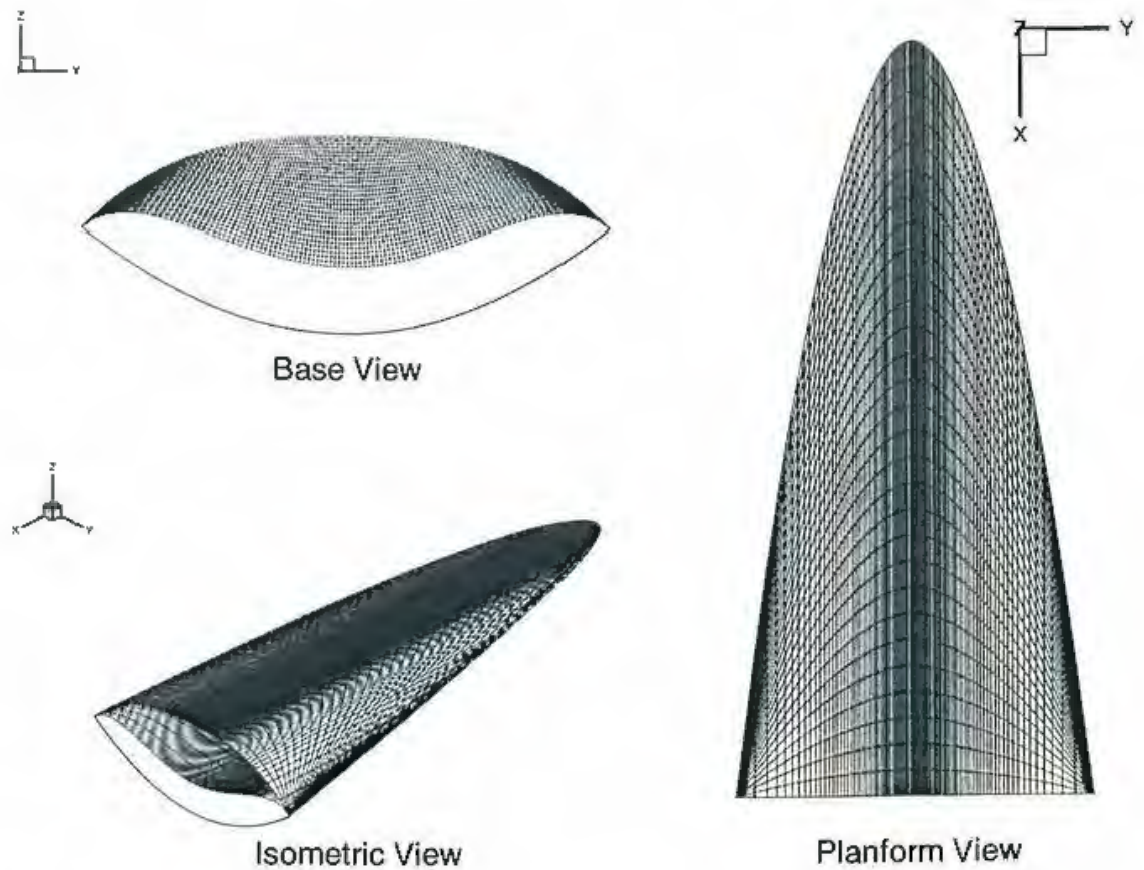

Figure 2.6: Conical waverider generated using osculating cones method.

the shock varies in the radial direction. Thus, nonuniform flow will enter the engine, which may cause problems with the engine design.

The osculating cones method is used to capture the conical properties of highly accessible volume and the wedge properties of flow uniformity (especially in the region near the engine inlet). This method allows the user to define the shock wave shape, thereby selecting the flowfield generated by the waverider geometry. Thus, a section near the centerline of the vehicle with flow uniformity may be generated by selecting a shock wave shape with infinite radius of curvature in this region. Further away from the centerline, the shock wave may be curved, thus allowing for more accessible volume regions. An example of a vehicle calculated with the osculating cones technique is demonstrated in Fig. 2.7, along with its generating shock wave shape. Flow uniformity exists in the centerline region due to the straight portion of the shockwave. Away from the centerline, the forebody has a large region of accessible volume concentrated away from the 


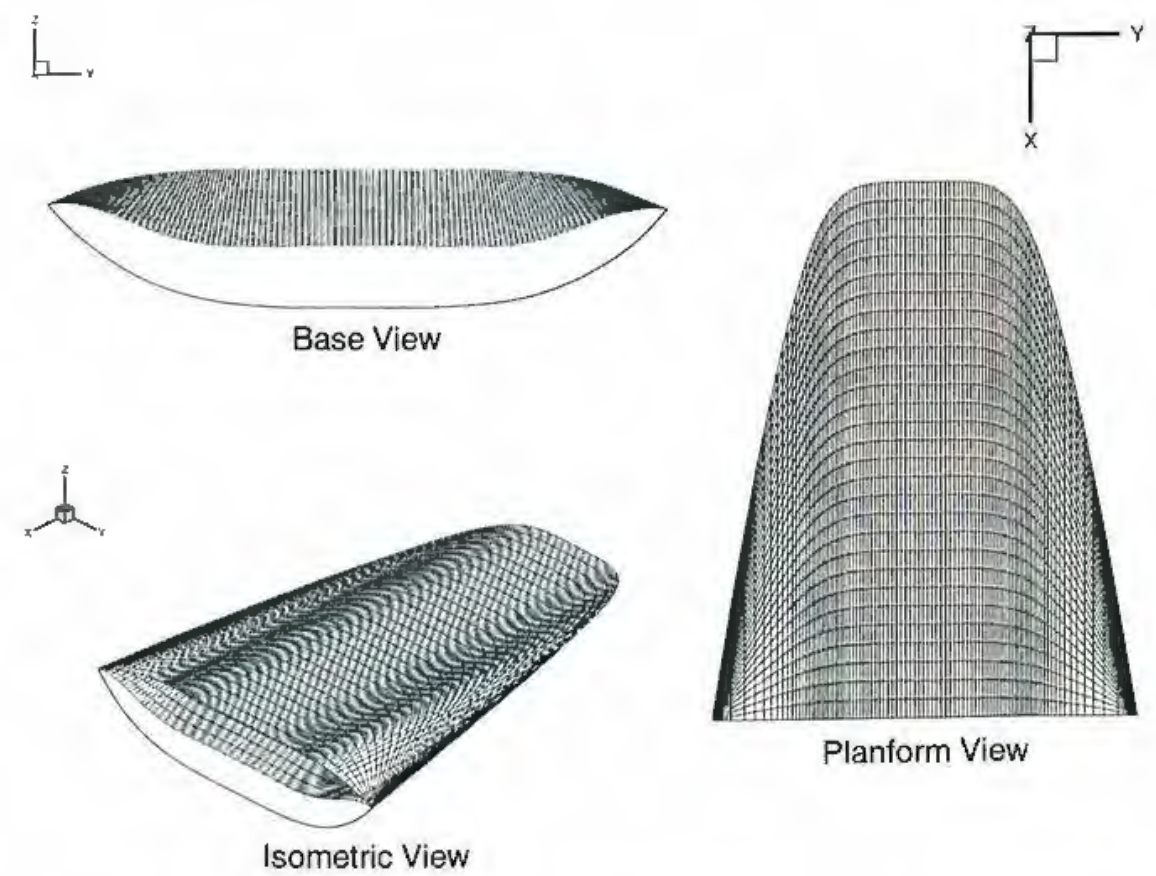

Figure 2.7: An example of an osculating cones waverider.

leading edges. Thus, the osculating cones method has the advantages of both wedge and conical flow.

\subsection{Engine Inlet}

The engine inlet geometry for the vehicle is shown in Fig. 2.8. The engine inlet consists of four wedge compression flowfields. The first wedge compression surface is the centerline flowfield from the osculating cones forebody. The other three wedges all have the same compression angle $\theta_{w}$. The engine inlet height $H_{\text {eng }}$ is assumed to be a constant value for manufacturing concerns. The lengths of each of the inlet compression ramps $\left(L_{2}, L_{3}, L_{4}\right)$ are selected so that the inlet shock waves all intersect with the bow shock wave at a single point for the on-design condition. The leading edge of the engine cowl is then placed at this intersection point so that the resulting engine cowl shock is cancelled at the 

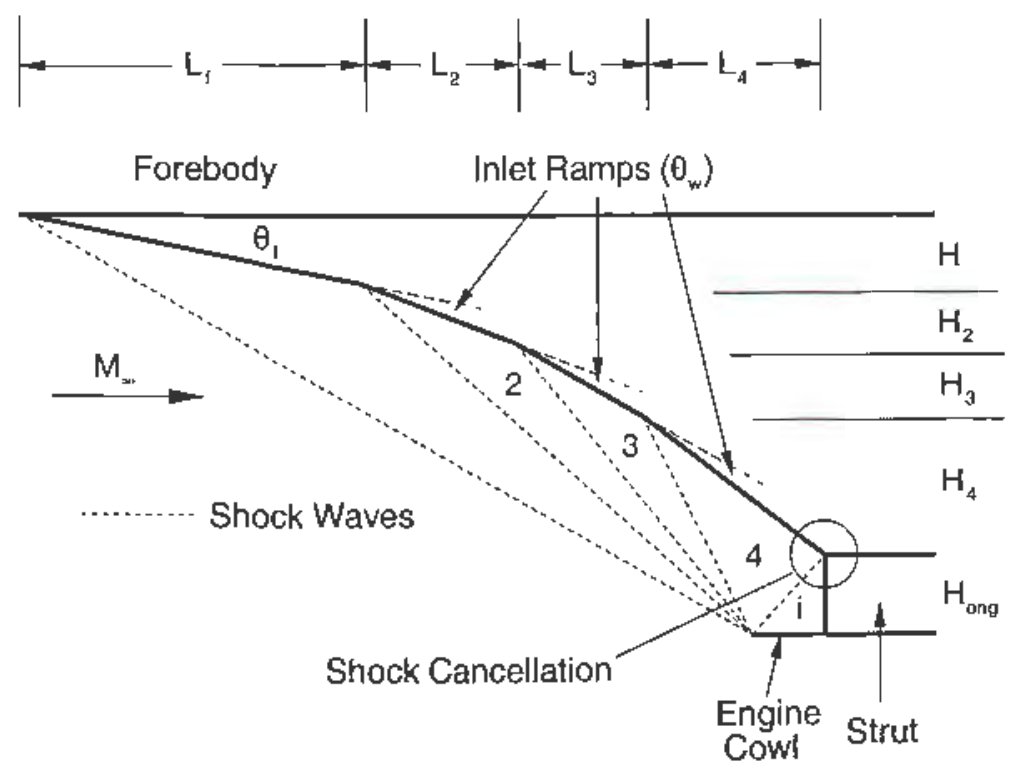

Figure 2.8: Vehicle inlet geometry.

top of the engine (sce Fig. 2.8). Any shock/shock interactions that may occur due to leading edge blunting on the cowl are ignored for this study. Also, any slock wave/boundary layer interactions, which could result in boundary layer separation as woll as flow unsteadiness, are also neglected. The RBCC engine struts are located inmodiately following the shock cancellation point.

The engine cowl leading edge is located in the $\mathrm{x}$ and $\mathrm{y}$ directions by:

$$
\begin{gathered}
x_{\text {cow } 1}=L_{f} \frac{\tan \theta_{f}-\tan \left(\beta_{2}+\theta_{f}\right)}{\tan \beta-\tan \left(\beta_{2}+\theta_{f}\right)} \\
y_{\text {cowl }}=x_{\text {cow }} \tan \beta
\end{gathered}
$$

where $x_{\text {cowl }}$ and $y_{\text {cowl }}$ are the $x$ and $y$ locations of the cowl leading edge at ondesign conditions, $L_{f}$ is the length of the waverider forcbody, $\theta_{f}$ is the wedge angle of the waverider forebody, $\beta$ is the bow shock wave angle, and $\beta_{2}$ is the shock wave angle off of the first wedge ramp inlet with respect to the inlet. The solution for the inlet iterates on ramp angle $\theta_{w}$ to hind the ramp lengths $L_{2}, L_{c_{3}}$, and $L_{4}$ necessary to meet the desired on-design engine conditions given 
previously. For a given value of $\theta_{w}$, the ramp height $H_{2}$ and length $L_{2}$ for the first ramp may be calculated

$$
\begin{gathered}
H_{2}=\frac{\left(x_{\text {cowl }}-L_{f}\right)}{\frac{\tan \left(\theta_{1}+\beta_{3}+\theta_{w}\right)}{\frac{\tan \left(\theta_{1}+\beta_{3}+\theta_{w}\right)}{\tan \left(\theta_{1}+\theta_{w}\right)}-1}-y_{\text {cow } 1}+H} \\
L_{2}=\frac{H_{2}}{\tan \left(\theta_{1}+\theta_{w}\right)}
\end{gathered}
$$

whore $H$ is the height of the waverider forcbody. The ramp height $H_{3}$ and length $L_{3}$ for the second ramp may now be solved

$$
\begin{gathered}
H_{3}=\frac{\left(x_{\operatorname{cow}}-L_{f}-L_{2}\right) \tan \left(\theta_{1}+\beta_{4}+2 \theta_{w}\right)-y}{\frac{\tan \left(\theta_{1}+\beta_{4}+2 \theta_{w}\right)}{\tan \left(\theta_{1}+2 \theta_{w}\right)}-1}-H+H_{2} \\
L_{3}=\frac{H_{3}}{\tan \left(\theta_{1}+2 \theta_{w}\right)}
\end{gathered}
$$

The distance between tho leading edge of the engine cowl and the shock cancellation point on the inlet is

$$
x_{\text {cancal }}=\frac{H_{\text {ong }}}{\tan \left(\beta_{i}-\theta_{1}-30_{w}\right)}
$$

The engine height $H_{\text {eng }}$ remains constant to match the engine specifications of the Aerojet Strutjet engine. The leng'th $L_{4}$ and height $H_{4}$ of the final inlet ramp is

$$
\begin{gathered}
L_{4}=x_{\text {cowl }}+x_{\text {calcal }}-L_{J}-L_{2}-L_{3} \\
H_{4}=L_{4} \tan \left(\theta_{1}+3 \theta_{w}\right)
\end{gathered}
$$

The solution is then iterated using the secant method (Appendix A) to find

$$
y_{\text {cowl }}-H-H_{2}-H_{3}-H_{4}-H_{\text {cing }}=0
$$

Whon Eq. 2.22 is satisfied, the conditions behind the final shock wave are taken as the incoming conditions into the engine. It is notod that the change in the ratio of specific heats $\gamma$ due to temperature rise is accounted for in the inlet 


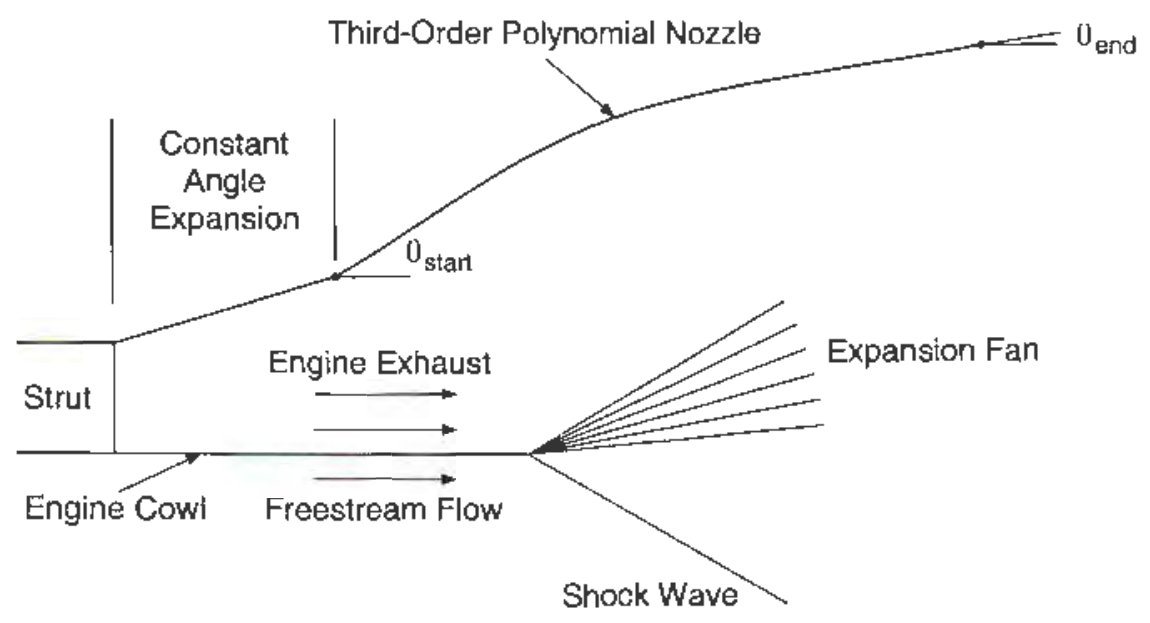

Figure 2.9: Vehicle nozzle geometry.

calculations (from CHEMKIN II ${ }^{49}$ ). The value of $\gamma$ behind each shock wave is taken as the incoming value of $\gamma$ for the next shock wave. Due to modeling of the change in $\gamma$ through the inlet, there is a small but negligible error in mass How on the order of $1-2 \%$.

\subsection{Nozzle}

The nozzle of the vehicle (see Fig. 2.9) consists of three sections: an interior constant angle expansion, an interioz nozzle, and an exterior (plug) nozzle. The interior constant angle expansion is assumed to still lave chemical renctions occurring from the exhanst of the constant area combustor section, located between each of the struts. The flowfield in this region is calculated using the engine combustor code discussed in Section 3.2. The angle of the cxpansion region is held at a constant, arbitrary value of 8.9 degrces. This angle was selected to loosely model Aerojet's Strutjet cngine ${ }^{13}$ and implics no optimality.

Following the constant angle expansion region, a third-order polynomial is used to model the nozzle geometry (which also acts as the lower surface centerline 


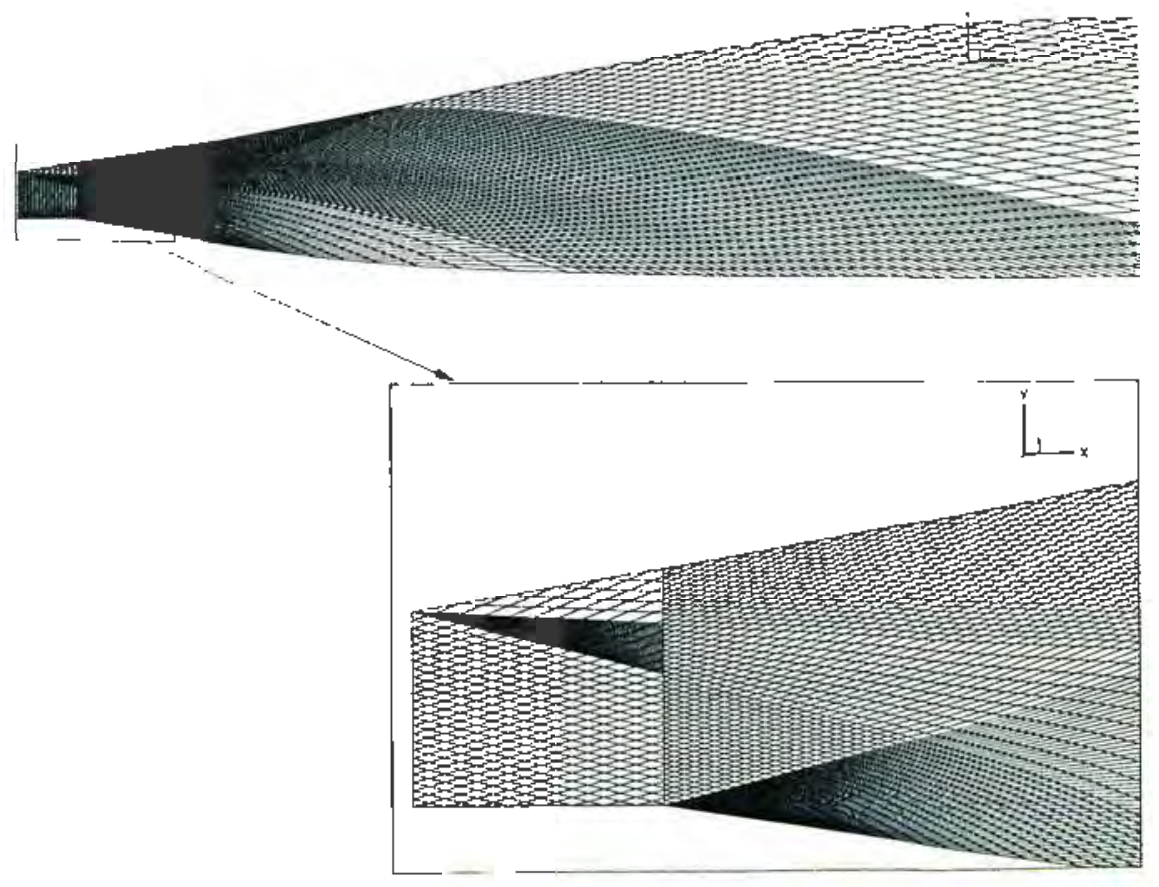

Figure 2.10: Characteristic mesh of volicle nozzle.

of the vehicle). The coefficients of the polynomial are determined from the location of the beginning of the nozzle, the assigned slope at the beginning of the nomzle, the assigned location of the end of the velicle, and the assigned slope of the nozzle at the end of the vehicle (see Fig. 2.9 for details). The cubic nozzle geometry is split into two regions: an interior nozzle and an exterior nozzle. The separation of these two regions occurs at the end of the engine cowl. Selecting a large engine cowl length provides a large internal nozzle which will maintain higher pressure, resulting in higher lift and thrust. However, this increase in lift and thrust comes at the cost of high viscous drag due to the high temperature gas on the engine cowl.

Both the internal and oxternal nozzle flows are calculated using the twodimensional method of characteristics ${ }^{*}$, assuning no chemical rcactions (i.e., the flowfield is considered to be frozen at the ond of the constant angle expansion). A representativo characteristic mesh developed using this method is shown 


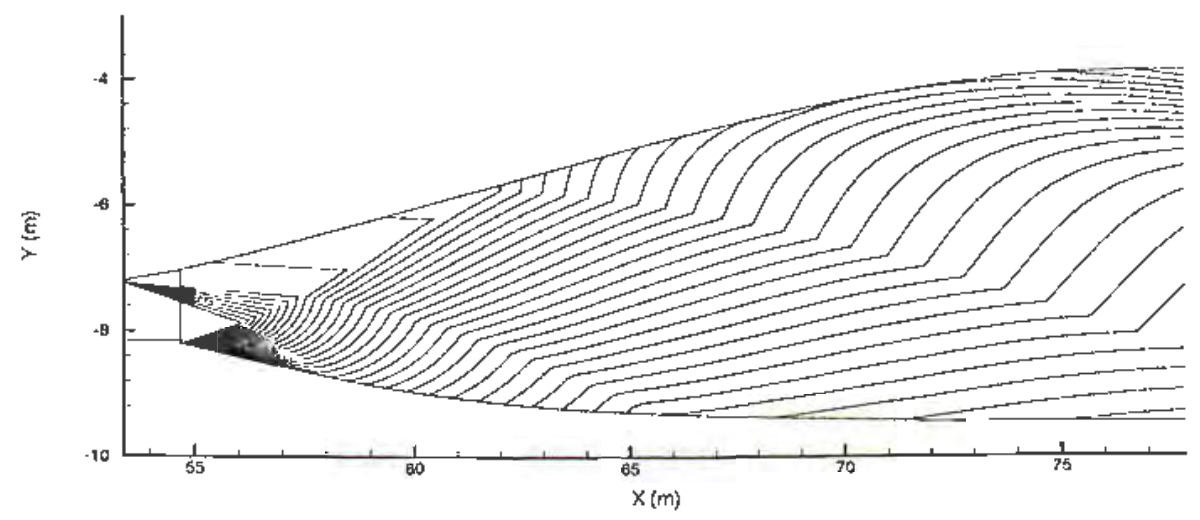

Figure 2.11: Contours of Mach number on a vehicle nozkle $\left(M_{\text {comb }}=4.13\right)$.

in Fig. 2.10 for an cxample nozzle geometry. The internal nozzle scction is calculated first, using the exit conditions from the constant angle internal cxpansion. 'This solution is calculated to the end of the engine cowl. The incoming flow for the external nowzle is found from an interpolation of the flow loaving the internal nozzle section. To account for the mismatch in pressure between the flow exiting the engine and the freestream conditions, a shock is added on the lower stirface of the engine cowl, and an expansion (represented as the characteristic mesh emanating from the edge of the engine cowl) is added to the upper surface as shown in Fig. 2.9. The conditions resulting from the shock and expansion are iterated to match pressure and flow direction. From this, the final (or terminating) characteristic from the engine cowl may be developed. The exterior nozzle flowfield is then solved using the nethod of characteristics as long as the teminating characteristic does not intersect with the surface of the vehicle. In this way, the pressure distribution along the centerline of the lower surface of the vohicle is solved. An example solution for Mach number distribution in a representative nozzle is shown in Fig. 2.11 for an enginc cxit Maxh number of 4.13. 


\subsection{Upper and Lower Aft Body Sections}

Aft of the forebody, the fusclage shape is developed from a serics of thirdorder polynomials in the streanwise direction to maintain continuous slope along the length of the vehicle. The upper aft section third-order polynomial coefficients are cletemined from the location and slope at the ond of the upper surface of the Forebody and from the location and slope at the end of the velicte. The trailing cdgo is assumed to be closed, with no curvature in the direction normal to the frostrean at zero roll angle. Trailing edge closure is enforced to minimize the effects of base area on transonic performanec. The lower aft section is composed of two third-order polynomial sections. The first polynomial goes from the end of the Iower surface of the waverider forebody to the end of the constant angle expansion in the engine. The sccond third-order polynomial then strotches from the end of the constant angle expansion section to the end of the veinicle. The flowficld on the fuselage is then solved using shock-expansion thoory ${ }^{19}$. Detalis of the methodology in determining the fusclage shape naay be found in Takashima ${ }^{5}$.

\subsection{Side Walls and Engine Cowl}

The side walls of the engine are extended both fore and aft of the combustor section to maintain pressure containmont in the compression and expansion regions. The engine side wall begins at the beginning of the first inlet compression ramp. The angle of the sidewall fron its starting location to the engine inlet cowl is based on the on-design shock angle coming off of the hrst inlet compression ramp. At the end of the engine, the side wall is extended a short distance to partially maintain the pressure in the expansion region, thereby incroasing lift and thrust. The angle of the side wall aft of the engine oxit is kept at an 
arbitrary value of 45 degrees.

Flow properties on the exterior side of the side walls are assumed to be a linear average between the properties on the fuselage at the top of the side walls, and the freestream properties based on the flow exiting the waverider forebody on the bottom of the side walls. Flow properties on the interior of the side walls are based on the compression flowheld from the inlet ramps, the compression flowfield from the RBCC struts, the flowfield in the combustor, and the expansion flowfield from the nozzle. Flow streamlines on both the exterior and interior of the side walls are assumed to run parallel with the side walls to aid in prediction of viscous drag.

The exterior surface of the engine cowl is assumed to have purely twodimensional flow. The flow properties on the exterior of the engine cowl are calculated using oblique shock theory or Prandtl-Meyer flow, depending upon the Mach number and the angle of attack of the vehicle. At on-design Mach number and zero angle of attack, the exterior of the engine is assumed to be at freestream conditions. The interior surface of the engine cowl is calculated based on the compression flowfield from the RBCC struts, the combustor flowfield, and the expansion flowfield from the nozzle. Streamlines are assumed to run parallel to the engine cowl for estimation of viscous drag.

Both the engine side walls and the engine cowl are assumed to be infinitely thin. This assumption neglects the structural integrity of the side walls from the pressure forces exerted from the compression and combustion regions. However, giving these walls finite thickness means that shocks and expansions from these walls will affect the flowfield on the vehicle, thus making analysis more difficult. Also, the model accounts for viscous drag, but viscous effects such as shock wave/ boundary layer interactions and corner flow are not considered. These effects may be important in the detailed design of the combustor, but are neglected for this order of analysis. 


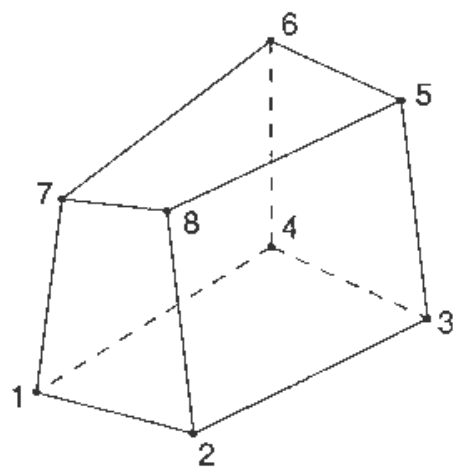

Figure 2.12: Example volume eloment.

\subsection{Airframe Properties}

\subsubsection{Volume Calculation}

The volume of the vehicle is determined numerically by splitting the vehiclo grid into a series of tetrahedron shapes. Figure 2.12 shows how four adjacent points on the freestream surface of the waverider forebody may be connected to four corresponding points on the compression surface. The six sided shape in Fig. 2.12 may then bo split into six tetrahedron shapes, as shown in Fig. 2.13. The volume of a tetrahedron with vertices $\left(x_{1}, y_{1}, z_{1}\right),\left(x_{2}, y_{2}, z_{2}\right),\left(x_{3}, y_{3}, z_{3}\right)$, and $\left(x_{1}, y_{1}, z_{4}\right)$ may be calculated exactly by ${ }^{50}$

$$
\mathrm{Vol}= \pm \frac{1}{6}\left|\begin{array}{llll}
x_{1} & y_{1} & z_{1} & 1 \\
x_{2} & y_{2} & z_{2} & 1 \\
x_{3} & y_{3} & z_{3} & 1 \\
x_{4} & y_{4} & z_{4} & 1
\end{array}\right|
$$



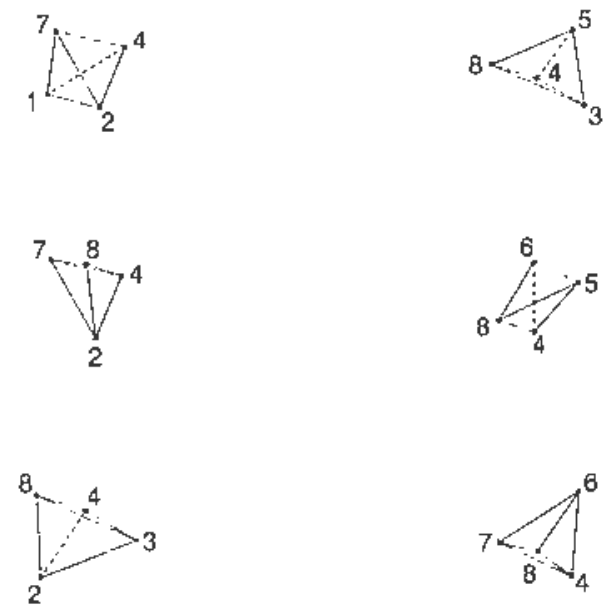

Figure 2.13: Splitting of volume element in Fig. 2.12 into six tetrahedrons.

where \pm is used to insure positive volune values. Summing the volume of all tetrahedrons then gives the volutne of the entire vehicle.

\subsubsection{Friction Coefficient and Shear Stress}

Due to the large range of Mach numbers and angles of attack to be solved, the characteristics of flow transition from laminar to turbulent, are unknown on the volicle. Hence, as a conservative estimate, all viscous calculations assume the flow on the entire vehicle is fully turbulent. The streamlines of the flowfield are assumed to run along the gridlines of the veliche model in order to calculate the distance the flow has traveled. The friction coefficient is calculated using Eckert's reference temperature method ${ }^{51}$

$$
C_{f}=\frac{0.0592}{\left.(\operatorname{Re})_{x}^{*}\right)^{0.2}}
$$

where $R e_{x}^{*}$ is the Reynold's number evaluated at the reference temperahure $T^{*}$

$$
T^{*}=T_{e}\left(0.5+0.039 M_{e}^{2}+0.5 \frac{T_{w}}{T_{e}}\right)
$$


and $T_{e}$ is the temperature at the edge of the boundary layer (the inviscid temperature), $M_{e}$ is the Mach number at the enge of the boundary layer, and $T_{w}$ is the wall tomperature. The wall temperature is assumed to be a constant value of $300 \mathrm{~K}$ below Macis 2, a lincar function between Mach 2 and Mach 12, and a constant value of $1200 \mathrm{~K}$ above Mach 12 . The Reynold's number evaluated at the reforence temperature is

$$
R e_{x}^{*}=\frac{\rho^{*} V_{e} x}{\mu^{*}}
$$

where $p^{*}$ is the density evaluated at the reference temperature (from the perfect gas law), $V_{z}$ is the velocity at the edge of the boundary layer, $x$ is the distance traveled and $\mu^{*}$ is the viscosity coefficient evaluated at the reference temperature. The viscosity coefficiont is calculated using Sutherland's law for air ${ }^{\text {ts }}$

$$
\mu^{*}=1.789 \times 10^{-5}\left(\frac{T^{*}}{288}\right)^{\frac{3}{2}} \frac{398}{T^{*}+110}
$$

where $\mu$ and $T$ are in units of $\frac{k g}{m \cdot s}$ and Kelvin, respectively. The wall shear stress $\tau_{u}$ may thon be calculated by

$$
\tau_{w}=\frac{1}{2} \rho_{e} V_{e}^{2} C_{f}
$$

where $p_{e}$ is the density at the edge of the boundary layer.

\subsubsection{Heat Transfer}

Heat transfer to the walls is accounted for in the combustor model presented in Chapter 3 . The Stanton number $C_{H}$ may be written as

$$
C_{I I}=\frac{\dot{Q}^{\prime \prime}}{\rho U\left(h_{\mathrm{uw}}-h_{i v}\right)}
$$

Knowledge of the friction coefhicient allows for calculation of the combustor wall heat transfer using the turhulent form of Reynold's analogy

$$
C_{H}=\frac{C_{f}}{2 P r^{2 / 3}}
$$


where $P r$ is the Prandtl number (assumed to be 0.71 in air) and $T_{a w}$ is the adiabatic wall temperature calculated by

$$
T_{a w}=T\left(1+\frac{\gamma-1}{2} \operatorname{Pr}^{1 / 3} M^{2}\right)
$$

The heat transfer rate per unit area is then found from combining Eqs, 2.29 and 2.30

$$
\dot{Q}^{\prime \prime}=\frac{C_{f} \rho U c_{p}\left(T_{a w}-T_{w}\right)}{2 P r^{2 / 3}}
$$

where the wall enthalpies are assumed to be a function of the frestream specific heat at constant pressure and the wall and adiabatic wall temperatures. Integrating Eq. 2.32 yields

$$
\dot{Q}=\int_{0}^{l} \frac{2 C_{f} \rho U c_{p}\left(T_{a w}-T_{w}\right) A}{\mathcal{D} P r^{2 / 3}} d x
$$

where $\mathcal{D}$ is the hydraulie diameter defined as

$$
\mathcal{D} \equiv \frac{4 A}{P_{w}}
$$

where $P_{w i}$ is the wetted perimeter of the engine section. Equation 2.33 may be used in conjunction with the combustor model to estimate the heat transferred from the combustor flowfeld to the engine walls.

\subsubsection{Vehicle Mass}

The mass of the vehicle is broken into two parts: mass of the propellant and mass of the vehicle structure. The mass of the propellant may also be broken into two parts: mass of liquid hydrogen and mass of liquid axygen (small compared to mass of liquicl hydrogen). By assigning a propellent volume fraction $\pi_{p}$, the mass of the vehicle is calculated by

$$
m_{\mathrm{vel} 1}=\rho_{p} \pi_{p} \mathrm{Vol}+\rho_{\mathrm{veh}}\left(1-\pi_{\mathrm{p}}\right) \mathrm{Vol}
$$


where $m_{\text {veh }}$ is the mass of the vehicle, $\rho_{p}$ is the density of the propellant and $\rho_{\text {vel }}$ is the average density of the vehicle structure. Breaking apart the propellant mass, the mass of the vehicle may be stated as

$$
m_{\mathrm{veh}}=\rho_{\mathrm{O}_{2}} \pi_{\mathrm{O}_{2}} \pi_{p} \mathrm{Vol}+\rho_{\mathrm{H}_{2}}\left(1-\pi_{\mathrm{O}_{2}}\right) \pi_{\mathrm{p}} \mathrm{Vol}+\rho_{\mathrm{veh}}\left(1-\pi_{\mathrm{p}}\right) \mathrm{Vol}
$$

where $\rho_{\mathrm{O}_{2}}$ and $\rho_{\mathrm{H}_{2}}$ are the liquid densities of oxygen and hydrogen and $\pi_{\mathrm{O}_{2}}$ is the fraction of $\pi_{p}$ that is composed of oxygen. The propellant volume fraction is assumed to be $55 \%$. The liquid density of oxygen at $77.6 \mathrm{Kelvin}$ is $1230 \mathrm{~kg} / \mathrm{m}^{3}$ and the liquid density of hydrogen at 14 Kelvin is $75.9271 \mathrm{~kg} / \mathrm{m}^{3}$.

The average vehicle density is assumed to be $142.83 \mathrm{~kg} / \mathrm{m}^{3}$ for a Mach 12 hydrogen fueled vehicle ${ }^{11}$. This number is an estimate from a vehicle mass calculation performed by Ardema ${ }^{52}$ on a Mach 6 waverider vehicle with hydrogen fuel. An additional $15 \%$ was added to the original density calculation to account for additional mass from thermal protection systems for the current Mach number.

\subsubsection{Area and Force Calculation}

A surface element for the vehicle geometry is shown in Fig. 2.14. The surface element is split into two triangles (one of which is shaded in Fig. 2.14). The wetted surface area $A_{w}$ of the shaded triangle is calculated by

$$
A_{w}=0.5|\vec{C}|=0.5|\vec{A} \times \vec{B}|
$$

where vectors $\vec{A}, \vec{B}$ and $\vec{C}$ are defined as shown in Fig. 2.14. The planform area $A_{\text {plan }}$ and cross-sectional area $A_{\mathrm{cs}}$ may be calculated by

$$
\begin{gathered}
A_{\text {plan }}=A_{w} \frac{C_{y}}{|\vec{C}|} \\
A_{\mathrm{cs}}=A_{w} \frac{C_{x}}{|\vec{C}|}
\end{gathered}
$$




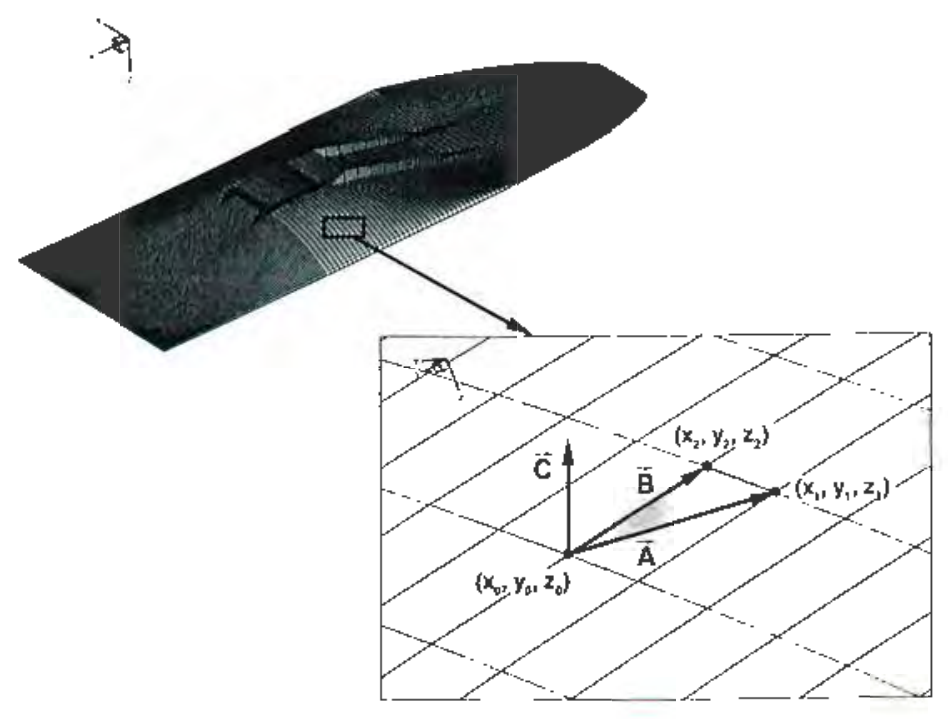

Figure 2.14: Surface eloments for area and force calculations.

where $C_{x}$ and $C_{y}$ are the $x$ and $y$ components of $\vec{C}$.

The average prossure $p_{\text {avg }}$ and average shear stress $\tau_{\text {avg }}$ on the triangle element are calculated by

$$
\begin{aligned}
& p_{\mathrm{avg}}=\frac{p_{0}+p_{1}+p_{2}}{3} \\
& \tau_{\mathrm{avg}}=\frac{\tau_{0}+\tau_{1}+\tau_{2}}{3}
\end{aligned}
$$

The lift and drag on the triangular clement (including both pressure and viscous effects) are then calculated by

$$
\begin{aligned}
& L=p_{\text {avg }} A_{w} \frac{C_{y}}{|\vec{C}|}+\tau_{\text {avg }} A_{w} \frac{B_{y}}{|\vec{B}|} \\
& D=p_{\text {avg }} A_{w} \frac{C_{z}}{|\vec{C}|}+\tau_{\text {avg }} A_{w} \frac{B_{x}}{|\vec{B}|}
\end{aligned}
$$




\subsubsection{Centrifugal Lift}

The high speeds at which hypersonic aircraft travel make the centrifugal lift term non-negligible. In fact, at Mach 12 the centrifugal lift can account for approximately $15 \%$ of the total lift on the vehicle. The centrifugal lift is calculated by

$$
L_{\text {cent }}=m_{\mathrm{veh}} \frac{V^{2}}{R_{e}+H}
$$

where $L_{\text {cent }}$ is the centrifugal lift, $V$ is the velocity of the vehicle, $R_{e}$ is the radius of the Earth and $H$ is the altitude of flight.

\subsubsection{Trajectory Model}

Knowledge of flowfield properties over the entire vehicle for a range of Mach numbers and angles of attack allows for performance calculations of the vehicle as a whole along a flight trajectory. The trajectory model for a nonrotating spherical Earth with the range in the axial $(\mathrm{x})$ direction as the dependent variable ${ }^{53}$ is

$$
\begin{gathered}
\frac{d H}{d r}=\tan \gamma_{f}\left(1+\frac{H}{R_{e}}\right) \\
\frac{d \gamma_{f}}{d r}=\left(\frac{L-m g \cos \gamma_{f}}{m V^{2} \cos \gamma_{f}}+\frac{1}{R_{e}+H}\right)\left(1+\frac{H}{R_{e}}\right) \\
\frac{d V}{d r}=\left(\frac{T h-D-m g \sin \gamma_{J}}{m V \cos \gamma_{f}}\right)\left(1+\frac{H}{R_{e}}\right) \\
\frac{d m}{d r}=-\frac{\dot{m}}{V \cos \gamma_{f}}\left(1+\frac{H}{R_{e}}\right)
\end{gathered}
$$

where $H$ is the altitude, $r$ is the range, $\gamma_{f}$ is the flight path angle, $R_{e}$ is the radius of the Earth, $L$ is the aerodynamic lift (does not include centrifugal lift), $m$ is 
the mass of the vehicle, $g$ is the gravitational acceloration, $T h$ is the thrust and $D$ is the drag. The performance is calculated by assuming a known trajectory profile ( $I$ vs. $r$ ). Knowing the trajectory profile allows for the solution of the flight path angle from Eq. 2.45. The flight path angle is a known function of tho range since the trajectory profile is given, hence the left hand side of Eq. 2.46 is known. The solution for the trajectory is then solved itcratively to find the angle of attack which makes the right hand sicle of Eq. 2.46 cqual to the left liand side. Once the angle of attack is known, the velocity and vehicke mass profile are then solved using Eqs. 2.47 and 2.48, respectively. 


\section{Chapter 3}

\section{RBCC Engine Model}

The RBCC ongine model is based boosely on descriptions in the open literature of Aerojet's Strutjet engine concept ${ }^{43}$ shown in Fig. 1.11. The ongine is composed of a set of strut injectors at the inlet of the engine, followed by a constiant area combustor section within the struts and ending with a constant angle expansion section. The injectors act as fuel-rich rockets at subsonic speeds which entrain incoming air from the inlet, thus raising the engine's specific impulse $I_{\mathrm{sp}}$ compared to rocket-only operation. At transonic-supersonic speeds, the struts act as isolators slowing the supersonic incoming air to sulsonic specels. In this speed regine, the engine transitions fron a fuel-rich rocket to a ramjet configuration. At high supersonic through bypersonic specds, the engine transitions from a ramjot to a scramjet. The struts act as normal fuel injectors, as well as isolators. The incoming flow is divided into smaller cross-sections, so that mixing of the fuet and the air is more efficient. Splitting the incoming flow also has the effect of reducing the length of the isolator section ${ }^{33}$.

The centerline geometry of the vehicle in scranjet mode is shown in Fig. 3.1. The forebody shock and the inlet ramp shocks all meet at the engine cowl at on-design conditions. The reffected shock off the engine cowl cancels out at the top of the enginc, where the shock/wall interaction is considered inviscid. The 


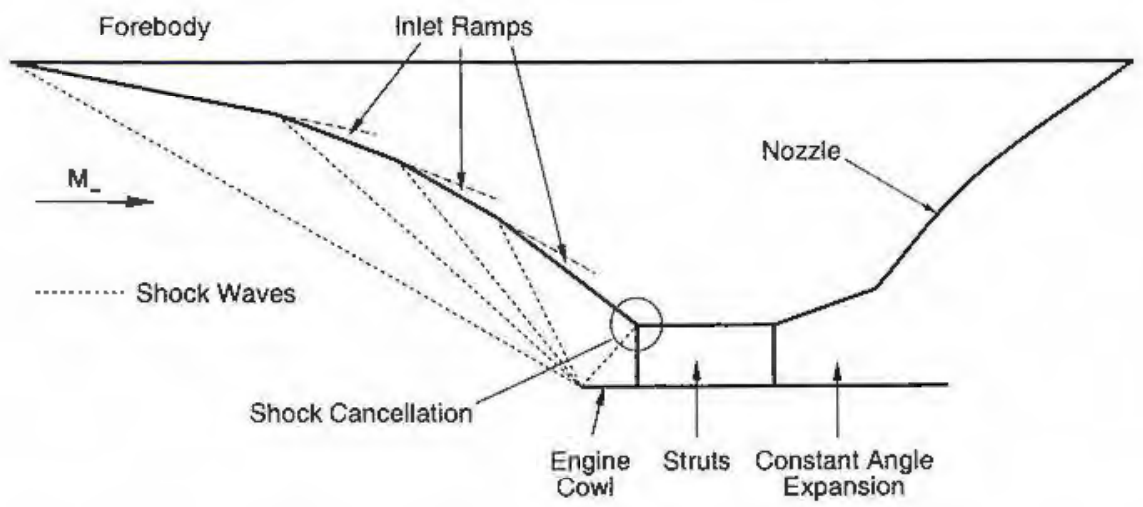

Figure 3.1: Vehicle centerline geometry in scramjet mode (not to scale).

RBCC struts are located immediately following the shock cancellation region and are contained entirely within the combustor. Following the struts, a constant angle expansion section leads to the internal and external nozzle sections.

In this work, only the airbreathing constituents of the RBCC engine (ramjet and scramjet) are modeled. However, problems with inlet starting are not considered, so the transition from ramjet to scramjet is modeled as instantaneous. The ducted rocket mode at low-speed and the pure rocket mode at high speed are not considered. In the following sections, the methodology to determine the engine flowfield characteristics for the airbreathing portion of the flight trajectory will be developed.

\subsection{Supersonic Strut Interaction Model}

The analytical, inviscid, supersonic strut inlet model consists of a calculation of the interaction between two struts. The side walls of the combustor are assumed to be half an injector each to simplify calculations. The flowfield is assumed to be fully two-dimensional (no flow spillage) and inviscid, thus shock wave/boundary layer interactions (which may be quite significant) are neglected. 


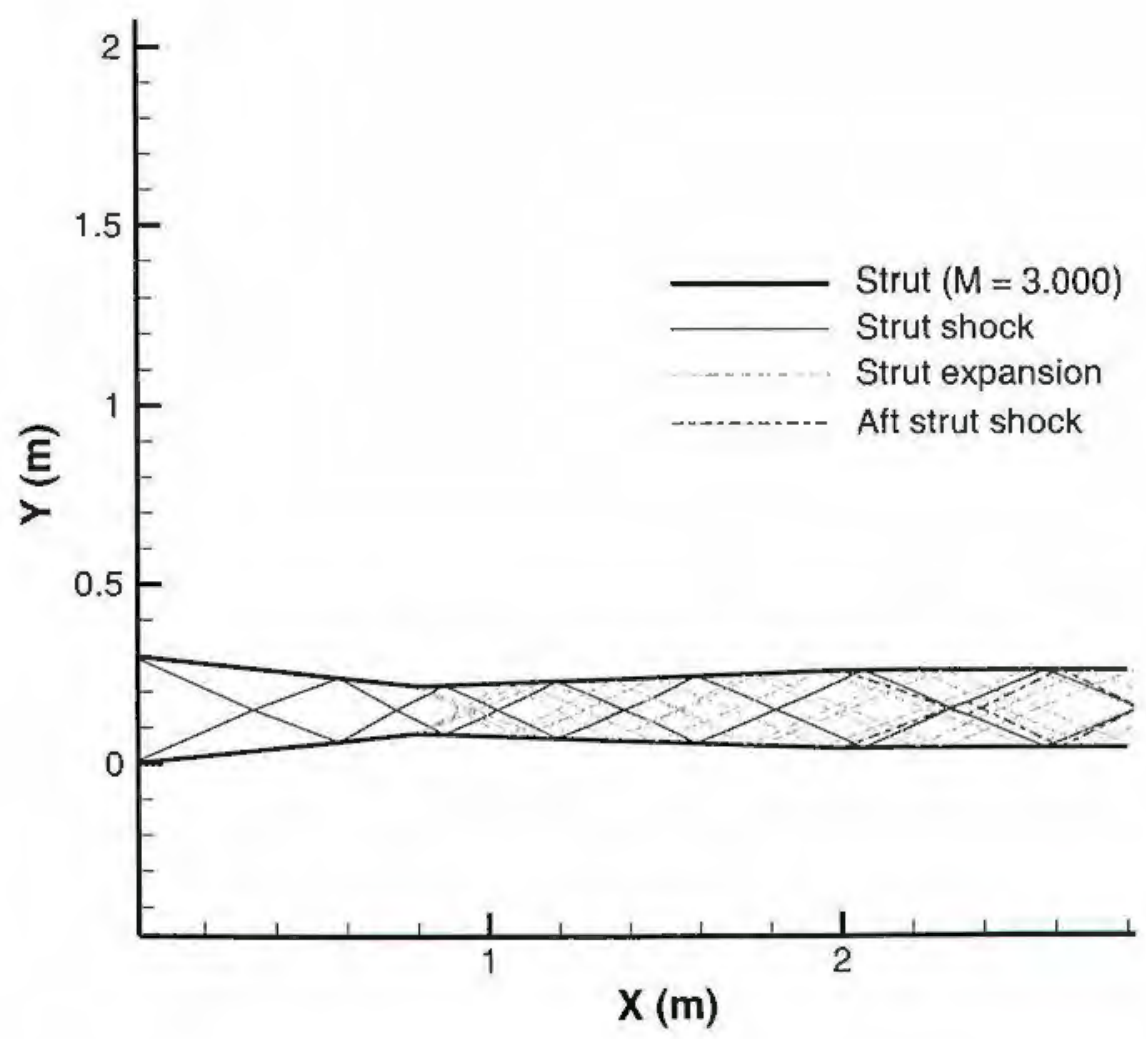

Figure 3.2: Analytical solution of strut/strut interaction $(M=3)$.

An analytical solution to the supersonic strut/strut interaction is shown in Fig. 3.2. The solution is calculated for an incoming Mach number of 3. Shock properties are calculated using the oblique shock relations. The continuous expansion fans are modeled as three discontinuous expansion waves, where properties across each wave are calculated using the Prandtl-Meyer relation.

Three types of wave interaction are identified: interaction of waves of opposite family, interaction of waves of the same family, and wave/wall interactions. Opposite family interactions involve waves that have angles of incidence that are opposite in sign. Same family interactions involve waves that have angles of incidence that are the same in sign. Wave/wall interactions involve shock waves or expansion waves reflecting off the strut walls. Three wave interactions are calculated for each family: shock/shock, shock/expansion, and expansion/expansion. 
The three types of wave interactions of opposite family are shown in Fig. 3.3. Referring to Fig. 3.3, the direction of positive angle is in the counterclockwise direction from the horizontal ( $\theta_{b}$ is positive, $\theta_{a}$ is negative). For each interaction, the necessary conditions behind the waves are that the pressure and flow direction are equivalent. Thus, to find these conditions, the oblique shock and Prandtl-Meyer relations are iterated to find the shock and/or expansion wave angles which produce the necessary conditions.

The three types of wave interactions of same family are shown in Fig. 3.4. The same nomenclature for positive angle direction discussed above is used for these interactions as well. Again, the oblique shock and Prandtl-Meyer relations are iterated such that the flow following the waves has constant pressure and the same direction. For these interactions, waves of opposite sign usually result. For shock/shock and shock/expansion interactions, the resulting waves of opposite sign are a shock and an expansion wave. For expansion/expansion interactions, two expansion waves result. For all wave/wave interactions, even though the pressure and direction must be equivalent, the temperature and Mach number need not be. Thus, for some interactions a small difference in temperature and Mach number occurs along the slip line. These differences are averaged in the analysis so that the flow following the wave/wave interactions have constant pressure, temperature, and Mach number.

The two wave/wall interactions are shown in Fig. 3.5. For the inviscid shock/wall interaction (shown in Fig. 3.5(a)), the flow direction prior to shock/wall intersection is traveling in the direction of the wall $\theta_{w}$. Behind the shock, the flow is traveling at an angle of $\theta_{a}$. The shock reflection is the same as the flow behind the leading shock being turned an angle of $\theta_{a}-\theta_{w}$. Using the oblique shock relations for the conditions ahead of the reflected shock, the shock properties behind the reflected shock are found. The reflected shock wave angle with respect to horizontal is then $\beta_{a}-\theta_{a}$. In the same fashion, the expansion/wall 


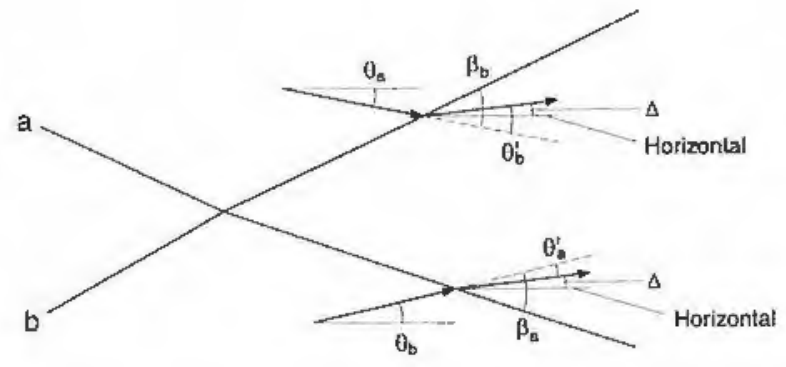

(a) Shock/Shock

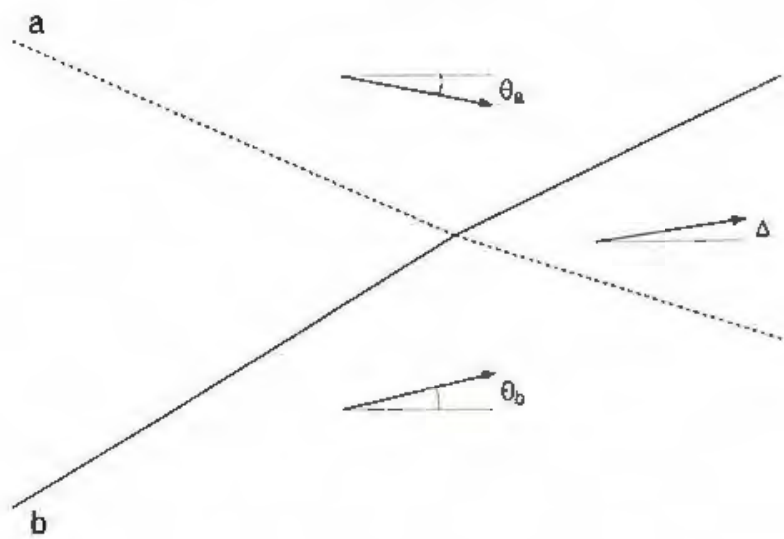

(b) Shock/Expansion

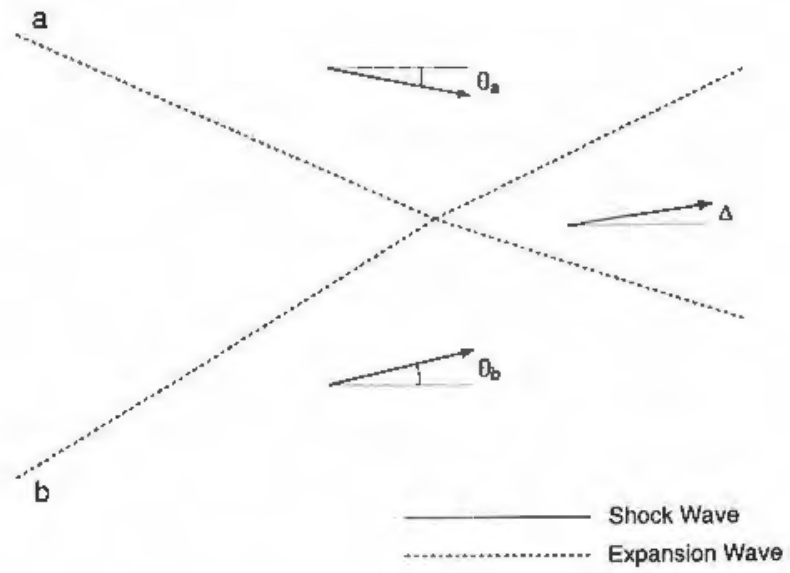

(c) Expansion/Expansion

Figure 3.3: Wave interactions of opposite family. 


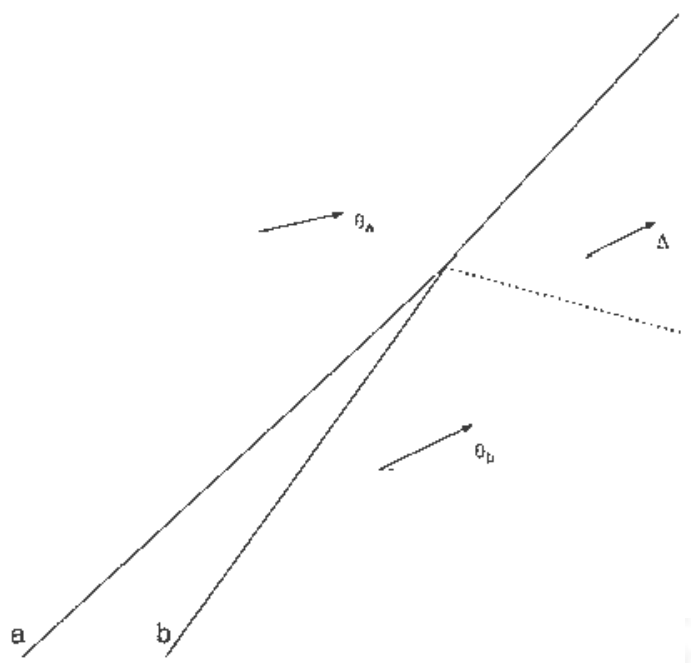

(a) Shook/Shork

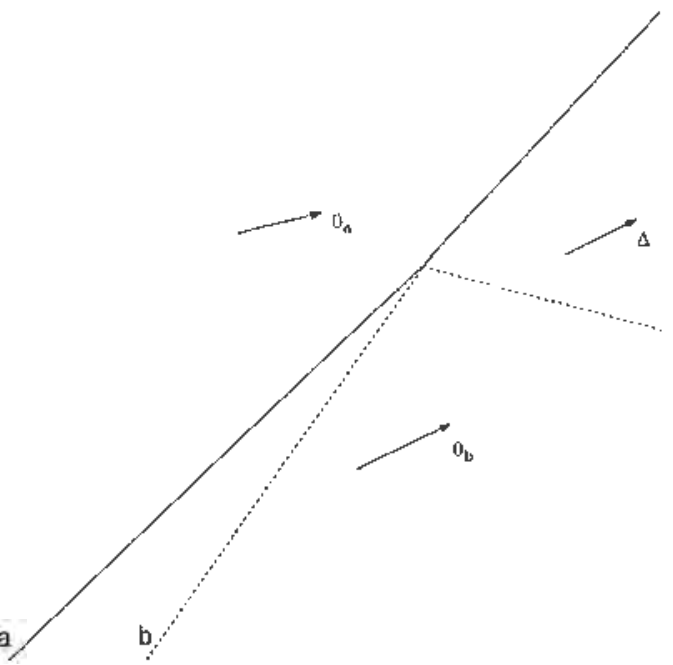

(b) Shock/Expansion

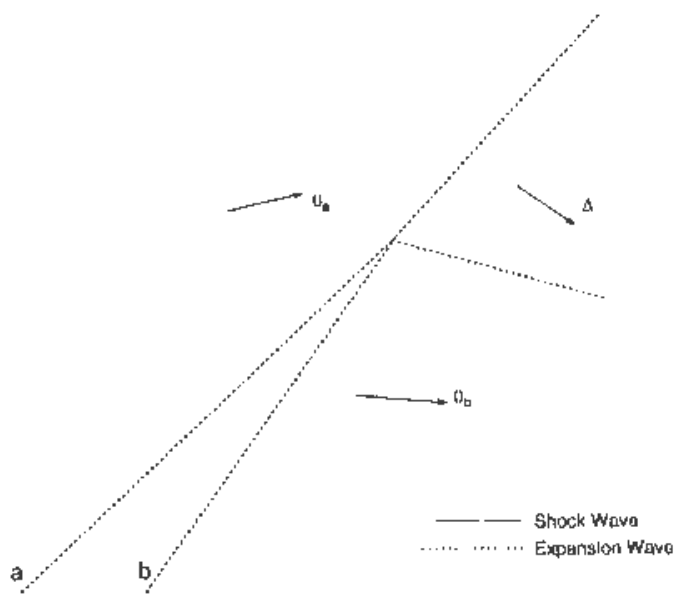

(c) Expansion/Expuntsion

Figure 3.4: Wave interactions of same family. 


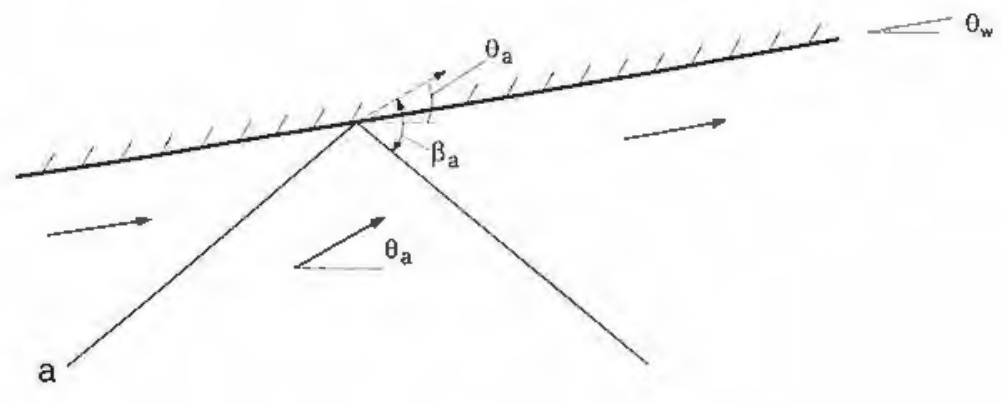

(a) Shock/Wall

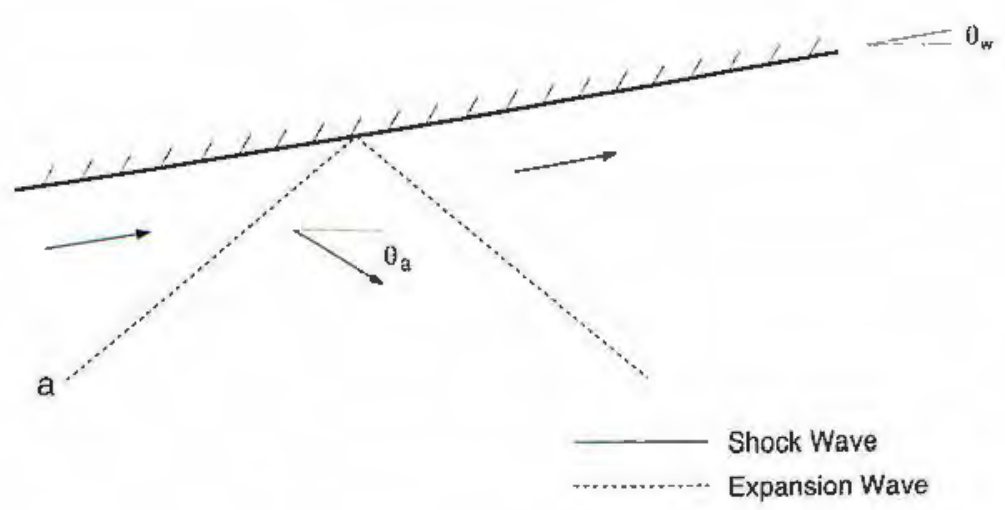

(b) Expansion/Wall

Figure 3.5: Wave/wall interactions.

interaction may be calculated by tracking the flow direction before and after the wall using the Prandtl-Meyer relation.

\subsubsection{Validation}

The analytical model was validated by solving the two-dimensional flowfield using the General Aerodynamic Simulation Program (GASP) v3.0 ${ }^{54}$. GASP solves the integral form of the unsteady Reynolds-Averaged Navier-Stokes (RANS) equations in three dimensions subject to boundary and initial conditions. For two-dimensional cornputation the user defines a two-dimensional grid that is one 


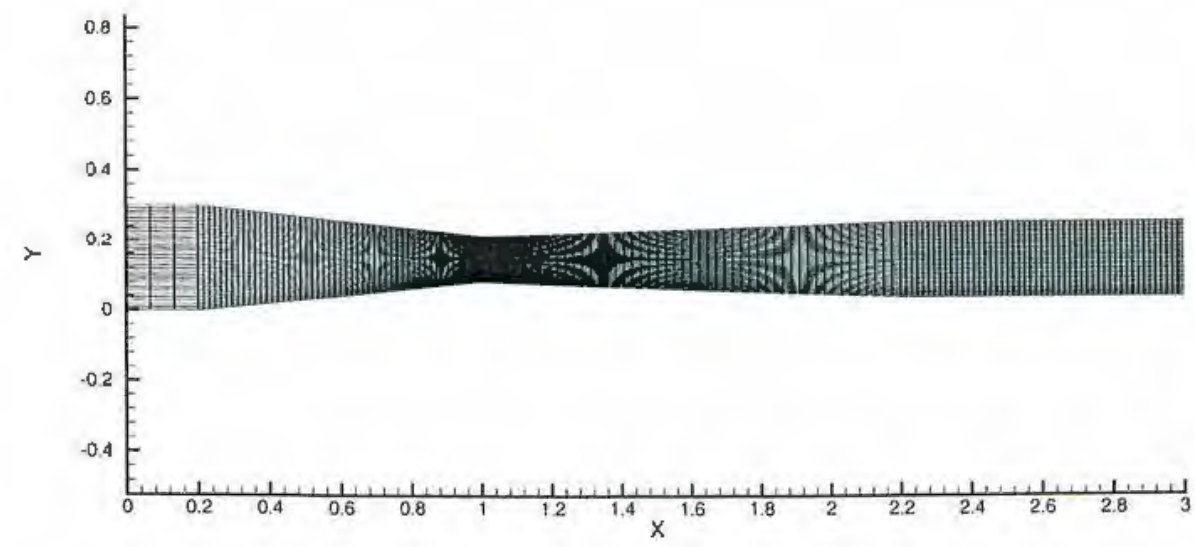

Figure 3.6: Computational grid used for strut/strut flowfield validation.

cell thick. The inviscid, steady flowfield is calculated from the Euler equations (a subset of RANS) with no chemical reactions and space marching in the axial direction.

The grid dimensions for the validation runs had 460 points in the flow direction and 100 points in the cross-flow direction (see Fig. 3.6, shown is one out of every three grid points). A computational solution for pressure is plotted in Fig. 3.7 for an incoming Mach number of 3. Superimposed on the computational solution is the analytical solution for the strut shocks (expansion waves and aft strut shocks are eliminated for clarity). Good agreement is found between the analytical and the computational solution for shock shape in the regions where shock strength is relatively high. As the shock dissipates further downstream (due to flow expansion as well as grid resolution), the analytical prediction for shock location deviates. Discrepancies between the analytical solution and the computational solution are primarily due to the finite discretization of the expansion fan and are partially due to grid resolution in the region where the strut shocks first interact with the expansion fans.

The computational results for area averaged exit pressure are compared to the 


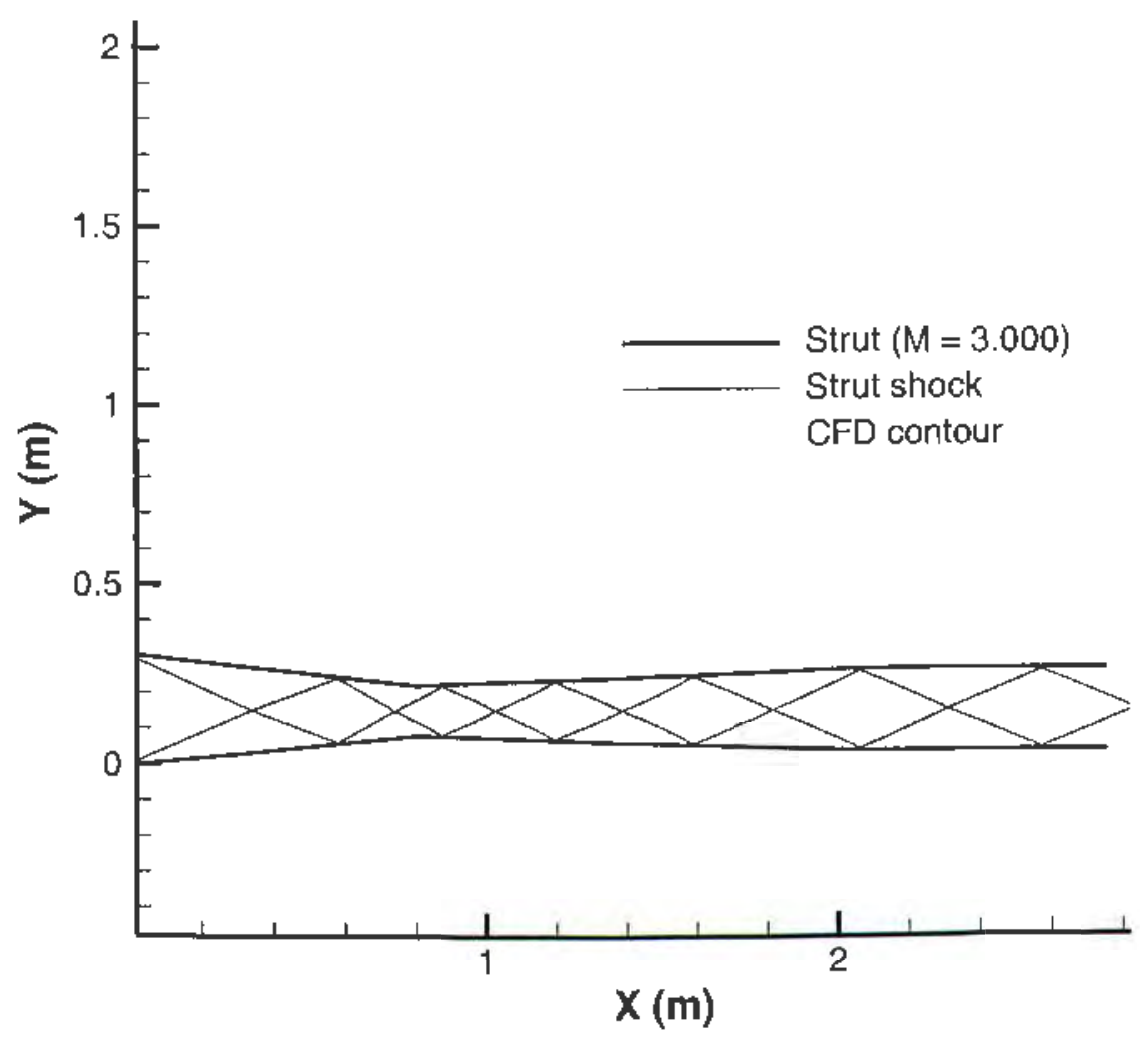

Figure 3.7: Analytical solution to strut/strut interaction superimposed upon pressure contours of CFD Solntion ( $\mathrm{M}=3)$.

anatytical model for the strut-strut interaction in Fig. 3.8. The same geomotry is solved for an incoming pressurc of $1 \mathrm{~atm}$. and a wide range of inlet Mach numbers. Also ploted in Fig. 3.8 is the prodiction using inviscid, quasi-1D fow analysis. The analytical model correctly predicted the pressure vs. Mach number trend found from the computational solution, but slightly overpredicts the pressure for all Mach numbers. The maximum crror in pressure overprediction was found to be approximately $3 \%$. This error can be attributed to the limited number of discontinuous waves discretizing the continuous expansion fan. The error in prossure was deemed acceptable considering the additional computational costs involved in further discretizing the expansion fan, and errors inherent to the model for neglecting shock wave / boundary layer interactions. Also, the trends 


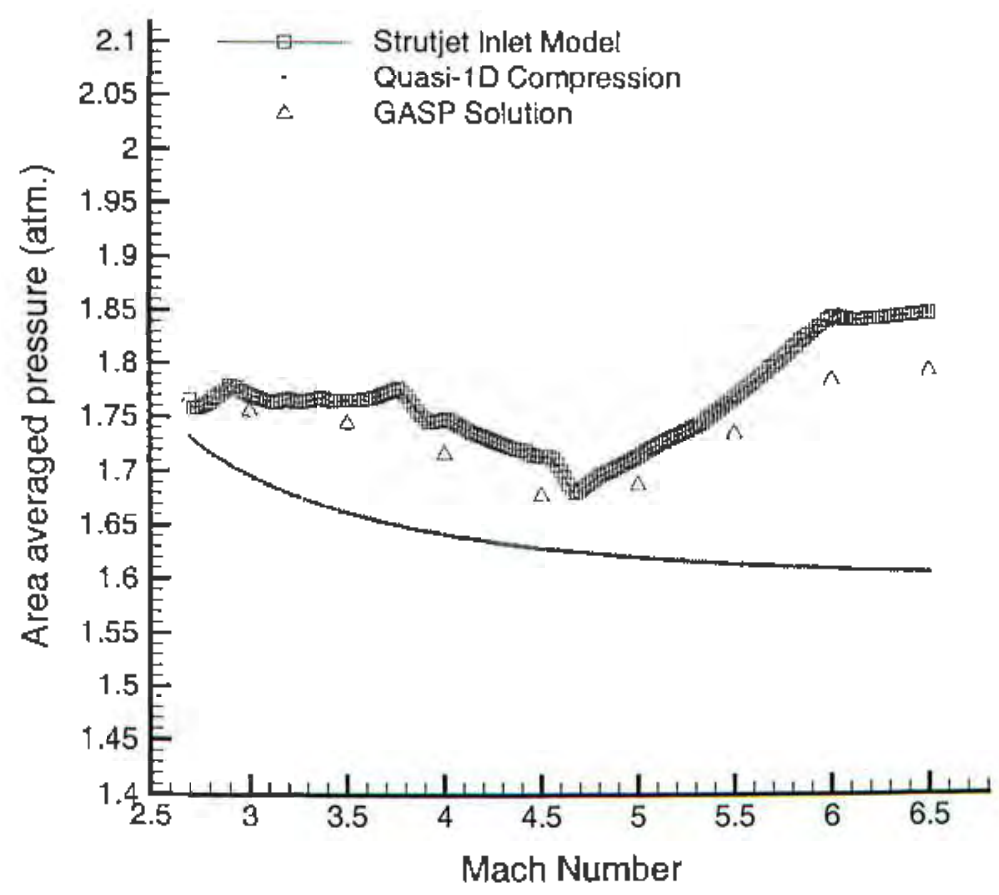

Figure 3.8: Area averaged exit pressure vs. Mach number for strut/strut interaction.

in Fig, 3.8 sllow that inviscid, quasi-1D flow does not correctly predict both the qualitative and quantitative behavior of the strut/strut interaction.

\subsection{Supersonic Combustion Flowfield Model}

The governing equations for the engine norlel ${ }^{55}$ are based on the following assumptions: 1) quasi-one-dimensional fow, where all variables (including area) are functions in the $x$-direction only, 2) stcady-state flow, and 3) perfect gas. 


\subsubsection{Continuity}

The quasi-one-dimensional, steady continuity equation may be stated as

$$
\dot{m}=\rho U A
$$

where $\dot{m}$ is the mass flow rate, $\rho$ is the density of the flowfield, $U$ is the velocity of the flowfield, and $A$ is the cross-sectional area of the flowfield. The continuity equation (Eq. 3.1) may be expressed in differential form ${ }^{36}$ as

$$
\frac{1}{\dot{m}} \frac{d \dot{m}}{d x}=\frac{1}{\rho} \frac{d \rho}{d x}+\frac{1}{U} \frac{d U}{d x}+\frac{1}{A} \frac{d A}{d x}
$$

The differential continuity equation (Eq. 3.2) allows for variation in cross-sectional area $(d A / d x)$ of the combustor (geometry effects) and mass injection $(d \dot{m} / d x)$ of fuel, pilot, or other diluent.

Any mass that is injected from a discrete location is assumed to follow a userprescribed mass mixing profile. The mass injected will take a finite amount of time to be added to the system, to completely affect the freestream in a quasione-dimensional sense. Once the mass is added to the system, it is assumed to be instantaneously mixed with the engine flowfield. Mass injected from an injection port is added to the flowfield incrementally in the $x$-direction by the mass addition term $\frac{d \dot{m}}{d x}$. In this manner, mass is added to the flow as it travels through the engine until the full amount of mass injected is present. This mass addition term should not be confused with fuel mixing. Although mass and fuel mixing may be treated as the same mechanism, they are not inherently the same. The mass addition term is solely used for telling the engine flowfield that more mass has been added. Fuel mixing is prescribed to tell the flowfield when the injected fuel is available for reaction (i.e., enough mixing has occurred between the air and fuel such that combustion may take place). 


\subsubsection{Momentum}

The quasi-one-dimensional momentum equation in differential form ${ }^{53}$ may be expressed as

$$
\frac{1}{p} \frac{d p}{d x}+\frac{\gamma M^{2}}{2 U^{2}} \frac{d U^{2}}{d x}+\frac{2 \gamma M^{2} C_{f}}{\mathcal{D}}+\frac{\gamma M^{2}(1-\varepsilon)}{\dot{m}} \frac{d \dot{m}}{d x}=0
$$

where $p$ is the pressure, $\gamma$ is the ratio of specific heats, $M$ is the Mach number, $C_{f}$ is the friction cocficient, $\mathcal{D}$ is the hydraulic diameter (Eq. 2.34), and $\varepsilon$ is the ratio of the velocity of gas injection in the $\mathrm{x}$-direction over the velocity of the flowfield

$$
\varepsilon \equiv \frac{U_{i}}{U_{\infty}}
$$

Equation 3.4 accounts for angled fuel injection ( $\varepsilon=0$ - normal fucl injection). The momentum equation (Eq. 3.3) accounts for viscosity in the flow as a momentum deficit. The friction coefficient may be found from experimental results or by some analytical or empirical method. The friction coefficient used in the validation and application problems to follow is calculated using Eckert's refercnce temperature method ${ }^{51}$ assuming fully turbulent flow. The viscosity is evaluated at the reforence temperature by using Sutherland's Law.

\subsubsection{Equation of State}

Assuming a perfect gas, the equation of state may be expressed as

$$
p=\frac{\rho R_{u s} T}{\overline{M W}}
$$

where $R_{u}$ is the universal gas constant and $\overline{M W}$ is the mixture molecular weight. Fxpressing the equation of state in differential form ${ }^{57}$ yields

$$
\frac{1}{p} \frac{d p}{d x}=\frac{1}{\rho} \frac{d \rho}{d x}+\frac{1}{T} \frac{d T}{d x}-\frac{1}{\overline{M W}} \frac{d \overline{M W}}{d x}
$$

The abowe form for the equation of state allows for the ehemical changes due to mass injection and combustion to be accounted. 


\subsubsection{Mixture Molecular Weight}

The mixture molecular weight may be cxpressed in terms of the mass fraction as

$$
\overline{M W}=\frac{1}{\sum_{i} \frac{Y_{i}}{M W_{i}}}
$$

where $Y_{i}$ is the mass fraction of species $i$ and $M W_{i}$ is the molecular woight of species $i$. The differmtial form of the mixture molecular weight has the form 57

$$
\frac{d \overline{M W}}{d x}=-\overline{M W}^{2}\left(\sum_{i} \frac{1}{M W_{i}} \frac{d Y_{i}}{d x}\right)
$$

\subsubsection{Species Conservation}

The derivation of the species conservation equation is based on derivations given by Turns ${ }^{57}$. The finite control volume for the quasi-one-dimensional, steady case is shown in Fig. 3.9. Conservation of species $i$ in Fig. 3.9 is found to be

$$
\left[\dot{m}_{i}^{\prime \prime} A\right]_{: i+\Delta x}-\left[\dot{m}_{i}^{\prime \prime} A\right]_{x}=\dot{m}_{i}^{\prime \prime \prime} V+\dot{m}_{i, \text { add ded }}
$$

where $\dot{m}^{\prime \prime}$ is the mass fow rate per unit area, $\dot{m}^{\prime \prime \prime}$ is the mass flow rate per unit volume, $V$ is the volume, and subscript added denotes the addition of species $i$ at a particular location of $x$ due to mass addition. The left hand side of Eq. 3.9 is seen as the net mass flow of species $i$ leaving the control volume. The first term on the right hand side of Eq. 3.9 is a source or sink term due to chemical reaction within the control volume. The sccond term on the right hand side is a source term that models mass/fuel injection.

Neglecting molecular diffusion in the flow direction, Eq. 3.9 may be expressed as

$$
\left[Y_{i} \dot{m}^{\prime \prime} A\right]_{x+\Delta x}-\left[Y_{i} \dot{m}^{\prime \prime} A\right]_{x}=\dot{m}_{i}^{\prime \prime \prime} A \Delta x+\dot{m}_{i, \text { adderl }}
$$




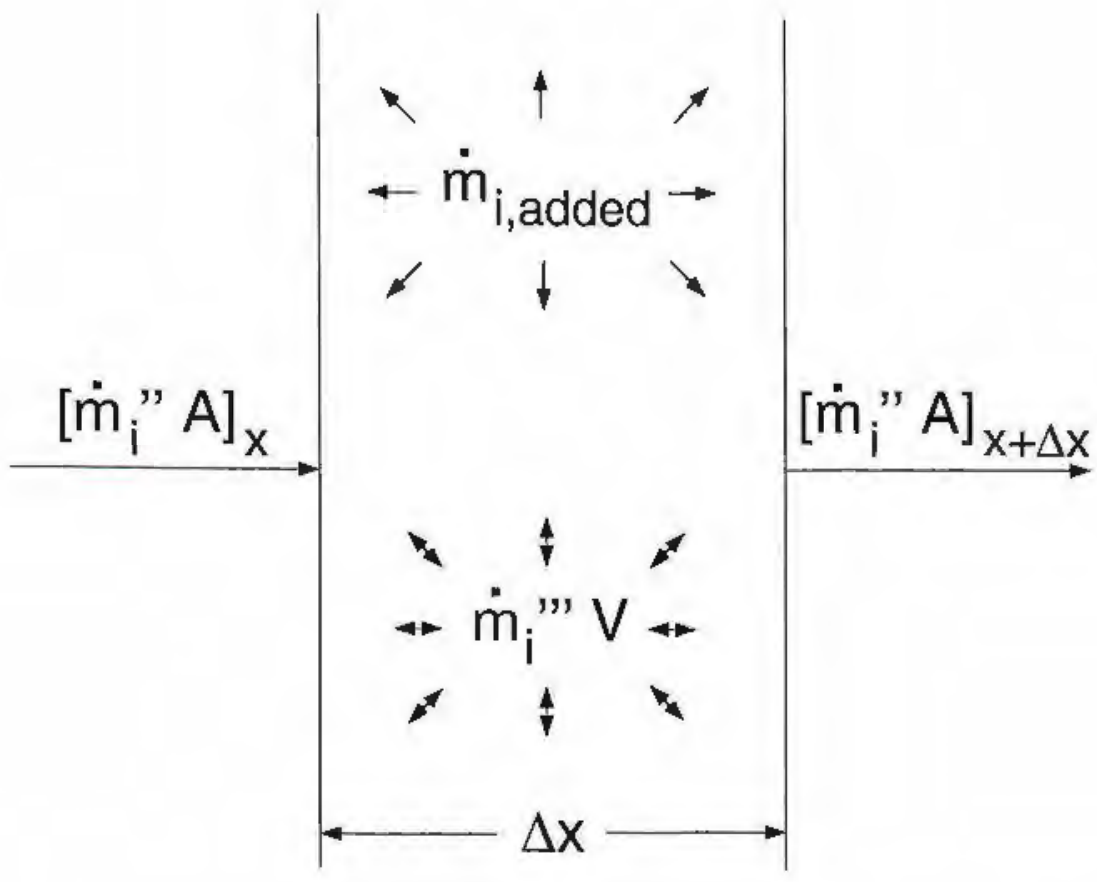

Figure 3.9: Quasi-one-dimensional control volume for species conservation derivation.

where the volume of the quasi-one-dimensional control volume is $A \Delta x$. As $\Delta x$ becomes infinitesimally small, Eq. 3.10 may be expressed in differential form as

$$
\frac{1}{A} \frac{d\left[Y_{i} \dot{m}^{\prime \prime} A\right]}{d x}=\dot{m}_{i}^{\prime \prime \prime}+\frac{1}{A} \frac{d \dot{m}_{i, \text { added }}}{d x}
$$

The source term $\dot{m}_{i}^{\prime \prime \prime}$ may be expressed as

$$
\dot{m}_{i}^{\prime \prime \prime}=\dot{\omega}_{i} M W_{i}
$$

where $\dot{\omega}_{i}$ is the molar production rate of species $i$ from chemical reactions. Separating the mass fraction from the differential in Eq. 3.11 and substituting Eq. 3.12 yields the differential species conservation equation

$$
\frac{d Y_{i}}{d x}=\frac{\dot{\omega}_{i, \operatorname{mix}} M W_{i}}{\rho U}+\frac{1}{\dot{m}} \frac{d \dot{m}_{i, \text { added }}}{d x}-\frac{Y_{i}}{\dot{m}} \frac{d \dot{m}}{d x}
$$

Equation 3.13 states that the change in mass fraction of species $i$ is a function 
of the rate of production/destruction of species $i$, the amount of species $i$ axlded, and the total amount of mass adcled.

It is noterl that the subscript "mix" has been added to the production rate in Eq. 3.13 to denote fuel mixing. In autdition to the proscribed mass addition profile $\frac{d r n}{d x}$, a fuel mixing profile may be prescribed to nodel when the fuel that has been injected is awailable for reaction. This mixing profile may take the form of a mixing efficiency (where a certain percentage of fuel injected is never available for reaction) and/or a mixing length ${ }^{55}$ (where after the fuel has been added to the flow in a mass sense, a finite amount of mixing time is necessary before reaction may begin to occur). In this study, only a mixing officiency will be used. The mixing efficiency is prescribed to model unmixed fucl in the combustor but can also represent inefficiencies and losses due to fucl injection.

\subsubsection{Energy}

The derivation of the energy equation is partially based on derivations presented by Turns ${ }^{n 7}$. The finite control volume for the quasi-one-dimensional, stearly energy equation derivation is shown in Fig. 3.10. Neglecting axial heat. conduction, axial species diffusion, radiation, and work done on the control volume, conservation of energy in Fig. 3.10 yields the following equation

$$
\begin{gathered}
\left.\left[\dot{m}^{\prime \prime} A h_{o}\right]_{x+\Delta x}-\left[\dot{m}^{\prime \prime} A h_{v}\right]_{x}=\left[\sum_{i} h_{i} \dot{m}\right]_{i}\right]_{\text {added }}-\dot{Q}^{\prime \prime} P_{w} \Delta x \\
h_{o} \equiv h+\frac{U^{2}}{2}
\end{gathered}
$$

where $h_{o}$ is the total or stagnation enthalpy per unit mass and $\dot{Q}^{\prime \prime}$ is the local heat transfer rate per unit area. The left hand side of Eq. 3.14 is the net anound, of energy flow leaving the control wolume. The first term on the right hand side of Eq. 3.14 is a source term for enthalpy addition due to fucl injection, and 


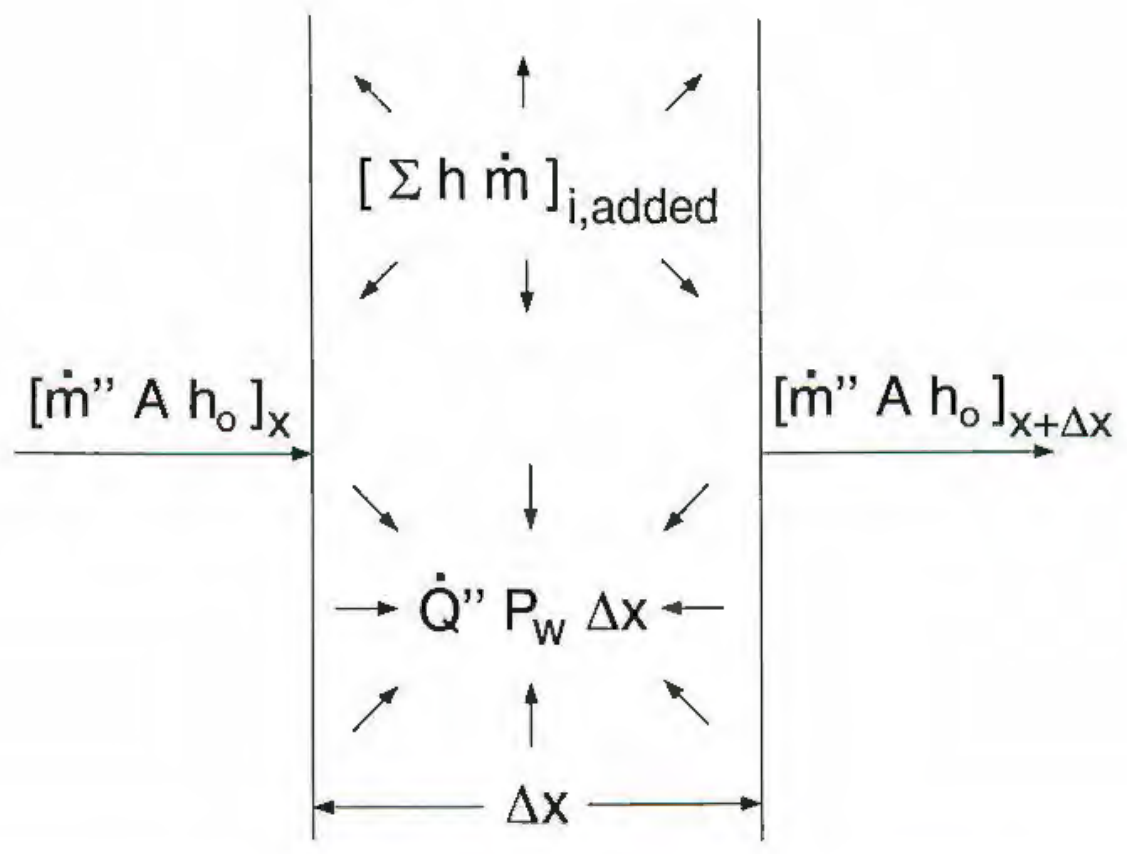

Figure 3.10: Quasi-one-dimensional control volume for energy derivation.

assumes thermal equilibrium between the fuel and the flowfield. The second term on the right hand side of Eq. 3.14 is a sink term for heat transferred to the engine wall. As $\Delta x$ becomes infinitesimally small, the energy equation becomes

$$
\frac{d\left[\dot{m}^{\prime \prime} A h_{o}\right]}{d x}=\frac{d\left[\sum_{i} h_{i} \dot{m}_{i}\right]_{\text {added }}}{d x}-\dot{Q}^{\prime \prime} P_{w}
$$

Using the chain rule, Eq. 3.16 is rewritten as

$$
\frac{d h}{d x}=\frac{1}{\dot{m}} \frac{d\left[\sum_{i} h_{i} \dot{m}_{i}\right]_{\mathrm{added}}}{d x}-\frac{\dot{Q}^{\prime \prime} P_{w}}{\dot{m}^{\prime \prime} A}-\frac{h_{o}}{\dot{m}} \frac{d \dot{m}}{d x}-U \frac{d U}{d x}
$$

The enthalpy of the flowfield may be written in terms of the enthalpy of each species

$$
h=\sum_{i} c_{p_{i}} T Y_{i}
$$

where the flowfield is assumed to be thermally perfect so that enthalpy is not a function of flowfield pressure. The derivative of enthalpy as a function of axial 
distance may then be found from Eq. 3.18

$$
\frac{d h}{d x}=\sum_{i}\left[h_{i} \frac{d Y_{i}}{d x}+T Y_{i} \frac{d c_{p_{i}}}{d x}\right]+c_{p} \frac{d T}{d x}
$$

The specific heat of species $i$ may be represented by a curvefit with respect to tomperature in the following form

$$
c_{p_{i}}=\frac{R_{u}}{M W_{i}}\left(a_{1 i}+a_{2 i} T+a_{3 i} T^{2}+a_{4 i} T^{3}+a_{5 i} T^{4}\right)
$$

where $R_{u}$ is the universal gas constant and the coefficionts $a_{1 i}-a_{5 i}$ are curvefit constants for specios $i$. Equation 3.20 is in the form used by the NASA chemical equilibrium codes". Assuming the constants $a_{1 i}-a_{5 i}$ are weak functions of temperature, the derivative of $c_{p_{i}}$ with respect to axial distance yields an equation in terms of temporature derivative

$$
\begin{gathered}
\frac{d c_{p_{i}}}{d x}=\frac{R_{u s}}{M W_{i}}\left(a_{2 i}+2 a_{3 i} T+3 a_{4 i} T^{2}+4 a_{5 i} T^{3}\right) \frac{d T}{d x}=\vec{c}_{p_{i}} \frac{d T}{d x} \\
\bar{c}_{p_{i}} \equiv \frac{R_{u}}{M W_{i}}\left(a_{2 i}+2 a_{3 i} T+3 a_{4 i} T^{2}+4 a_{5 i} T^{3}\right)
\end{gathered}
$$

Substituting Eq. 3.21 into Eq. 3.19 yiclds the hinal form of the derivative of enthalpy

$$
\begin{gathered}
\frac{d h}{d x}=\tilde{c}_{p} \frac{d T}{d x}+\sum_{i} h_{i} \frac{d Y_{i}}{d x_{i}} \\
\tilde{c}_{p} \equiv c_{p}+\sum_{i} T \tilde{c}_{p_{i}} Y_{i}
\end{gathered}
$$

In a similar manner, the derivative of enthalpy of species $i$ may be writton as

$$
\frac{d h_{p_{i}}}{d x}=\left(c_{p_{i}}+\tilde{c}_{p_{i}} T\right) \frac{d T}{d x}
$$

The heat transfer tem (second term on the right hand side of Eq. 3.17) is calculated from the definition of the Stanton number

$$
C_{I} \equiv \frac{\dot{Q}^{\prime \prime}}{\rho U\left(h_{\mathrm{aw}}-h_{w}\right)}
$$


where $C_{1 l}$ is the Stanton number and subseript aw denotes adiabatic wall conditions. The Stanton number may be related to the friction coeficient by Reynolds analogy

$$
C_{I I}=\frac{C_{f}}{2 \operatorname{Pr}^{2 / 3}}
$$

where $\mathrm{Pr}$ is the Prandtl number (assumed to be a constant value of 0.71 ). Substituting Eq. 3.27 into Eq. 3.26, the sccond term in Eq. 3.17 may be written as

$$
\frac{\dot{Q}^{\prime \prime} P_{w}}{\dot{m}^{\prime \prime} A}=\frac{2 C_{f} c_{p}\left(T_{\mathrm{aw}}-T_{w}\right)}{\operatorname{Pr}^{2 / 3} \mathcal{D} A}
$$

It is assumed that the wall enthalpies may be written as a function of frecstream specific heat at constant pressure and wall and adiabatic wall temperatures, where adiabatic wall temperature may be calculated from ${ }^{50}$

$$
T_{\mathrm{aw}}=T\left[1+\left(\operatorname{Pr}^{*}\right)^{1 / 3} \frac{\gamma-1}{2} M^{2}\right]
$$

where $P r^{*}$ is the Prandtl number evaluated at the reference temperature. For the current study, the reference Prandtl number is also assuned to have a value of 0.71 . Finaliy, substituting Eqs. $3.23,3.25$ and 3.28 into Eq. 3.17 yiclds the quasi-onc-dimensional, steady, differential energy equation

$$
\begin{aligned}
& \frac{d T}{d x}=\frac{1}{\hat{c}_{p}} {\left[-\sum_{i} h_{i} \frac{d Y_{i}}{d x}+\frac{1}{\dot{m}} \sum_{i}\left(h_{i} \frac{d \dot{m}_{i}}{d x}\right)_{\text {addet } d}\right.} \\
&\left.-\frac{2 C_{i} c_{p}\left(T_{\mathrm{aw}}-T_{w}\right)}{\operatorname{Pr}^{2 / 3} \mathcal{D} A}-\frac{h_{o}}{\dot{m}} \frac{d \dot{m}}{d x}-U \frac{d U}{d x}\right] \\
& \hat{c}_{p} \equiv \tilde{c}_{p}-\frac{1}{\dot{m}}\left\{\sum_{i}\left[\dot{m}_{i}\left(c_{p_{i}}+\tilde{c}_{p_{i}} T\right)\right]_{\mathrm{addlect}}\right\}
\end{aligned}
$$

\subsubsection{Solution Methodology}

Equations $3.2,3.3,3.6,3.8,3.13$ and 3.30 constitute a stiff set of ordinary differential equations (ODEs) due to the chemical production toms from com- 
bustion. Solution of these equations requires a stiff ODE solver which can account for differing time scales. A code named VODPK ${ }^{60}$, developed by Lawrence Livermore National Lab, was used to accomplish this task. VODPK uses a backward differentiation formula to integrate the set of stiff ODEs. Values for the individual chemical species molecular weight, specific heat, heat of formation, and reaction rates are supplied by CHEMKIN-II ${ }^{48}$ for a user supplied reaction mechanism. The ambient air composition is assumed to be $78 \%$ nitrogen, $21 \%$ oxygen, and $1 \%$ argon by volume. The hydrogen/air reaction mechanism used in this study was proposed by Jachimowski ${ }^{61}$ and is listed in Appendix B.

The mixing model used for perpendicular injection of hydrogen is a curvefit of data originally reported by Rogers ${ }^{62}$ and tabulated by Henry and Anderson ${ }^{63}$

$$
\begin{gathered}
\dot{m}_{r}=\dot{m}_{f} \frac{a \bar{x}^{b} \exp (c \bar{x})}{d \bar{x}+f} \\
\bar{x} \equiv \frac{x}{L_{\mathrm{mix}}}
\end{gathered}
$$

where $\dot{m}_{r}$ is the amount of mass ffow available for reaction, $\dot{m}_{f}$ is the total mass flow rate of fuel to be injected, and $L_{\text {mix }}$ is a mixing length. The curvefit constants have the values: $\mathrm{a}=1.1703, \mathrm{~b}=0.62925, \mathrm{c}=0.42632, \mathrm{~d}=1.4615$, and $\mathrm{f}=0.32655$. Taking the derivative of Eq. 3.32 yields

$$
\frac{d \dot{m}}{d x}=\frac{\dot{m}_{r}}{L_{\operatorname{mix}}}\left[\frac{c d \bar{x}^{2}+(b d+c f-d) \bar{x}+b f}{\bar{x}(d \bar{x}+f)}\right]
$$

where it is assumed for all scramjet cases that the mass mixing length is the same as the reaction mixing length.

Solving Eqs. 3.2, 3.3, 3.6 and 3.30 for the derivative in velocity yields

$$
\begin{array}{r}
\frac{d U}{d x}=\frac{1}{\alpha}\left\{-\frac{1}{A} \frac{d A}{d x}+\frac{1+\gamma M^{2}(1-\varepsilon)-\frac{h_{0}}{\hat{h}} \frac{d \dot{m}}{d x}-\frac{1}{\overline{M W}} \frac{d \overline{M W}}{d x}}{\dot{m}}+\frac{1}{\hat{h}}\left[-\sum_{i} h_{i} \frac{d Y_{i}}{d x}+\frac{1}{\dot{m}} \sum_{i}\left(h_{i} \frac{d \dot{m}_{i}}{d x}\right)_{\text {added }}\right]+\left[\gamma M^{2}-\frac{c_{p}\left(T_{\mathrm{aw}}-T_{w}\right)}{\hat{h} P r^{2 / 3} A}\right] \frac{2 C_{f}}{\mathcal{D}}\right\}
\end{array}
$$




$$
\begin{gathered}
\alpha \equiv \frac{1}{U}\left(1-\gamma M^{2}+\frac{U^{2}}{\hat{h}}\right) \\
\hat{h} \equiv \hat{c}_{p} T
\end{gathered}
$$

By examination, it will be shown that each term in Eq. 3.35 can be calculated or is a prescribed quantity. The first term in Eq. 3.35 is the assumed crosssectional area profile $\frac{d A}{d x}$. The second term is the assumed mass flow addition term $\frac{d \dot{m}}{d x}$ and represents the mixing profile. The quantity $\hat{h}$ can be calculated using CHEMKIN-II. Solution of the change in mixture molecular weight $\frac{d \overline{M W}}{d x}$ is found by first solving the species conservation equation (Eq. 3.13). Given the chemical information from CHEMKIN-II and the mixing profile, Eq. 3.13 is a known quantity which may then be substituted into Eq. 3.8 to solve for the change in mixture molecular weight. The terms involving the friction coefficient are all known quantities or may be calculated using CHEMKIN-II. The remaining terms in Eq. 3.35 are known quantities, quantities that have already been calculated, or quantities that may be calculated using CHEMKIN-II. Thus, the velocity derivative derived in Eq. 3.35 is a known quantity at a particular $x$-location.

With knowledge of the velocity derivative, the density derivative may be found from the continuity equation (Eq. 3.2). The pressure derivative may be calculated from the momentum equation (Eq. 3.3). The temperature derivative is then found from the equation of state (Eq. 3.6). The derivatives of all the variables are then integrated using VODPK to find the flow solution. The full engine flowfield may be calculated on the order of one to two seconds using a $433 \mathrm{MHz}$ DEC-ALPHA computer. Thus, this method allows for rapid design of full vehicle concepts that include a detailed engine flowfield. 


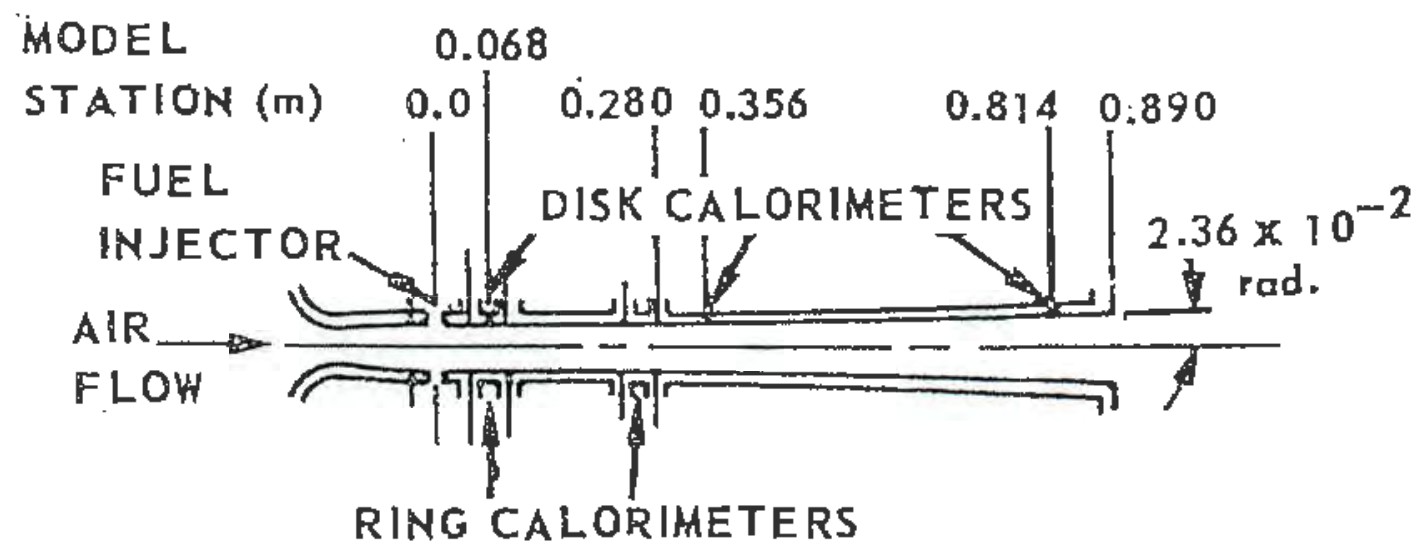

Figure 3.11: Experimental configuration for Ref. 64.

\subsubsection{Scramjet Mode Engine Validation}

The combustor model just presented is now compared to a pair of experimental investigations into hydrogen fueled scramjet engines. The first experiment is an axisymmetric, wall-injection scramjet investigated by Billigen, as shown in Fig. 3.11. The combustor consists of a constant-area circular cylinder $(0.0038$ $\mathrm{m}^{2}$ ) that is $0.28 \mathrm{~m}$ long followed by a conical expansion section that is $0.61 \mathrm{~m}$ long, resulting in an area ratio of 2. Eight fuel injectors of diameter $2.64 \mathrm{~mm}$ each are evenly distributed along the circunference at the $x=0$ location.

Reported incoming conditions into the combustor as are: total temperature of $2180 \mathrm{~K}$, total pressure of $3.13 \mathrm{MN} / \mathrm{m}^{2}$, Mach number of 3.2 , fuel temperature of $705 \mathrm{~K}$, and an equivalence ratio of 0.5 . Unfortunately, the chemical composition of the incoming air was not specifically given in Ref: 64 , hence the statice properties entering the combustor can take on a wide range of values ${ }^{34,65}$. Reported experimental uncertainties for incoming pressure rariged from 52090 to $55000 \mathrm{~N} / \mathrm{m}^{2}$, incoming temperature from 820 to $940 \mathrm{~K}$ and wall temperature from 450 to $550 \mathrm{~K}$. The incoming air composition is assumed to be $78 \% \mathrm{~N}_{2}, 21 \%$ $\mathrm{O}_{2}$, and $1 \% \mathrm{Ar}$ by volume as previonsly discussed. In this study, the assumed incoming properties into the engine are: $U=1849 \mathrm{~m} / \mathrm{s}, \mathrm{p}=52000 \mathrm{~N} / \mathrm{m}^{2}, \mathrm{~T}=$ 


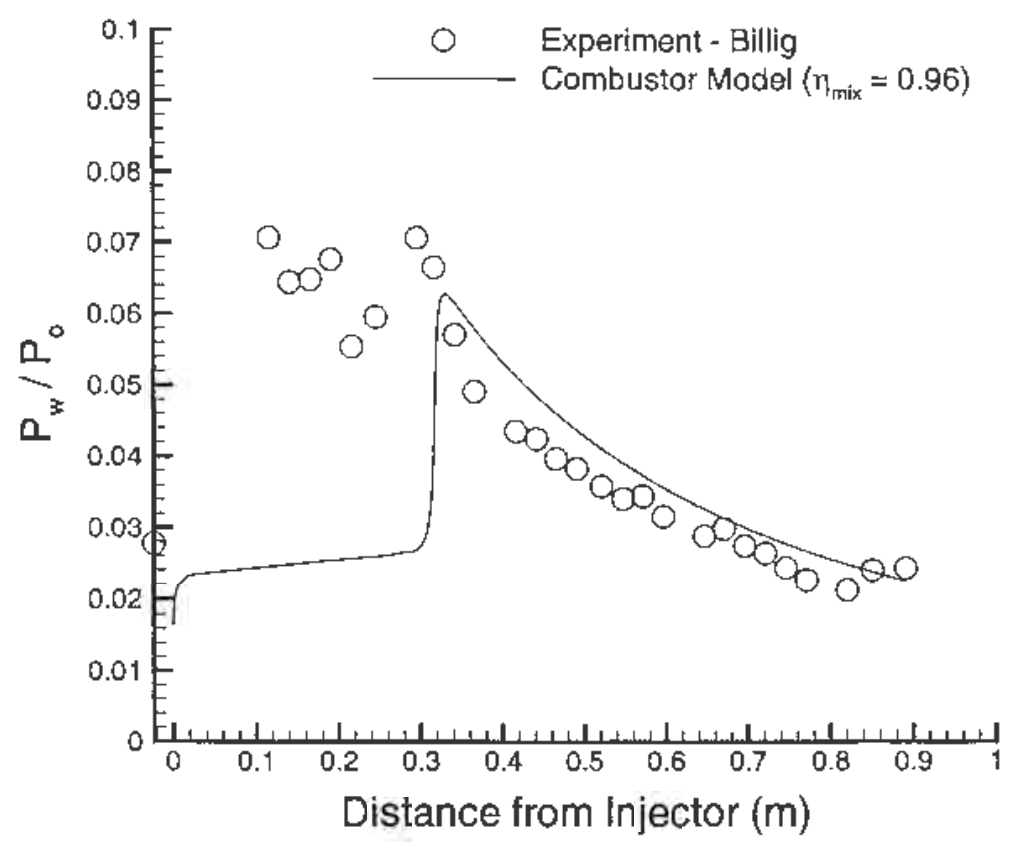

Figure 3.12: Comparison of non-dimensional wall pressure between experimental results from Ref. 64 and current combustor model.

$872 \mathrm{~K}, \mathrm{~T}_{w}=500 \mathrm{~K}, \gamma=1.346$, and $\mathrm{M}=3.19$. The assumed properties were selected to fall within the range of typical operating values reported in Ref. 64 and to closcly match the reported incoming mass flow rate (an overprediction of about $6 \%$ ). From the selected values, the resulting error in total temperature is an overprediction of $10 \%$ and the resulting error in total pressure is an underprediction of $14 \%$. Considering the wide range of values reported in the literature, these errors are deemed acceptable for the current study.

Based on the above assumptions, the solution for the combustor flowfield is compared to the experimental results in Fig. 3.12. Good agrement is seen on the expansion region of the combustor, with an average error of under $15 \%$ compared to the experinental data. The pressure maximum is predicted at the $x=0.33$ $\mathrm{m}$ location (5 $\mathrm{cm}$ past the beginning of the expansion region). This location 
compares favorably with the experimental results for peak pressure location. Compared to an equilibrium solution, where the maximum in pressure would be just prior to the expansion region, the finite-rate solution allows for accurate prediction of fuel ignition while maintaining the peak pressure prediction found using equilibrium assumptions ${ }^{64}$ ( $10 \%$ error compared to experimental results).

Prior to the ignition point, the combustor model fails to predict the pressure along the wall of the combustor. It is believed that boundary layer burning may have occurred in this experiment ${ }^{65}$, which would increase the local pressure near the wall and act as a heat addition term. This effect, as well as any boundary layer separation due to injection, are not considered in the current combustor model. As a way to increase the heat addition, the mixing length $L_{\text {mix }}$ was assumed to be $2 \mathrm{~cm}$ in an attempt to model the above effects. A mixing efficiency of $96 \%$ was assumed for the above calculations to yield a combustion efficiency of $94 \%$ (the same as reported in Ref. 64 ). Varying the mixing efficiency by \pm $4 \%$ resulted in a change in the peak pressure of $\pm 3 \%$ and a change in pressure along the thrusting surface of $\pm 2 \%$.

The second experiment compared to the combustor model is that of the strutbased hydrogen fueled scramjet experiment by Anderson and Gooderum ${ }^{66}$, shown in Fig. 3.13. The apparatus consists of a strut injector which injects hydrogen normal to the flowfield. The strut is located in a constant area rectangular duct of height $3.81 \mathrm{~cm}$ and width $17 \mathrm{~cm}$. The injectors are located $24 \mathrm{~cm}$ from the expansion section of the combustor, which has a length of $47.9 \mathrm{~cm}$ and an exit height of $7.62 \mathrm{~cm}$. Ignoring the effects of the strut on the flowfield, the incoming flowfield has the following properties: $\mathrm{U}=1774.5 \mathrm{~m} / \mathrm{s}, \mathrm{p}=75777 \mathrm{~N} / \mathrm{m}^{2}, \mathrm{p}_{o}=$ $2.01 \mathrm{MN} / \mathrm{m}^{2}, \mathrm{~T}=1031 \mathrm{~K}, \mathrm{M}=2.7, \gamma=1.3$, an assumed $\mathrm{T}_{w}=500 \mathrm{~K}, L_{\text {mix }}$ $=60 \mathrm{~cm}, \eta_{\text {mix }}=95 \%, f_{s t}=0.0349, \phi=0.619$, and $22.3 \% \mathrm{O}_{2}, 46.5 \% \mathrm{~N}_{2}$ and $31.2 \% \mathrm{H}_{2} \mathrm{O}$ by volume. Reported experimental uncertainties are incoming Mach number of $\pm 0.05, \pm 5 \%$ variation in local concentration of burner fuel, and a 


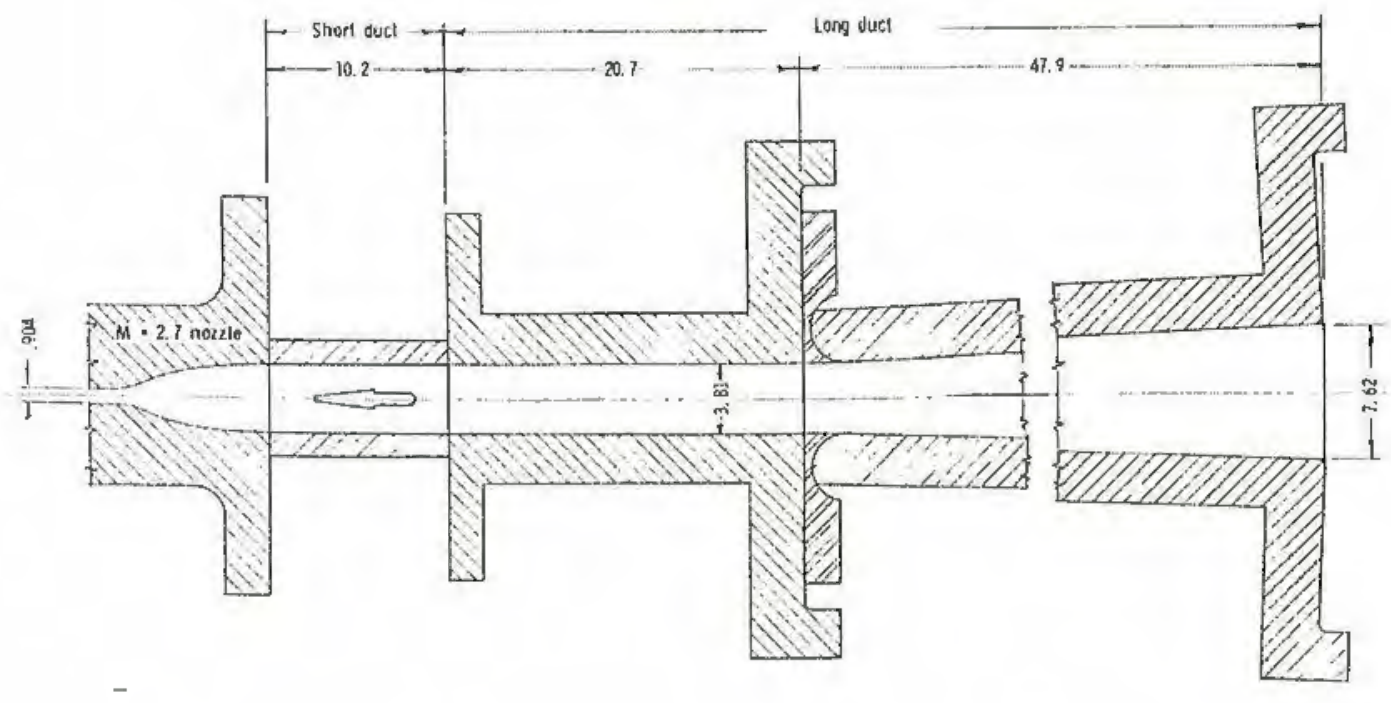

Figure 3.13: Experimental configuration for Ref. 66.

local stagnation temperature variation of $\pm 75 \mathrm{~K}$.

The results from the combustor model are compared to the experimental results of Ref. 66 in Fig. 3.14. Good agreement is again seen on the expansion portion of the combustor wall with errors under $13 \%$. Compared to the equilibrium solution reported by Anderson (see Fig. 3.14), a higher peak pressure is predicted which comes closer to predicting the peak pressure measured in the experiment (error of approximately 7.5\%). Prior to fuel ignition, the combustor model does not accurately predict wall pressures. The pressure data points in this region were influenced by boundary layer separation from shock impingement from the strut and the influence of fuel injection and burning. These effects are not modeled in the quasi-one-dimensional combustor model. Heat transfer to the duct was calculated to be $0.587 \mathrm{MJ} / \mathrm{s}$ compared to the experimental result of $0.718 \mathrm{MJ} / \mathrm{s}$, resulting in an $18 \%$ error. These results are seen to be in good agreement, considering that the combustor model did not include the length of duct prior to the strut injector, as well as the additional heat transfer from the shock wave/boundary layer interaction. 


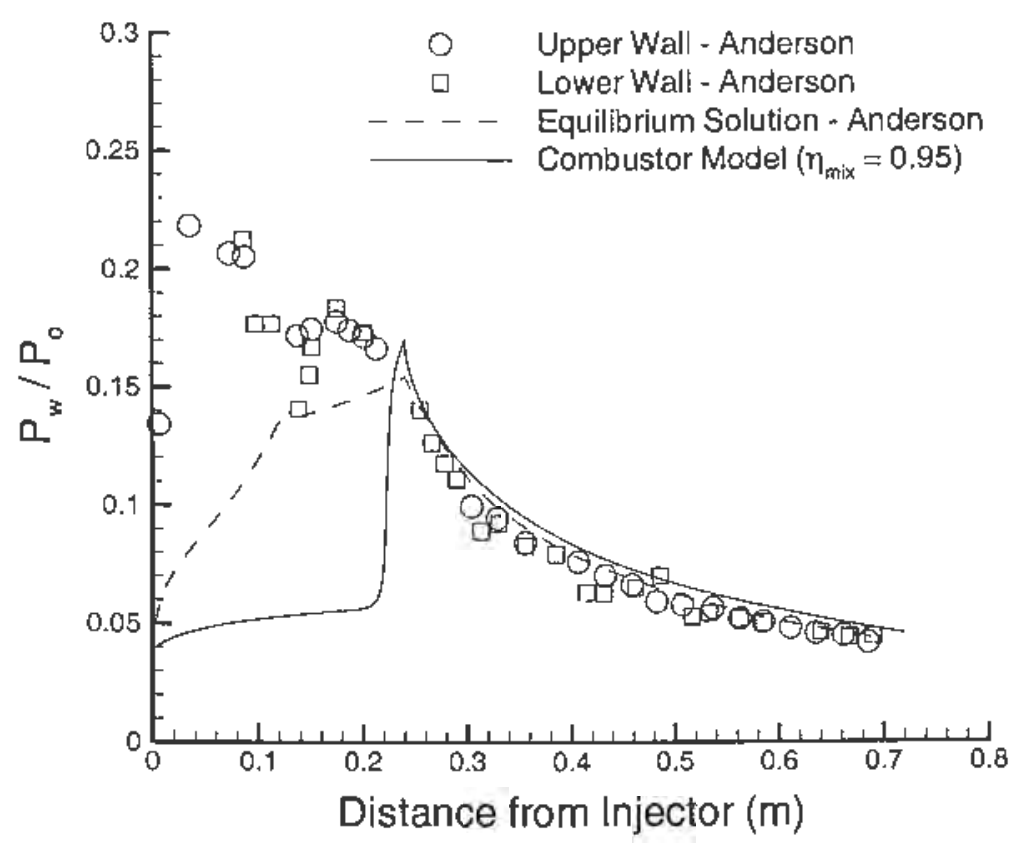

Figure 3.14: Comparison of non-dinnensional wall pressure between experimental results from Ref. 66 and current combustor model.

The mixing efficiency for this cass was selected to be $95 \%$. Based on the variation of local burner fuel concentration discussed above, a mixing efficiency of $95 \%$ was deemed as an optimistic assumption of fuel mixing efficiency. If the mixing efficiency is raised above this amount, the flow was found to thermally choke. For a mixing efficiency of $90 \%$, the peak pressure was reduced by $10 \%$ and the pressure along the thrusting surface was reducod by approximately $3 \%$.

Even with the vast limitations of quasi-onc-dimensional flow assumptions, the current modei does an excellent job of rapidly prodicting the pressure profile on the expansion surface (vital for thrust prediction). It also predicts fuol ignition location, even without the additional heat term clue to boundary layer burning. Peak pressures calculated also compare quite well witl experiment and previous equilibrium solutions. 


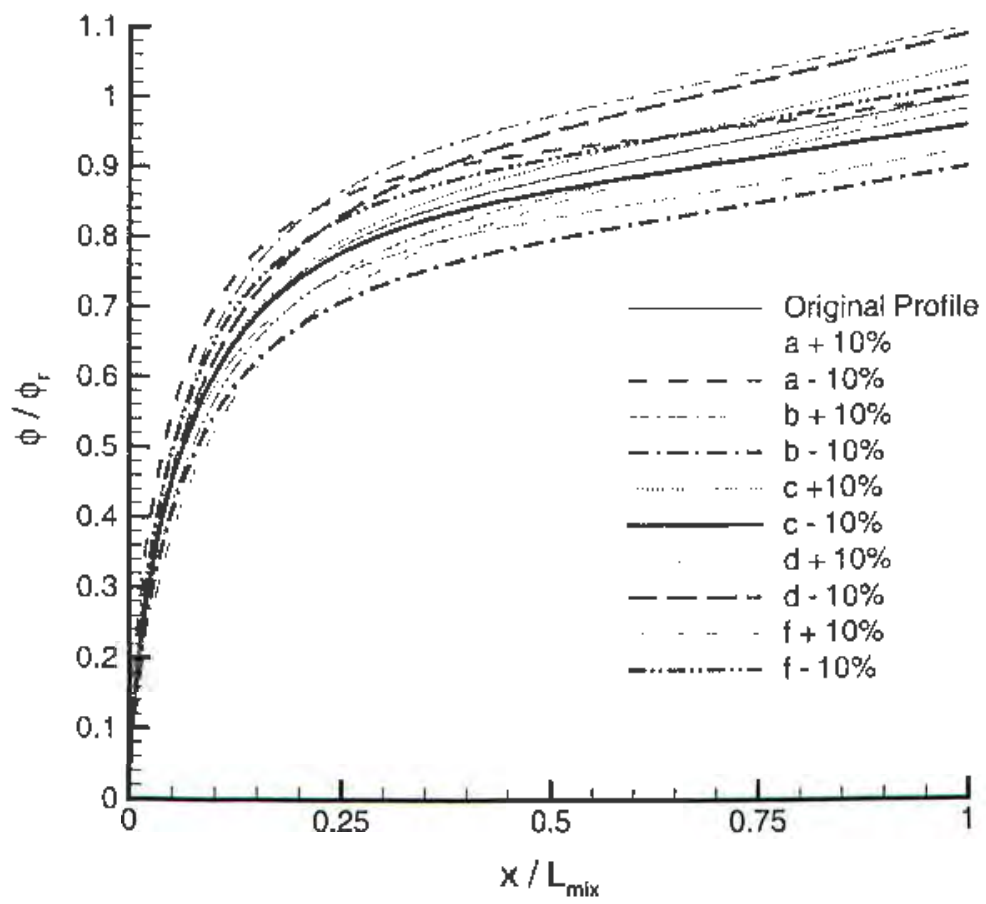

Figure 3.15: Sensitivity of anixing profile to perturbation of dependent coefficients.

\subsubsection{Sensitivity of Engine Model to Fuel Injection Profile}

The mixing profile defined by Eq. 3.32 is based on a curve fit of experimental data. It is prone to errors from the curve fit process, errors in the experiment, and errors in extrapolation from applying it to different combustor configurations. The coefficients of the profile $(a, b, c, d$, and $f)$ are perturbed so that different mixing profiles are developed. Each coefficient is porturbed $\pm 10 \%$, while leaving the others constant, as shown in Fig. 3.15. Except for coeflicient $a$, the result of perturlying the other coefficients results in injection of more or less fuel than specified (i.e., the mixing efficiency is changed). Thus, the perturbation of coefficient $a$ is the only true mixing profile variation that does not affect 


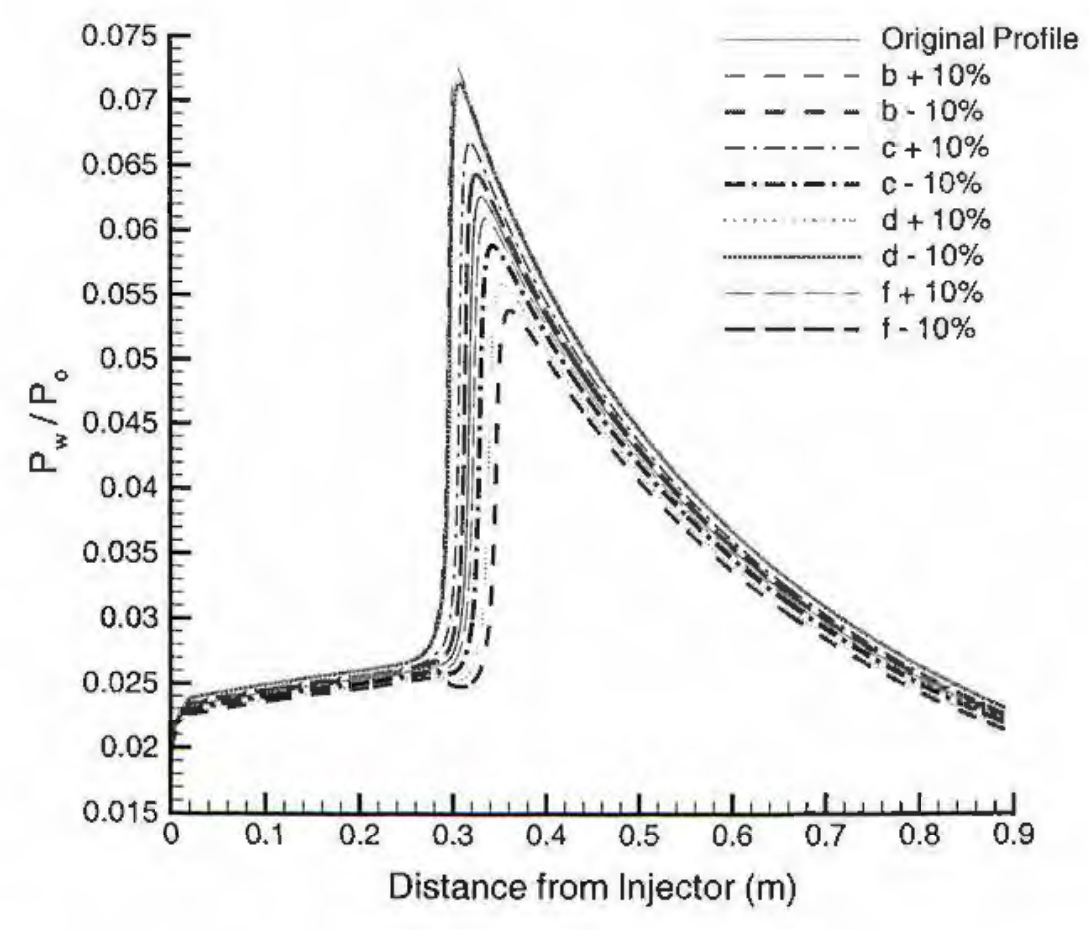

Figure 3.16: Sensitivity of engine model to mixing profile using Billig's engine profile.

the amount of fuel added to the combustor.

The effects of perturbing the coefficients of the mixing profile on the combustor flowfield are plotted in Fig. 3.16 for Billig's experimental configuration. It is noted that the perturbation of coefficient $a$ did not affect the flowfield. Hence, when the same amount of fuel is injected, a change in profile did not affect the overall solution. The effects of perturbing the other coefficients on the combustor flowfield are based on changes in the amount of fuel added (i.e., changing mixing efficiency). The quantitative changes in ignition distance, peak pressure, and exit pressure compared to the original profile are tabulated in Table 3.1. An interesting result found is that by perturbing each coefficient by $\pm 10 \%$ resulted in a maximum change in exit pressure of $\pm 4 \%$. 


\begin{tabular}{|c|c|c|c|}
\hline \multirow{2}{*}{} & \multicolumn{3}{|c|}{$\%$ Difference } \\
\cline { 2 - 4 } & Ignition Distance & Peak Pressure & Exit Pressure \\
\hline$b+10 \%$ & -6.6 & 15.9 & 4 \\
$b-10 \%$ & 8.5 & -14 & -4.2 \\
\hline$c+10 \%$ & -3.8 & 6.6 & 1.8 \\
$c-10 \%$ & 3.3 & -6 & -1.7 \\
\hline$d+10 \%$ & 6.3 & -10.7 & -3.1 \\
$d-10 \%$ & -3.3 & 14 & 3.5 \\
\hline$f+10 \%$ & 1.4 & -2.6 & -0.7 \\
$f-10 \%$ & -1.4 & 2.8 & 0.8 \\
\hline
\end{tabular}

Table 3.1: Sensitivity of combustor properties to mixing profile.

The fuel mixing model used in the combustor studies assumes normal fuel injection. Normal fuel injection in a supersonic flow causes normal shock waves, boundary layer separation, and localized heating. These detrimental effects increase with increasing combustor Mach number, reaching a point where normal fuel injection is no longer feasible. Considering the effects of normal injection, a mixing model based on axial mixing ${ }^{38}$ may be used

$$
\dot{m}_{r}=\dot{m}_{f} \frac{1-\exp \left(-A_{c} \bar{x}\right)}{1-\exp \left(-A_{c}\right)}
$$

where $A_{c}$ is injector geometry dependent and varies between 1 and 5 . Taking the derivative of Eq. 3.38 results in

$$
\frac{d \dot{m}}{d x}=\frac{\dot{m}_{r}}{L_{\text {mix }}} \frac{A_{c}}{\exp \left(A_{c} \bar{x}\right)-1}
$$

Assuming an identical mixing length (to show the effects of mixing profile on the solution), the axial injector mixing model described by Eqs. 3.38 and 3.39 


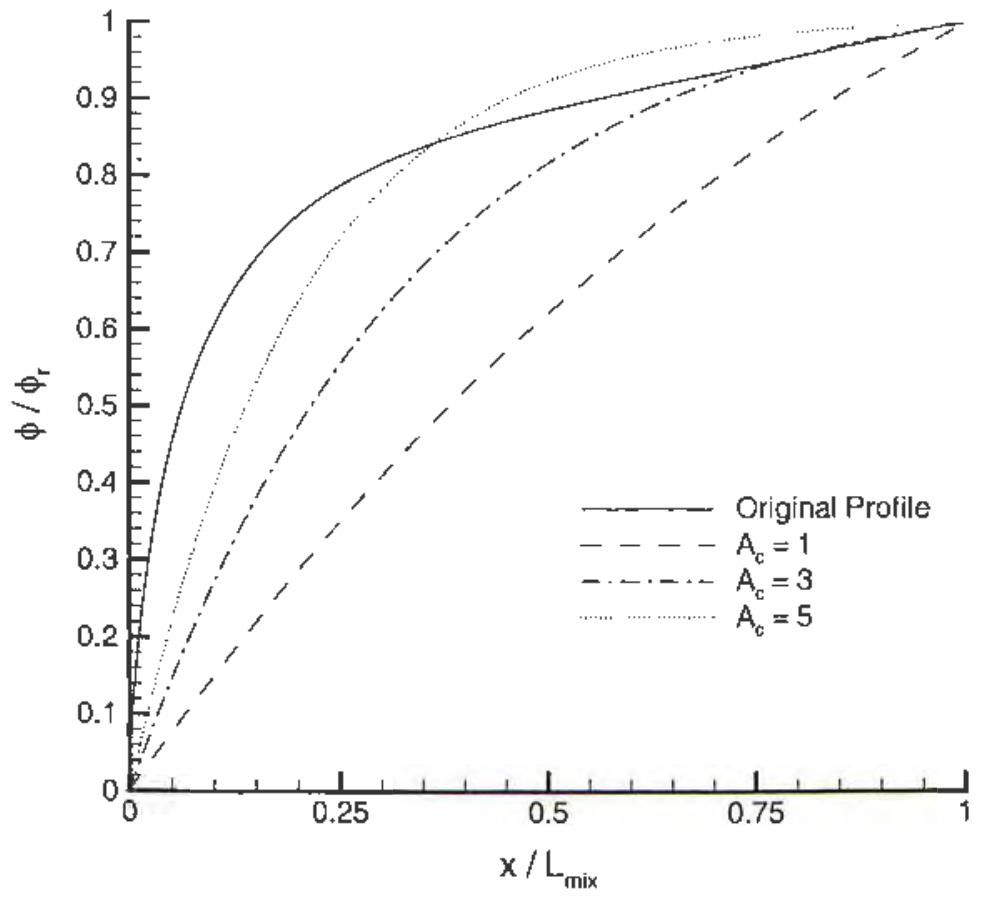

Figure 3.17: Comparison of normal to axial fuel injection prohles.

are applied to Billig's experimental contiguration. Fig. 3.17 shows the original nomal injection profile as well as the profile described by Eq. 3.38 for three values of $A_{c}: 1,3$, and 5. For each profile, the resulting pressure distribution was virtually unchanged (except for the profile in the mixing region). Since the mixing length remained constant (and is small for this application), tho effect of mixing profile was minimal.

\subsubsection{Example Rocket-Based Combined Cycle Engine Solution in Scramjet Mode}

An example scramjet-mode flowheld solution is now provifed. The top view of a portion of the engine in scramjet mode is shown in Fig. 3.18. The area- 


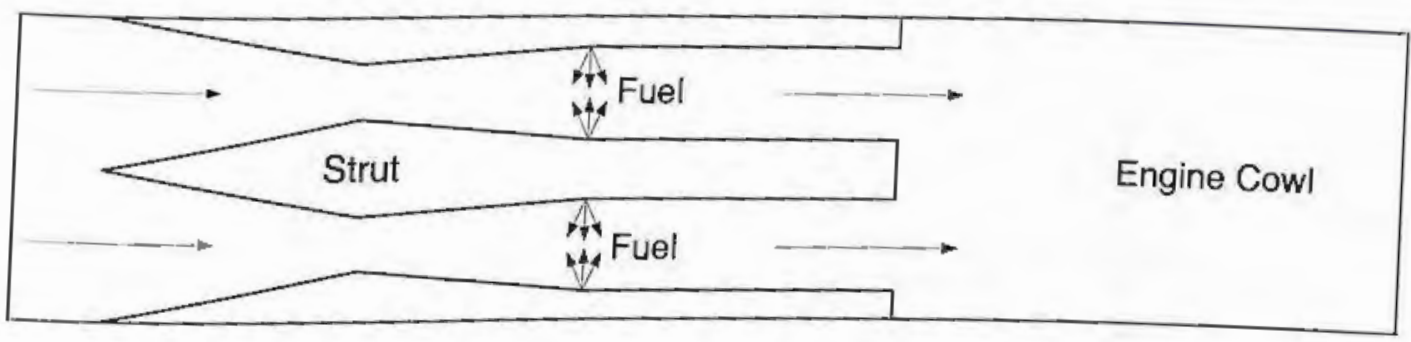

Figure 3.18: RBCC engine scramjet mode geometry, top view (not to scale).

averaged results from the compression region (see Section 3.1) are placed into the one-dimensional combustor model presented above to calculate the combustor flowfield. The chemical composition entering the combustor is assumed to be the chemical composition of air discussed in Section 3.2.7. Gaseous hydrogen is injected normally at the end of the expansion portion of the strut (see Fig. 3.18). The fuel is assumed to be fully mixed by the end of the constant area region between each set of struts, and follows the same mixing profile as discussed in the above section. Following the constant area region, a short but finite expansion is assumed to model the base of the struts. Base pressure on the strut is assumed to be an average of the pressure before and after the expansion. Following the end of the combustor, the expansion flowfield is calculated using the method of characteristics as described in Section 2.3.

Assuming a freestream Mach number of 12 at an altitude of $36 \mathrm{~km}$, the areaaveraged incoming properties into the combustor are: $\mathrm{p}=0.3 \mathrm{~atm} . \mathrm{T}=980 \mathrm{~K}$, $\mathrm{M}=5.73, \gamma=1.34, \dot{m}=722 \mathrm{~kg} / \mathrm{s}, \phi=1, \eta_{\text {mix }}=95 \%$ (assumed), $T_{w}=1200 \mathrm{~K}$ (assumed), constant area section length of $2.6 \mathrm{~m}$, combustor expansion length of $3.17 \mathrm{~m}$, and a combustor expansion angle of $8.9^{\circ}$. The above conditions and geometry are provided as an example and do not imply any optimality.

The resulting distributions in Mach number and pressure are shown in Fig. 3.19. The Mach number is observed to decrease sharply due to mass injection at the beginning of the combustor. At a distance of approximately $0.25 \mathrm{~m}$, fuel ignition 


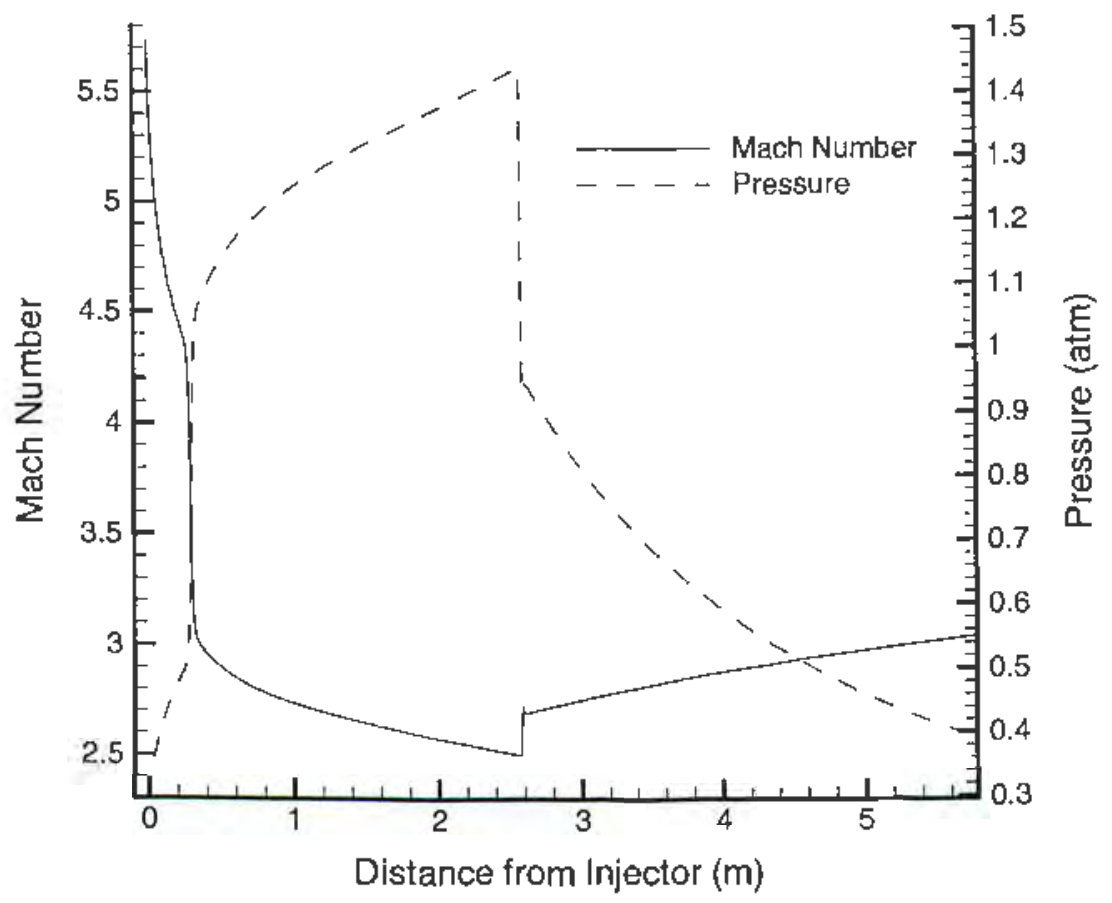

Figure 3.19: Mach munber and pressure distribution in RBCC engine in scramjet mode.

is observed with another sharp decrease in Mach number. The Mach number is seen to further decrease beyond fuel ignition due to combustion and friction effects. At the end of the strut, a sharp rise in Mach number is seen due to the expansion from the base of tho strut. The Mach number then further rises after the strut due to the constant angle expansion region of the combustor. The pressure trend is seen to mirror the effects secn in the Mach number trend (as Mach number gocs down, pressure increases).

The resulting distribution in temperature and mass fractions of $\mathrm{H}_{2} \mathrm{O}, \mathrm{OH}$, and NO are shown in Fig. 3.20. A rise in temperature is seen for both mass injection and for fuel ignition in the same manner as Mach number. It is noted that after the strut injector base, the temperature is seen to rise instead of fall as 


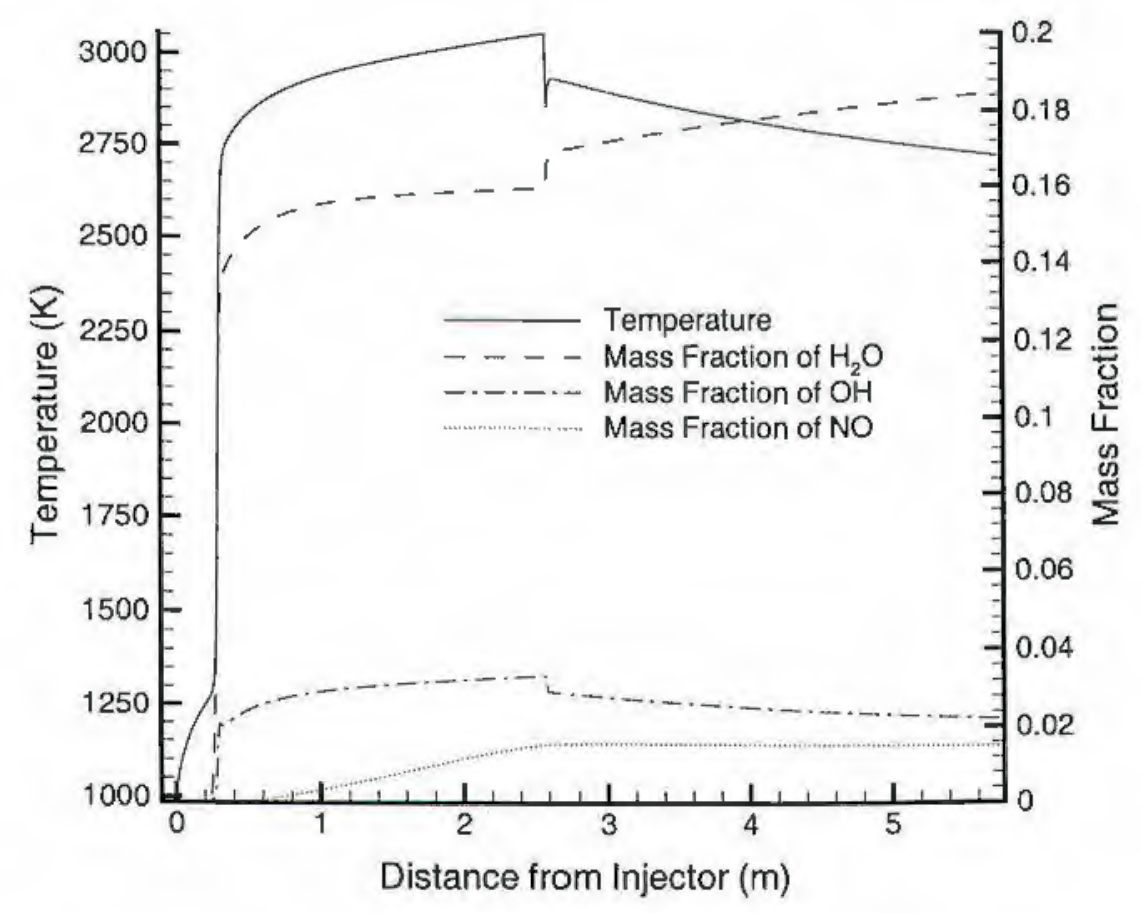

Figure 3.20: Temperature and mass fraction of $\mathrm{H}_{2} \mathrm{O}, \mathrm{OH}$, and $\mathrm{NO}$ distribution in RBCC engine in scramjet mode.

would be expected in a supersonic expansion. Observing the water mass fraction, it is seen that at the strut base expansion, water is being created. Due to the high combustion temperatures in the constant area region of the combustor, dissociation of water is occurring (observe the $\mathrm{OH}$ mass fraction). When the flow cools down through the initial expansion, water can begin to form. As the expansion becomes less severe (i.e., after the base), the heat release from water formation is still high enough to heat the flow. As the flow continues in the expansion region, fewer reactions occur and the mass fraction begins to level out. Consequently, the temperature falls because of the expansion, and the expected result of temperature decrease is observed.

The combustion efficiency in this study is defined as the mole fraction of 


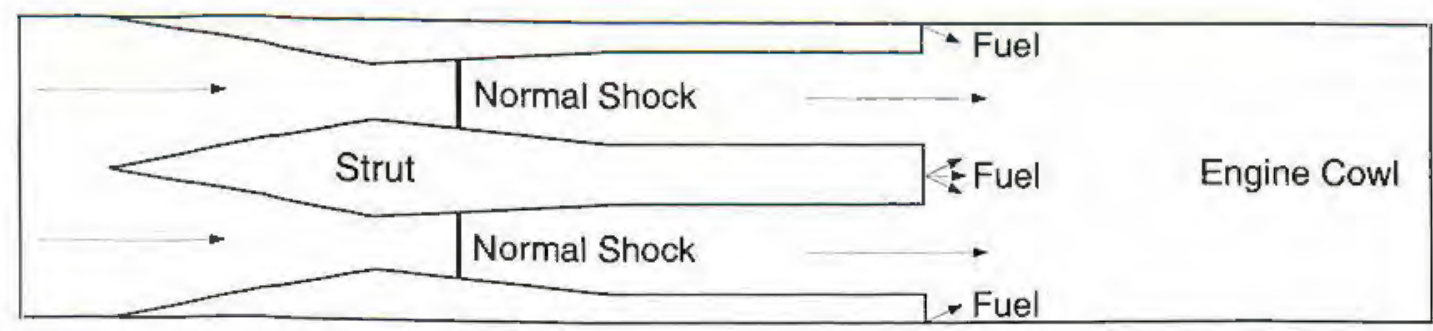

Figure 3.21: RBCC engine ramjet mode geometry, top view (not to scale).

water at the exit of the combustor divided by the stoichiometric mole fraction of water if all of the hydrogen has been converted to water. In this manner, the combustion efficiency for this particular case was found to be about $69 \%$. This low value of combustion efficiency is partially due to the high combustion temperatures $(\sim 3000 \mathrm{~K})$ in the burner keeping some of the water dissociated, and formation of NO (see Fig. 3.20) which reduces the amount of available oxygen for reaction. The low value of efficiency is also a result of the high expansion angle used in the combustor, freezing the reactions before they can come closer to completion.

\subsection{Ramjet Mode}

An example ramjet mode flowfield solution is now provided. The top view of a portion of the engine in ramjet mode is shown in Fig. 3.21. The incoming conditions are found from the flow immediately following shock cancellation (see Fig. 3.1). The flow is then compressed between each set of struts. Following the throat of the struts, a normal shock wave exists, reducing the engine Mach number to subsonic speed. The flow then travels through the rest of the strut and exhausts into the constant angle expansion section. As the flow exhausts the struts, it is assumed to have a finite expansion due to the base of the strut (base pressure on the strut is calculated in the same manner as the scramjet solution). 
In this finite expansion region, fuel is injected from the base of the struts and is assumed to be fully mixed. The flow then goes through the constant angle expansion where combustion occurs and a thermal throat is established. It is in this region that the flow transitions from subsonic to supersonic flow. At the end of the engine, the flow is fully supersonic and is expanded through the method of characteristics nozzle to the end of the vehicle.

The compression flowfield between each strut is assumed to be quasi-onedimensional, viscous, and is solved using the combustor model developed above. Observing the trends found in Fig. 3.8, as engine inlet Mach number decreases, the results using quasi-one-dimensional flow approach the CFD and analytical model values. The inlet Mach number for the ramjet will be low due to the freestream Mach number in which the ramjet operates. The flow is compressed, passes through the throat, and continues until it reaches the normal shock wave. The flow is compressed through the normal shock wave, and then is assumed to be viscous and quasi-one-dimensional to the end of the strut.

The normal shock wave exists within the strut compression flowfield to raise the pressure to the back pressure in the combustor. The back pressure is determined by the pressure of the flowfield at the thermal throat. Since it is unknown a priori what conditions will necessitate a thermal throat, normal shock location as well as fuel equivalence ratio are iterated to establish a thermal throat within the constant angle expansion section. Thus, when the iteration is complete, the normal shock wave is located at the right location between the struts to produce the back pressure found at the thermal throat.

In reality, a normal shock wave within the strut compression flowfield may not exist as found in Fig. 3.21. The boundary layers between the struts will separate due to the adverse pressure gradient from the shock wave. The boundary layer will thicken and cause the flow to accelerate to supersonic speeds, which will cause another normal shock wave to form. This process repeats itself until 
the pressure behind the last normal shock wave is equivalent to the pressure necessary to obtain the back pressure at the thermal throat. The series of normal shocks within the compression flowfield is known as a normal shock train, and is a complicated phenomenon to predict. However, an empirical correlation by Waltrup et al. ${ }^{67}$ may be used to approximate the isolator length necessary to contain a normal shock train with compression ratio of $p_{e} / p_{i}$

$$
\frac{L_{\text {isolator }}}{H_{\text {isolator }}}=\frac{\sqrt{\frac{\bar{\theta}}{H_{\text {isolator }}}}}{\sqrt[4]{\operatorname{Re} e_{\bar{\theta}}}} \frac{50\left(\frac{p_{e}}{p_{i}}-1\right)+170\left(\frac{p_{e}}{p_{i}}-1\right)^{2}}{M_{i}^{2}-1}
$$

where $L_{\text {isolator }}$ and $H_{\text {isolator }}$ are the length and height of the isolator, respectively, $\bar{\theta}$ is the boundary layer momentum thickness, $R e_{\bar{\theta}}$ is the Reynolds number based on the boundary layer momentum thickness, $p_{e} / p_{i}$ is the compression ratio across the entire isolator, and $M_{i}$ is the ramjet inlet Mach number. Suggested values $^{38}$ for an initial estimation of isolator geometry are a momentum thickness of $0.02 H_{\text {isolator }}$ and a Reynolds number of 10,000.

Equation 3.40 is meant for normal shock trains only. As inlet Mach number increases, the shock train will transition to an oblique shock wave train. For this work, the shock train is assumed to be modeled by one normal shock wave, and does not transition to an oblique shock train at higher inlet Mach number. Using the suggested values listed above, an isolator length-to-height ratio of over 10 can easily be expected. However, in the current work, the isolator length is not restricted by Eq. 3.40. In the following discussion on the ramjet solution, it will be noted when the isolator length used violates the approximation given by Eq. 3.40.

Assuming a freestream Mach of 5 at an altitude of $24.7 \mathrm{~km}$, the flowfield properties entering into the isolator section are: $\mathrm{p}=0.16 \mathrm{~atm}, \mathrm{~T}=378 \mathrm{~K}, \mathrm{M}$ $=3.49, \gamma=1.4, \dot{m}=548 \mathrm{~kg} / \mathrm{s}, \phi=0.944, \eta_{\text {mix }}=100 \%$, and $T_{w}=T_{\text {local }} . A$ normal shock wave is located $0.01 \mathrm{~m}$ behind the throat of the struts. With these properties, the isolator length requirement approximated by Eq. 3.40 is violated. 


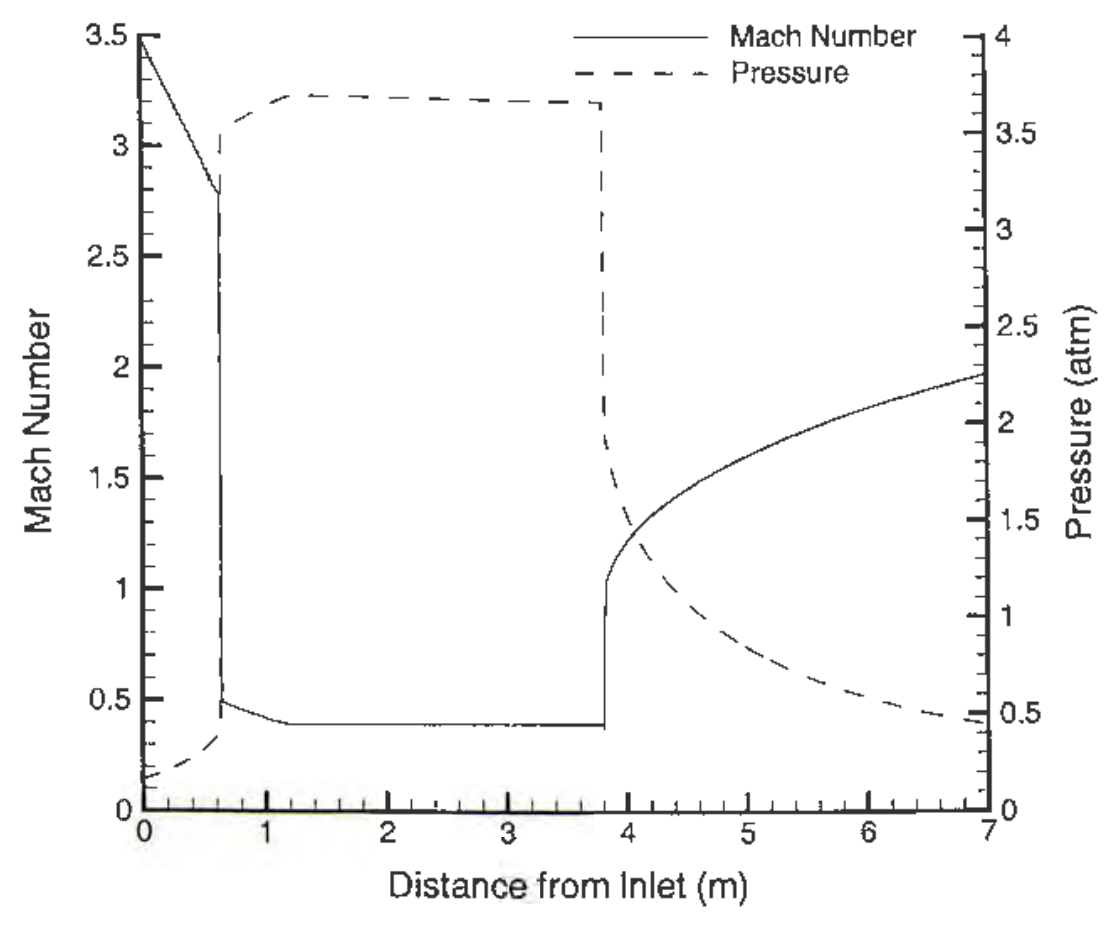

Figure 3.22: Mach number and pressure distribution in RBCC engine in ramjet mode.

The above conditions and geometry are provided as an exanple and do not imply any optimality.

The resulting distributions in Mach number and pressure in the ranjet are shown in Fig. 3.22. The Mach number is observed to decrease upon entering the isolator and continues to decrease until the throat. The decrease in Mach number is from the compression of the struts as well as viscous effects. Following the throat, a normal shock wave lowers the Mach number to subsonic speeds. The flow then continues to decelerate from the expansion portion of the struts. The Mach number is observed to have virtually no change through the constant area section. As the flow exhausts the isolator section and expands over the base of the strut, the fucl added to the flow ignites, causing the Mach number 


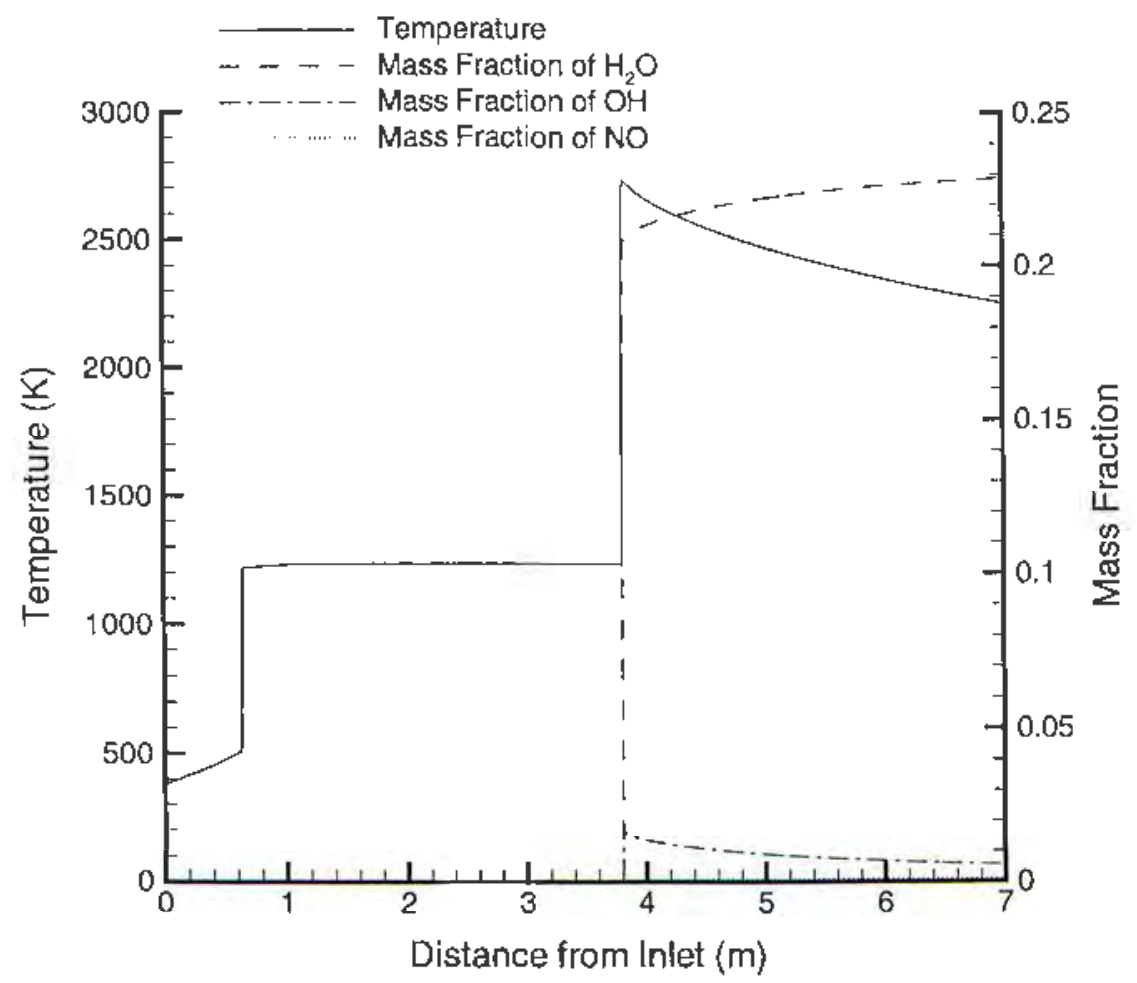

Figure 3.23: Temperature and mass fraction of $\mathrm{H}_{2} \mathrm{O}, \mathrm{OH}$, and $\mathrm{NO}$ distribution in $R B C C$ engine in ranjot mode.

to rise. As the flow continues in the constant angle expansion section, a thermal throat is established, followed by supersonic accelerntion through the remainder of the engine. The pressure in the ramjet follows an opposite pattern as the Mach number, increasing in the isolator and through the normal shock, then decreasing from combustion and expansion.

The resulting distributions in temperature and mass fractions of $\mathrm{H}_{2} \mathrm{O}$, OH, and NO are shown in Fig. 3.23. The temperature profile follows the same trends as the pressure profile within the isolator. The tomperature increases from compression and viscous effects, jumps across the normal shock wave, then slightly increases through the rest of the isolator from viscous effects. As the flow exhausts the isolatoss, fuel is injected and the mixture begins to burn. 'The flow 
goes through a thermal throat, then the temperature begins to decrease due to supersonic expansion through the constant angle expansion section.

The combustion efficiency for this case was found to be approximately $93 \%$ using the same methodology as the scramjet example. Compared with the scramjet, the ramjet was found to have a much higher efficiency. The increase in efficiency is from an increase in assumed mixing efficiency as well as a decrease in combustion temperature. Observing the mass fraction trends, the mass fractions of $\mathrm{OH}$ and $\mathrm{NO}$ are substantially reduced compared to the scramjet solution. These products have lower mass fractions because of the decreased combustion temperature, which allows less $\mathrm{H}_{2} \mathrm{O}$ to dissociate.

A problem found with this ramjet model was that combustion could not be sustained for freestream Mach numbers below 4.5. This was due to a combination of low temperatures exiting the isolator and higher Mach number behind the normal shock wave. Two crucial aspects to a ramjet design that were not incorporated into the current model are a flameholder and a variable inlet. Both of these devices are implemented on ramjets to increase their efficiency. The flameholder aids in fuel mixing and fuel ignition, while the current model relies on flow conditions from the isolator to ignite the fuel. A variable inlet allows for better compression efficiency as well as better mass flow capture, the current model is static, except for the engine cowl which translates to maintain shock cancellation. While the engine model has been shown to work for ramjet operation, improvements to the model will be needed to deliver more efficient performance. 


\section{Chapter 4}

\section{Transonic Off-Design Aerodynamic Performance}

An important aspect to predicting the performance of the vehicle is its off-design aerodynamics for both angle of attack and Mach number. In this chapter, the methods used to predict the aerodynamics on the vehicle geometry for transonic off-design conditions are discussed. These methods are applied to a full vehicle geometry and validated using $3-\mathrm{D}$, inviscid computational solutions over the fuselage.

\subsection{Two-Dimensional Flow Solution}

As a review of previous work performed, the transonic, small disturbance equation in two-dimensions ${ }^{68}$ may be written as

$$
\left(1-M_{\infty}^{2}-M_{\infty}^{2} \frac{\gamma+1}{U_{\infty}} \phi_{x}\right) \phi_{x x}+\phi_{z z}=0
$$

This equation simplifies to the linearized perturbation velocity potential equation if the coefficient of $\phi_{x}$ is neglected. However, this coefficient must be retained in the transonic equation to remain of mixed type (hyperbolic, parabolic, or elliptic). The governing equation changes types according to the coefficient of 
$\phi_{x x x}$

$$
1-M_{\infty}^{2}-M_{\infty}^{2} \frac{\gamma+1}{U_{\infty}} \phi_{x} \begin{cases}<0 & \text { hyperbolic } \\ =0 & \text { parabolic } \\ >0 & \text { elliptic }\end{cases}
$$

Ilonce, Eq. 4.1 mimies the properties of transonic fow (How where transition from subsonic to supersonic occurs).

Two boundiry conditions are necessary for the solution of Ecl. 4.1. The first boundary condition is that the perturbation volocities go to zero at an infinite distance from the body. 'This implies that the perturbation velocity potential must be constant at infinity. The second boundary condition uses a small perturbation assumption to solve for the perturbation velocity potential at the surface of the shape

$$
\left[\phi_{z}\right]_{z=0}=U_{\infty} \frac{d Z}{d x}
$$

The pressure coefficient $C_{p}$ is the same as the linearized pressure coefficiont

$$
C_{p}=-2 \frac{\phi_{\pi}}{U_{\infty}}=-2 \frac{u}{U_{\infty}}=-2 \bar{u}
$$

where $u$ is the perturbation velocity in the $x$-direction and $\bar{u}$ is the non-rlimensional perturbation velocity in the $x$-direction.

The method of solution of the 2-D, transonic, small disturbanes equation selected for use in this study is the local linearization technique devoloped by Spreiter and Alksne ${ }^{i s}$. To familiarize the reader with the method, a summary of the technique prosented in Ref. 68 will be provided for the solution of flows that are purcly supersonic, purely subsonic, and near sonic. For a detailed discussion of the solutions to follow, the reader is relorred to Ref. 68 . 


\subsubsection{Supersonic Solution}

Equation 4.1 is condensed by using the following notation

$$
\lambda_{h} \equiv M_{\infty}^{2}-1+M_{\infty}^{2} \frac{\gamma+1}{U_{\infty}} \phi_{x}=M_{\infty}^{2}-1+k \bar{u}
$$

where $\lambda_{h}$ is positive, the perturbation velocity $u$ has been substituted for $\phi_{x}$, and

$$
k \equiv M_{\infty}^{2}(\gamma+1)
$$

The resulting equation is of hyperbolic type

$$
\phi_{z z}-\lambda_{h} \phi_{x x}=0
$$

The method of local linearization ${ }^{68}$ assumes that for the initial solution for $\phi$, the quantity $\lambda_{h}$ is a finite, positive quantity that varies slowly such that it may be considered a constant. With this assumption, the solution to Eq. 4.7 is the same as the linearized supersonic solution ${ }^{68}$

$$
\bar{u}=-\frac{1}{\sqrt{\lambda_{h}}} \frac{d Z}{d x}
$$

where $\mathrm{dZ} / \mathrm{dx}$ is the slope of the body and includes angle of attack. Taking the derivative of $\bar{u}$ with respect to $x$ while holding $\lambda_{h}$ constant, then substituting $\lambda_{h}$ into the resulting equation yields the following ordinary differential equation

$$
\frac{d \bar{u}}{d x}=-\frac{1}{\sqrt{M_{\infty}^{2}-1+k \bar{u}}} \frac{d^{2} Z}{d x^{2}}
$$

Equation 4.9 may then be integrated with respect to $\bar{u}$. The resulting equation has a constant of integration which is obtained by setting $\bar{u}=0$ where $d Z / d x=0$. The resulting value of $\bar{u}$ is then substituted into Eq. 4.4 to solve for the pressure coefficient

$$
C_{p}=\frac{2}{k}\left\{\left(M_{\infty}^{2}-1\right)-\left[\left(M_{\infty}^{2}-1\right)^{\frac{3}{2}}-\frac{3}{2} k \frac{d Z}{d x}\right]^{\frac{2}{3}}\right\}
$$


It is noted that the quantity in square brackets should be positive in order for the assumption of positive $\lambda_{h}$ not to be violated. However, for solutions where the term does become negative, the minus sign in front of the square bracket may be exchanged with a positive sign (and the absolute value of the square bracket term taken) to properly predict the trend, albeit at the cost of increased solution error. It is also possible to assume that the pressure coefficient remains constant in this region (unless the term is negative at the leading edge of the vehicle, in which case a subsonic solution is more applicable).

\subsubsection{Subsonic Solution}

Equation 4.1 is condensed by using the following notation

$$
\lambda_{e} \equiv 1-M_{\infty}^{2}-M_{\infty}^{2} \frac{\gamma+1}{U_{\infty}} \phi_{x}=1-M_{\infty}^{2}-k \bar{u}
$$

where $\lambda_{e}$ is positive. The resulting equation is of elliptic type

$$
\lambda_{e} \phi_{x x}+\phi_{z z}=0
$$

Again, the local linearization method ${ }^{68}$ assumes that for the initial solution for $\phi$, the quantity $\lambda_{e}$ is a finite, positive quantity that varies slowly such that it may be considered a constant. With this assumption, the solution to Eq. 4.12 is the same as that for a subsonic airfoil ${ }^{68}$

$$
\bar{u}=\frac{1}{\pi \sqrt{\lambda_{e}}} \int_{x_{\text {start }}}^{x_{\text {end }}} \frac{d Z}{d \xi} d \xi=\frac{\bar{u}_{i}}{\sqrt{\lambda_{e}}}
$$

where $\bar{u}_{i}$ is the nondimensionalized, incompressible solution for the perturbation velocity. As in the supersonic case, the derivative of $\bar{u}$ with respect to $x$ is taken while holding $\lambda_{e}$ constant, then $\lambda_{e}$ is substituted back into the equation. The result is an ordinary differential equation

$$
\frac{d \bar{u}}{d x}=\frac{1}{\sqrt{1-M_{\infty}^{2}-k \bar{u}}} \frac{d \bar{u}_{i}}{d x}
$$


Integrating Eq. 4.14 and calculating the constant of integration by setting $\bar{u}=0$ where $\bar{u}_{i}=0$ rosults in the solution for $\bar{u}$. Substitution into Eq. 4.4 results in the pressure coefficient

$$
C_{p}=-\frac{2}{k}\left\{\left(1-M_{\infty}^{2}\right)-\left[\left(1-M_{\infty}^{2}\right)^{\frac{3}{2}}+\frac{3}{4} k C_{p_{i}}\right]^{\frac{2}{3}}\right\}
$$

where $C_{p_{i}}$ is the incompressible pressure coefficient found from

$$
C_{p_{i}}=-\frac{2}{\pi} \int_{x_{\text {setrit }}}^{x_{\text {wend }}} \frac{\frac{d Z}{d \xi}}{x-\xi} d \xi
$$

In order to satisfy the requirement that $\lambda_{c}$ is positive, the quantity in square brackets in Eq. 4.15 must be positive. The same approach used in the supersonic solution to deal with any negative quantities may be applied to this case as well.

\subsubsection{Near-Sonic Solution}

Equation 4.1 is condensed by using the following notation

$$
\lambda_{p} \equiv M_{\infty}^{2} \frac{\gamma+1}{U_{\infty}} \phi_{x x}=k \frac{\partial \bar{u}}{\partial x}
$$

where $\lambda_{p}$ is positive for accelerating hows and negative for decelerating flows. The resulting partial differential equation is

$$
\phi_{z z}-\lambda_{p} \phi_{x}=\left(M_{\infty}^{2}-1\right) \phi_{x x}
$$

Equation 4.18 is of parabolic type if the Mach number is sonic, of hyperbolic type for Mach number grater than unity, and of elliptic type for Mach number less than unity. For positive $\lambda_{p}$ (nccelerating flow) the solution for $\bar{u}$ is found using Green's theorem, the local linearization method"s, Mach number near unity, and focusing the solution around the vicinity of the airfoilss

$$
\bar{u}=\frac{\left(1-M_{\infty}^{2}\right)}{k}-\frac{1}{\sqrt{\pi \lambda_{j}}} \frac{d}{d x} \int_{x_{\text {sLart }}}^{x_{\text {endrd }}} \frac{\frac{d Z}{d \xi}}{\sqrt{x-\xi}} d \xi
$$


Again, by substitution of $\lambda_{p}$ into Eq. 4.19, the result is an ordinary differential equation. The equation may then be integrated as before, and the constant of integration is found by assuming that Eq. 4.19 may be used to find the sonic location. The resulting expression for pressure coefficient may then be determined as

$$
C_{p}=-\frac{2\left(1-M_{\infty}^{2}\right)}{k}-2\left[\frac{3}{\pi k} \int_{x^{*}}^{x}\left(\frac{d}{d x_{1}} \int_{x_{\text {start }}}^{x_{1}} \frac{\frac{d Z}{d \xi}}{\sqrt{x_{1}-\xi}} d \xi\right)^{2} d x_{1}\right]^{\frac{1}{3}}
$$

where $x^{*}$ is the sonic location found by solving the following equation for $\mathrm{x}$

$$
\frac{d}{d x} \int_{x_{\text {start }}}^{x} \frac{\frac{d Z}{d \xi}}{\sqrt{x-\xi}} d \xi=0
$$

For the sonic cases, the near-nose region of the flowfield had a pressure distribution similar to a subsonic flow. Hence, the subsonic solution shown above is spline-fit into the sonic solution at an applicable location along the body. The spline is performed by changing the constant of integration so that the sonic and subsonic solution are equal at the selected point. The solution to the pressure coefficient in the splined region is then

$$
C_{p}=\frac{2}{k}\left\{\left[\left|1-M_{\infty}^{2}+\frac{k C_{p_{\times x}}}{2}\right|^{3 / 2}+\frac{3}{4} k\left(C_{p_{i}}-C_{p_{i, \times s}}\right)\right]^{2 / 3}-\left(1-M_{\infty}^{2}\right)\right\}
$$

where subscript " $\mathrm{xs}$ " denotes the value at the subsonic/sonic solution intersection point. Supersonic flow, near the trailing edge, may be similarly splined to the near sonic solution and is incorporated into the solutions that follow.

\subsection{Axisymmetric Flow Solution}

As a review of previous work performed, the axisymmetric, small disturbance, transonic, potential equation ${ }^{69}$ is stated as

$$
\left(1-M_{\infty}^{2}\right) \phi_{x x}+\frac{1}{r} \phi_{r}+\phi_{r r}=\frac{k}{U_{\infty}} \phi_{x} \phi_{x x x}
$$


where $r$ refers to the radial direction. The first boundary condition for this equation is that the velocity potential is constant at an infinite distance from the body (same as in two dimensions). The second boundary condition is the small disturbance assumption along the hody

$$
\left(r \phi_{r}\right)_{r=0}=\frac{U_{\infty}}{2 \pi} \frac{d S}{d x}
$$

where $S$ is the cross-sectional area of the body. The linearized pressure coefficient on the surface of the body is written as

$$
C_{p}=-2 \bar{u}-\left(\frac{d Z}{d x}\right)^{2}
$$

The method of solution of the axisymmetric, small disturbance, transonic, velocity potential equation selected in this study is the axisymmetric local linearization technique developed by Spreiter and Alksne ${ }^{69}$. Again, a summary of the solutions using this method for purely supersonic, purely subsonic, and near sonic is presented below to familiarize the reader with the axisymmetric method. A detailed discussion of the derivation of the following equations may be found in Ref. 69.

\subsubsection{Supersonic Solution}

Using the previous notation, Eq. 4.23 is condensed to the following form

$$
\lambda_{h} \phi_{x x}-\frac{1}{r} \phi_{r}-\phi_{r r}=0
$$

The solution for the perturbation velocity may be obtained by using the method of local linearization ${ }^{69}$

$$
\bar{u}=\frac{S^{\prime \prime}(x)}{4 \pi} \ln \lambda_{h}+f_{h}(x)
$$

where

$$
f_{h}(x)=\frac{S^{\prime \prime}(x)}{4 \pi} \ln \left(\frac{S(x)}{4 \pi x^{2}}\right)+\frac{1}{2 \pi} \int_{x_{\text {start }}}^{x} \frac{S^{\prime \prime}(x)-S^{\prime \prime}(\xi)}{x-\xi} d \xi
$$


Taking the derivative of $\bar{u}$ with respect to $x$ and stbstituting the value of $\lambda_{t}$ into the result, yiclds the following, nonlinear, ordinary differential eruation

$$
\frac{d \bar{u}}{d x}=\frac{S^{\prime \prime \prime}(x)}{4 \pi} \ln \left(\left|M_{\infty}^{2}-1+k \bar{u}\right|\right)+\frac{d f_{h}}{d x}
$$

where the absolute value of the matural logarithmic tem is used to guarantee a positive value. This equation may be munerically integrated to solve for the nondimensional perturbation velocity $\bar{u}$ using any standard ordinary differential equation solver. For the present study, all ordinary differential equations are solved using the $4^{\text {th }}$ order Runge-Kutta scheme ${ }^{15}$. To solve for the axisymmetric perturbation velocity for all three flow regimes, the geometries must be smooth to at least the third derivative (see Eq. 4.29). If this is not the case, large deviations in the second and third derivatives may cause large errors using this thicory.

In order to begin solution of Eq. 4.29, a starting value for $\bar{u}$ is needed at some $x$ location. In the supersonic case, this point is found by solving for $S^{\prime \prime}(x)=0$. The corresponding perturbation velocity is then found from Eq. 4.27

$$
\bar{u}=f_{l}
$$

Solution then proceds by starting at the above location and marching forward and backward to cover the complete length of the body. Another starting point that may be selected for a purcly supersonic solution is to calculate the pressure coefficient at the leading edge using the Taylor-Maccoll equation for supersonic flow over a cone. From the pressure coefficient, find the starting value for $\bar{u}$ and solve the ODE from the leading to the trailing edge. One final starting point (for sharp, cusped, trailing edges) is to set the pressure coefficient at the trailing edge to zero. This assurnption may be used since the How is assumed to be isentropic for the perturbation equations. All three of these starting points are used in the supersonic solution to follow. 


\subsubsection{Subsonic Solution}

Using the previous notation, Eq. 4.23 may be reduced to

$$
\lambda_{e} \phi_{x x}+\frac{1}{r} \phi_{r}+\phi_{r r}=0
$$

Using the method of local linearization ${ }^{69}$, the solution for the perturbation velocity may be found as

$$
\bar{u}=\frac{S^{\prime \prime}(x)}{4 \pi} \ln \lambda_{e}+\bar{u}_{i}
$$

where $\bar{u}_{i}$ is the incompressible perturbation velocity found from

$$
\bar{u}_{i}=\frac{S^{\prime \prime}(x)}{4 \pi} \ln \frac{S(x)}{4 \pi x\left(x_{\text {end }}-x\right)}+\frac{1}{4 \pi} \int_{x_{\text {start }}}^{x_{\text {end }}} \frac{S^{\prime \prime}(x)-S^{\prime \prime}(\xi)}{|x-\xi|} d \xi
$$

Taking the derivative of $\bar{u}$ with respect to $x$, and substituting in the value of $\lambda_{e}$ results in the nonlinear ordinary differential equation

$$
\frac{d \bar{u}}{d x}=\frac{S^{\prime \prime \prime}(x)}{4 \pi} \ln \left(\left|1-M_{\infty}^{2}-k \bar{u}\right|\right)+\frac{d \bar{u}_{i}}{d x}
$$

where the absolute value of the natural logarithmic term is used to guarantee a positive value. The initial point of solution is found by finding the value of $x$ that satisfies $S^{\prime \prime}(x)=0$, then finding the value of $\bar{u}=\bar{u}_{i}$ at $x$. Another starting point for the solution is to assume that the pressure coefficient at the trailing edge is zero (same reasoning as the supersonic solution) and to iterate the solution from trailing edge to leading edge. Both of these methods are employed in the solutions to follow.

\subsubsection{Near-Sonic Solution}

Using previous notation, Eq. 4.23 is simplified to the following form

$$
\frac{1}{r} \phi_{r}+\phi_{r r}-\lambda_{p} \phi_{x}=\left(M_{\infty}^{2}-1\right) \phi_{x x}
$$


Using the local lincarization technique ${ }^{6}$, assuming $\lambda_{p}>0$, applying Green's theorem to the surface of a slencier body, and near sonic Mach number, the solution for the perturbation velocity as is

$$
\bar{u}=\frac{1-M_{\infty}^{2}}{k}+\frac{S^{\prime \prime}(x)}{4 \pi} \ln \left(\frac{\lambda_{p} S(x) e^{C}}{4 \pi x}\right)+\frac{1}{4 \pi} \int_{J_{\text {sturt }}}^{\mathbf{x}} \frac{S^{\prime \prime}(x)-S^{\prime \prime}(\xi)}{x-\xi} d \xi
$$

where $C$ is Euler's constant $\left(C=0.577215 \ldots\right.$ ). Substituting $\lambda_{y}$, into Eq. 4.36 and solving for $d \bar{u} / d x$ yields the following nonlincar, ordinary differential equation

$$
\begin{array}{r}
\frac{d \bar{u}}{d x}=\frac{S^{\prime}(x) S^{\prime \prime}(x)}{4 \pi S(x)}+\exp \left\{\frac { 4 \pi } { S ^ { \prime \prime } ( x ) } \left[\bar{u}+\frac{M_{\infty}^{2}-1}{k}-\frac{S^{\prime \prime}(x)}{4 \pi} \ln \left(\frac{k S(x) e^{C}}{4 \pi x}\right)\right.\right. \\
\left.\left.-\frac{1}{4 \pi} \int_{x_{\text {start }}}^{x} \frac{S^{\prime \prime}(x)-S^{\prime \prime}(\xi)}{x-\xi} d \xi\right]\right\}
\end{array}
$$

Solution of $\mathrm{Eq} .4 .37$ requires a starting point, which is found by solving for the point at which $S^{\prime \prime}(x)=0$. However, when $S^{\prime \prime}(x)=0$ in Eq. 4.36, the solution is singular. Details of the determination of the initial solution point are discussed in Ref. 69. Reference 69 describes how the pressure distribution over the entire geometry may not be found using Eq. 4.37 alone. The sonic solution is splined to the supersonic and subsonic solutions (where applicable) to get the best solution. See Ref. 69 and the following sections for details.

\subsection{Application of Local Linearization Tech- nique to Prescribed Geometries}

A demonstration of the applicability of the local lincarization method for 2-D and axisymmetric flow is shown by comparing the tbeoretical results derived 


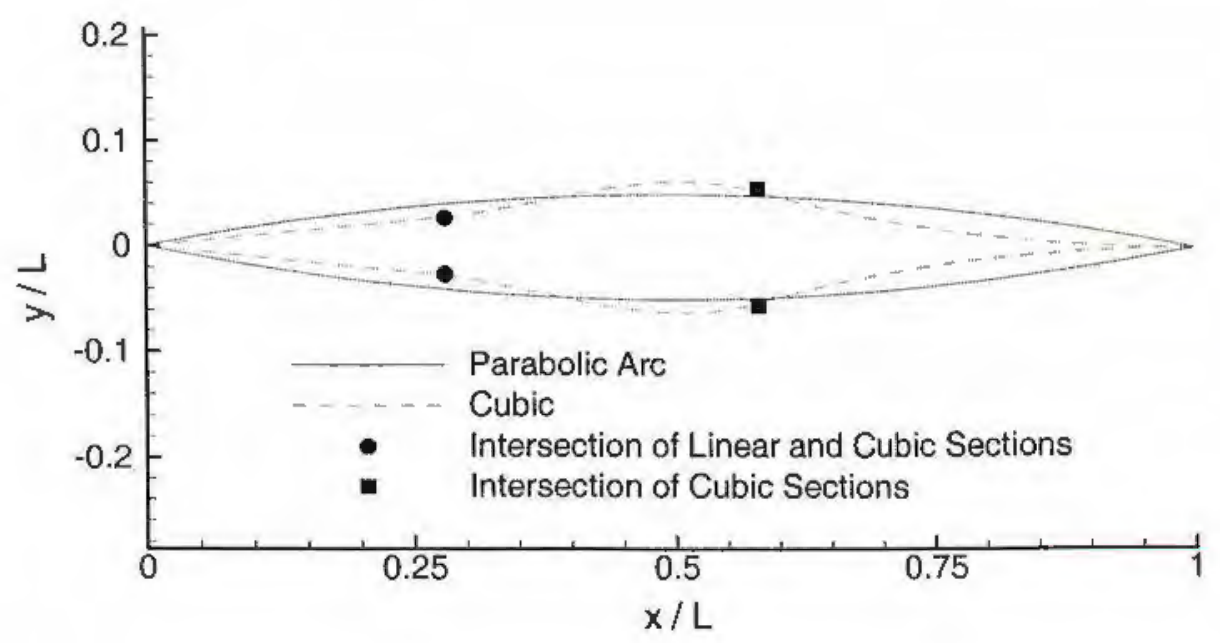

Figure 4.1: Profiles of geometries solved for validation.

above to computational calculations and experimental findings. Two geometries are investigated: a parabolic arc of the form

$$
Z=2 \tau c\left[\frac{x}{c}-\left(\frac{x}{c}\right)^{2}\right]
$$

and a geometry similar to the centerline of the compression region of the example in Fig. 2.1. The profiles solved are shown in Fig. 4.1, where the parabolic arc has a thickness ratio of $1 / 10$, the centerline geometry (labeled "cubic") has a thickness ratio of under 0.13 , and flow goes from left to right.

The cubic centerline geometry is composed of a linear front section followed by two cubic polynomials. The cubic sections are joined so that the slopes remain continuous at the intersection points. The intersection points of each of these sections are shown in Fig. 4.1. As noted in the solution of the axisymmetric equations, the assumption of a smooth profile (i.e., continuous derivatives in cross-sectional area) to the third derivative are necessary for a solution. However, when the original vehicle geometry ${ }^{70}$ was developed, interest was only paid to a continuous first derivative in cross-section. Hence, in order to use the theory developed, a seventh-order spline (the lowest order to guarantee continuous third 


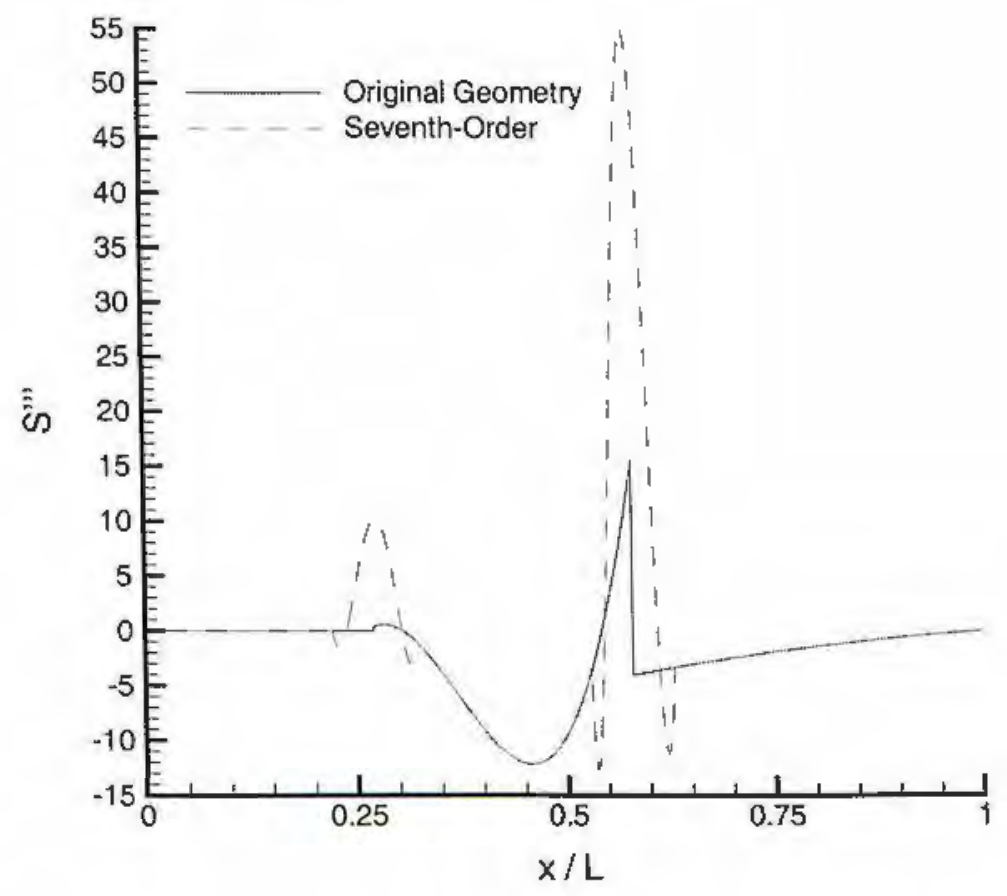

Figure 4.2: Third derivative of cross-sectional area for original geometry and seventh-order spline addition.

derivatives along the entire profile) was placed around the intersection points noted in Fig. 4.1. Each spline had a length of approximately $10 \%$ of the length of the vehicle, and produced no noticeable difference in appearance of the shape. A plot of the third derivative of cross-sectional area of the original shape and the shape with the seventh-order spline are shown in Fig. 4.2. With the spline, the third derivative in cross-sectional area is continuous, but has a wide variation in value over a small distance. This will result in errors for the prediction of the perturbation velocity for the axisymmetric cases. 

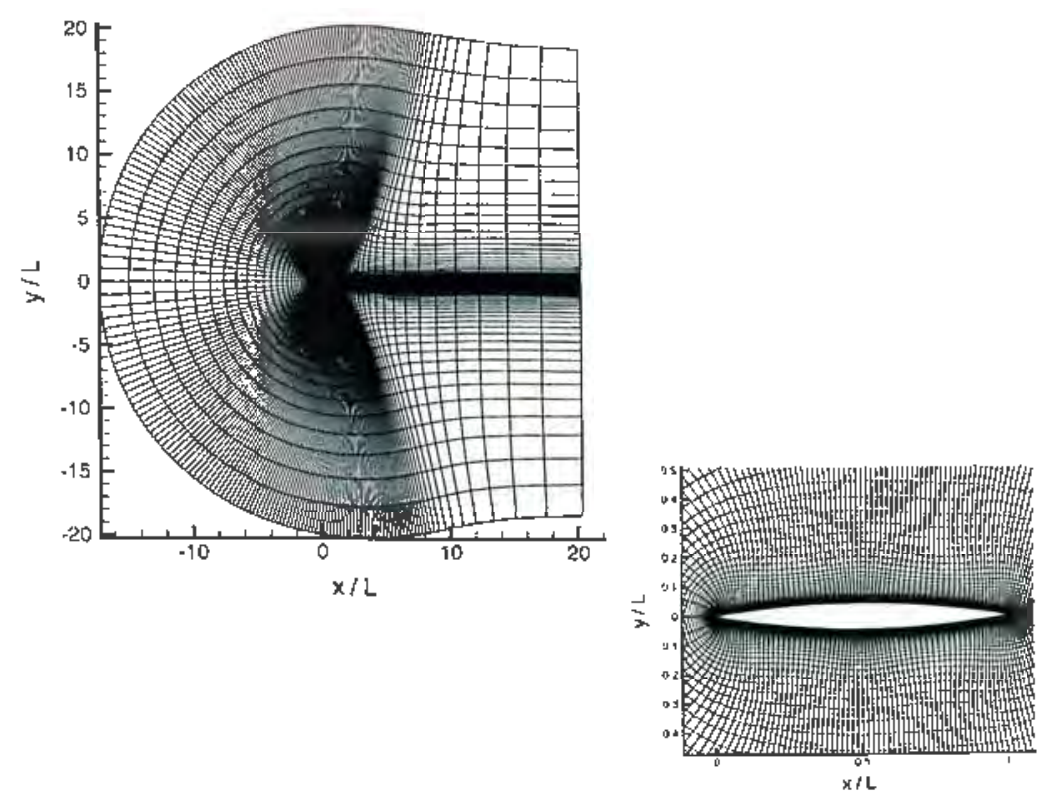

Figure 4.3: Full and close-up view of computational grid for two-dimensional solutitions.

\subsubsection{Two-Dimensional Results}

The local linearization method in two-dimensions was applied to the parabolicarc airfoil described in Eq. 4.38 and the cubic centerline shape. These results are then compared to computational results calculated using the Transonic $\underline{\text { Unsteady }}$ Rotor Navicr-Stokes (TURNS) code ${ }^{71}$. TURNS uses a thin layer approximation to the Navier-Stokes equations, using the Baldwin-Lomax turbulence model ${ }^{72}$ to simulate turbulence. The hyperbolic computational grid used (sec Fig. 4.3) had 217 points in the streamwise wraparound direction (including 74 in the wake region) and 91 points in the normal direction. The grid was oxtended 20 chord lengths in all directions from the surface of the gcometry. All viscous solutions wore solved with a turbulent Rcynold's number of 6 million. The viscous solutions are provided as a worst case scenario for comparison to the inviscid, 


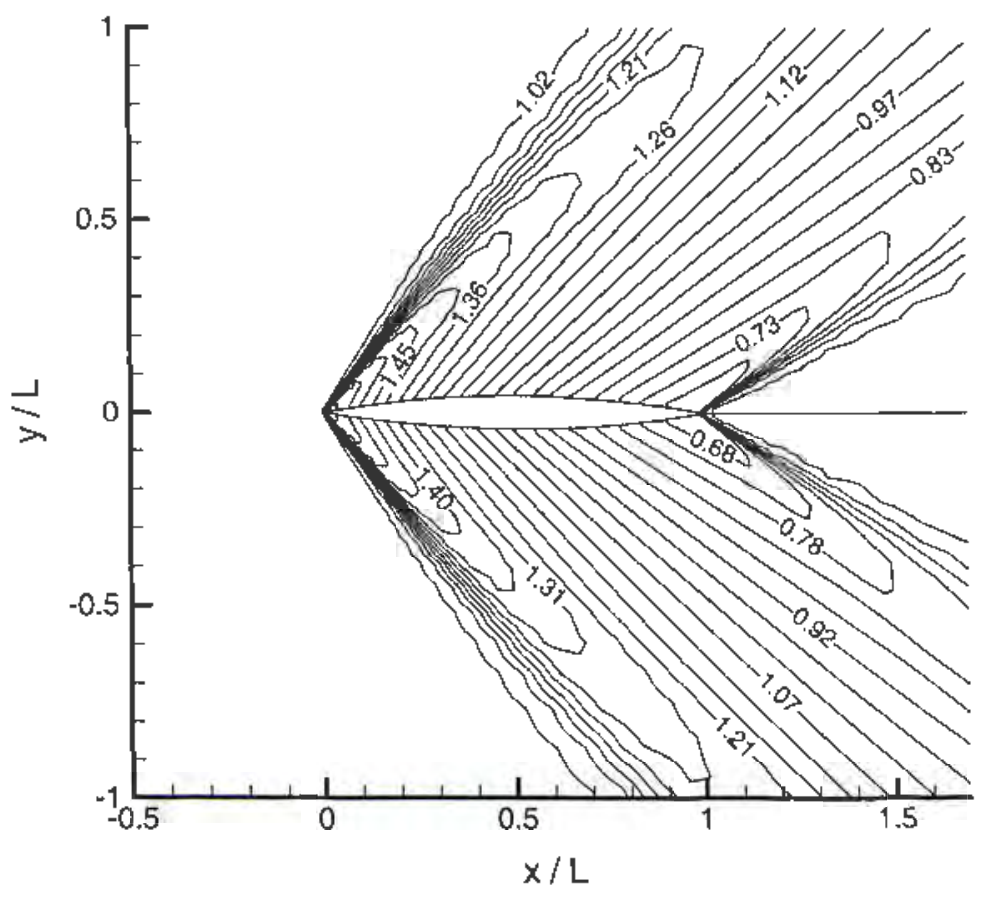

Figure 4.4: Computational supersonic nondimensionalized pressure distribution over parabolic airfoil $\left(M_{\infty}=1.57\right)$.

theorotical results.

Along with the full airfoil profile, for the subsonic and transonic solutions, a half airfoil profile is solved. The half airfoil profile is the same as the full airfoil, except that the top half of the airfoil is cut-off, leaving a flat surface. This geometry was solved numerically to demonstrate the effects of the upper surface on tho lower surface aerodynamics and to emulate the relatively flat surface of the example vehicle geometry.

The supersonic, viscous, computational solution for pressure distribution over the parabolic arc at Mach 1.57 and over the cubic centerline at Mach 1.65 are shown in Figs. 4.4 and 4.5, respectively. These Mach numbers correspond to roughly the upper critical Mach number on the geometries. The parabolic arc 


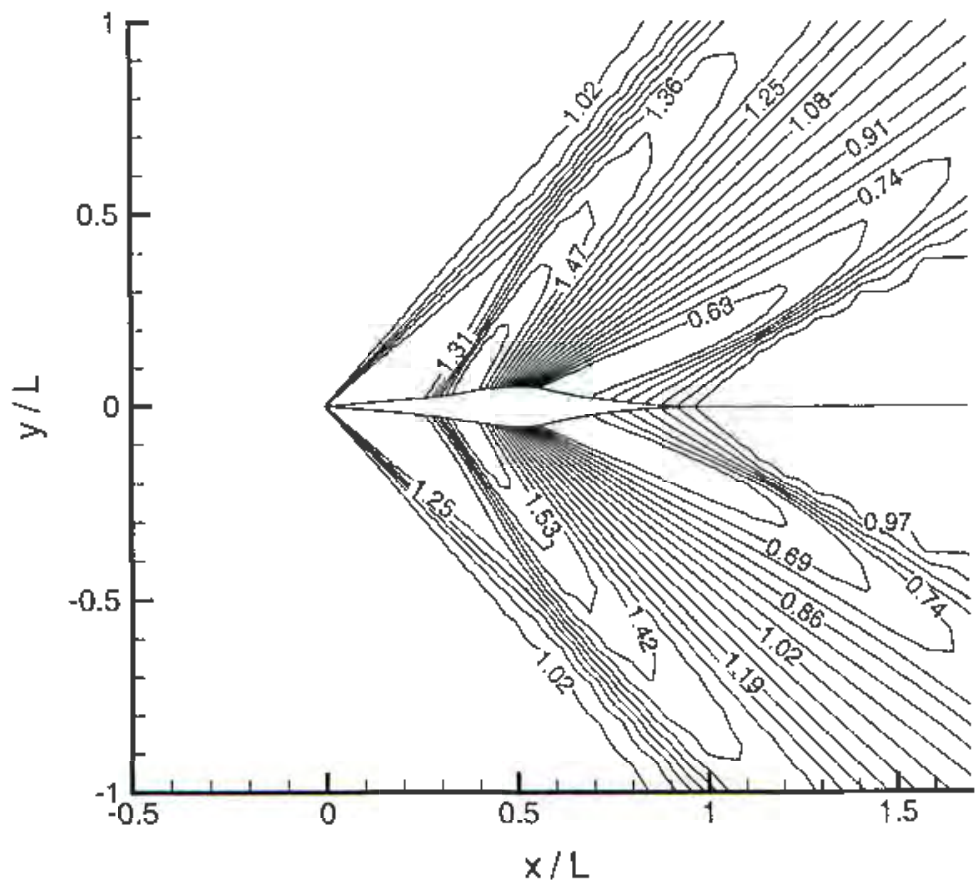

Figure 4.5: Computational supersonic nondimensionalized pressure distribution over cubic airfoil $\left(M_{\infty}=1.65\right)$.

solution (Fig. 4.4) shows leading edge shocks followed by a smooth expansion over the length of the geometry. The flow then ends with a trailing edge shock, followed by a uniform wake region. For the cubic geometry (Fig. 4.5), leading odge shocks are observed, followed by a uniform region (because of the linear portion of the geometry). At the end of the linear section, a second shock forms because of the geometry increasing in cross-scctional area. Following the second shock, the flow expands over the maximum thickness area and then recompresses near the trailing edge. The flow following the trailing edge is uniform, where the compression waves along the rear of the geometry coalesce into a trailing edge shock dowistream of the geometry.

The surface pressure distributions for the supersonic solutions are shown in 


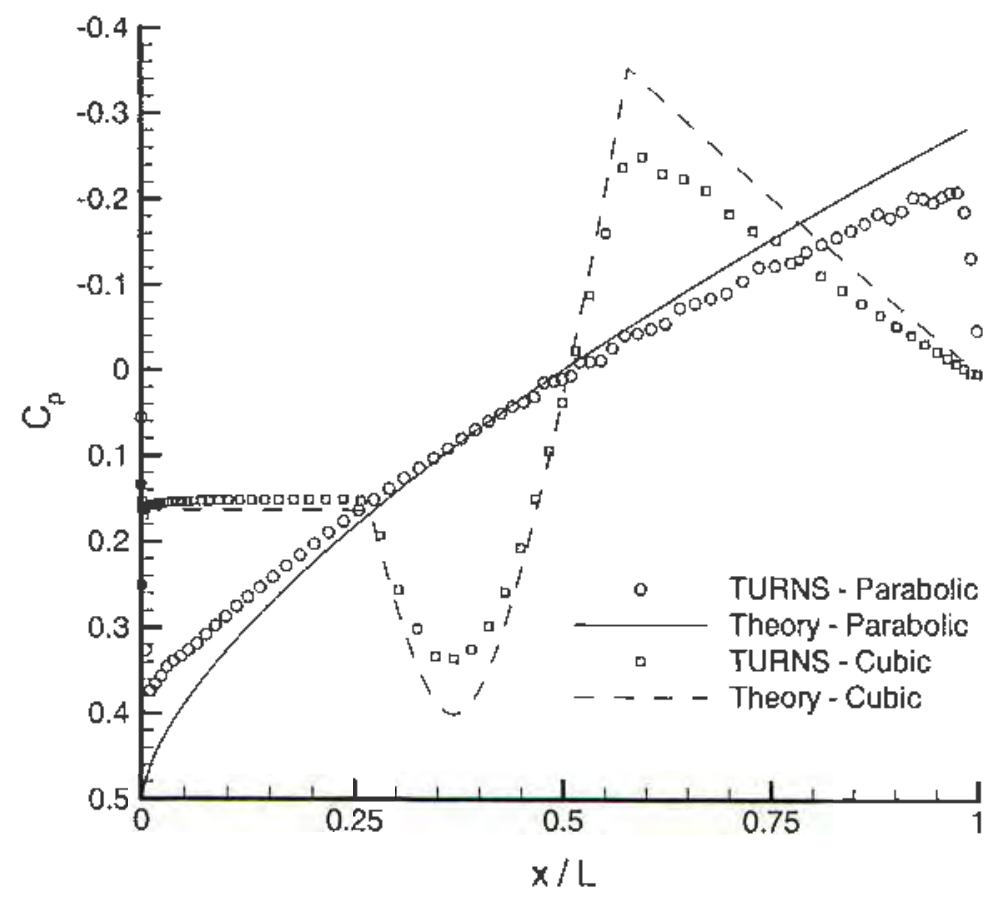

Figure 4.6: Supersonic pressure distribution on parabolic airfoil $\left(M_{\infty}=1.57\right)$ and cubic airfoil $\left(M_{\infty}=1.65\right)$.

Fig. 4.6 for the computational results shown in Figs. 4.4 and 4.5 , where the common airfoil nomenclature of negative pressure coeflicient in the positive $y$ direction is used. Also plotted in Fig. 4.6 are the theoretical results found from Eq. 4.7. For the parabolic are airfoil, the theoretical results deviate from the computational results near the leading and trailing cdges with errors of $23 \%$ and $30 \%$, respectively. For the cubic airfoil, the theoretical results tend to overpredict the peaks in positive and negative pressure coefficient (errors of $19 \%$ and $45 \%$, respectively), with a deviation in the trailing edge prediction. These trends are all bclieved to be from the small disturbance assumptions inherent in the local lincarization method.

Computational solutions for the subsonic, viscous pressure distribution over 


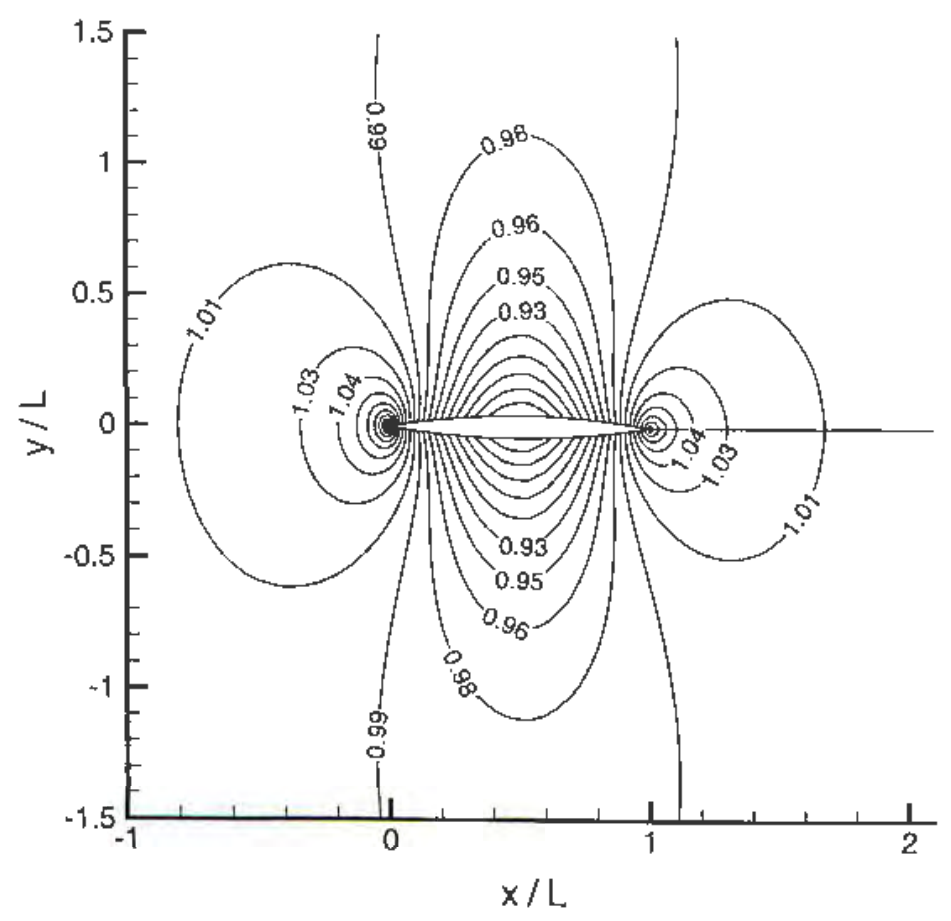

Figure 4.7: Computational subsonic nondimensionalized pressure distribution over full parabolic airfoil $\left(M_{\infty}=0.76\right)$.

the full and half parabolic arc airfoils are shown in Figs. 4.7 and 4.8, respectively. Both airfoils are solved for a freestream Mach number of 0.76 (approximately the lower critical Mach number). For the full airfoil (Fig. 4.7), a symmetric solution is found in the $y$-direction, with a slight asymmetry in the $x$-direction. For the half airfoil solution (Fig. 4.8), the lower surface pressure distribution qualitatively matches the full airfoil solution. Flow near the leading edge and trailing edge are most affected, and the pressure on the lower surface is generally lower in magnitude than the full airfoil solution. These results are not surprising, since subsonic flow behaves elliptically. Hence, the upper surface of the geonetry will aerodynarnically affect the lower surface of the geometry.

Computational solutions for the subsonic pressure distribution over the? full 


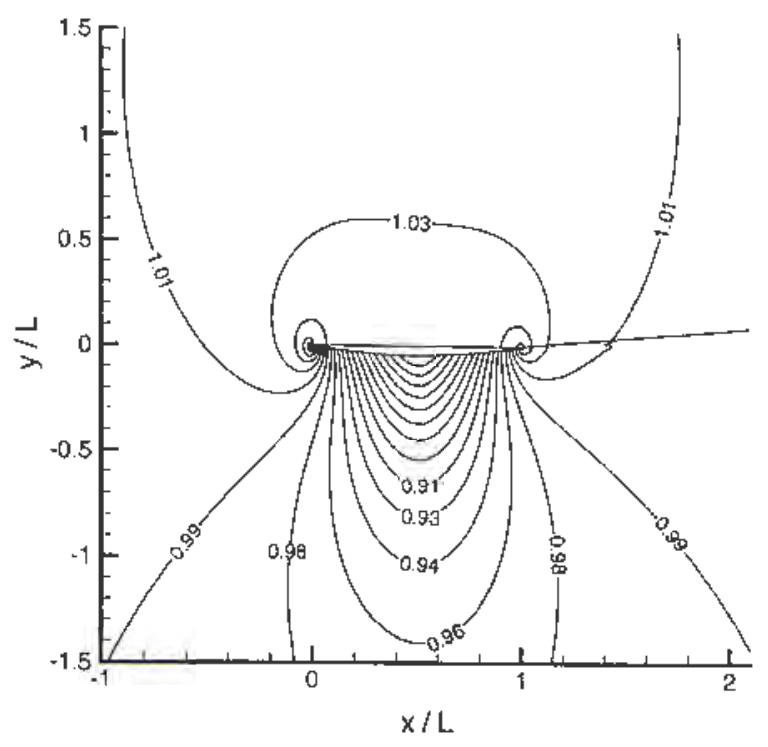

Figure 4.8: Computational subsonic nondimensionalized pressure distribution over half parabolic airfoil $\left(M_{\infty}=0.76\right)$.

and half cubic arc airfoils are shown in Figs. 4.9 and 4.10, respectively. Both airfoils are solved for a frecstream Mach number of 0.65 (approximately the lower critical Mach number). For the full airfoil (Fig. 4.9), a symmetric solution is found in the y-direction, with noticeable differences between it and the parabolic solution (Fig. 4.7). For the half airfoil (Fig. 4.10), the removal of the upper surface again tends to affect the leading and trailing edges the most. However, unlike the parabolic airfoil, the flowfield near the center of the lower surface is much less affected by removing the upper surface than the parabolic case. It is believed that the relatively flat leading and trailing edges of the cubic geometry aid in maintaining the pressure on the lower surface of the geometry compared to the parabolic solution.

The surface pressure distributions for the subsonic solutions are shown in Fig. 4.11 for the computational results shown in Figs. 4.7, 4.8, 4.9, and 4.10. 


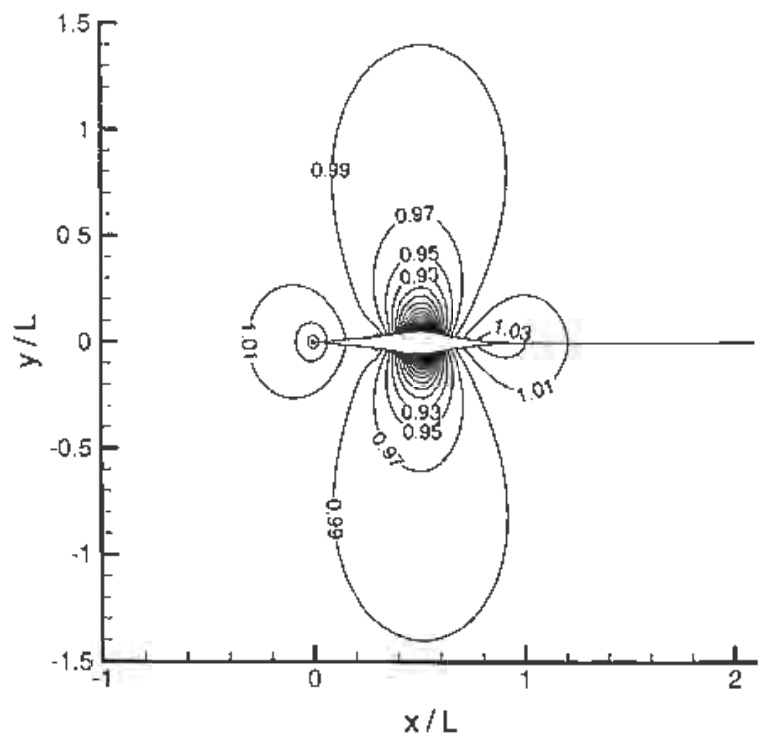

Figure 4.9: Computational subsonic nondimensionalized pressure distribution over full cubic airfoil $\left(M_{\infty}=0.65\right)$.

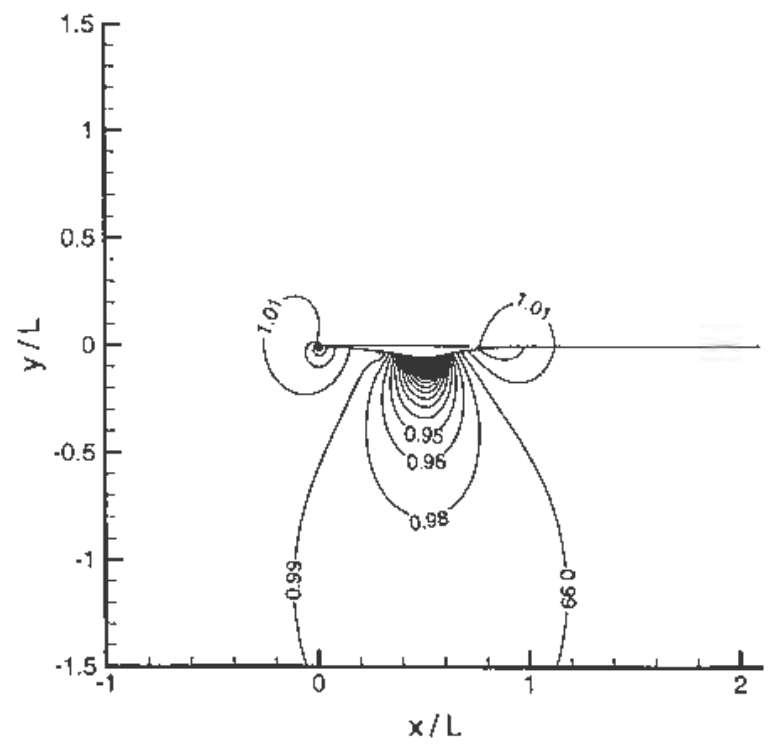

Figure 4.10: Computational sıbsonic nondimensionalized pressure distribution over half cubic airfoil $\left(M_{\infty}=0.65\right)$. 


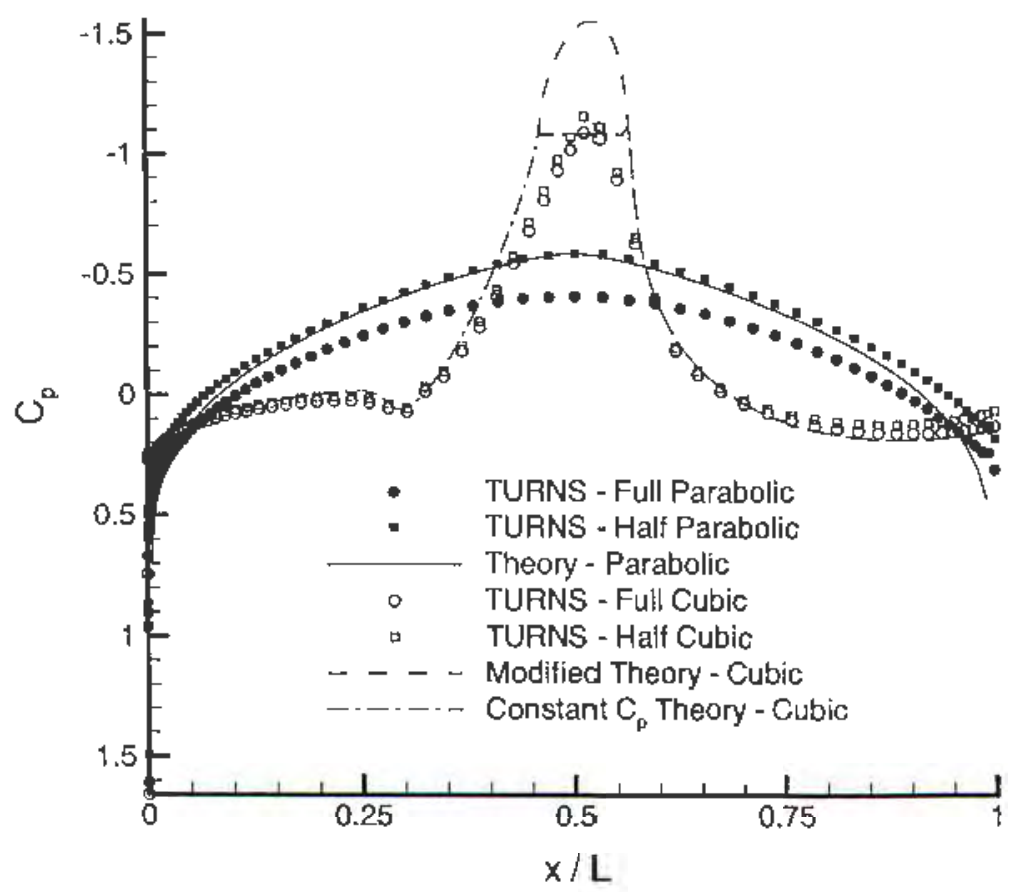

Figure 4.11: Subsonic pressurc distribution on parabolic airfoil $\left(M_{\infty}=0.76\right)$ and cubic airfoil $\left(M_{\infty}=0.65\right)$.

Also plotted in Fig. 4.11 are the theoretical results found from Eq. 4.15 and the constant $C_{p}$ alternative discussed previously, For the parabolic arc, the half airfoil solution is considerably different than the full airfoil shapes, showing the prossure leakage occurring because of the subsonic nature of the flowficid. Note that the half airfoil solution is predicted remarkably well with the local lincarization solution, despite the solution having been derived for full airfoils. For tho cubic airfoil, the half and full airfoil computational solution show much better agreenent, tending to deviate near the trailing edge. The reason for the better agreenont is shown in the comparison of the two profiles in Fig. 4.1. The learling odge of the cubic airfoil has less flow deflection than the parabolic arc airfoil, hence eliminating the upper surface of the cubic airfoil will have less of 
an effect than eliminating the top of the parabolic are airfoil.

The pressire distribution predicted by theory (Eq. 4.15) on the parabolic airfoil gives accurate predictions of the peak negative pressure on the gcometry (crror under $1 \%$ ), but deviates at the leading and trailing edges because of a logarithnic term in the solution of Eq. 4.16. The cubic airfoil has the opposite trond: good predictions at the leding and trailing edges (with a maximum error compared to the full airfoil of under $18 \%$ ), with an overprediction in negative poak pressure in the midsection (55\%). Shown with the solution to Eq. 4.15 is the solution assuming constant $C_{p}$ in the region where the square bracket term of Eq. 4.15 is negative. Although the qualitative trend of pressure is not matched, quantitatively, the constant $C_{p}$ assumption substantially reduces the error in the quantitative prediction of pressure (nax error $40 \%$ ).

Computational solutions for the sonic, viscous pressure distribution over the full and half parabolic arc airfoils are shown in Figs. 4.12 and 4.13, respectively. For tho full airfoil (Fig. 4.12), a symmetric solution is found in the y-direction. Flow is compressed nearing the leading edge, lowering the local Macli numher. After the leading edge, the flow smoothly expands through sonic Mach number and continues to cxpand to the trailing edge. At the trailing edge, a shock wave exists, turning the flow back to frestrean direction. For the half airfoil solution (Fig. 4.13), the flow is again compressed as it flows toward the leading elge. However, unlike the full airfoil solution, the high pressure air on the lower surface expands over the leading edge onto the upper surface. As a result, the flow over the upper surface of the airfoil is entirely supersonic. Also, the expansion at the leading edge tends to lower the leading edge pressure, conpared to the full airfoil solution. Following the leading edge, the flow on the lower surface still resembles the full airfoil solution, showing a small decrease in pressure. Finally, at the trailing cdge, a shock exists turning the flow back to freestream direction.

Computational solutions for the sonic pressure distributions over the full and 


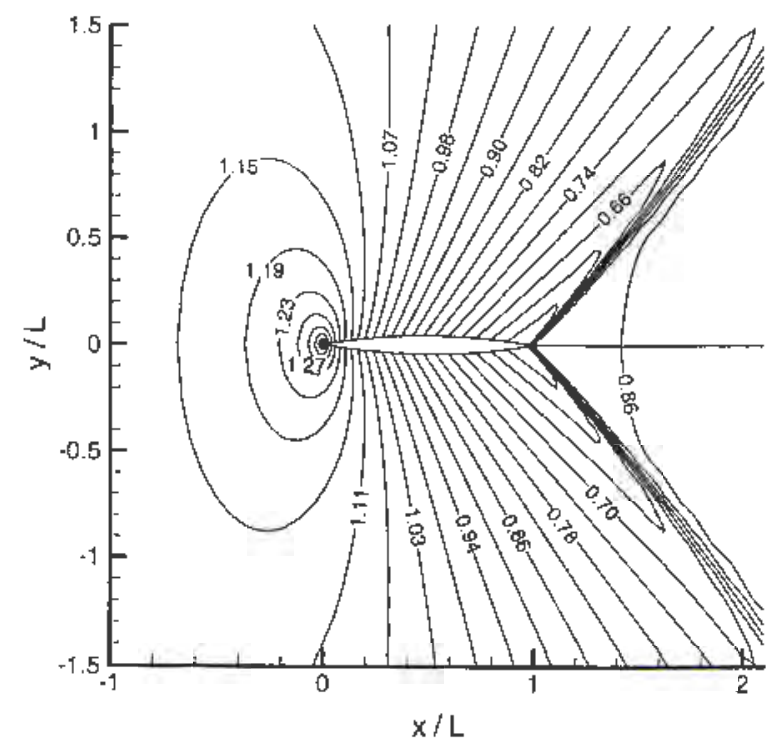

Figure 4.12: Computational sonic nondimensionalized pressure distribution over full parabolic airfoil.

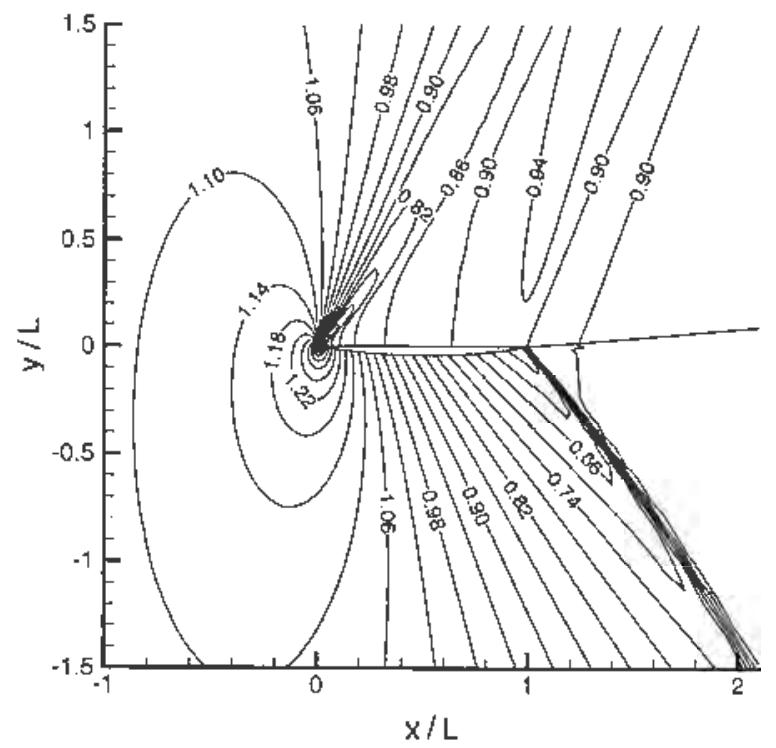

Figure 4.13; Computational sonic nondimensionalized pressure distribution over half parabolic airfoil. 


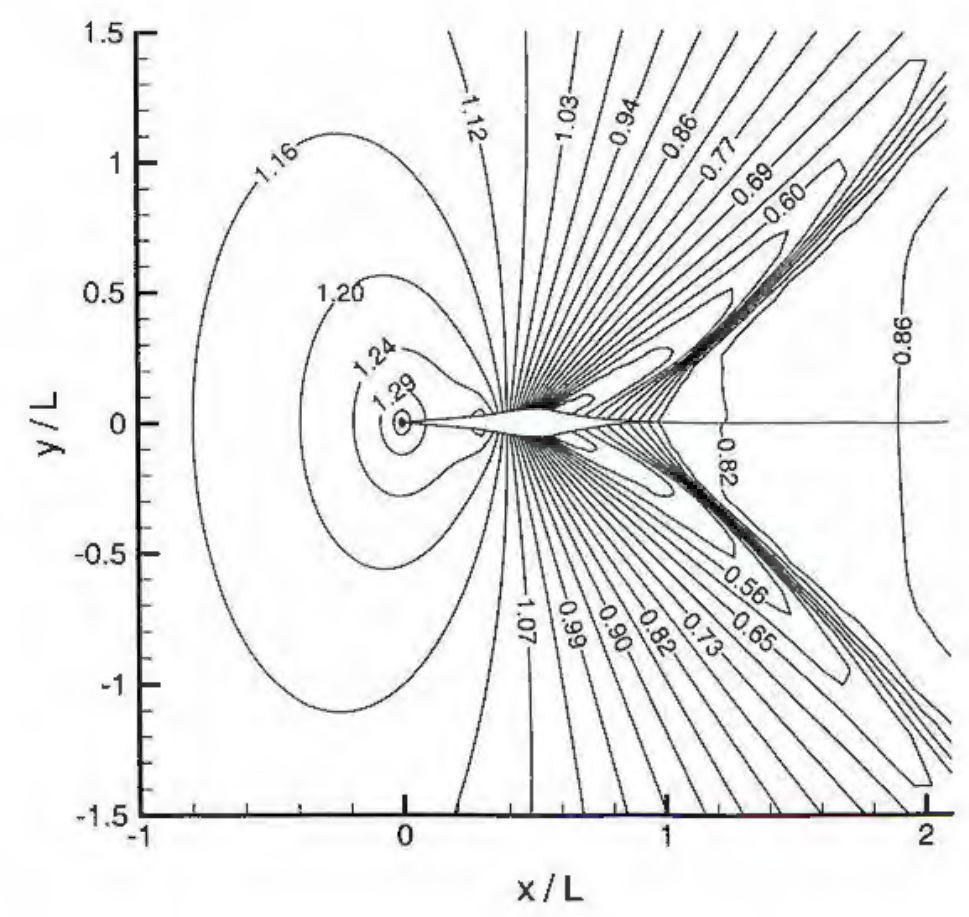

Figure 4.14: Computational sonic nondimensionalized pressure distribution over full cubic airfoil.

half cubic arc airfoils are shown in Figs. 4.14 and 4.15, respectively. For the full airfoil (Fig. 4.14), a symmetric solution is again found in the y-direction. The flow is compressed heading toward the leading edge, followed by a slight compression where the linear portion of the airfoil ends. The flow then smoothly accelerates through sonic Mach number over the maximum thickness location. Following the expansion, the flow is compressed towards the trailing edge. The compression waves from the rear of the airfoil coalesce downstream of the airfoil into a trailing edge shock. For the half airfoil (Fig. 4.15), the same trends are observed at the leading edge as were found with the half parabolic airfoil solution (Fig. 4.13). Following the leading edge, the lower surface flowfield slightly decreases in pressure compared to the full airfoil solution. Near the trailing edge, 


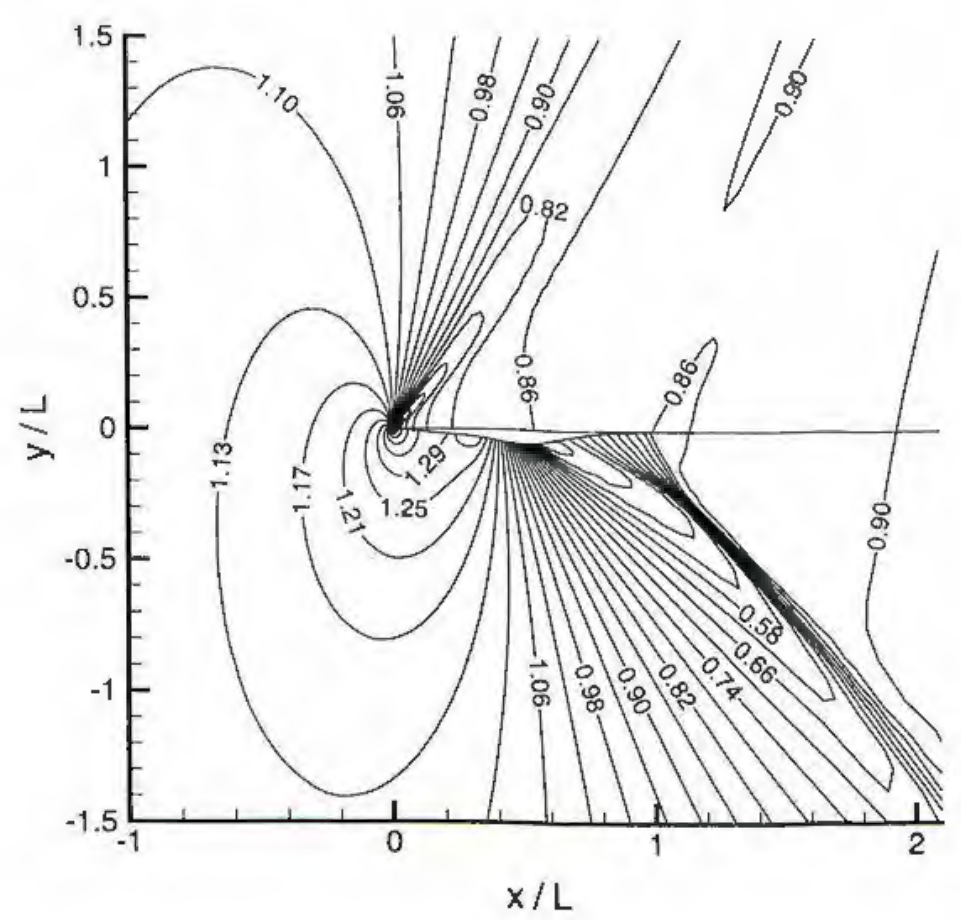

Figure 4.15: Computational sonic nondimensionalized pressure distribution over half cubic airfoil.

the compression waves that turn the flow back to freestream direction coalesce into a shock wave downstream of the airfoil.

The surface pressure distribution for the sonic solutions are shown in Fig. 4.16 for the computational results shown in Figs. 4.12, 4.13, 4.14, and 4.15. Also plotted in Fig. 4.16 are the theoretical results found from Eq. 4.20 as well as experimental results from Michel et al. ${ }^{73}$ for the full parabolic arc airfoil. For the parabolic airfoil, good agreement is found between the theory (Eq. 4.20), the computational results, and the experimental findings with an error in pressure of under $15 \%$ for the majority of the airfoil, excluding the leading edge. The full and half airfoil solutions are quite close (with a difference in $C_{p}$ of under 0.07 for the majority of the airfoil, excluding the leading edge), showing that the lower 


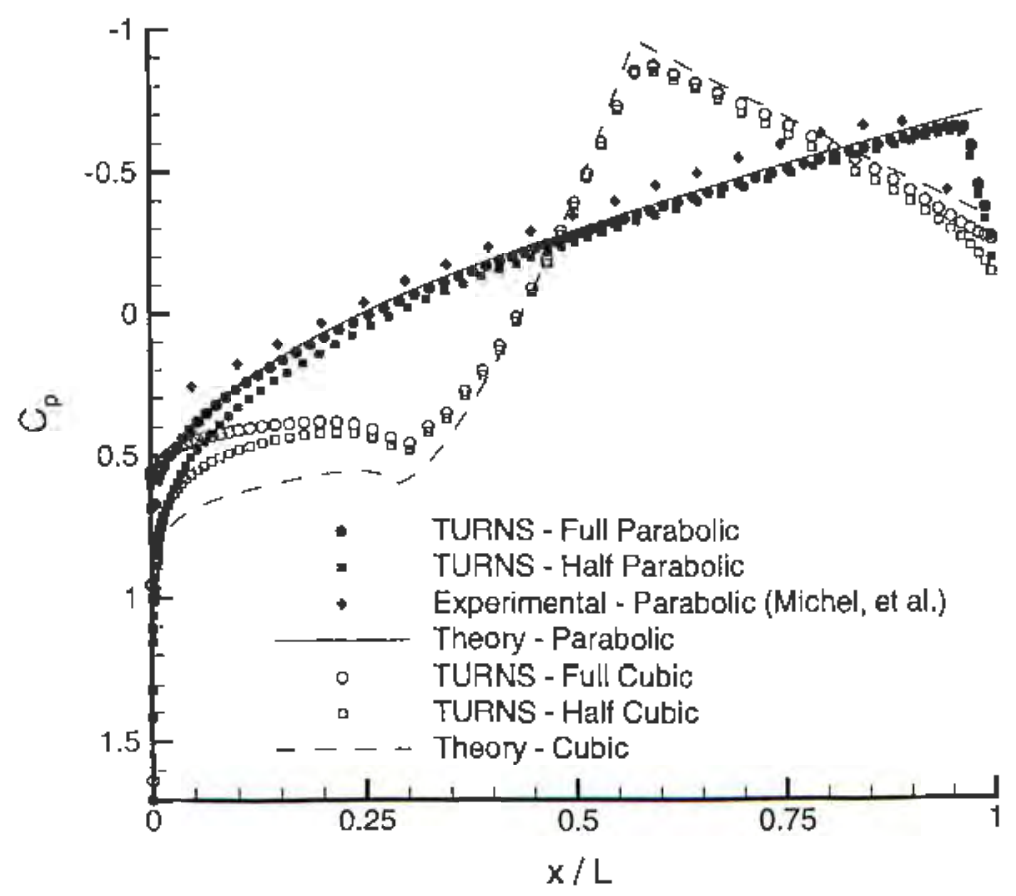

Fignre 4.16: Sonic pressure distribution on parabolic and cubic airfoil.

flowfield is rather immune to communication from the upper surface.

For the cubic airfoil, the intersection point for Eq. 4.22 selected between the sonic and subsonic spline was the end of the linear section of the geonetry. Good agreement is found between the theory and the computational results for the micl and rear sections of the geometry, where the error in peak negative pressure coefficient is under $13 \%$. At the leading edge, Eq 4.22 correctly predicts the qualitative trend of the solution, but overpredicts the pressure by between 25 and $50 \%$ compared to the half and full airfoil solutions. 

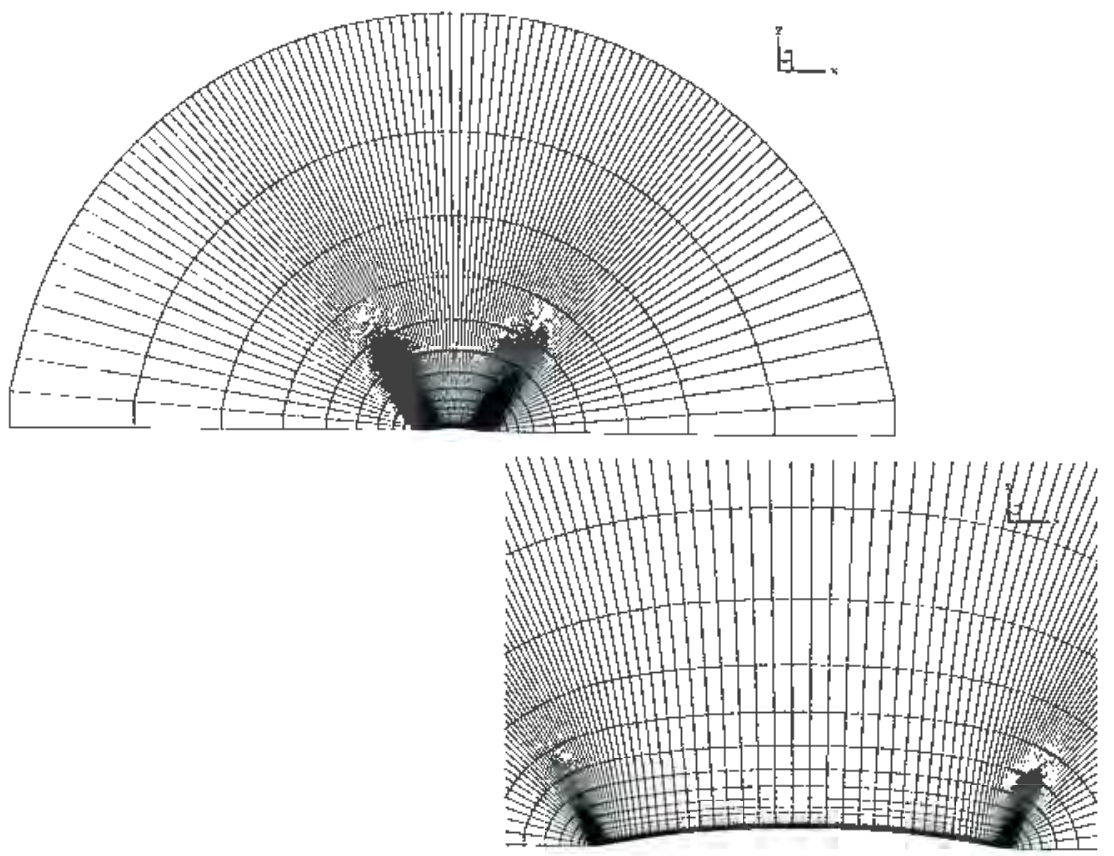

Figure 4.17: Full and close-up viow of computational grid for axisymmetric solutions.

\subsubsection{Axisymmetric Results}

The local linearization method for axisymmetric goometrics was applied to the parabolic-arc airfoil and the cubic centerline shape described above. These results are then compared to computational results calculated using OVERFLOW ${ }^{74}$. OVERFLOW is a thin-layer, Navier-Stokes solver that is capable of integrating multiple, overset grids. The hyperbolic grid (see Fig. 4.17) contained 100 points in the streamwise wraparound direction, 45 points in the direction normal to the surface, and 24 points in the azimuthal direction. The grid was extended a distance of ten vehicle Iengths from the surface of the geometry in all directions. Turbulence was modeled in these computational solutions using the Baldwin-Lomax model ${ }^{72}$ (same as used in the 2-D solutions). All viscous solutions were solved with a turbulent Reynold's number of 6 million (the same as 


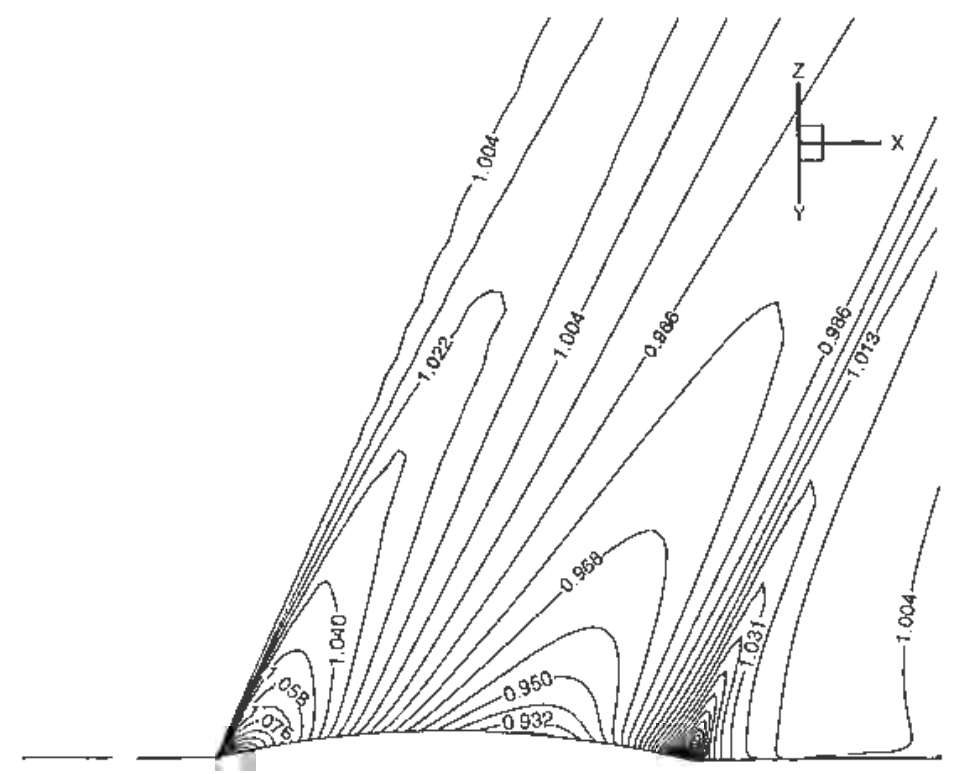

Figure 4.18: Supersonic nondimensionalized pressure distribution over parabolic body of revolution $\left(M_{\infty}=1.21\right)$.

the 2-D solutions). Additionally, experimental data on the parabolic-are profilers are provided for the subsonic and sonic solutions.

The supersonic, viscous, computational solution for pressure distribution over the parabolic and cubic bodies of revolution at Mach 1.21 are shown in Figs. 4.18 and 4.19, respectively. These Mach numbers correspond to roughly the ruper critical Mach number on tho goometries. The parabolic body of revolution shows the same general fow pattern as the two-dimensional solution: leading edge shock, smooth expansion over the longth of the body, trailing cdge shock to return the flow to the freestrcam direction. The cubic body of revolution has the same basic flow pattern as the two-dimensional solution, but the compressions occurring are noticably woaker (shocks arc weak).

The surface pressure distribution for the supersonic, axisymmetric solutions are shown in Fig. 4.20 for the computational results shown in Figs. 4.18 and 4.19. 


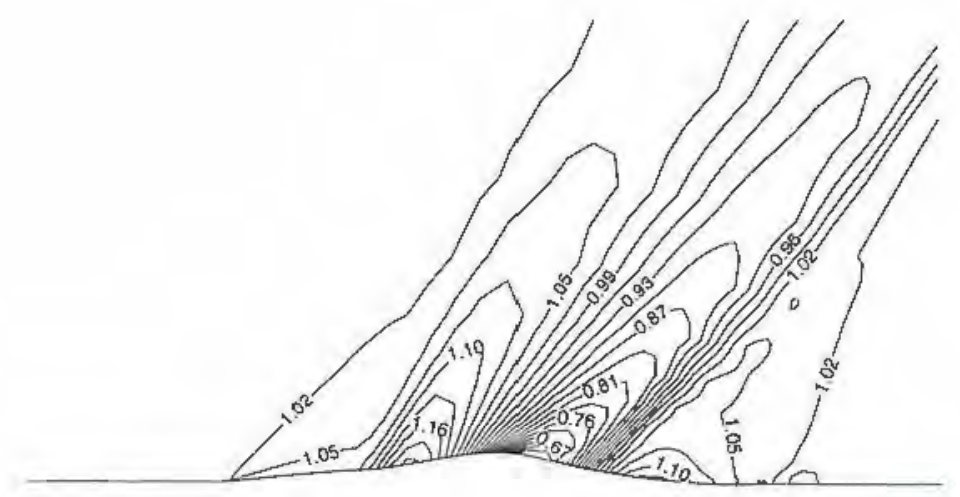

Figure 4.19: Supersonic pressure distribution over cubic body of revolution $\left(M_{\infty}=1.21\right)$.

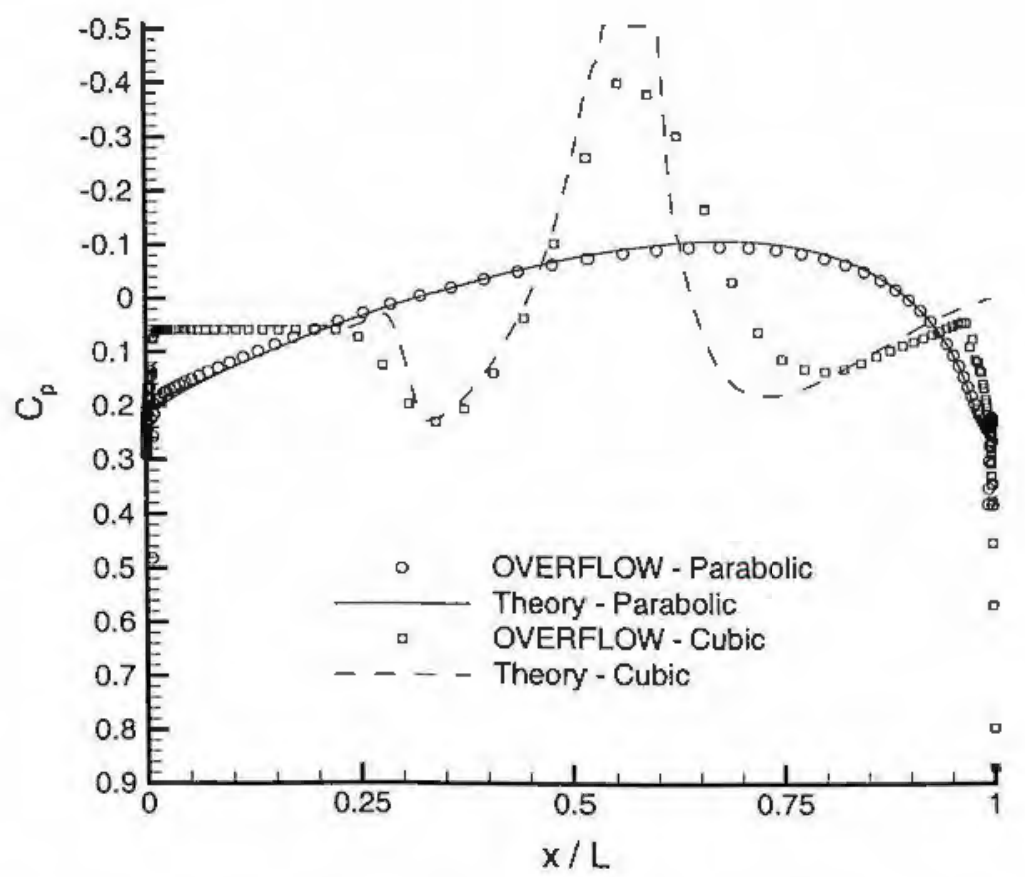

Figure 4.20: Pressure distribution on parabolic and cubic body of revolution $\left(M_{\infty}=1.21\right)$. 
Also plotted in Fig. 4.20 are the theoretical results found from numerical integration of Eq. 4.29 (labeled "theory"). For the parabolic body of revolution, excellent agreement was found between the theory and computational solution, with a difference in peak negative pressure coefficient of 0.01 and an error at the leading edge of around $10 \%$.

The solution of the cubic geometry is split into two parts. The front portion of the geometry is solved by assuming that the pressure coefficient at the leading edge is equivalent to the pressure on the surface of a cone. The solution is then numerically integrated from the leading edge to the midpoint of the seventh-order spline between the first and second cubic sections by use of Eq. 4.29. The solution of the rear of the geometry is found from assuming that the pressure coefficient is zero at the trailing edge. The solution is then numerically integrated forward to the midpoint of the seventh-order spline between the first and second cubic sections using Eq. 4.29. A limit on minimum pressure coefficient was assumed by isentropically expanding the flow from freestream to an angle corresponding to the maximum in negative slope on the geometry using 2-D Prandtl-Meyer flow. The limit in pressure coefficient using this method was approximately -0.5 for the case shown in Fig. 4.20.

Figure 4.20 shows that the theoretical solution for the cubic geometry matches well with the computational results at the leading and trailing edges, with a $C_{p}$ difference of 0.007 at the leading edge and a difference in $C_{p}$ of 0.07 at the trailing edge. The largest error occurs in the region where the two cubic sections intersect (corresponding to the region of minimum pressure coefficient). The error in peak negative pressure coefficient was around $30 \%$. The solution was found to pass the assumed pressure coefficient limit in this area, and overpredicts the pressure immediately beyond the intersection by over a factor of 10 . This error is largely attributed to the geometry to be solved. As stated earlier, the original geometry was designed to have continuous slope, not continuous third 


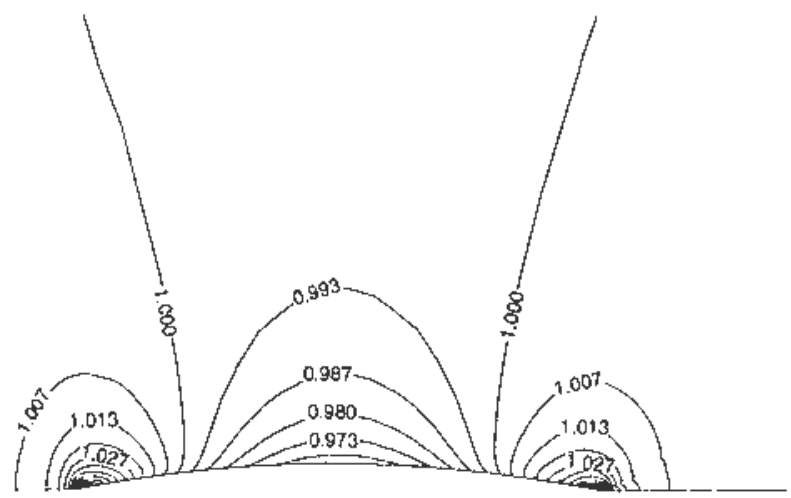

Figure 4.21: Subsonic nondimensionalized pressure distribution over parabolic hody of revolution $\left(M_{\infty}=0.8\right)$.

derivatives. Hence, the third derivative in cross-sectional area is nowhere near the theory's requirement of a smooth geometry. Although the seventh-order spline helps to make the shape continuous through the third derivative, wide variation in the triphe derivative is olserved. It is believed that in order to use this theory to its full potential, the geometry of the vehicle should be such that the third derivative in cross-sectional area is smooth. However, considering the limitations of the theory, and the assumptions involved in finding the solution, the theory does an acceptable job of modeling the flow.

The sulssonic, viscous, computational solution for pressure distribution over the parabolic and cubic bodies of revolution at Mach 0.8 arc shown in Figs. 4.21 and 4.22, respectively. These Mach numbers correspond to roughly the lower critical Mach number on the geometries. Both solutions show the same flow fortures as the two-dimensional solutions, with the exception of a pressure relieving effect due to axisymmetry.

The surface pressure distribution for the suhsonic, axisymmetric solutions are shown in Fig. 4.23 for the computational results slown in Figs. 4.21 and 4.22. 


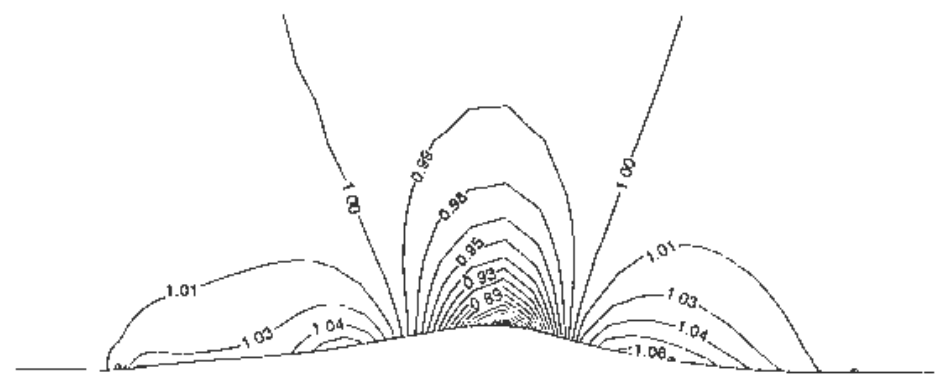

Figure 4.22: Subsonic pressure distribution over cubic body of revolution $\left(M_{\infty}=\right.$ $0.8)$.

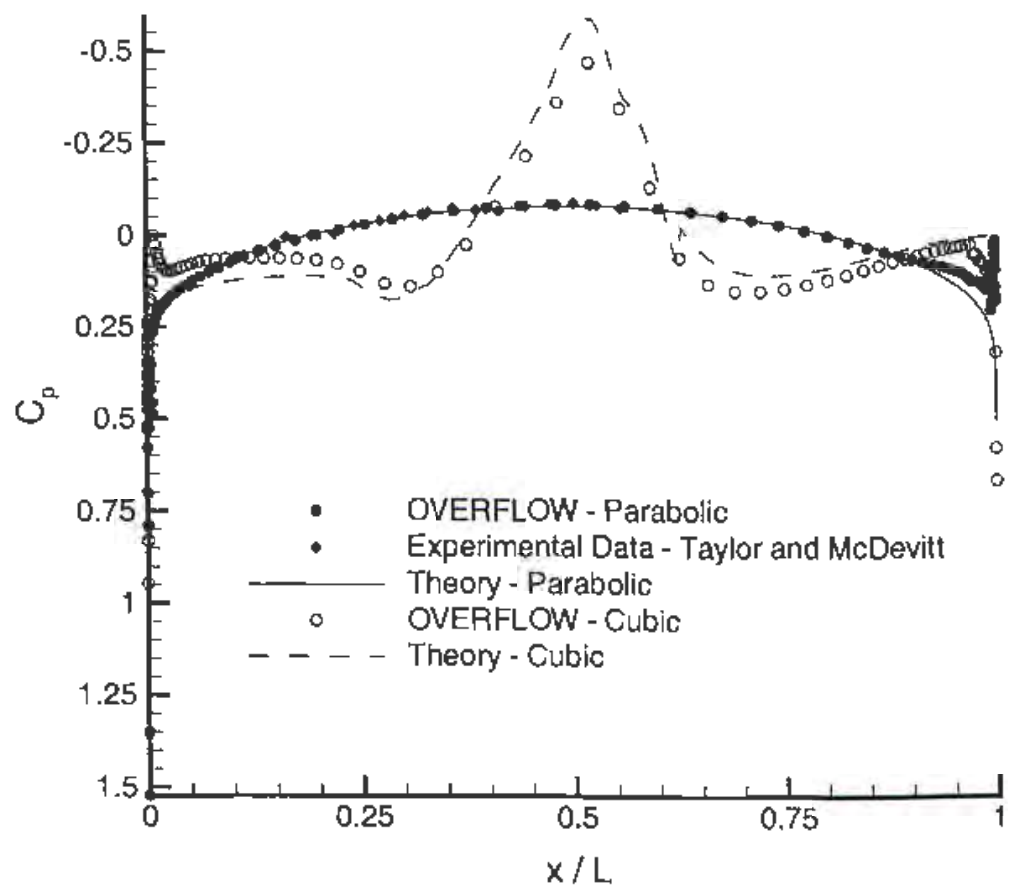

Figure 4.23: Pressure distribution on parabolic and cubic body of revolution $\left(M_{\infty}=0.8\right)$. 
Also plotted in Fig. 4.23 are the theoretical results found from numerical integration of Eq. 4.34 (labeled "theory") and experimental data from Taylor and McDevitt ${ }^{75}$ for a parabolic arc body. For the parabolic body, excellent agreement is found in comparing both the computational and experimental results, with a difference in peak negative pressure coefficient of 0.0075 , an error of under $6 \%$ in pressure coefficient near the leading edge, and an error in pressure coefficient of around $50 \%$ near the trailing edge (where flow separation affects the computational solution).

The solution of the cubic geometry is split into three parts for the subsonic solution. The starting point found from the $S^{\prime \prime}=0$ location is used to numerically integrate Eq. 4.34 to the leading edge and to numerically integrate to the end of the second seventh-order spline. For the rear portion of the shape, the pressure coefficient is assumed to be zero at the trailing edge, and Eq. 4.34 is integrated backwards to the intersection of the second cubic section and the seventh-order spline. At this intersection point, a small discontinuity in pressure coefficient exists. The solution, in general, is in good agreement with the computational results, with an error along the leading edge of a factor of 2 , and an error in the peak negative pressure region of about $27 \%$. Note that as freestream Mach number decreases, the theoretical solution to the peak negative pressure region shows more error in comparison to the computational results. This is a direct consequence from the analytical geometry first assumed, and may possibly be mitigated with a smoother shape.

The sonic, viscous, computational solution for pressure distribution over the parabolic and cubic bodies of revolution are shown in Figs. 4.24 and 4.25, respectively. A noticeable difference between the two-dimensional solutions and the axisymmetric solutions is the movement of the rearward shock from the trailing edge on the two-dimensional solutions to a point on the body for the axisymmetric solutions. The location of shock waves on the surface of the geome- 


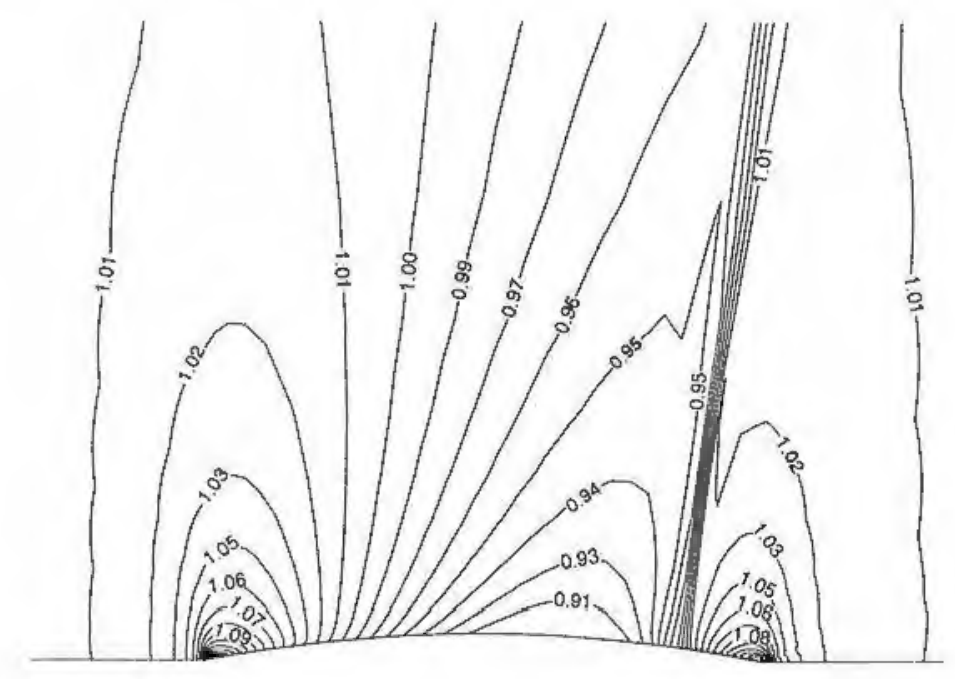

Figure 4.24: Sonic nondimensionalized pressure distribution over parabolic body of revolution.

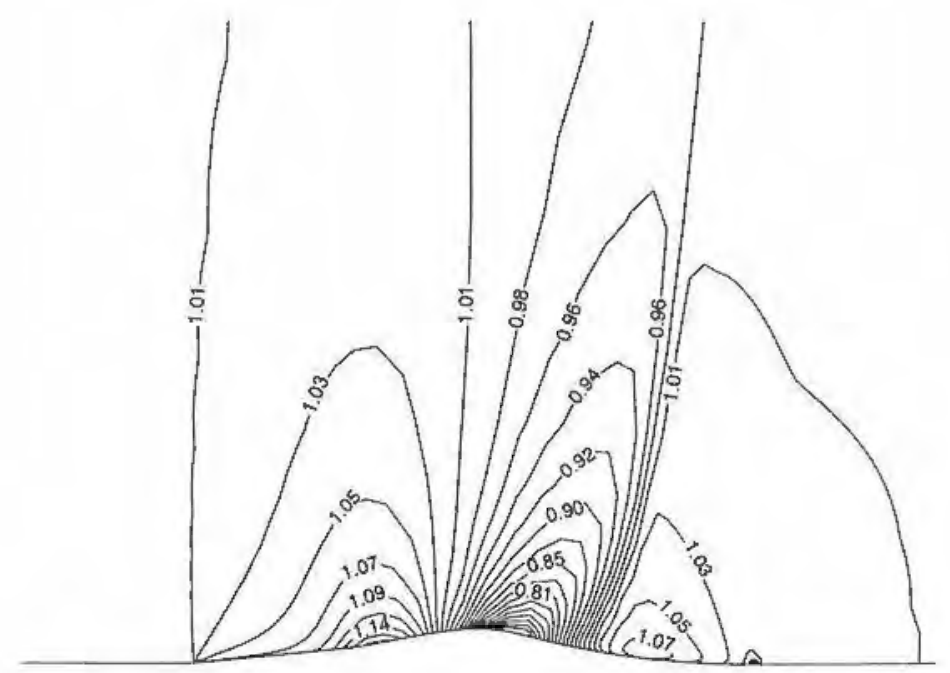

Figure 4.25: Sonic nondimensionalized pressure distribution over cubic body of revolution. 


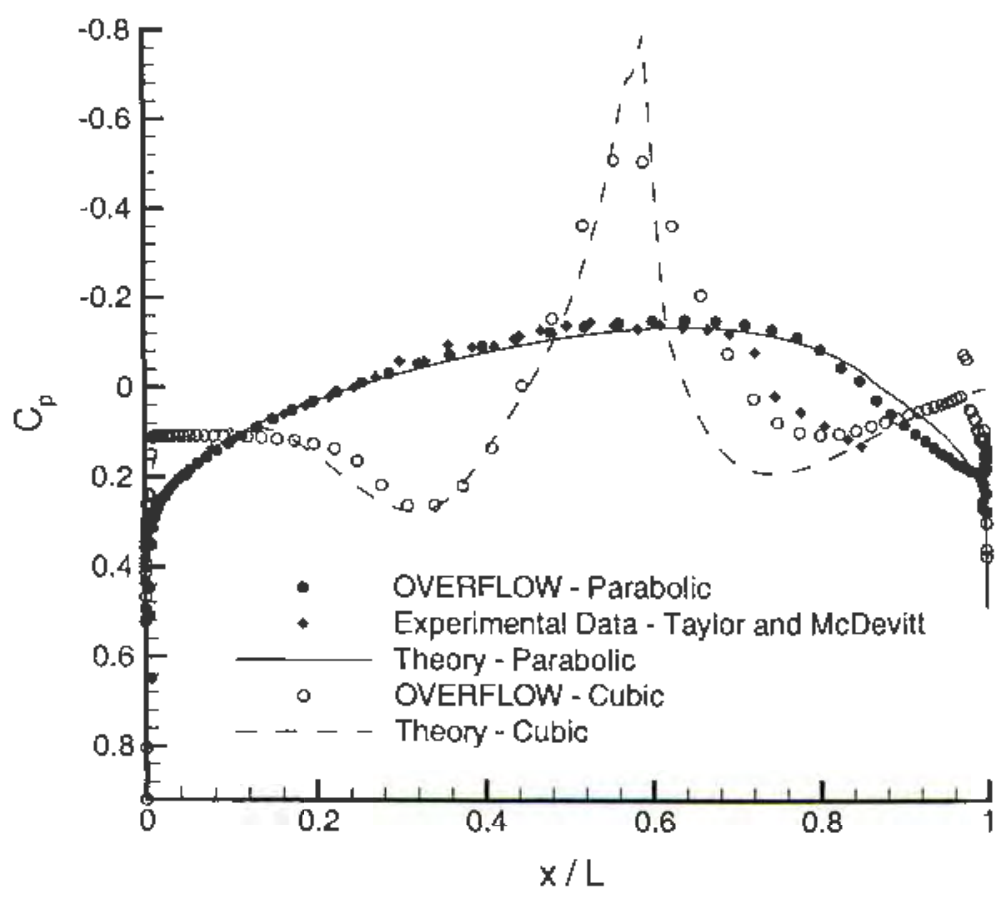

Figure 4.26: Pressure distribution on parabolic and cubic body of revolution at sonic velocity.

tries will almost certainly result in boundary-layer separation, with an inherent riso in drag.

The surface pressure distribution for the sonic, axisymmetric solutions are shown in Fig. 4.26 for the computational results shown in Figs. 4.24 and 4.25 . Also plotted in Fig. 4.26 are the theoretical results found from numerical intcgration of Eq. 4.37 (labeled "thcory") and experimental data from Taylor and McDevitt ${ }^{75}$ for a parabolic arc body. Again, for the parabolic body, good agrecment is found between the theory, the computational solution, and the experimental results, with an error in peak negative pressure coefficient of around $10 \%$. The largest discrepancy between the results is found on the rear portion of the object, where shock / boundary layer interactions are causing flow separation. 
The sudden rise in pressure in the experimental results is due to the presence of a sting to hold the geometry in the wind tunnel. This effect is not modeled in the computational results nor the theory presented.

It was noted in Spreiter's discussion of the sonic axisymmetric solutions that the solution in the nose region will have a large error due to the subsonic nature of the flow ${ }^{69}$. This error was encountered while directly integrating Eq. 4.37 for the cubic geometry. To better model the leading edge of the geometry, the solution of $\mathrm{Eq}$. 4.37 for a sonic cone was used to model the leading enge prossures

$$
C_{p}=\frac{2\left(M_{\infty}^{2}-1\right)}{M_{\infty}^{2}(\gamma+1)}-2 \tau^{2} \ln \frac{\tau x}{l_{c}}-\tau^{2}+\tau^{2} \ln \left[\tau^{2}+\frac{4\left[1-\left(\frac{x}{l_{c}}\right)^{2}\right]}{M_{\infty}^{2}(\gamma+1) \tau^{2} e^{C}}\right]
$$

whore $l_{c}$ refers to the length of the conical nose section. The solution of Eq. 4.39 goes to positive infinity as $x$ goes to zero and negative infinity as $x$ goes to $l_{c}$ due to the logarithmic terms. Hence, $\mathrm{Eq} .4 .39$ was taken from near the leading edge to the point of maximum slope of $C_{p}$ (found from taking the derivative with respect to $x$ of $\mathrm{Eq}$. 4.39). The forward portion of the cubic geometry was solved by selecting the starting point where $S^{\prime \prime}=0$ and integrating toward the leading edge to the point of rraximum slope and integrating toward the trailing edge to the point of minimum slope (it is noted that when the exponential term in Eq. 4.37 is positive, the supersonic, axisymmetric solution is solved to the point of minimum slope). These points correspond to the region where smooth, expanding flow exists that is best modeled by Eq. 4.37 .

The solution to the leading edge region and the sonic solution are then connocted using a cubic spline for a smooth pressure profile. The solution on the rear of the geometry is again solved by assuming that the pressure coeflicient at the trailing edge is zcro and then integrating the supersonic solution (Eq. 4.29) to the point of minimum slope. A minimum pressure limit predicted by Prandtl-Meyer flow is imposed to lower solution error.

The result of the above nothodology for solving the sonic flow over the cubic 
geometry is labeled "theory" in Fig. 4.26. The forward portion of the geomctry is predicted well (crror under $20 \%$ ), with the largest error occurring near the maximum and minimum slope regions ( $35 \%$ and $55 \%$, respectively). The rearward portion of the geometry demonstrates the same tendencies as seen in Fig. 4.20. The maximum error occurs in the region where the triple derivative in cross-sectional area has the largest deviations (errors over a factor of 10 ). Again, this error is attributable to the genmetry selected and may be mitigated by selecting a smoother solution.

\subsection{Application to Full Vehicle Geometry}

Solution of the vehicle geometry commences by eliminating the engine midline of the vehicle (see Fig. 2.1). The midline (where the compression ramps and engine are located) was eliminated to simplify the solution. Eliminating the engine section will result in an crror in the pressure distribution due to interference effects, but is considered negligible for this investigation. Where the engine section used to be, the bottom fusclage is extended along the span direction to the centerline of the geometry. The resulting geometry is shown in Fig. 4.27.

The flowfield on the vehicle is solved by taking longitudinal slices in the freestream direction of the vehicle (see Fig. 4.27), and then solving the $2-\mathrm{D}$ and axisymmetric pressure distributions on each slice. After the vehiche has been solved assuming 2-D and axisymmetric flow, the solutions are combined together. These solutions are then compared to an inviscid CFD solution over the 3-D, full vehicle (bottorn surface replaced with the geometry in Fig. 4.27) for three Mach numbers: $0.65,1$, and 1.65. The subsonic and supersonic Mach numbers are the sub- and supercritical Mach numbers on the 2-D geometry. It is assumed that for the subsonic and supersonic solutions there is no transition through Mach 1. 


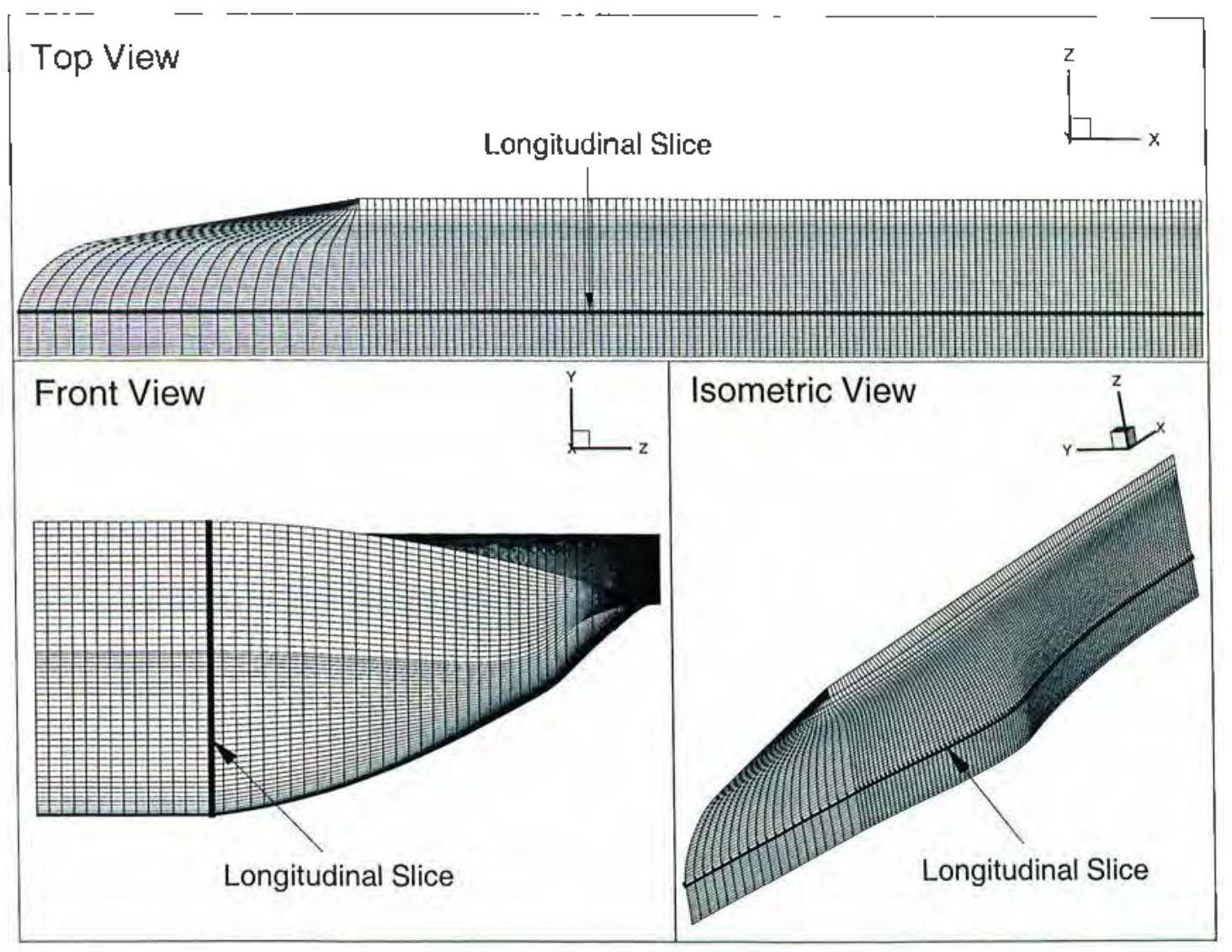

Figure 4.27: Bottom surface geometry and longitudinal slice used in solving vehicle pressure distribution. 
The inviscid theory is compared to an inviscid, 3-D CFD solution for validation; thus, viscous effects (which will be significant) are not modeled nor validated. Also, this method does not account for cross-flow (i.e., flow in the spanwise direction), nor has it been applied to anything other than zero angle of attack (except for supersonic solutions to be discussed in a later section).

The 3-D, inviscid, full vehicle CFD solution was calculated using OVERFLOW $^{74}$. Symmetric boundary conditions were used to solve the flowfield over half of the vehicle. OVERGRID ${ }^{76}$ was used to generate an overset mesh about the 3-D object. The computational grids consisted of an inner hyperbolic grid (see Fig. 4.28) generated about the geometry and an outer box grid (see Fig. 4.29) generated to extend the far field boundary. A first-order interpolation is used to transfer conservative variable values between grid blocks. Conservative variables are transferred every ten iterations and remain frozen for the other iterations. The restriction of variable transfers eases memory requirements and increases computational performance, with a small decrease in convergence rate. The inner hyperbolic grid had dimensions of 46 points in the spanwise wraparound direction, 279 points in the streamwise wraparound direction (including 104 points in the wake region), and 39 points in the direction normal to the surface. The inner grid was extended to a distance of one vehicle length from the surface of the geometry. The outer box grid had dimensions of $65 \times 61 \times 32$ points and was extended to a distance of over 10 vehicle lengths from the surface of the geometry.

A comparison between the centerline, supersonic, computational pressure distribution on the lower surface of the full vehicle with the 2-D and axisymmetric computational pressure distribution is shown in Fig. 4.30 for a Mach number of 1.65. At the leading edge, the pressure on the full vehicle is initially $2-\mathrm{D}$ and then linearly decreases to the axisymmetric solution by the end of the linear portion of the vehicle. This behavior is analogous to a 3-D shock wave, where properties 


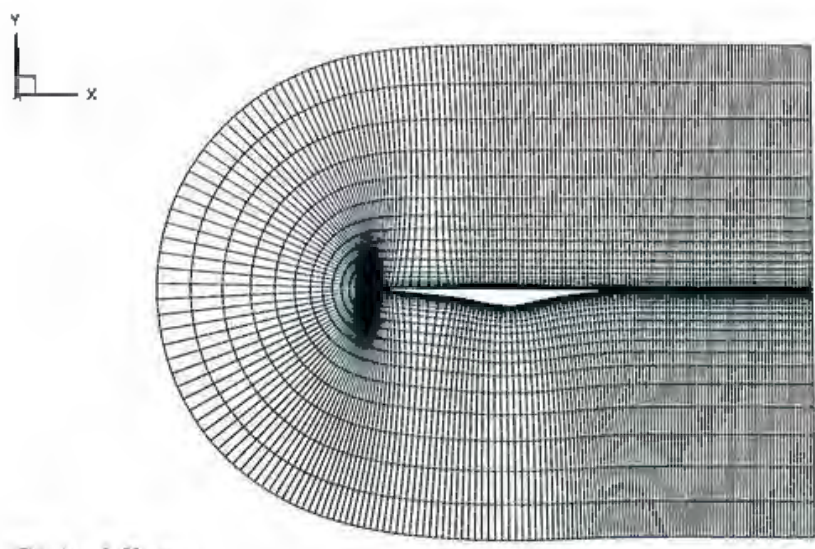

Side View
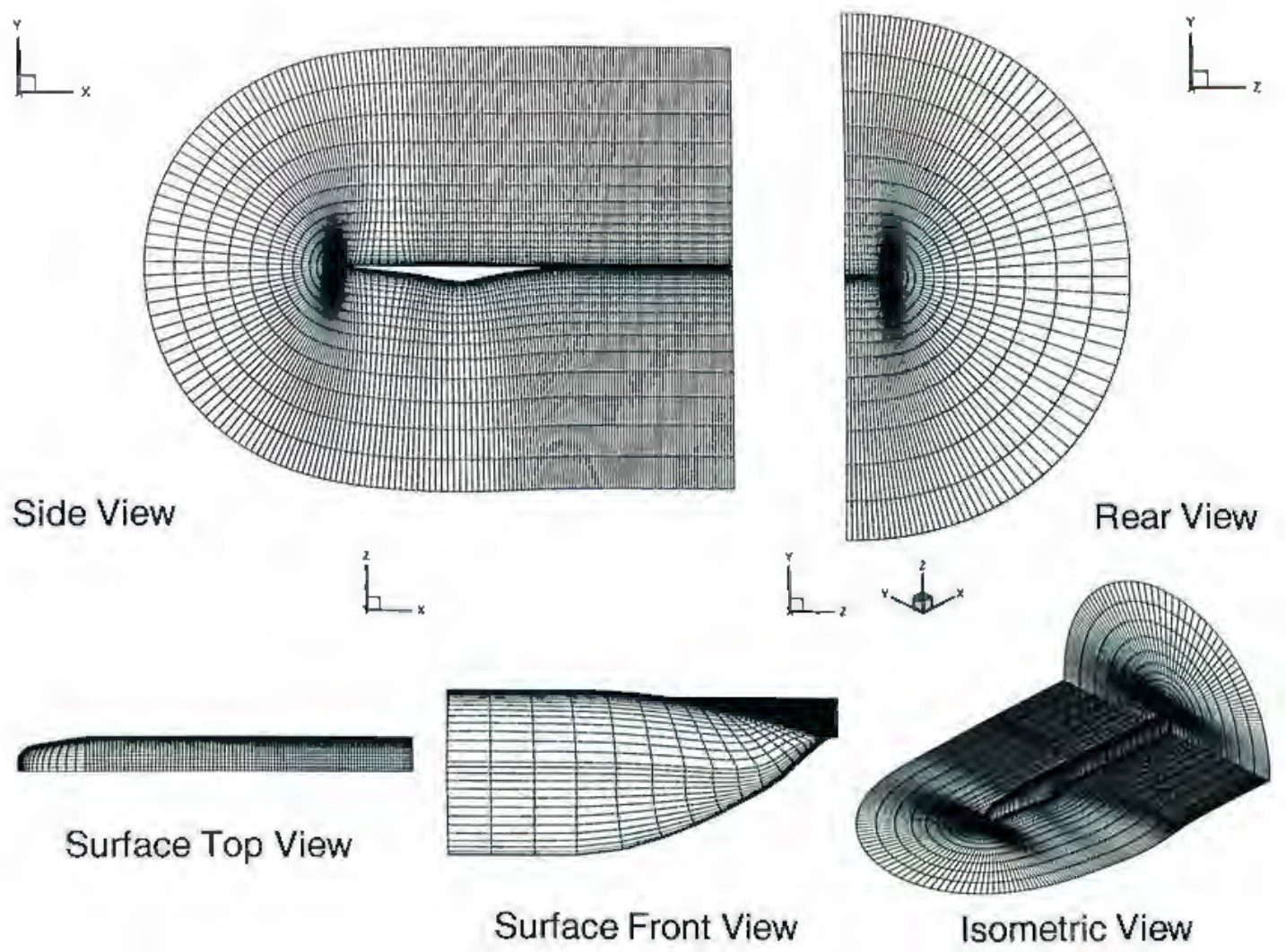

Figure 4.28: Inner lyyperbolic grid used for computational solutions (including surface grid). 


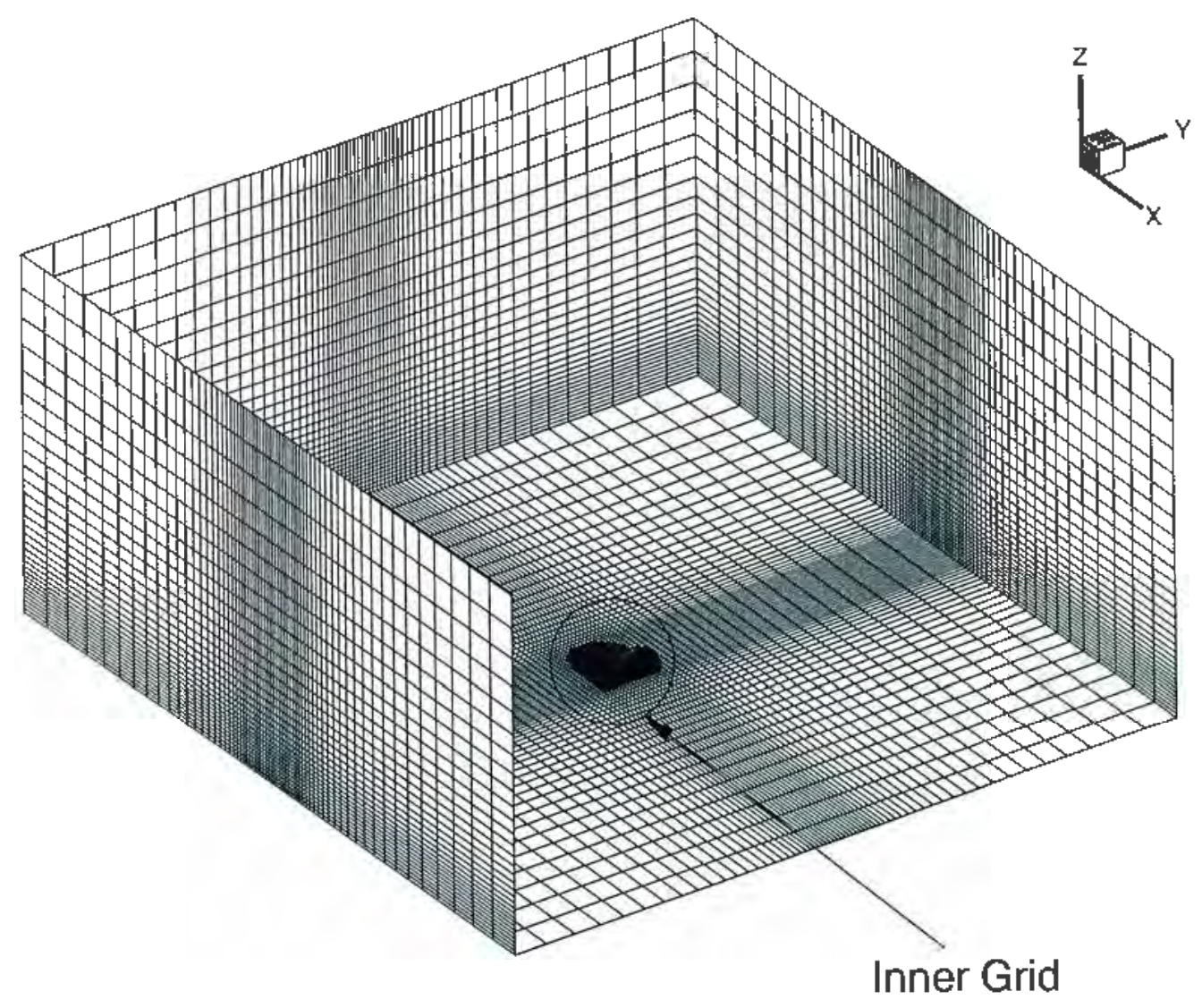

Figure 4.29: Outer box grid (with inner hyperbolic grid) used for computational solutions. 


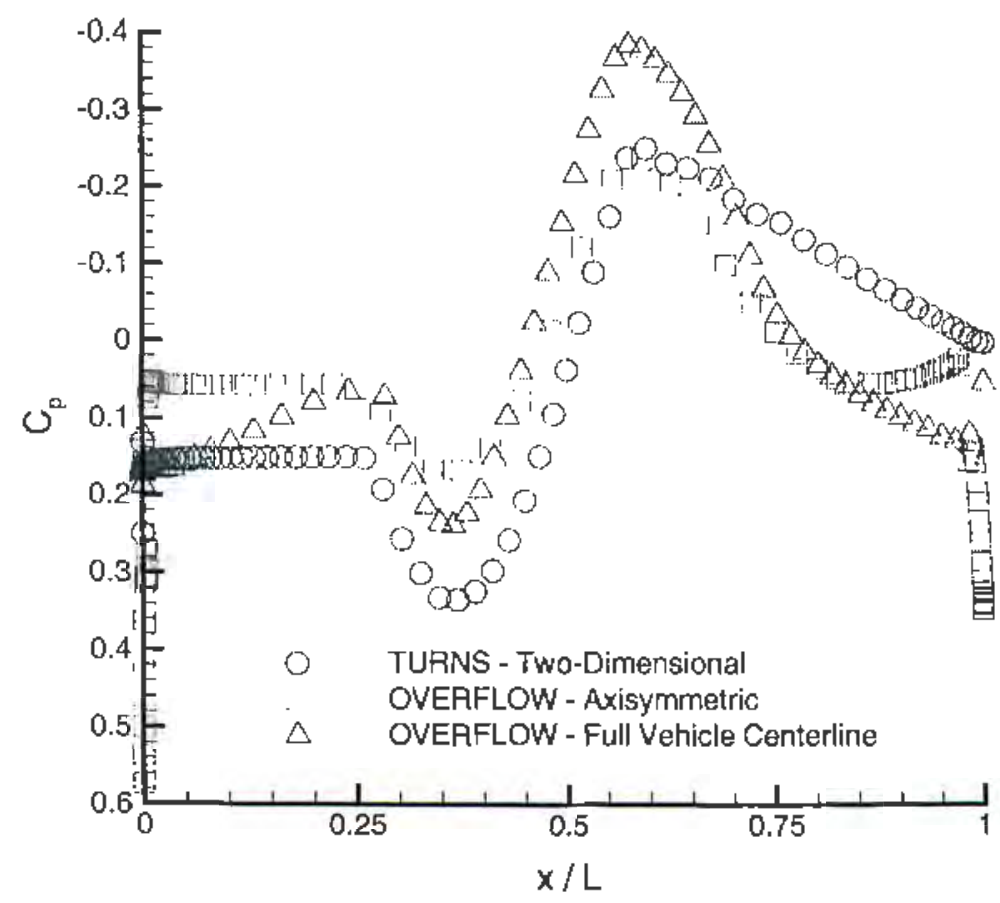

Figure 4.30: Comparison of supersonic CFD results $\left(M_{\infty}=1.65\right)$.

immediatoly behind the shock arc 2-D and then transition to 3-D away from the shock. Following the linear leading edge, the prossurc distribution follows the qualitative trend of the $2-\mathrm{D}$ solution to the point of minimum slope. The solution then transitions from 2-D to axisymmetric, intersecting the axisymmetric solution at about the $3 / 4$ point on the body. After this point, the solution then follows the qualitative trend of the 2-D solution to the trailing edge.

A comparison between tife centerline, subsonic, computational pressure distribution on the lower surface of the full vehicle with the 2-D and axisymmetric computational pressure distribution is shown in Fig. 4.31 for a Mach number of 0.65 . Overall, the pressure distribution on the full vehicle follows the axisynnetric solution more closely than the $2-\mathrm{D}$ solution. The leading edge region again looks to transition from $2-\mathrm{D}$ to axisymmetric in the linear region. Following the 


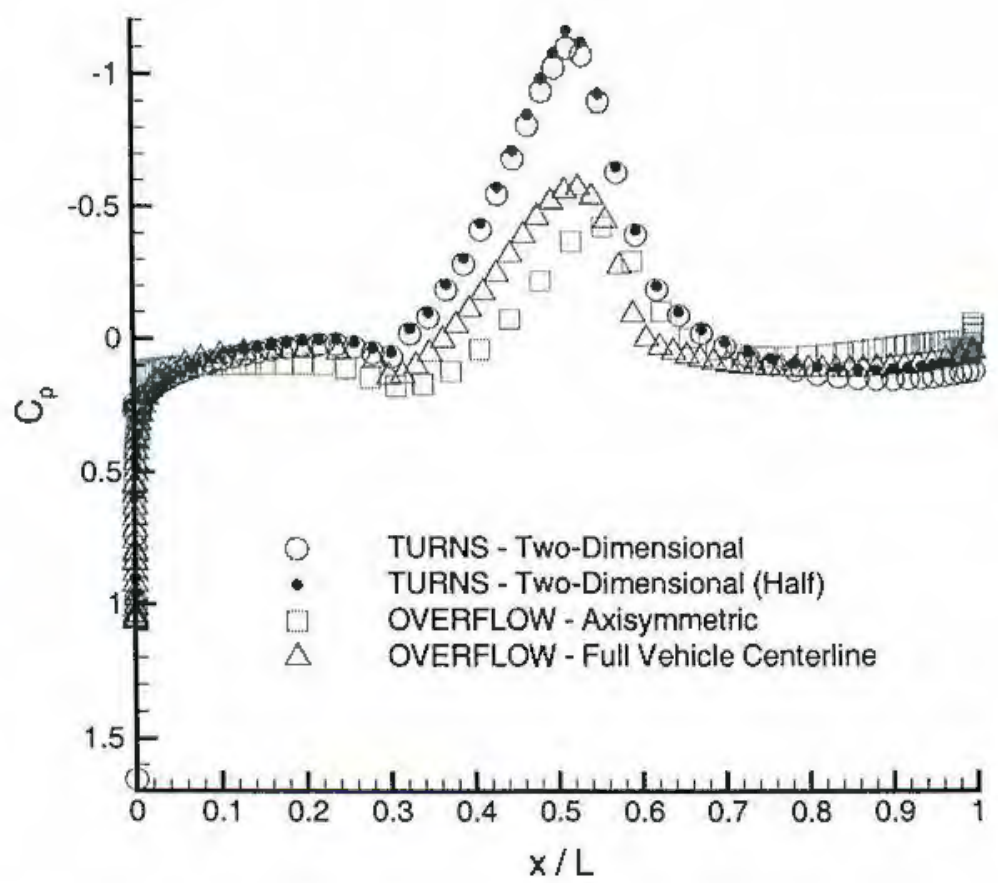

Figure 4.31: Comparison of subsonic CFD results $\left(M_{\infty}=0.65\right)$.

maximum slope point, the computational solution is bracketed by the 2-D and axisymmetric solution. At the minimum slope point, the full vehicle solution behaves like the axisymmetric geometry to around the $3 / 4$ point on the body, where the solution is bracketed by the 2-D and axisymmetric solutions to the trailing edge. Qualitatively, averaging the 2-D and axisymmetric solutions gives a good approximation to the full vehicle profile.

A comparison between the centerline, sonic, computational pressure distribution on the lower surface of the full vehicle with the 2-D and axisymmetric computational pressure distribution is shown in Fig. 4.32. The full vehicle profile behaves like the axisymmetric solution from the leading edge to the maximum slope point. The solution then transitions to the 2-D solution, which predicts the peak negative pressure coefficient very well. Following the minimum slope 


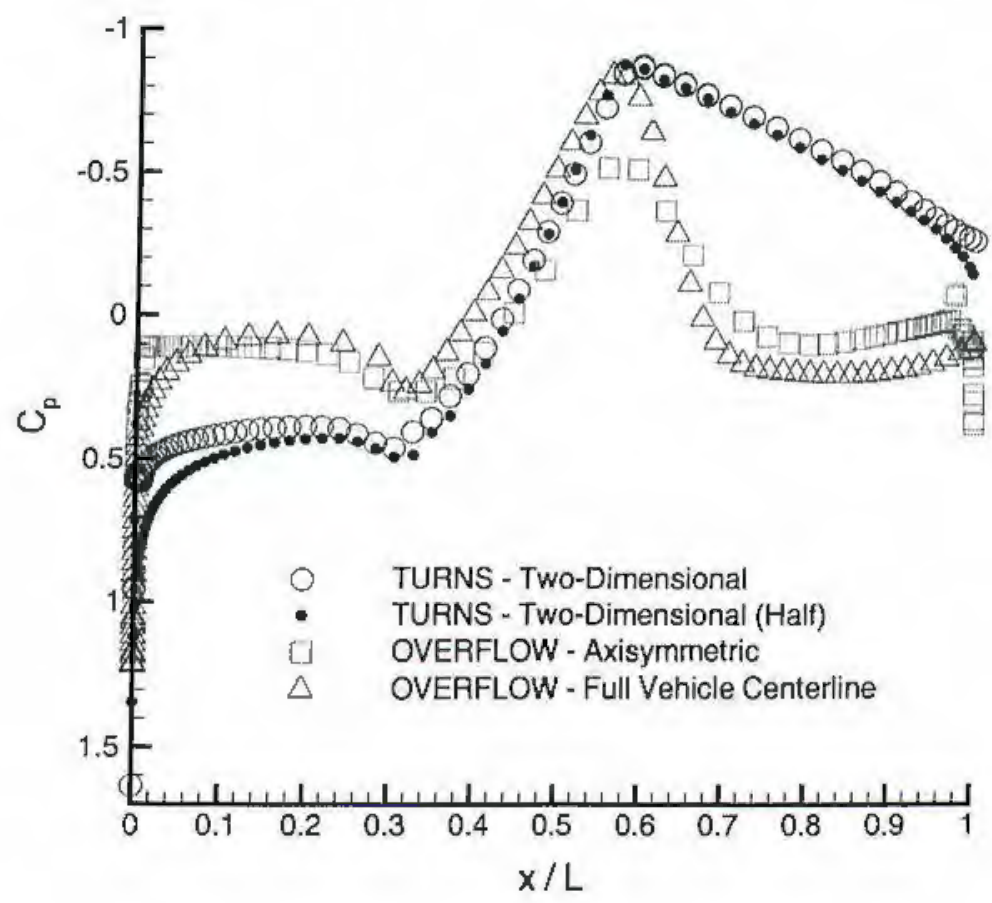

Figure 4.32: Comparison of sonic CFD results.

point, the solution transitions back to the axisymmetric solution to the trailing edge.

Ultimately, it would be desirable to combine the 2-D and the axisymmetric solutions together to predict the pressure profile on the full vehicle. The simplest way of combining the two solutions would be an average of the two results at each solution point on the geometry. A more rigorous way of blending the two solutions together would be to determine where on the vehicle the flow would behave more in a 2-D sense and where on the vehicle the flow would behave more in an axisymmetric sense. With this in mind, a proposed combination of the results based on local surface curvature would be

$$
C_{p_{s \mid \mathrm{p}}}=C_{p_{\mathrm{axi}}}\left(\frac{h_{\max }}{R_{\mathrm{loc}}}\right)^{n}+C_{p_{2 D}}\left[1-\left(\frac{h_{\max }}{R_{\mathrm{loc}}}\right)^{n}\right]
$$


where $n$ is an exponential value to be determined, $h_{\max }$ is the maximum height of the geometry, and $R_{\text {loc }}$ is the local radius of curvature. Another way of combining the solutions together, similar to Eq. 4.40, would be

$$
C_{p_{\mathrm{sp}}}=4 \pi C_{p_{\mathrm{axi}}}\left(\frac{S}{\tilde{C}^{2}}\right)^{n}+C_{p_{2 \mathrm{D}}}\left[1-4 \pi\left(\frac{S}{\tilde{C}^{2}}\right)^{n}\right]
$$

where $\tilde{C}$ is the circumference of the cross-section. A blending may also be applied prior to integration of the derivative of perturbation velocity

$$
\left.\frac{d \bar{u}}{d x}\right|_{\mathrm{sp}}=\left.\frac{d \bar{u}}{d x}\right|_{\text {axi }}\left(\frac{h_{\max }}{R_{\mathrm{loc}}}\right)^{n}+\left.\frac{d \bar{u}}{d x}\right|_{2 \mathrm{D}}\left[1-\left(\frac{h_{\max }}{R_{\mathrm{loc}}}\right)^{n}\right]
$$

In all of the methods presented, the base of $n$ is chosen to go from zero for purely 2-D flow to one for purely axisymmetric flow. Finally, a combination may be assembled from directly observing the behavior of the CFD solutions presented in Figs. 4.30 - 4.32. For all of the proposed methods (except the averaging), the determination of the proper method will be largely dependent on the vehicle geometry. It is most likely that no single combination exists that will accurately predict the full vehicle pressure profile for a wide range of geometries. However, the blending may be applicable for geometries that are similar to one another, such as waverider-based vehicle geometries.

\subsection{Validation}

The results of applying the $2-\mathrm{D}$ and axisymmetric solutions to the full vehicle geometry by taking longitudinal slices will now be discussed. On the lower surface, four analytical solutions will be compared (depending on Mach number) with the inviscid, 3-D, full vehicle computational solution: a solution assuming that each slice on the vehicle behaves in a 2-D manner (labeled "2-D"), a solution assuming that each slice on the vehicle behaves axisymmetrically (labeled "Axi"), an average of the 2-D and axisymmetric solutions (labeled "Avg"), and the 


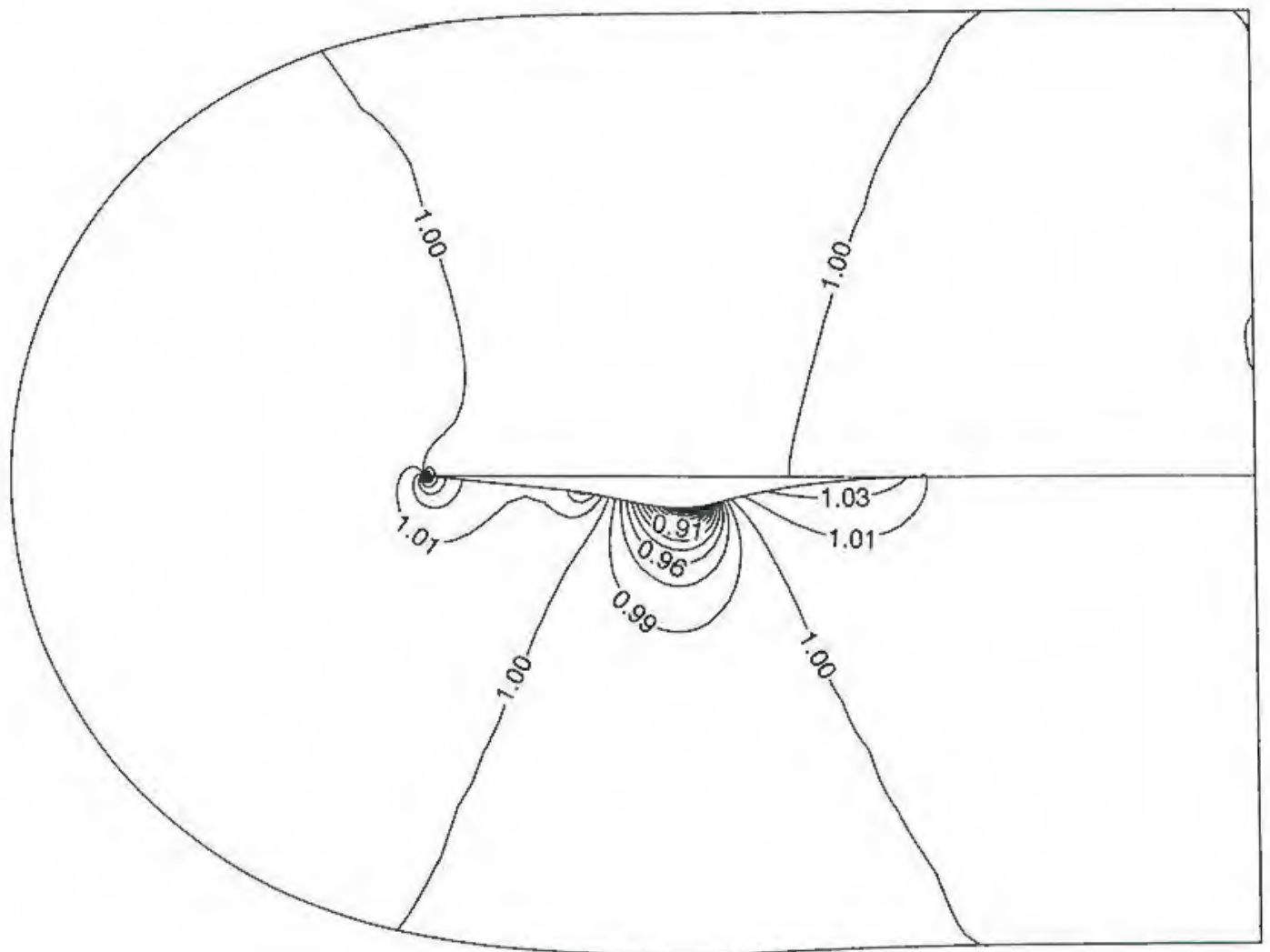

Figure 4.33: Side view of nondimensionalized pressure distribution over the vehicle $\left(M_{\infty}=0.65\right)$.

combination result of Eq. 4.40 (labeled "Sp", for supersonic flow only). For the upper surface, only the 2-D solutions will be compared to the computational results. Since the upper surface has an expansion, the axisymmetric geometry created by taking a longitudinal slice will have a negative cross-section. All theoretical results presented took an average of approximately 10 seconds of CPU time (per Mach number) on a Sun Ultra 10, compared to hours for each computational result.

A side view of the 3-D, inviscid, subsonic, computational solution on the full vehicle geometry is shown in Fig. 4.33. In general, the side view of the solution 

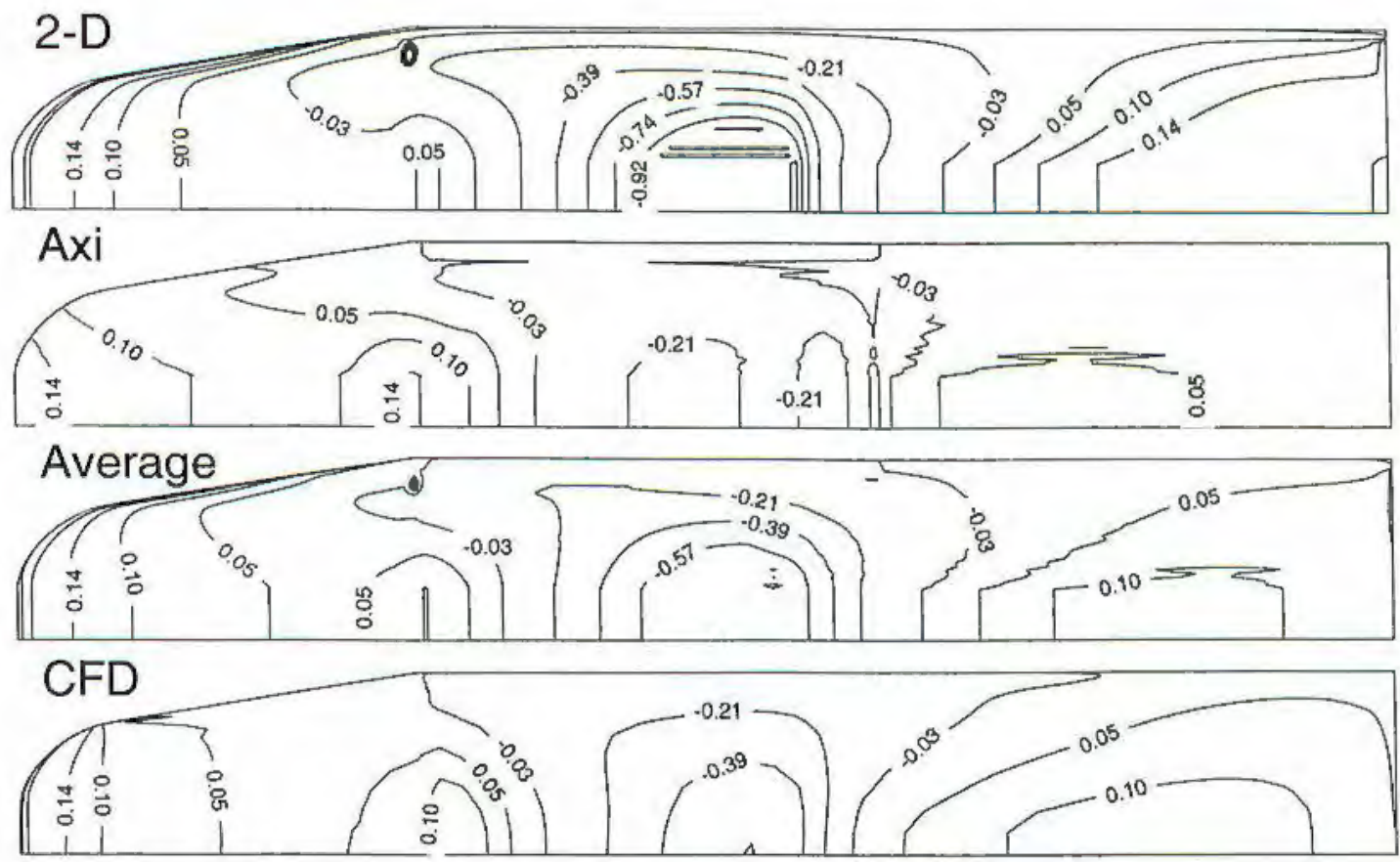

Figure 4.34: Pressure coefficient distribution on the lower surface of the vehicle $\left(M_{\infty}=0.65\right)$.

has a strong resemblance to the subsonic, half airfoil solution shown in Fig. 4.8. Pressure leakage from the lower to the upper surface occurs at the leading and trailing edges, causing a reduction in pressure on the lower surface.

Line contours of pressure coefficient on the bottom surface and normalized pressure on the top surface of the vehicle at Mach 0.65 are presented in Figs. 4.34 and 4.35 , respectively. The forces on the vehicle, as well as the percent error with respect to the CFD solution, are shown in Table 4.1 for the lower surface and Table 4.2 for the top surface. All forces are in units of $\mathrm{m}^{2}$ (the form of pressure used to calculate the forces was $p / p_{\infty}$ ). The drag force (labeled " $\mathrm{D}$ ") is the pressure force on the front side of the vehicle, the thrust force (labeled "T") is the pressure force on the rear side of the vehicle.

For all of the axisymmetric solutions to be presented, there are some os- 


\begin{tabular}{|c|c|c|c|c|c|c|}
\hline$M_{\infty}$ & & 2 -D & Axi & Avg & Sp & CFD \\
\hline 0.65 & $L$ & 717 & 741 & 729 & - & 737 \\
& $D$ & 34.5 & 36.3 & 35.4 & - & 35.9 \\
& $T$ & 36.0 & 37.5 & 36.7 & - & 37.3 \\
& $\% L$ & -2.7 & 0.5 & -1.1 & - & - \\
& $\% D$ & -3.9 & 1.2 & -1.4 & - & - \\
& $\% T$ & -3.5 & 0.4 & -1.6 & - & - \\
\hline 1 & $L$ & 673 & 755 & 714 & - & 721 \\
& $D$ & 45.5 & 39.2 & 42.3 & - & 37.3 \\
& $T$ & 20.6 & 35.6 & 28.1 & - & 32.5 \\
& $\% L$ & -6.7 & 4.7 & -1.0 & - & - \\
& $\% D$ & 21.8 & 5.1 & 13.5 & - & - \\
& $\% T$ & -37 & 9.4 & -14 & - & - \\
\hline \multirow{1}{*}{1.65} & $L$ & 761 & 759 & 760 & 763 & 707 \\
& $D$ & 51.6 & 42.1 & 46.8 & 50.7 & 42.8 \\
& $T$ & 24.0 & 32.5 & 28.3 & 25 & 25.1 \\
& $\% L$ & 7.7 & 7.4 & 7.5 & 7.9 & - \\
& $\% D$ & 20.5 & -1.6 & 9.4 & 18.5 & - \\
& $\% T$ & -4.2 & 29.6 & 12.7 & -0.4 & - \\
\hline
\end{tabular}

Table 4.1: Forces on lower surface of the vehicle $\left(\mathrm{m}^{2}\right)$. 


\begin{tabular}{|c|c|c|c|}
\hline$M_{\infty}$ & & $2-\mathrm{D}$ & $\mathrm{CFD}$ \\
\hline 0.65 & $L$ & -743 & -740 \\
& $D$ & 2.6 & 2.6 \\
& $T$ & 0.9 & 1.0 \\
& $\% L$ & 0.4 & - \\
& $\% D$ & -1.5 & - \\
\hline 1 & $\% T$ & -3.1 & - \\
\hline & $L$ & -737 & -727 \\
& $D$ & 2.4 & 2.5 \\
& $T$ & 0.9 & 0.9 \\
& $\% L$ & 1.4 & - \\
& $\% D$ & -7.5 & - \\
& $\% T$ & 1.1 & - \\
\hline 1.65 & $L$ & -748 & -744 \\
& $D$ & 2.7 & 2.6 \\
& $T$ & 0.9 & 1.0 \\
& $\% L$ & 0.6 & - \\
& $\% D$ & 4.7 & - \\
& $\% .2$ & - \\
\hline
\end{tabular}

Table 4.2: Forces on upper surface of the vehicle $\left(\mathrm{m}^{2}\right)$. 


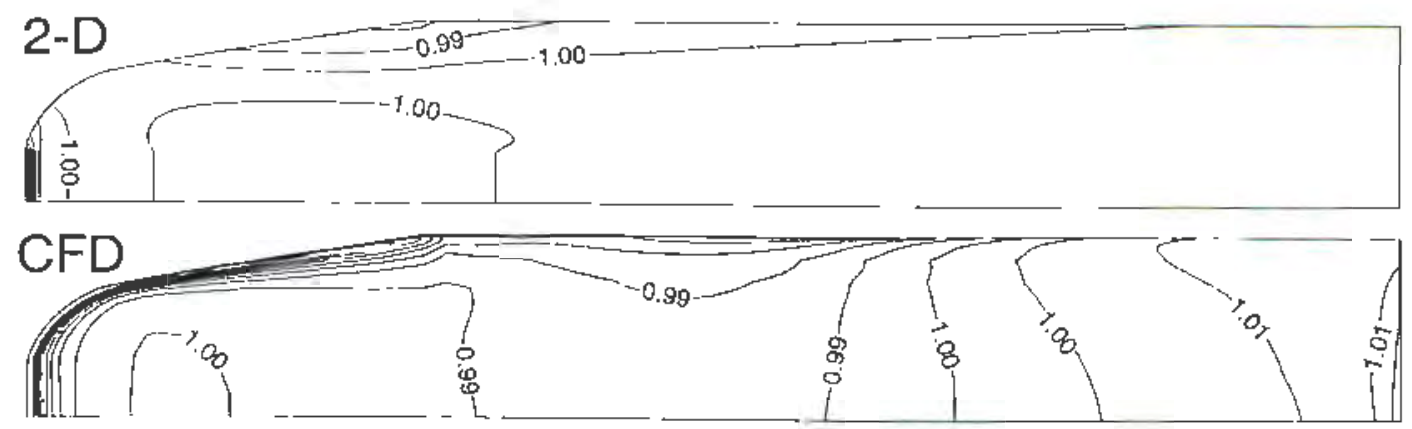

Figure 4.35: Normalized pressure distribution on the top surface of the vehiche $\left(M_{\infty}=0.65\right)$.

cillations in the pressure coofficient contour distribution (especially in the rear portion of the velicle). These numerical oscillations are a direct result of the seventh-order spline used to smooth the thind derivative in cross-sectional area. Integration and diffcrentiation of these geometries yields some terms in $x$ which are raised to as high as the twelfth power. Even ruming the numerical integration using double precision, oscillations arise when cortain integrals went to zero. This effect was mitigated in the present discussion by climinating these integrals when they tonded toward zero. An obvious fix to this problem would be to use a smoother geometry from the start, as was discussed earlier.

The subsonic results on the lower surface assuming 2-D fow, axisymmetric flow, and an average of the two are compared to the conputational solution for pressure coefficient in Fig. 4.34. In general, all three solutions model both the trends and magnitudes of the pressure on the vehicle, shown by comparing the force results in Table 4.1. The maximutn percent error for any of the methods is $-3.9 \%$ for the $2-\mathrm{D}$ drag force. The axisymmetric solution does the best jol of nnodeling the forces on the vehicle (maximam error of $1.2 \%$ ). The averaged solution does the best job of modeling the overall pressure trends on the vehicle, especially the rear portion of the vehicle, where both the magnitude and the 
distribution are seen to be in excellent agreement. Considering the discussion of Fig. 4.31, tho 2-D and axisymmetric solution along the centerlino bracket the computational solution over the majority of the profle. Thus, the average of the two solutions should be expected to give good predictions of pressure magnitude and distribution. Note that using Eq. 4.40 to combine the 2-D and axisymmetric solution resulted in an "optimal" value of 0 for the exponent $n$ (corresponding to purely axisymmetric fow).

The subsonic results on the upper surface assuming 2-D flow are compared to the computational solution for nondimensionalized pressure in Fig. 4.35. In general, the upper surface of the geometry has very little pressure variation (varying by $\pm 1 \%$ over the majority of the fuselage). The only significant variation in pressure occurs at the leading edge because of the flow expansion from the lower to the upper surface. The 2-D model captures the quantitative trends very well, but tends to be qualitatively less accurate near the trailing edge of the fuselage. The forces on the upper surface are compared to the CFD solution in Table 4.2. The maximum error for all forces calculated on the upper surface was $-3.1 \%$ for the thrust. However, observing that the thrust is approximately $0.1 \%$ of the lift on the upper surface, this error is not significant.

A side view of the 3-D, inviscid, sonic, computational solution on the full vehicle geometry is shown in Fig. 4.36. The lower surface is observed to have leading edge pressure leakage (as seen in the half airfoil solution in Fig. 4.15). The rearward portion of the lower surface generates a shock wave on the surface of the geometry similar to that found for the axisymmetric solition in Fig. 4.25. On the upper surface, a weak shock is observed near the trailing edge of the vehicle.

The sonic results on the lower surface assuming 2-D flow, axisymmetric flow, and an average of the two are compared to the computational solution for pressure coefficient in Fig, 4,37. Overall, the theory predicts the pressure distribution 


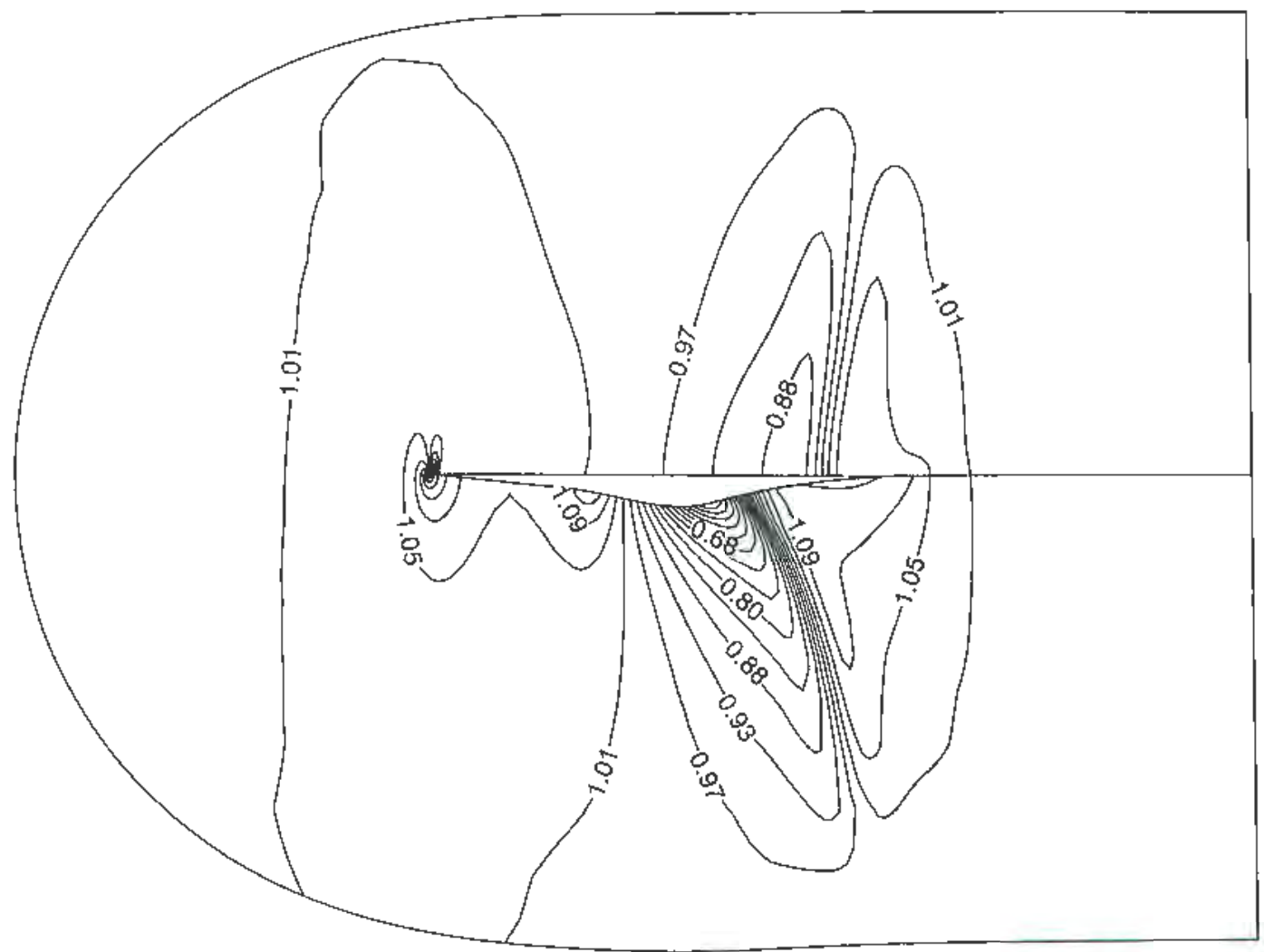

Figure 4.36: Side viow of nondimensionalized pressure distribution over the vehicle at sonic Mach number.

over the subsonic vehicle better than the sonic vehicle. The maximum percent crror in force prediction was found to be $-37 \%$ for the $2-\mathrm{D}$ solution. In general, the two dimensional solution was found to be a poor prediction of the vehicle flowfield, overpredicting the forobody region by over a factor of three and underpredicting the rear portion of the vehicle by about the sarne.

The best force prediction came from the axisymmetric solution, where the maximum percent error in force was $9.4 \%$ for the thrust. This is not a surprising result, since the area rule uses an equivalont axisymmetric goometry to find the drag force. Comparing the pressure profiles, the axisymmotric solution models 

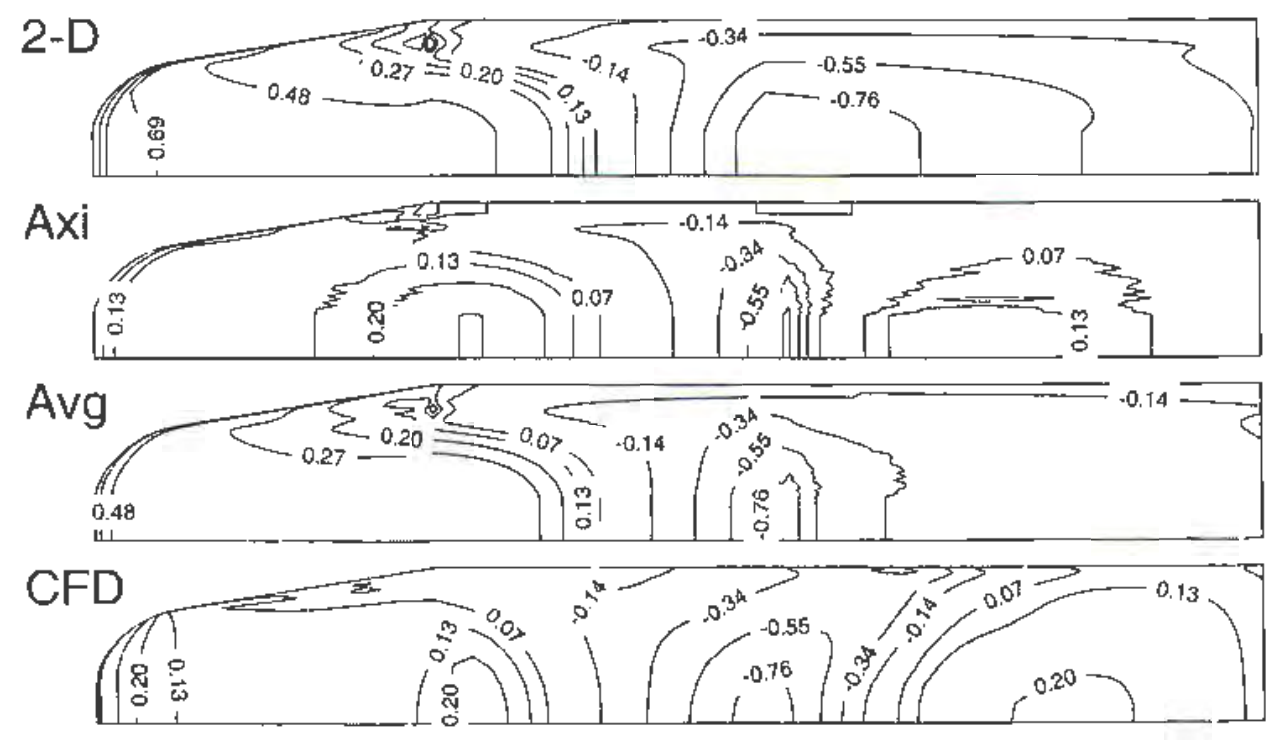

Figure 4.37: Sonic pressure coefficicnt distribution on full vehicle.

the forward portion of the geometry quite well (error in drag of 5.1\%), and tends to underpredict the rear portion of the vehicle (shown by the thrust calculation and Fig. 4.37). The averaged solution resulted in the lowest error in lift prediction of $-1.4 \%$, but was off for the drag by $13.5 \%$ and the thrust by $-14 \%$. This is a direct result of the overprediction in peak pressures for the 2-D solution. Note that using Eq. 4.40 to combine the 2-D and axisynnmetric solution resulted in an "optimal" value of 0 for the exponent $n$ (corresponding to purely axisymmetric llow).

The sonic results on the upper surface assuming 2-D flow are compared to the computational solution for nondimensionalized pressure in Fig. 4.38. The shock on the upper surface is clearly identifiable, with a compression ratio of about 1.2 . The analytical model is found to be a poor prodictor of the qualitative trends on the upper surface, but gives rough estimations of the quantitative forces on 


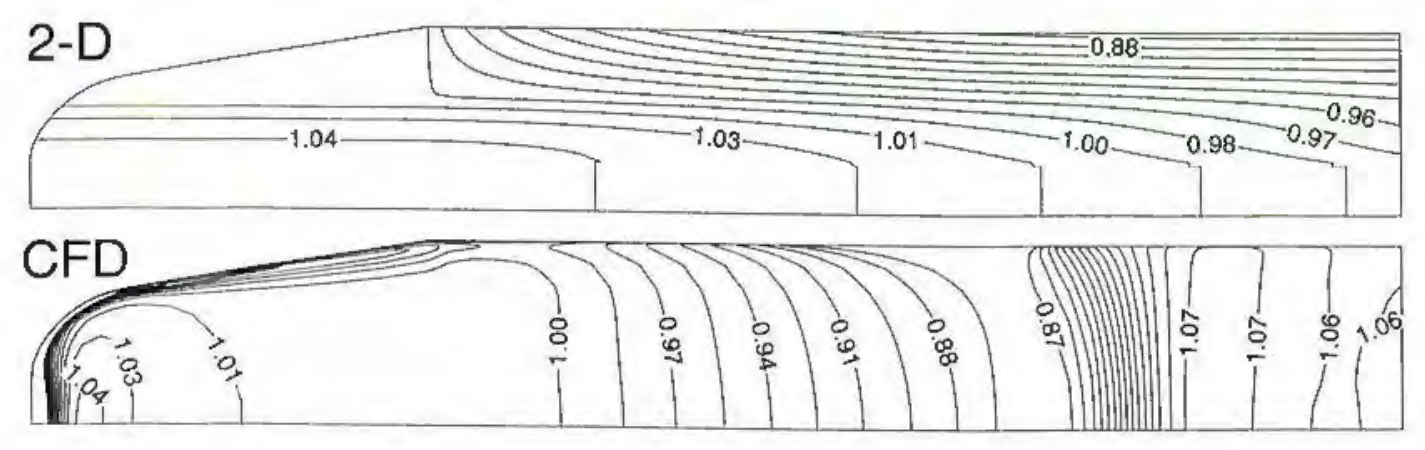

Figure 4.38: Normalized pressure distribution on the top surface of the vehicle at sonic Mach number.

the upper surface. The flowfield solution on the upper surface is affected by the pressure leakage occurring at the leading edge from the lower surface. Hence, the analytical model (which assumes no interaction from the lower surface) will not be able to predict the trends observed in Fig. 4.38. From Table 4.2, the maximum error in the forces calculated on the upper surface was $-7.5 \%$ for the drag force. However, considering that the drag force is $0.3 \%$ of the lift force, this error is not significant.

A side view of the 3-D, inviscid, supersonic, computational solution on the full vehicle geometry is shown in Fig. 4.39. The lower surface of the fuselage has an attached shock at the leading edge. The flow is rather nonuniform following the leading edge because of cross-flow along the fuselage. A second shock is at the end of the linear portion of the fuselage, followed by an expansion over the maximum thickness location. A third shock forms near the rear of the body in the compression region prior to the trailing edge of the fuselage, followed by a final weak shock at the trailing edge. The upper surface has little pressure variation, with only weak waves generated due to the slight amount of surface curvature.

The supersonic results on the lower surface assuming 2-D fow, axisymmetric 


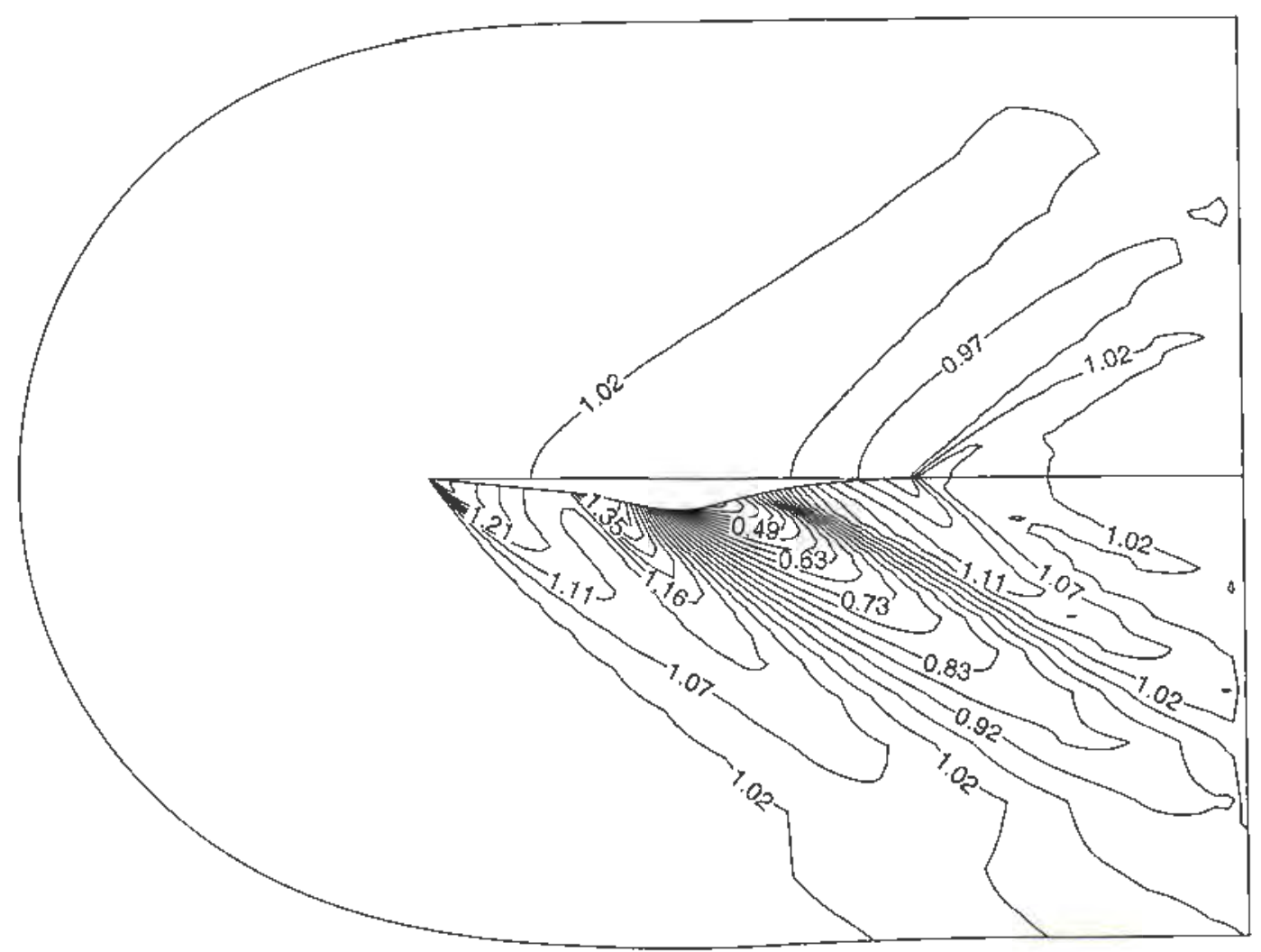

Figure 4.39: Side view of nondimensionalized pressure distribution over the vehicle $\left(M_{\infty}=1.65\right)$.

How, an averago of the two, and the combination described by Eq. $4.40(n=0.4)$ are compared to the computational solution for pressure cocihcient in Fig. 4.40. Overall, the theory does the worst job in predicting the supersonic flowficl. The maximum error found was $29.6 \%$ for the axisymmetric thrust calculation. The minimum crror found was $-0.4 \%$ for the thrust prediction using Eq. 4.40. All methods predicted lift to within $8 \%$, with the axisymmetric solution yielding the best result with $7.4 \%$. The 2-D solution again overpredicts the forebody region of the vehicle, with an crror in drag prediction of $20.5 \%$, howover the thrust. prediction was to within $-4.2 \%$. The axisymmetric solution predicted the drag 

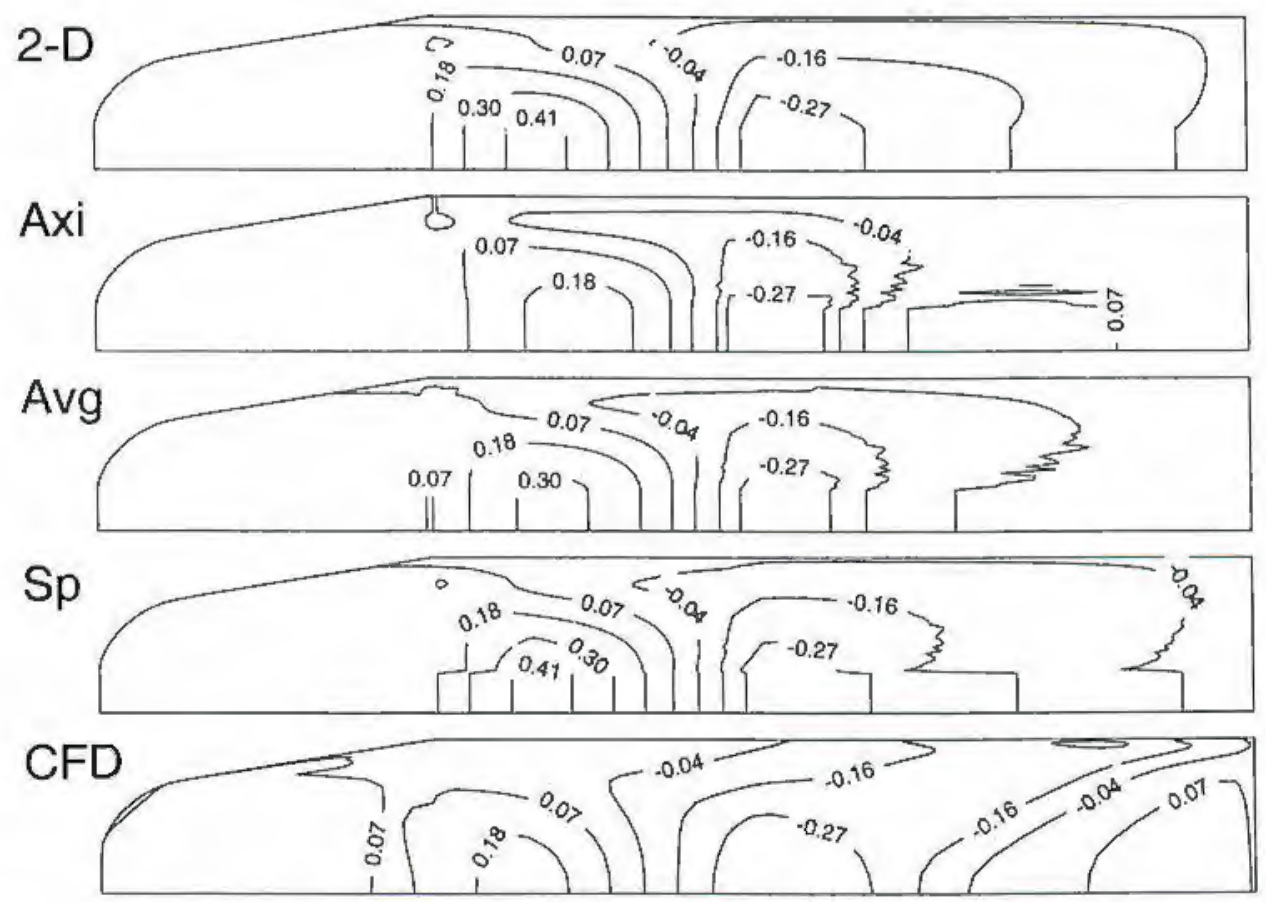

Figure 4.40: Pressure coefficient distribution on lower surface of vehicle $\left(M_{\infty}=\right.$ 1.65).

to within $-1.6 \%$, but overpredicted the thrust. The average solution yields a maximum error of $12.7 \%$ for the thrust calculation, and a minimum error of $7.5 \%$ for the lift calculation. It is suggested that the best means of predicting the forces on the supersonic vehicle is to use any of the lift predictions, the drag prediction from the axisymmetric solution and the thrust prediction from the 2-D or the combination solutions.

The supersonic results on the upper surface assuming 2-D flow are compared to the computational solution for nondimensionalized pressure in Fig. 4.41. As was observed in Fig. 4.39, there is little pressure variation on the upper surface. Except near the leading and trailing edge, the computational result predicts a pressure variation of around $\pm 5 \%$. This agrees well with the analytical result, which also predicts pressure variations of around $\pm 5 \%$. Two features on the 


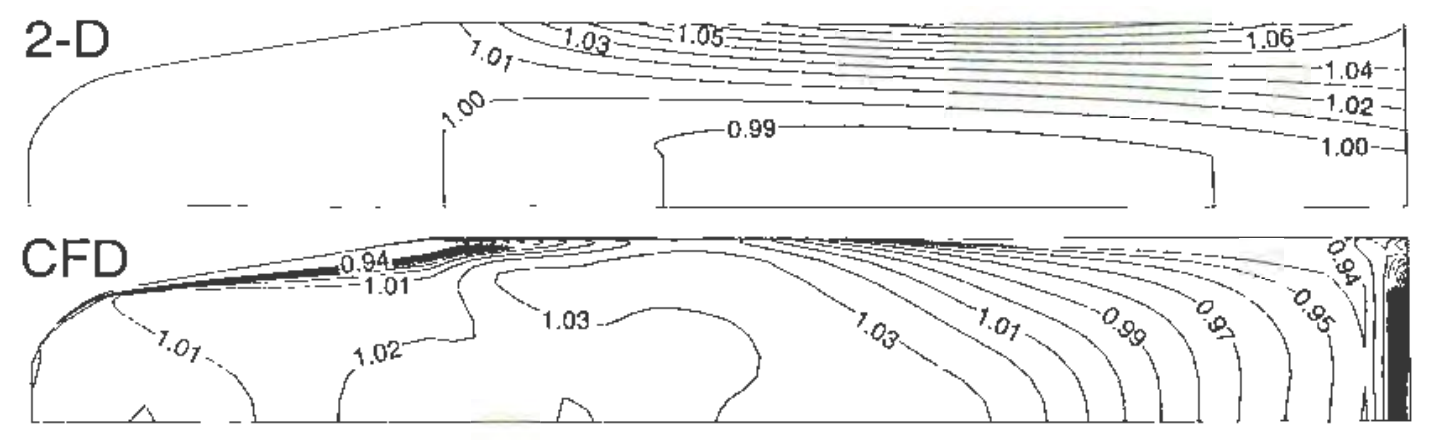

Figure 4.41: Pressure coefficient distribution on upper surface of vehicle $\left(M_{\infty}=\right.$ $1.65)$.

computational solution are not predicted with the analytical model: the expansion occurring on the leading edge of the forebody and the shock occurring at the trailing edge of the vehicle. The expansion is not predicted due to the assumption of zero cross-flow. The trailing edge shock is due to the flow exiting the lower surface, thus the model will not predict this trend because it assumes no flow communication between the lower and upper surface. From Table 4.2, the maximum error in force prediction was $-5.2 \%$ for the thrust calculation. Howover, the magnitude of the thrust is very sinall ( $\sim 0.1 \%$ of the lift) so that the crror is determined to not be significant.

The total lift and drag on the velicle using the two-dimensional and axisymmetric modeling (where the upper surface is solved using the 2-D method) is compared to the computational solutions in Table 4.3. For the sulssonic results, the error in net lift prediction was $945 \%$ for the $2-\mathrm{D}$ modeling and $-36.7 \%$ for the axisymmetric modeling. These errors are indeed quite high. However, upon inspection of the net lift predicted by the computational solutions, the lift on this geometry is very small (approximatcly $0.3 \%$ of the average lift force on the lower and upper surface). The analytical predictions for lift were found to be $3.5 \%$ for the $2-\mathrm{D}$ modoling and $0.2 \%$ for the axisymmetric modeling. Thus, although the 


\begin{tabular}{|c|c|c|c|c|}
\hline$M_{\infty}$ & & $2-\mathrm{D}$ & Axi & CFD \\
\hline 0.65 & $L_{\text {tot }}$ & -25.9 & -1.6 & -2.5 \\
& $D_{\text {tot }}$ & 0.1 & 0.5 & 0.2 \\
& $\% L_{\text {tot }}$ & 945 & -36.7 & - \\
& $\% D_{\text {tot }}$ & -52.2 & 126 & - \\
\hline 1 & $L_{\text {tot }}$ & -64.6 & 17.4 & -6.2 \\
& $D_{\text {tot }}$ & 26.3 & 5.0 & 6.4 \\
& $\% L_{\text {tot }}$ & 935 & -379 & - \\
& $\% D_{\text {tot }}$ & 311 & -21 & - \\
\hline 1.65 & $L_{\text {tot }}$ & 13.2 & 11.3 & -36.9 \\
& $D_{\text {tot }}$ & 29.3 & 11.4 & 19.3 \\
& $\% L_{\text {tot }}$ & -136 & -131 & - \\
& $\% D_{\text {tot }}$ & 51.7 & -41.1 & - \\
\hline
\end{tabular}

Table 4.3: Total forces on the vehicle $\left(\mathrm{m}^{2}\right)$.

analytical methods do not quantitatively predict the correct value of lift, they do qualitatively predict that there will be very little lift on this geometry. This argumont holds true for the rest of the lift force errors reported in Table 4.3. However, the analytical methods are found to better predict the net drag on the vehicle, but still these crrors are a bit high. In Chapter 7 , a method for roducing the lift and drag errors will be proposed that captures the overall behavior of the geometry for the full range of Mach numbers. 


\section{Chapter 5}

\section{Hypersonic Off-Design Aerodynamic}

\section{Performance}

The development of the transonic off-design methodology in Chapter 4 is only applicable up to Mach numbers in the low supersonic range. In this chapter, the methods used to predict the hypersonic off-design aerodynamics on the vohicle are discussed. The methods are applied to a full vehicle geometry and validated using 3-D, inviscid computational solutions.

\subsection{Forebody}

The off-design aerodynamic performance for the waverider forebody is calculated using a quadratic interpolation between tangent-wedge and tangent-cone theory reported by Grantzis:

$$
C_{p_{\mathrm{q} \text { thid }}}=\left(1-\left(\frac{\beta-\theta}{\beta-\theta_{c}}\right)^{2}\right) C_{p_{\mathrm{tw}}}+\left(\frac{\beta-\theta}{\beta-\theta_{c}}\right)^{2} C_{p \mathrm{tc}}
$$

where $C_{p_{\text {quad }}}$ is the pressure coefficient predicted by the quadratic interpolation at the point of solution, $\beta$ is the shock angle created by the deflection of the surface (including angle of attack), $\theta$ is the angle between the leading edge of the 
generating cone and the point of solution on the waverider, $\theta_{c}$ is the angle of the generating cone, $C_{p_{t w}}$ is the pressure coefficient predicted using tangent-wedge theory and $C_{p_{t c}}$ is the pressure coefficient predicted using tangent-cone theory. Grantz's method is slightly modified for this study by using the exact solution for tangent-wedge and tangent-cone instead of the approximate relations given by Grantz. The Mach number on the surface is calculated in a similar manner as the pressure coefficient

$$
M_{\text {quad }}=\left(1-\left(\frac{\beta-\theta}{\beta-\theta_{c}}\right)^{2}\right) M_{t w}+\left(\frac{\beta-\theta}{\beta-\theta_{c}}\right)^{2} M_{\text {tc }}
$$

The temperature on the surface is then calculated using isentropic compression from the conditions behind the conical shock to the waverider surface. The upper surface of the forebody is assumed to be wedge flow for negative angles of attack and Prandtl-Meyer flow for positive angles of attack.

Solutions based on Grantz's method were numerically validated for both angle of attack and Mach number by Takashima ${ }^{5}$. The forebody planform shape for Takashima is plotted in Fig. 5.1 and the forebody cross-section shape for Takashima is plotted in Fig. 5.2. Overlaying Takashima's geometry in both figures is the current model's geometry. Plotted in Fig. 5.2 are contours of pressure from Takashima's GASP solution at Mach 10 and zero angle of attack as well as the prescribed shock shape for the current model. Figures 5.1 and 5.2 show that there are subtle differences between the two geometries. The shock shape prescribed for the current model geometry fits well with the GASP solution for shock shape (the shock shape used in Takashima's geometry is unknown). The geometry differences and unknown shock shape account for the small errors in lift and drag calculations between the current model and Takashima's model to be presented below.

The inviscid L/D performance of the forebody at Mach 10 is plotted as a function of angle of attack in Fig. 5.3 for the current model, Takashima's geom- 


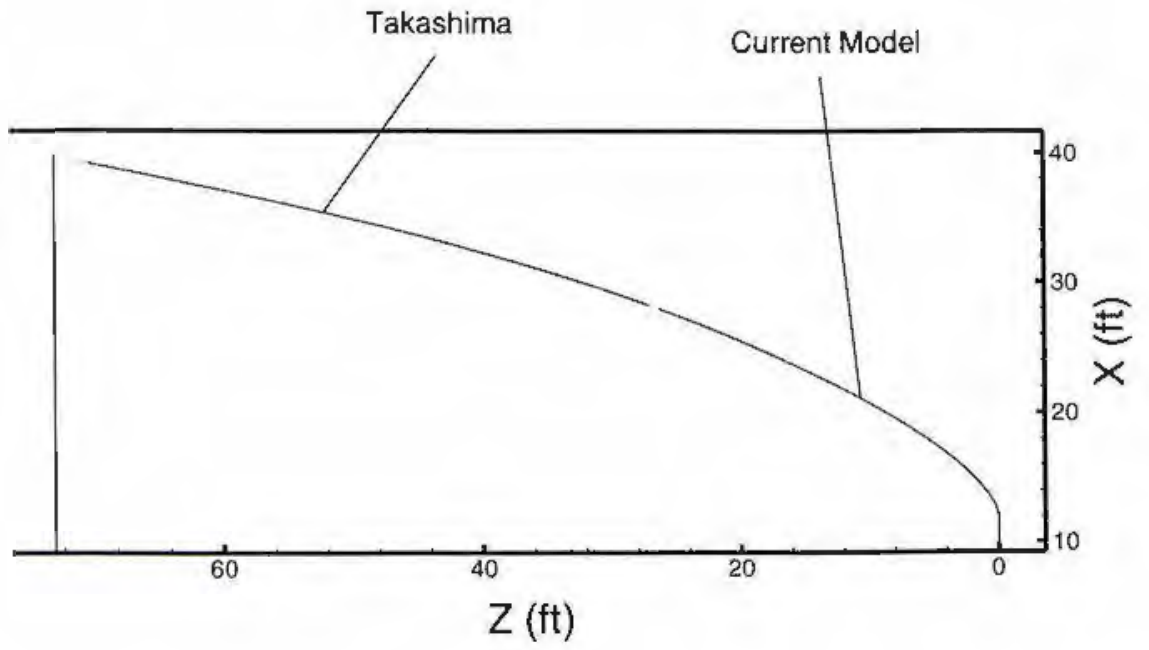

Figure 5.1: Current geometry overlayed by Takashima's geometry, planform $\left(M_{\infty}\right.$ $=10, \alpha=0)$.

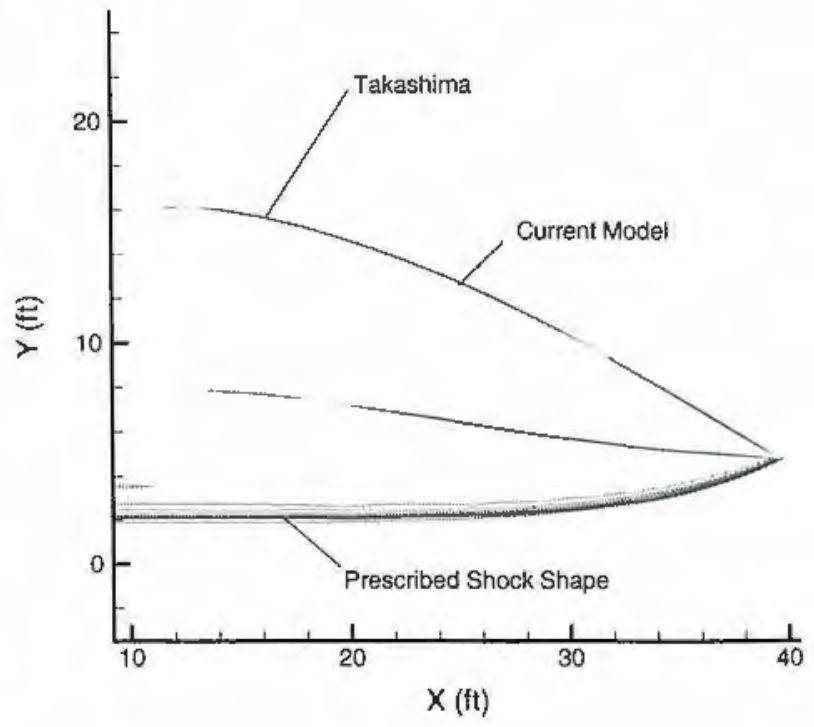

Figure 5.2: Current geometry overlayed by Takashima's geometry, including GASP solution for pressure contours, cross-section $\left(M_{\infty}=10, \alpha=0\right)$. 


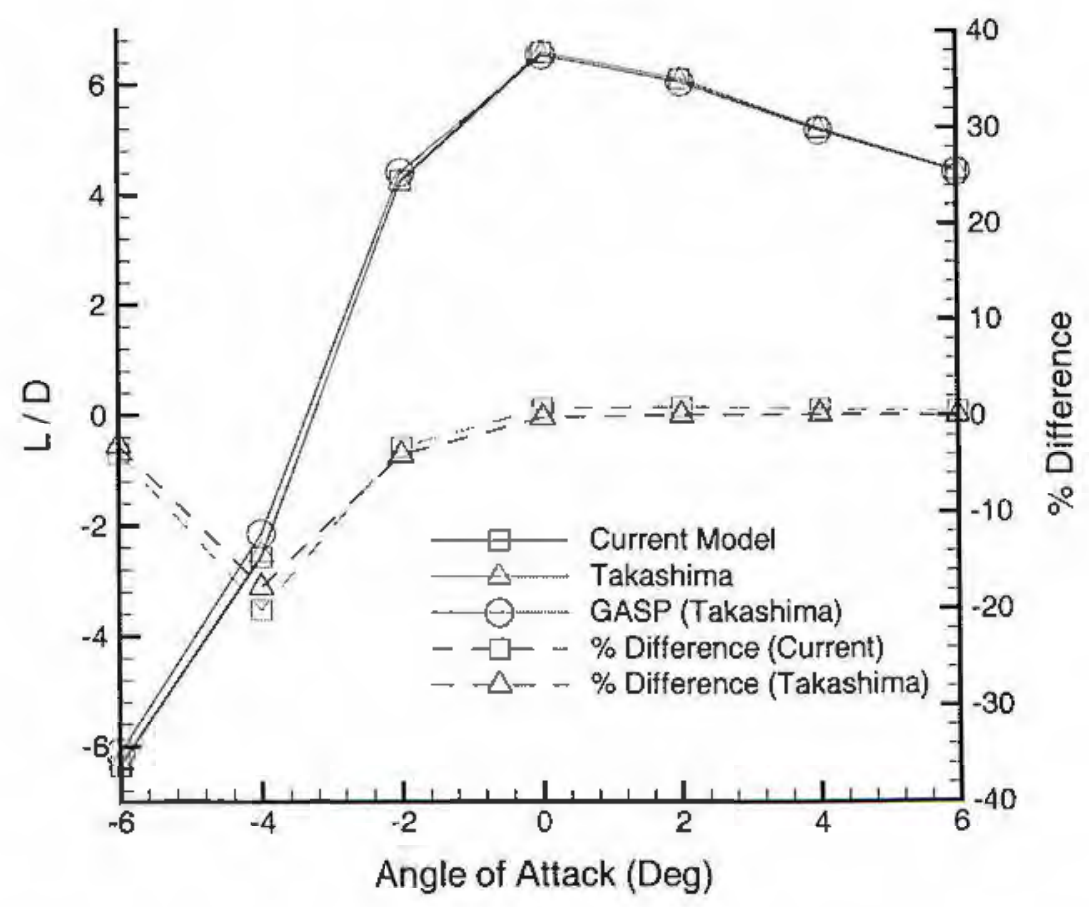

Figure 5.3: Inviscid L/D as a function of angle of attack $\left(M_{\infty}=10\right)$.

etry and the GASP result. The current model is in agreement with Takashima's results. The maximum \% difference between the analytical models and the computational results is approximately $20 \%$. The angle of attack representing this error is close to the zero lift angle of attack, thus the error seen in $\mathrm{L} / \mathrm{D}$ is due to the small magnitude of lift. Neglecting the zero lift result, the maximum error in $\mathrm{L} / \mathrm{D}$ is under $4 \%$ for all cases.

A general trend shown by Fig. 5.3 is a steep reduction of L/D for negative angle of attack and a more gradual reduction of $\mathrm{L} / \mathrm{D}$ for positive angle of attack. This trend is frequently observed in hypersonic flight, and will be demonstrated several more times in Chapter 7. For negative angles of attack, shock compression on the upper surface tends to dramatically increase the negative lift on the geometry while also increasing wave drag. On the lower surface, negative angles 
of attack reduce the drag, but tend to substantially roduce the lift. The not effect is a clastic reduction in overall lift, with a modest increase in wave drag, resulting in the steep negative slope for $\mathrm{L} / \mathrm{D}$ found in Fig. 5.3. For positive angle of attack, shock compression is increased on the lower surface, resulting in highor lift and wave drag. However, the upper surface has an expansion for positive angle of attack, thus reducing negative lift. Also, with a positive angle of attack, the wave drag is reduced on the upper surface. If the upper surface is flat enough, a positive angle of attack will result in thrust on the upper surface, thus decreasing the overall drag. Combining the results of the lower and upper surface, the L/D has a more gradual decrease with positive angle of attack bocause both lift and drag are seen to incroase.

The inviscid $\mathrm{L} / \mathrm{D}$ performance of the forebody at zero angle of attack is plotted as a function of Mach number in Fig. 5.4 for the current model, Takashima's geometry and the GASP result. The current model is off by about $1 \%$ for all casos in comparison to Takashima and the GASP results. This offset is quite small and is a result of the small errors in geomotry and shock shape between the current model and Takashima's geometry.

Figure 5.4 also has a general trend that is commonly observed for lypersonic flow. The L/D of the waverider geometry tends to decrease with a decrease in Macl number. There are two reasons for this decrease in performance: lower shock compression due to lower Mach number and pressure leakage due to the shock wave not being attached to the leading edge. Considering the oblique shock cquations, for a constant wedge angle, decreasing freestream Mach number tends to decrease the compression ratio across the shock, resulting in less lift and drag. However, since those goometries have a relatively shallow compression angle, a reduction in pressure will have more of an effect on the lift than the drag. Thus, the L/D will decrease with Mach number. Pressure leakage over the leading edge has less of an impact on waverider performance (which has beem demonstrated 


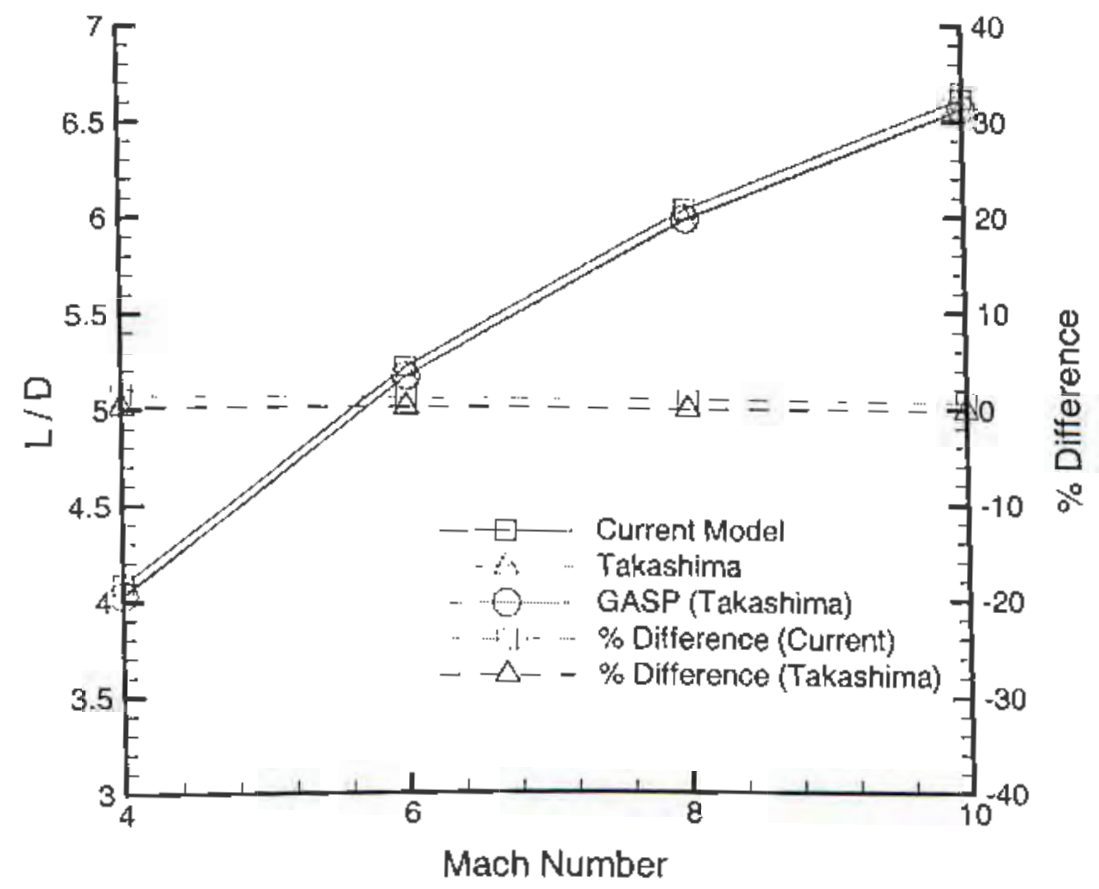

F'igure 5.4: Inviscid L/D as a function of Mach number $(\alpha=0)$.

by Takashima, Cockrell ${ }^{28}$, and others). Leakage over the leading edges tends to locally lower surface pressure, but not nearly to the extent that shock strength reduction affects the performance.

\subsection{Fuselage}

The pressure distribution on the fuselage of the geometry is solved using shock-expansion theory, based on the flow exiting the waverider forcbody. Flow spillage over the sicle edge of the fuselage (from a detached shock wave) is incorporated into the nodel due to the high amount of compression occurring on the lower surface relative to the upper surface. The side edge pressure is assumed to be very low (essentially zero) duc to a large expansion. At the intersection 
of the waverider and the fuselage, a region of flow expansion occurs near the side edge. This is an artifact of the vehicle geometry definition. To incorporate leakage effects, a linear interpolation of the pressure is taken in the spanwise direction from the point at the trailing edge of the waverider where expansion begins to occur on the fuselage, to the side edge of the fuselage. This pressure distribution is then used as the flow exiting the waverider and is placed through the shock-expansion theory to calculate the pressure distribution on the rest of the vehicle.

\subsection{Validation}

Validation of the aerodynamics on the vehicle airframe for both Mach number and angle of attack is accomplished using CFD. OVERFLOW ${ }^{74}$ is again used to solve the inviscid, 3-D flowfield over the vehicle fuselage. The computational grid used is identical to the hyperbolic grid shown in Fig. 4.28, with the exception that the outer box grid and the wake region behind the vehicle are eliminated because of the high Mach numbers solved. Grid simplification was used to decrease the computational time necessary for solution. The vehicle geometry solved is identical to the Mach 12 geometry used to validate the transonic solutions.

\subsubsection{On-Design Conditions}

An isometric and side view of the computational solution for normalized pressure distribution over the Mach 12 vehicle at on-design conditions ( $\mathrm{M}=12$, $\alpha=0^{\circ}$ ) is shown in Fig. 5.5. The CFD results show that the leading edge shock is attached along the waverider forebody and lies close to the fuselage. At the exit plane solution, the shock wave is detached from the fuselage. However, the 


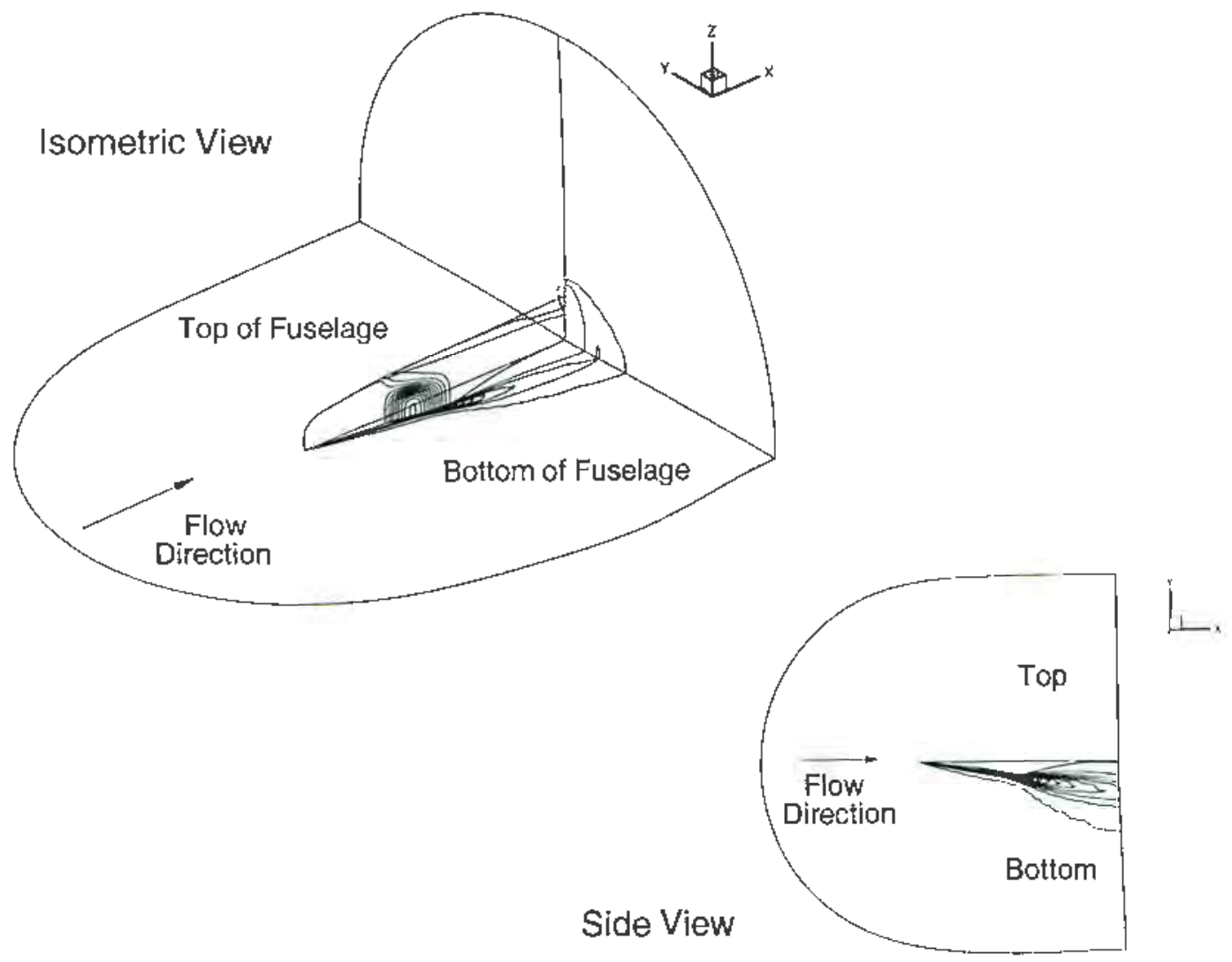

Figure 5.5: Normalized pressure distribution over Mach 12 veliclo fusclage at on-design conditions. 


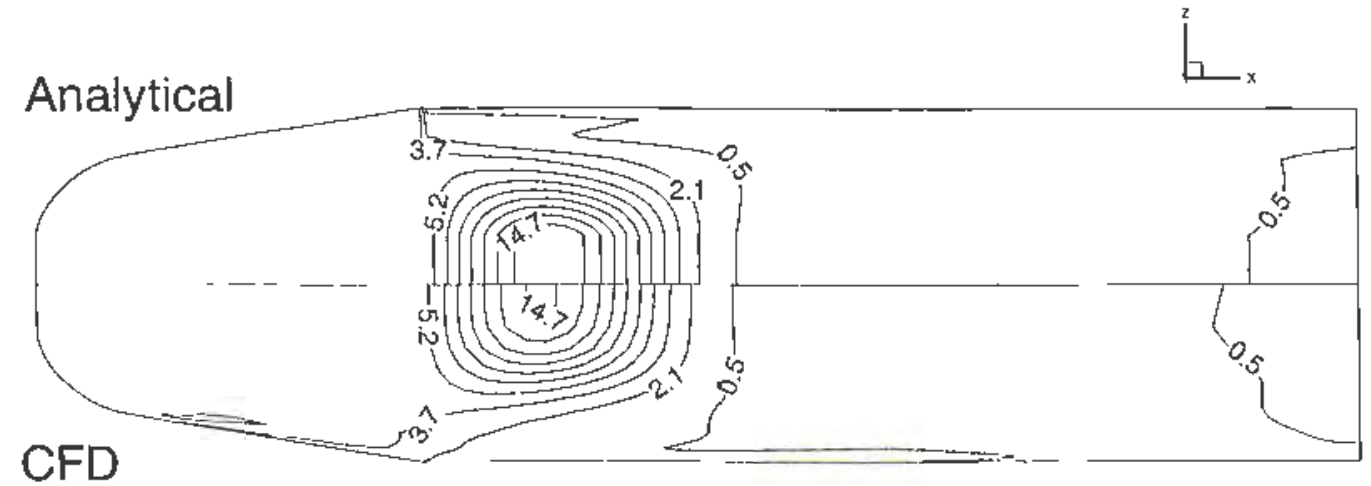

Figure 5.6: Normalized pressure distribution over lower surface of Mach 12 velicle fuselage al on-design conditions.

shock standoff distance is quite small and the shock is rather weak due to the expansion region on the rear of the fuselage. 'Thus, pressure leakage does not play a significant role in performance degradation.

The nornalized pressure distribution on the lower surface of the Mach 12 vehicle at on-design conditions is shown in Fig. 5.6. The forces calculated on both the lower and upper surface may be found in Table 5.1. In gencral, the analytical methods described above and in Chapter 2 givo cxcellent prodictions of both the quantitative and qualitative aspects of the flowhich. The pressure distribution is matched very well, with the only exception being an overprediction of peak pressure for the analytical model. This overprediction in pressure results in an overprediction of lift and drag on the lower surface of the fuselage of $5 \%$ and $9.5 \%$, respectively.

The normalized pressure distribution on the upper surface of the Mach 12 vehicle at on-design conditions is shown in Fig. 5.7. The forces on the upper surface are listed in Table 5.1. In general, the analytical inethods givo accurate predictions over the entire upper surface, with an overprediction in pressure near the fuselage side edges. The overprodiction is due to flow spillage that is not 


\begin{tabular}{|c|c|c|}
\hline & Analytical & CFD \\
\hline$L_{\text {bot }}$ & 1876 & 1787 \\
\hline$D_{\text {bot }}$ & 240 & 219 \\
\hline$T_{\text {bot }}$ & 4.4 & 7.4 \\
\hline$\% L_{\text {bot }}$ & 5.0 & - \\
\hline$\% D_{\text {bot }}$ & 9.5 & - \\
\hline$\% T_{\text {bot }}$ & -40 & - \\
\hline$L_{\text {top }}$ & -776 & -766 \\
\hline$D_{\text {top }}$ & 3.3 & 3.1 \\
\hline$T_{\text {top }}$ & 0.9 & 0.9 \\
\hline$\% L_{\text {top }}$ & 1.2 & - \\
\hline$\% D_{\text {top }}$ & 6.6 & - \\
\hline$\% T_{\text {top }}$ & -4.4 & - \\
\hline$L_{\mathrm{tot}}$ & 1100 & 1021 \\
\hline$D_{\text {tot }}$ & 238 & 214 \\
\hline$L / D$ & 4.6 & 4.8 \\
\hline$\% L_{\text {tot }}$ & 7.8 & - \\
\hline$\% D_{\text {tot }}$ & 11.3 & - \\
\hline$\% L / D$ & -3.1 & - \\
\hline
\end{tabular}

Table 5.1: On-design forces for vehicic fuselage $\left(\mathrm{n}^{2}\right)$. 


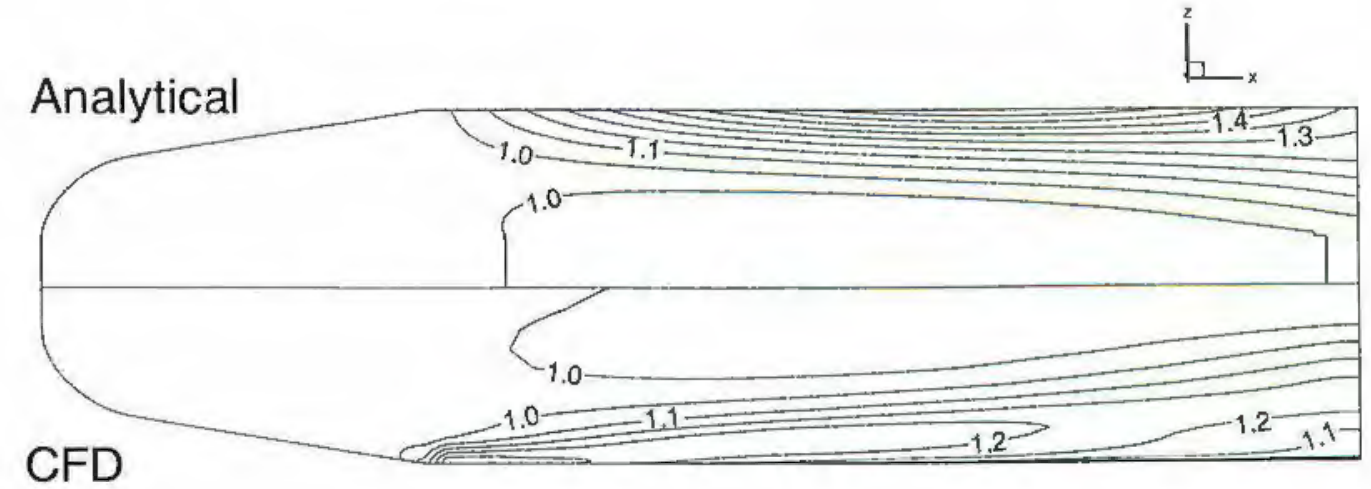

Figure 5.7: Normalized pressure distribution over upper surface of Mach 12 vehicle fuselage at on-design conditions.

modeled on the upper surface. The forces on the upper surface were found to be predicted very well, with the maximum error found to be $6.6 \%$ for the drag prediction.

Table 5.1 shows the prediction for net forces on the Mach 12 vehicle at ondesign conditions. The overprediction of peak pressure on the lower surface results in an overall overprediction of lift of $7.8 \%$ and an overprediction of drag of $11.3 \%$. The L/D, however, was underpredicted by only $3.1 \%$. Hence, the analytical model gives good predictions of the vehicle fuselage performance at the on-design conditions.

\subsubsection{Off-Design Conditions}

An isometric and side view of the computational solution for normalized pressure distribution over the Mach 12 vehicle at off-design conditions of $M=9$ and $\alpha=3^{\circ}$ are shown in Fig. 5.8. An expansion occurs over the upper surface of the fuselage because of the positive angle of attack incurred on the vehicle. Over the lower surface, the shock compression is decreased with respect to the 


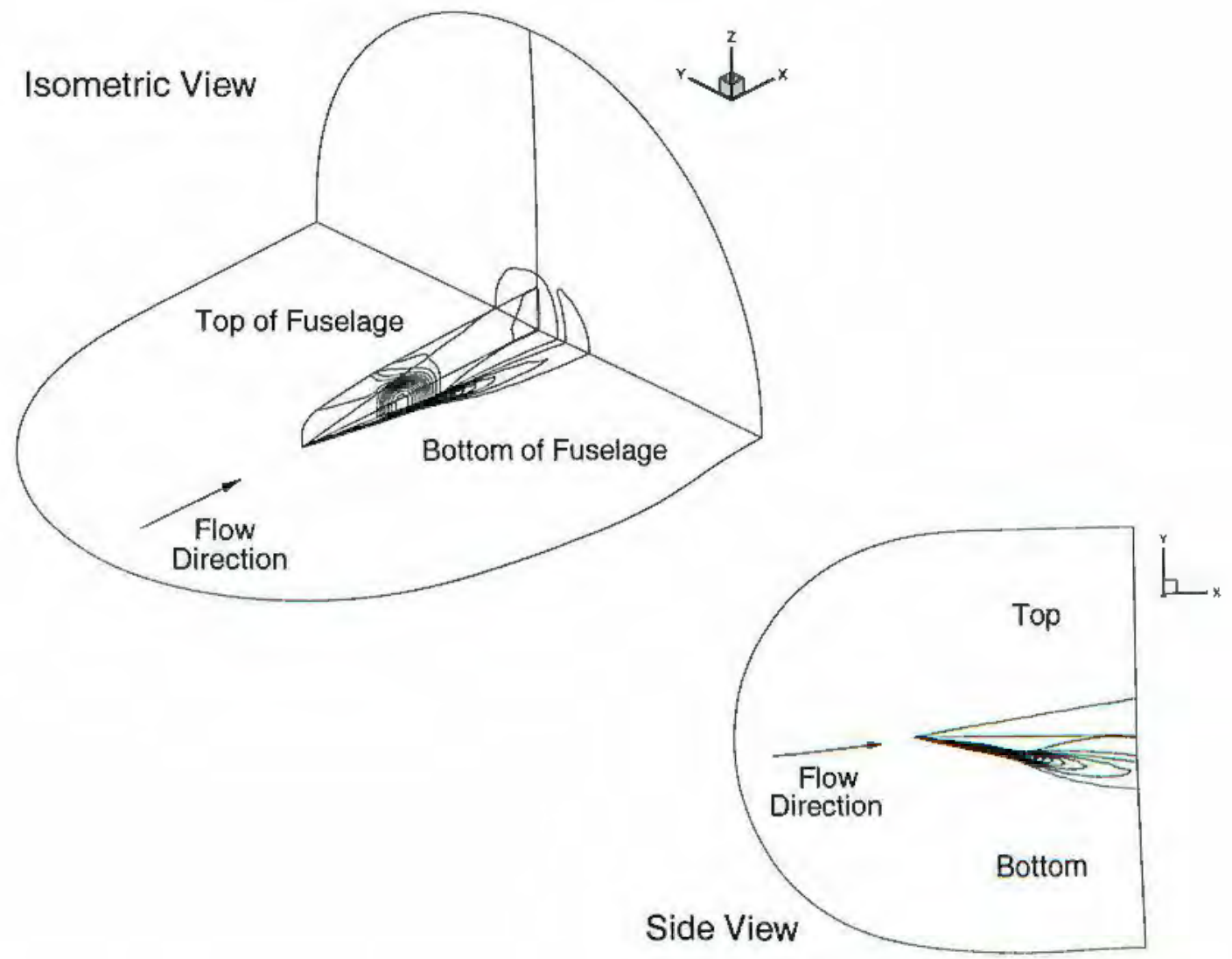

Figure 5.8: Normalized pressure distribution over Mach 12 vehicle fuselage $\left(M_{\infty}\right.$ $\left.=9, \alpha=3^{\circ}\right)$. 


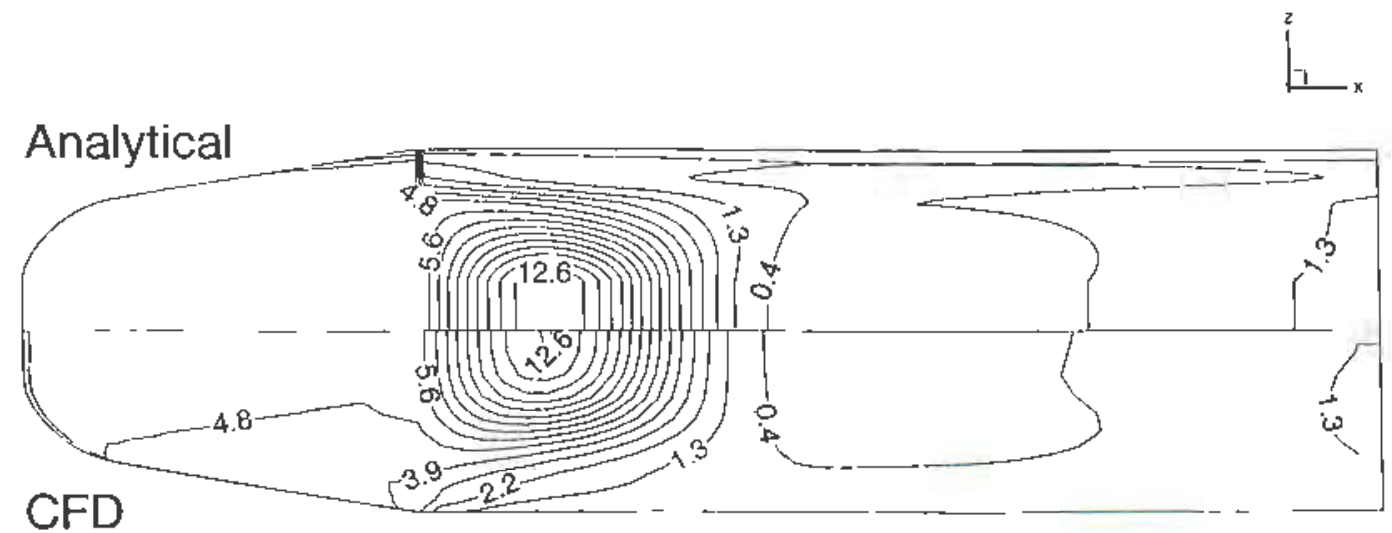

Figure 5.9: Normalized pressure distribution over lower surface of Mach 12 vehicle fisclage $\left(M_{\infty}=9, \alpha=3^{\circ}\right)$.

on-design Mach nunber but is increased with respect to the on-design angle of attack. Observing the base plane at the end of the vehicle fuselage, the shock wave hos close to the fuselage side edge, thus pressure leakage (although still present) is not large.

The normalized pressure distribution on the lower surface of the Mach 12 vehicle at off-design conditions of $\mathrm{M}=9$ and $\alpha=3^{\circ}$ is shown in Fig. 5.9. The forces calculated on both the lower and upper surface are found on the left side of Thable 5.2. "Two trends are observed that are typical of the amalytical method at off-clesign: overprediction of peak pressure on the fuselage and overprediction of pressure near the leading edge of the waverider forebody. The peak pressure in Fig. 5.9 was overpredicted by approximately $6 \%$ between the analytical and computational results. The pressure near the leading edge of the waverider is overpredicted by approximately $20 \%$, Both of these errors are due to the assumption of no cross-flow on the geonetry. At the peak prossure region and the leading edge region, spanwise pressure gradients incur velocitics in the crossflow direction, which are not accounted for in the current model. Hence, the 


\begin{tabular}{|c|c|c|c|c|}
\hline & \multicolumn{2}{|c|}{$\alpha=3^{\circ}$} & \multicolumn{2}{c|}{$\alpha=-3^{\circ}$} \\
\cline { 2 - 5 } & Analytical & $\mathrm{CFD}$ & Analytical & $\mathrm{CFD}$ \\
\hline$L_{\text {bot }}$ & 2124 & 2006 & 783 & 772 \\
$D_{\text {bot }}$ & 346 & 316 & 57 & 53 \\
$T_{\text {bot }}$ & 5 & 6 & 7 & 10 \\
$\% L_{\text {bot }}$ & 5.9 & - & 1.4 & - \\
$\% D_{\text {lot }}$ & 9.3 & - & 7 & - \\
$\% T_{\text {bot }}$ & -9.9 & - & -32 & - \\
\hline$L_{\text {top }}$ & -382 & -398 & -1307 & -1326 \\
$D_{\text {tor }}$ & 0 & 0 & 71 & 73 \\
$T_{\text {top }}$ & 19 & 20 & 0 & 0 \\
$\% L_{\text {top }}$ & -4 & - & -1.5 & - \\
$\% D_{\text {top }}$ & 0 & - & -2.9 & - \\
$\% T_{\text {top }}$ & -5.6 & - & 0 & - \\
\hline$L_{\text {tol }}$ & 1742 & 1608 & -523 & -554 \\
$D_{\text {tot }}$ & 321 & 290 & 121 & 116 \\
$L / D$ & 5.4 & 5.5 & -4.3 & -4.8 \\
$\% L_{\text {tot }}$ & 8.3 & - & -5.5 & - \\
\hline$D_{\text {tot }}$ & 10.7 & - & 4.1 & - \\
\hline & -2.2 & - & -9.2 & - \\
\hline
\end{tabular}

Table 5.2: Forces on Mach 12 vehicle design at $\mathrm{M}=9\left(\mathrm{~m}^{2}\right)$. 


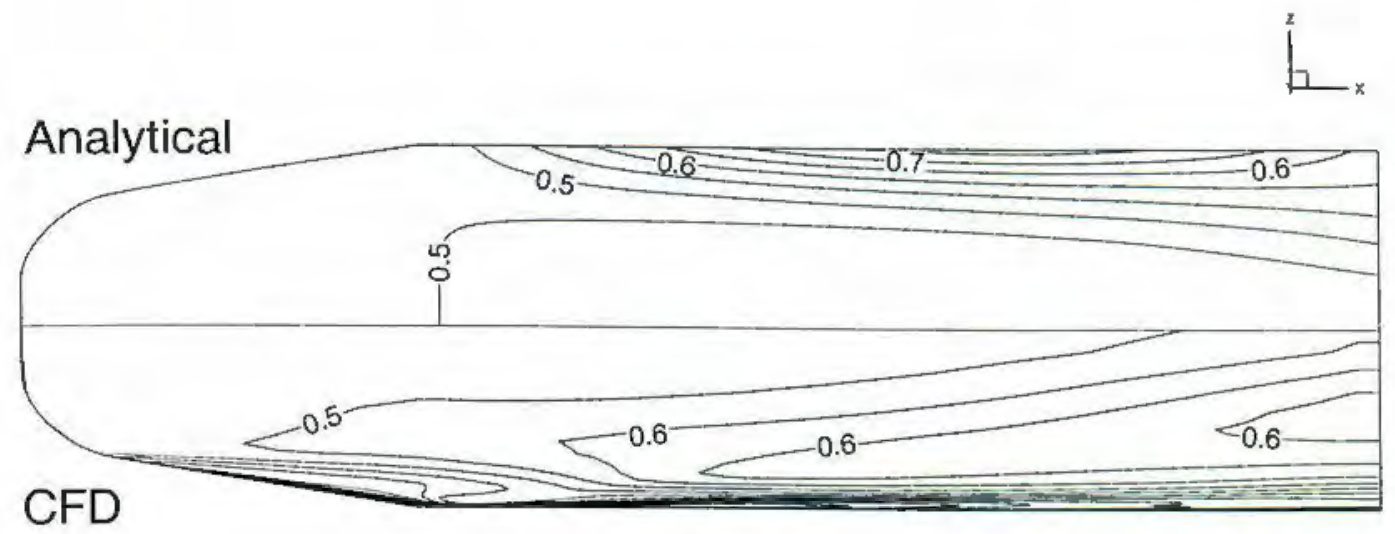

Figure 5.10: Normalized pressure distribution over upper surface of Mach 12 vehicle fuselage $\left(M_{\infty}=9, \alpha=3^{\circ}\right)$.

analytical model will tend to overpredict pressure forces on the vehicle, having errors of $5.9 \%$ and $9.3 \%$, respectively compared to CFD. The maximum error was in the thrust prediction, but the magnitude of thrust was very small compared to the drag and lift of the vehicle. Thus, this error is considered negligible.

The normalized pressure distribution on the upper surface of the Mach 12 vehicle at off-design conditions of $\mathrm{M}=9$ and $\alpha=3^{\circ}$ is shown in Fig. 5.10. The forces calculated on the upper surface are found on the left side of Table 5.2. The most apparent aspect of Fig. 5.10 is the effect of cross-flow on the upper surface for the computational results. Since the analytical methods developed herein do not consider cross-flow, the models have no way of matching the qualitative properties of the upper surface flowfield. However, the models do capture the quantitative properties quite well, resulting in errors of lift and thrust on the upper surface of $-4 \%$ and $-5.6 \%$, respectively.

The net forces on the Mach 12 vehicle at $\mathrm{M}=9$ and $\alpha=3^{\circ}$ are shown in Table 5.2. The net lift and drag on the fuselage are overpredicted by $8.3 \%$ and $10.7 \%$, respectively. This result is a direct consequence of the overprediction in 


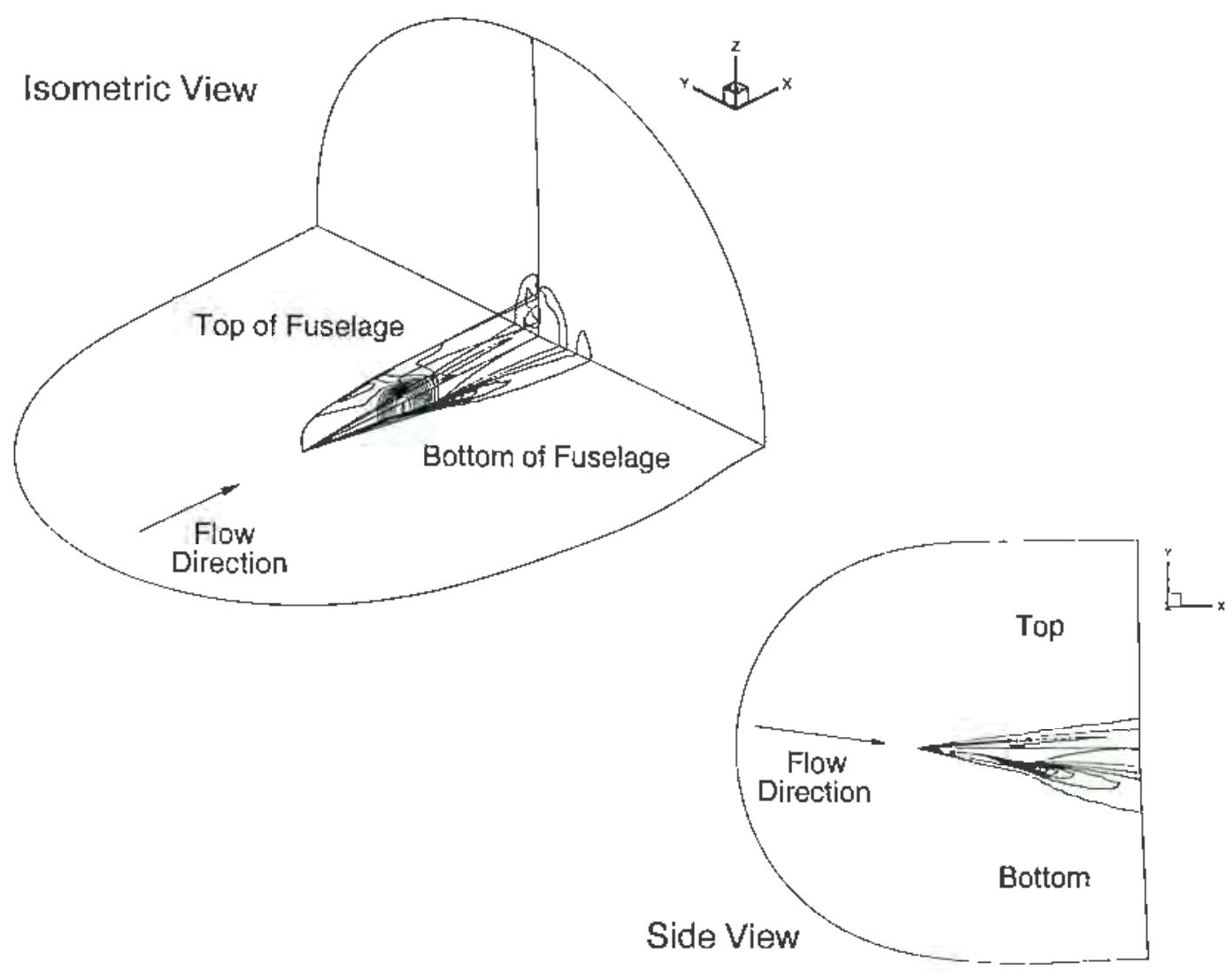

Figure 5.11: Normalized pressure distribution over Mach 12 vehicle fusclage $\left(M_{\infty}\right.$ $=9, \alpha=-3^{\circ}$.

pressure at the peak pressure location and along the leading edge of the forebody. However, the $\mathrm{L} / \mathrm{D}$ of the fuselage is underpredictod by only $2.2 \%$. Hence, the models accurately predict both the qualitative and quantitative performance of these fuselage geometries.

An isometric and side view of the computational solution for normalized pressure distribution over the Mach 12 vehicle at off-design conditions of $M=9$ and $\alpha=-3^{\circ}$ are shown in Fig. 5.11. A shock wave is now present on the upper surface of the fuselage because of the negative angle of attack incurred on the geometry. However, the angle of attack is low cnough that shock waves are formed on both the lower and upper surface of the fuselage. Olsserving the 


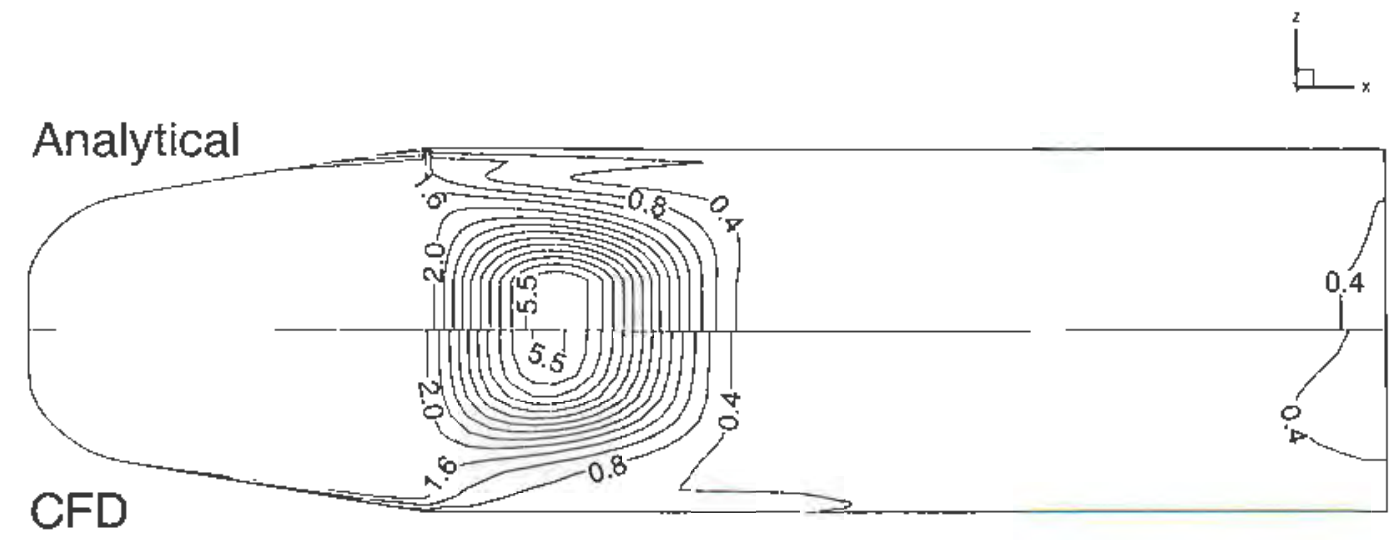

Figure 5.12: Normalized pressure distribution over lower surface of Mach 12 vohicle fuselage $\left(M_{\infty}=9, \alpha=-3^{\circ}\right)$.

base flow at the rear of the fuselage, the shock wave is again detached from the fusolage, but the separation distance is small.

The normalized pressure distribution on the lower surface of the Mach 12 vehicle at off-design conditions of $\mathrm{M}=9$ and $\alpha=-3^{\circ}$ is shown in Fig. 5.12. The forces calculated on both the lower and upper surface are found on the right side of Table 5.2. The lower surface flowfield is predicted quite well using the analytical methods developed above. The peak pressure on the fuselage is still being overpredicted, but the extent of the overprediction is smaller than the positive angle of attack case. Observing the forces recorded in Table 5.2, the lift and drag on the lower surface are overpredicted by $1.4 \%$ and $7 \%$, respectively. The magnitude of the thrust on the geometry is so small that the error in thrust prediction of $-32 \%$ is considered to be negligible.

The normalized pressure distribution on the upper surface of the Mach 12 vehicle at off-design conditions of $\mathrm{M}=9$ and $\alpha=-3^{\circ}$ is shown in Fig, 5.13. The forces calculated on the upper surface may be found on the right side of Talble 5.2. The most apparent alspect of Fig. 5.13 is the offect of cross-flow on the upper 


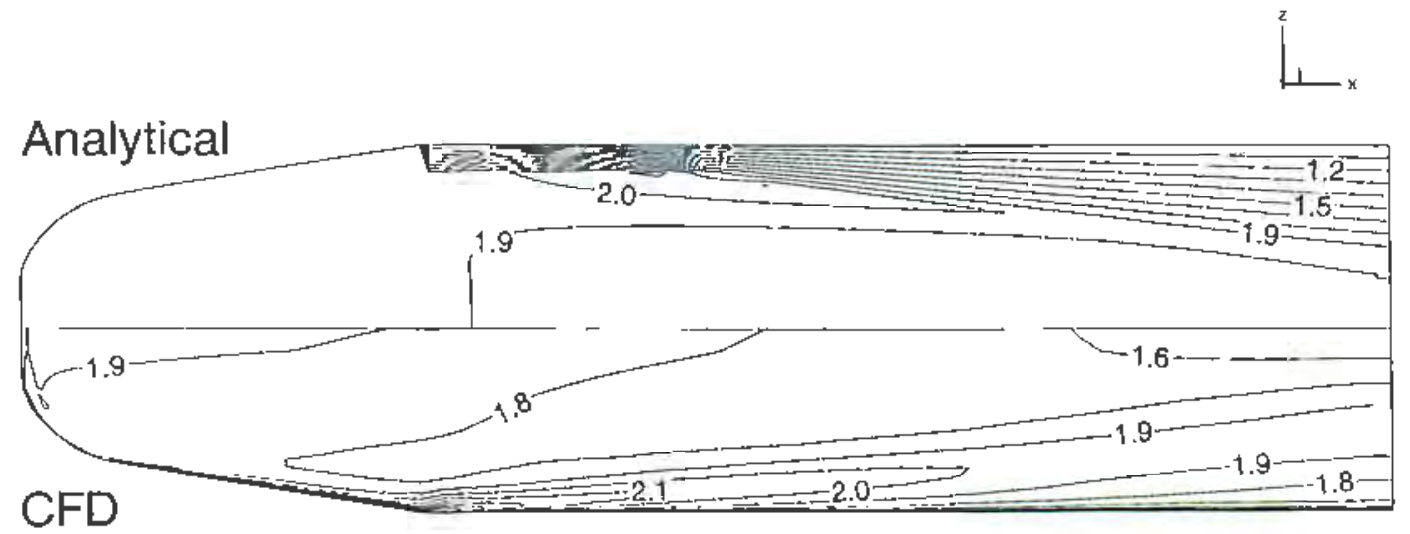

Figure 5.13: Nornalized pressure distribution over upper surface of Mach 12 vehicle fuselage $\left(M_{\infty}=9, \alpha=-3^{\circ}\right)$.

surface for the computational results. This effect was also shown for the upper surface of the fuselage for positive angle of attack (see Fig. 5.10). As stated before, cross-flow is ignored in this analysis, hence the three-dinensional effects observed in Fig. 5.13 are not captured. The lift and drag calculated on the upper surface have errors of $-1.5 \%$ and $-2.9 \%$, respectively. Hence, even though cross-flow is not modeled, the forces on the upper surface are predicted very well compared to the computational results.

The net lift and drag on the Mach 12 vehicle for $\mathrm{M}=9$ and $\alpha=3^{\circ}$ are shown in Table 5.2. The net lift on the fuselage is underpredicted by $5.5 \%$ and the net drag on the fuselage is overpredicted by $4.1 \%$. The lift to drag ratio of the geometry was found to be underpredicted by $9.2 \%$. Considering that all the errors are under $10 \%$, the models predict both positive and negative angle of attack for off-design Mach number quite well. 


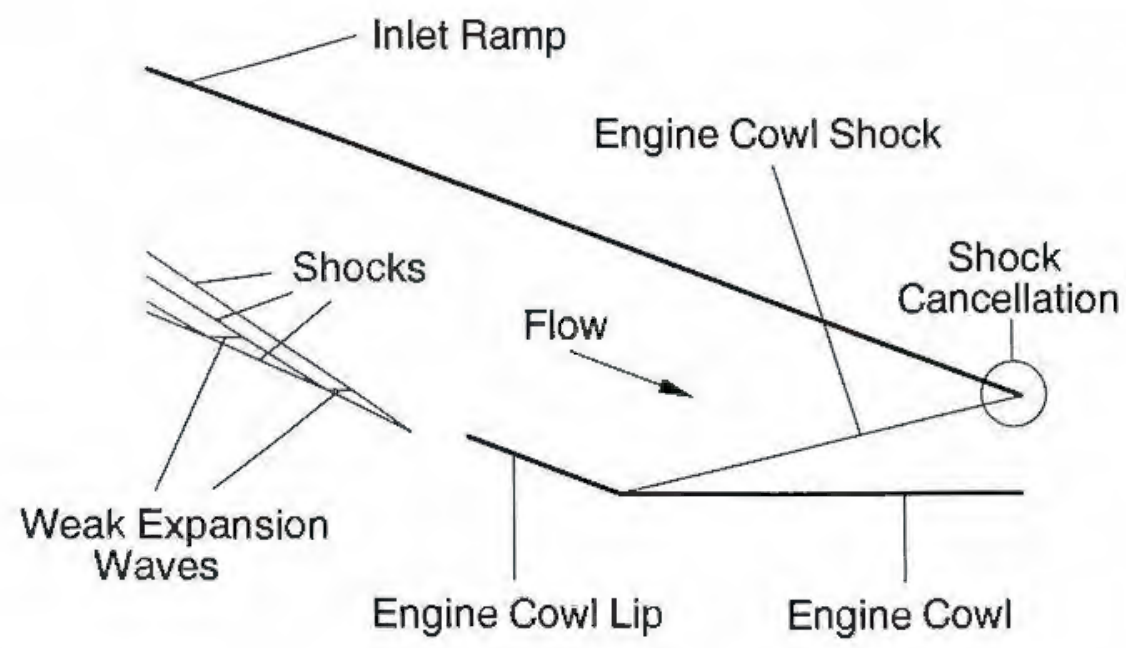

Figure 5.14: Inlet lip geometry.

\subsection{Off-Design Engine Cowl}

The shock waves off the forebody and the inlet ramps will shift for off-design angle of attack and Mach number. If not properly accounted, the shifted shock waves could enter into the engine, causing severe shock wave boundary layer interactions, localized heating, and boundary layer separation. Thus, a constraint is imposed upon the design such that the engine cowl is always positioned to prevent any shocks from being swallowed into the engine (any vehicle that violates the shock swallowing constraint is not considered). The engine cowl is allowed to translate axially to move the engine cowl shock such that it cancels on the upper surface of the engine. If an inlet interaction occurs such that an expansion off of the last shock interaction will enter the engine, a "lip" is added to the inlet to prevent any non-uniformities from entering the engine (see Fig. 5.14). The angle of the engine lip is the same as the flow off of the last inlet compression ramp. Thus, no shocks from the inlet lip enter into the engine (neglecting any shocks induced by boundary layers)

The inlet lip concept shown in Fig. 5.14 has some obvious limitations. The 
geometry will be difficult to manufacture and maintain. If the design is indeed mechanically feasible, the weight of the mechanism may be unacceptable. However, in light of other methods of tailoring the engine inlet flowfield such as moving the ramp inlets or translating the entire engine, the inlet lip seems to be a simple solution to the problem of flow uniformity entering the engine. 


\section{Chapter 6}

\section{Supersonic Off-Design Aerodynamic Performance}

The hypersonic modeling described in Chapter 5 will be shown in Chapter 7 to be effective along the entire hypersonic Mach regime. However, as the freestream Mach number is lowered below 5 , the hypersonic assumptions inherent, in shock-expansion theory, tangent-wedge, and tangent-cone flow becone invalid. As Mach number decreases, the slope of the flowfeld characteristics stcepens. Hence, characteristic reflections off of the bow shock produced by the vehicle's forcbody interact with more of the vehicle surface. The result is that hypersonic methods overpredict pressure for compressions. In this chapter, methods for predicting the pressure distribution in the supersonic regime $(1<\mathrm{M}<5-7)$ are developed to complete the modeling from transonic through hypersonic Mach number.

\subsection{Aerodynamic Model}

The acrodynamic model for the supersonic solutions is based on linearized, small perturbation, potential flow $43,56, \%$. The two-dimensional solution for the 
pressure coefficient $C_{p_{2 D}}$ using small perturbation, linearized potential flow is

$$
C_{p 2 D}=\frac{2 \theta}{\sqrt{M_{\infty}^{2}-1}}
$$

where 0 is defined in this work as the angle between the freestream flow direction and the local surface streamine (assumed to be two-dimensional, no cross-How). Equation 6.1 is applicable to both compression and expansion so long as tho pressure coefficient is not bolow the minimum pressure coefficient found at zero pressure

$$
C_{p_{\min }}=-\frac{2}{\gamma M_{\infty}^{2}}
$$

Thus, for any given geometry, there can be no expansion angle greater than

$$
\theta_{\mathrm{milt}}=\frac{\sqrt{M_{\infty}^{2}-1}}{\gamma M_{\infty}^{2}}
$$

If an cxpansion angle greater than $\theta_{\min }$ occurs on the fuselage relative to fresstream, the pressure on the surface is assumed to be zero. Equation 6.1 is used on all expansion surfaces and all compression surfaces that see a decrease in local inclination (i.e, for positive angle of attack use Eq. 0.1 on the upper surface; for negative angle of attack use Eq. 6.1 on the lower surface).

For angle of attack calculations, the cross-flow occurring on the compression surfaces of the geometry is significant enough that the assumption of twodimensional flow substantially overpredicts the pressure. For these cases, the pressure coefficients on all compression surfaces that have an increase in local inclination (i.e., for positive angle of attack on the lower surface; for negative angle of attack on the upper surface) are calculated using the linearized, small perturbation, potential flow solution on a cone ${ }^{77}$

$$
C_{p_{c}}=-2 \tan ^{2} \theta \ln \left(\frac{1-\sqrt{1-\left(M_{\infty}^{2}-1\right) \tan ^{2} \theta}}{\tan \theta \sqrt{M_{\infty}^{2}-1}}\right)
$$


For the conical solution, the maxinum inclination angle can be found from Eq. 6.4

$$
\theta_{\text {max }}=\arctan \left(\frac{1}{\sqrt{M_{\infty}^{2}-1}}\right)
$$

For compression inclination angles that exced $\theta_{\max }$ defined by En. 6.5 , the twodimcmsional results of Eq. 6.1 are used.

Another obvious means of prodicting the compression flowfield on the fuselage is to use tangent-wedge and/or tangent-cone flow. As mentioned above, these methods aro more applicable in hypersonic flow. Preliminary studies using tangent-wedge flow found that pressures wore overpredicted, thus significantly overpredicting the lift and drag on these geometries. Tangent-cone flow was not investigated, but it is assumed that this mothodology would have similar results to Eq. 6.4.

\subsection{Validation}

Figure 6.1 shows an isometric and side view of the computational solution for normalized pressure distribution over the Mach 12 vehicle at off-design conditions of $\mathrm{M}=3$ and $\alpha=3^{\circ}$. The shock waves on the geometry are pushed off of the fuselage due to lower freestream Mach number. An expansion occurs on the upper surface from the positive angle of attack incurred on the vehicle. Observing the base plane at the ond of the vehicle fusclage, the shock wave is detached from the fusclage side edge, and has a large detachment distance.

The nornalized pressure distribution on the lower surface of the Mach 12 vehiclo at off-design conditions of $\mathrm{M}=3$ and $\alpha=3^{\circ}$ is slown in Fig. 6.2. The forces calculated on both the lower and upper surface are found on the left side of Table 6.1. Overall, the supersonic analytical model is less accurate than the hypersonic analytical model. The expansion flowfidd is modeled rather woll, 


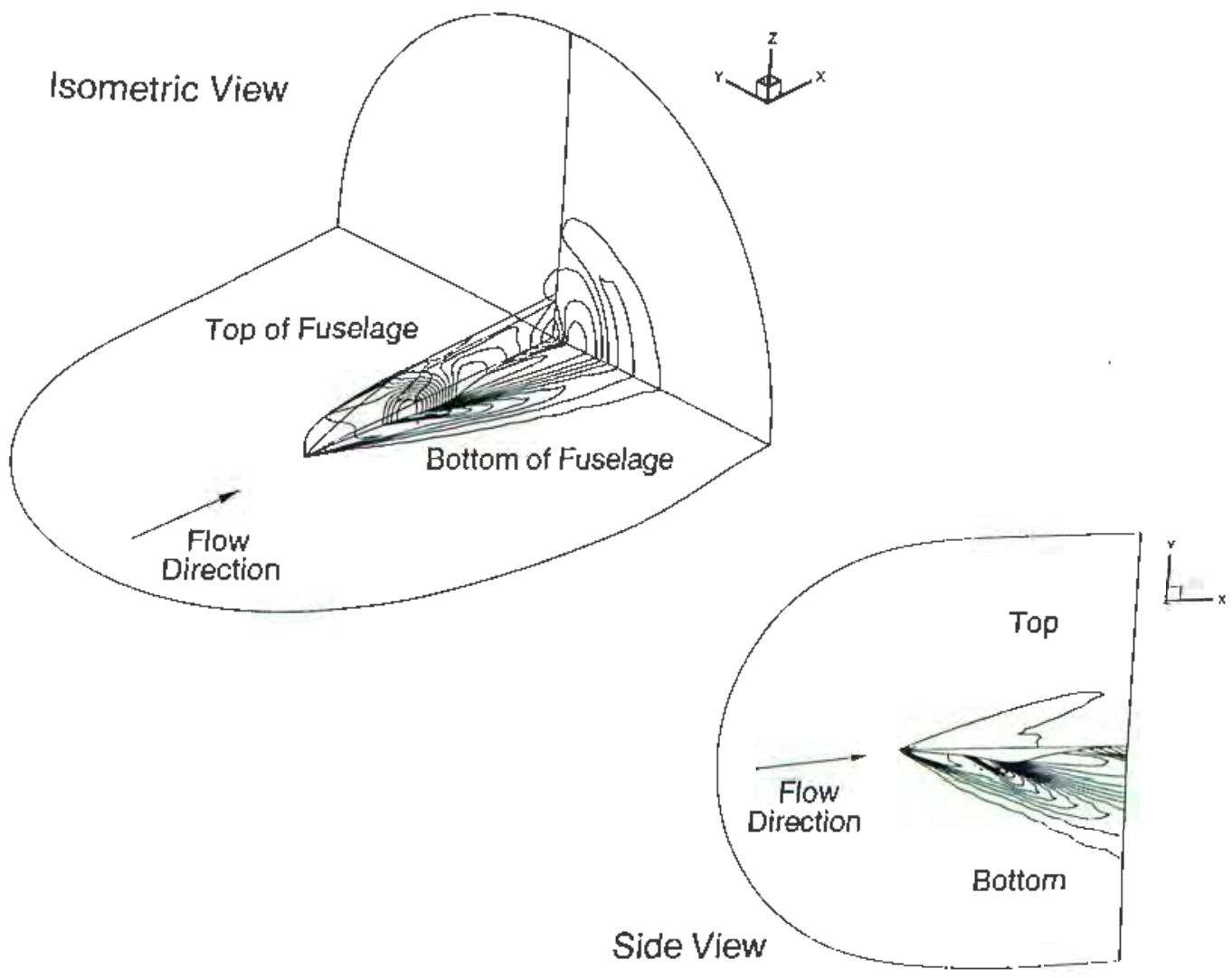

Figure 6.1: Normalized pressure distribution over Mach 12 vehicle fuselage ( $M_{\infty}$ $\left.=3, \alpha=3^{0}\right)$. 
Analytical
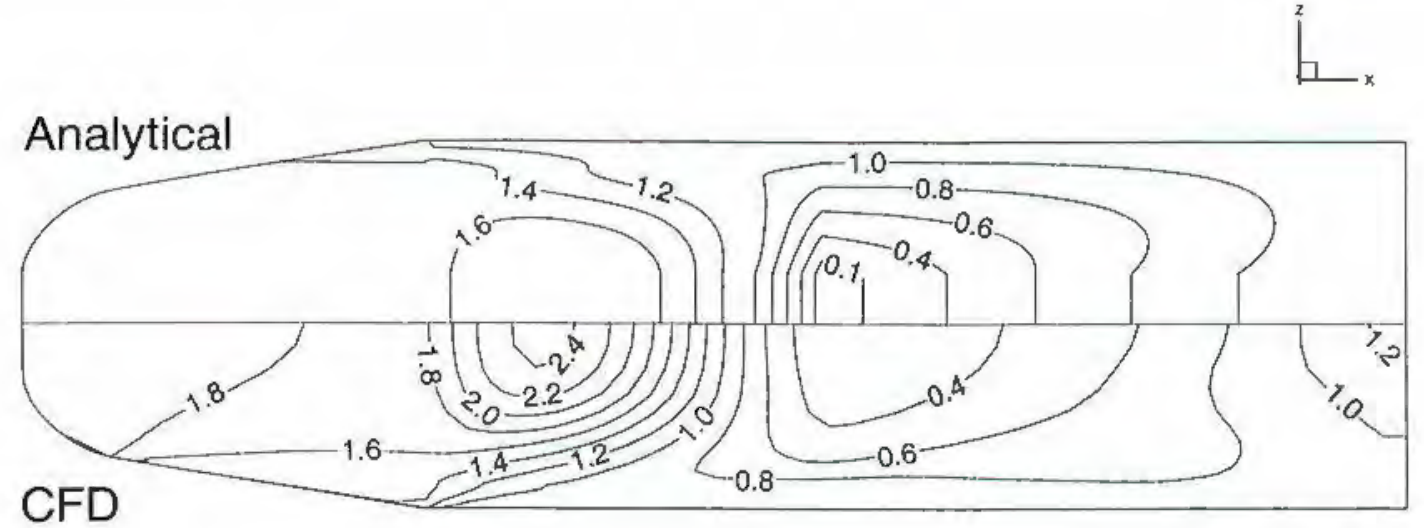

Figure 6.2: Normalized pressure distribution over lower surface of Mach 12 vehicle fuselage $\left(M_{\infty}=3, \alpha=3^{\circ}\right)$.

but the compression flowfield underpredicts the pressure, with an error in peak pressure of approximately $-30 \%$. The lift and drag on the lower surface have errors of $-2.5 \%$ and $-13.8 \%$, respectively.

The normalized pressure distribution on the upper surface of the Mach 12 vehicle at off-design conditions of $\mathrm{M}=3$ and $\alpha=3^{\circ}$ is shown in Fig. 6.3. The forces calculated on the upper surface are found on the left side of Table 6.1. Crossflow over the upper surface is significant and substantially alters the flowheld compared to the analytical model. The lift and thrust on the upper surface have errors of $-10.6 \%$ and $-11.5 \%$, respectively. These errors are a direct consequence of assuming no cross-flow in the supersonic model.

The net lift and drag on the Mach 12 vehicle at $\mathrm{M}=3$ and $\alpha=3^{\circ}$ are recorded in Table 6.1. Again, the supersonic model tends to be less accurate in force prediction compared to the hypersonic model. The net lift was overpredicted by $26 \%$ and the net drag was underpredicted by $21 \%$, causing an overprediction in $\mathrm{L} / \mathrm{D}$ of $61 \%$. These errors are caused by the underprediction of negative lift on the upper surface. Hence, the assumption of no cross-flow has a significant effect 


\begin{tabular}{|c|c|c|c|c|}
\hline & \multicolumn{2}{|c|}{$\alpha=3^{\circ}$} & \multicolumn{2}{c|}{$\alpha=-3^{\circ}$} \\
\cline { 2 - 5 } & Analytical & CFD & Analytical & CFD \\
\hline$L_{\text {bot }}$ & 800 & 829 & 573 & 636 \\
$D_{\text {tot }}$ & 83 & 97 & 27 & 26 \\
$T_{\text {bot }}$ & 10 & 9 & 17 & 29 \\
$\% L_{\text {bot }}$ & -2.5 & - & -9.9 & - \\
$\% D_{\text {toot }}$ & -13.8 & - & 2.1 & - \\
$\% T_{\text {tot }}$ & 14.4 & - & -42.1 & - \\
\hline$L_{\text {top }}$ & -576 & -645 & -813 & -845 \\
$D_{\text {top }}$ & 0 & 0 & 45 & 46 \\
$T_{\text {top }}$ & 28.8 & 33 & 0 & 0 \\
$\% L_{\text {top }}$ & -10.6 & - & -3.7 & - \\
$\% D_{\text {top }}$ & 0 & - & -3.2 & - \\
$\% T_{\text {top }}$ & -11.5 & - & 0 & - \\
\hline$L_{\text {tot }}$ & 233 & 185 & -240 & -209 \\
$D_{\text {tot }}$ & 44 & 56 & 55 & 43 \\
$L / D$ & 5.3 & 3.3 & -4.4 & -4.9 \\
$\% L_{\text {tot }}$ & 25.9 & - & 14.8 & - \\
$\% D_{\text {tot }}$ & -21.1 & - & 27.9 & - \\
$\% L / D$ & 60.6 & - & -10.2 & - \\
\hline
\end{tabular}

Table 6.1: Forces on Mach 12 vehicle design at $\mathrm{M}=3\left(\mathrm{~m}^{2}\right)$. 
Analytical
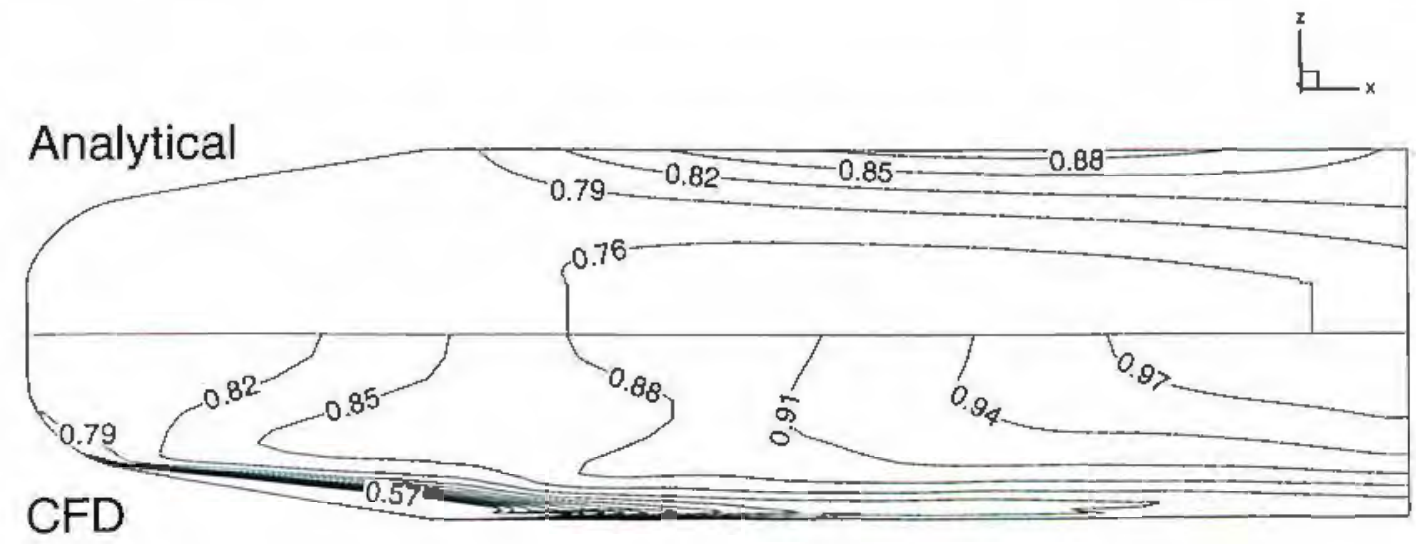

Figure 6.3: Normalized pressure distribution over upper surface of Mach 12 vehicle fuselage $\left(M_{\infty}=3, \alpha=3^{\circ}\right)$.

on force calculation.

Figure 6.4 shows an isometric and side view of the computational solution for normalized pressure distribution over the Mach 12 vehicle at off-design conditions of $\mathrm{M}=3$ and $\alpha=-3^{\circ}$. A shock wave exists on the upper surface of the fuselage from the negative angle of attack incurred on the vehicle. The shock wave has a large detachment distance from the fuselage side edge as well as shock waves pressed substantially off the body due to the low Mach number.

The normalized pressure distribution on the lower surface of the Mach 12 vehicle at off-design conditions of $\mathrm{M}=3$ and $\alpha=-3^{\circ}$ is shown in Fig. 6.5. The forces calculated on both the lower and upper surface may be found on the right side of Table 6.1. Again, the supersonic model was less accurate than the hypersonic model, however the negative angle of attack solution modeled the flowfield better than the solution for positive angle of attack (Fig. 6.2). The lift and drag errors on the lower surface were calculated to be $-9.9 \%$ and $2.1 \%$, respectively. However, the thrust error $(-42 \%)$ was found to be substantial, and was likely due to the significant underprediction of pressure on the rear of the 


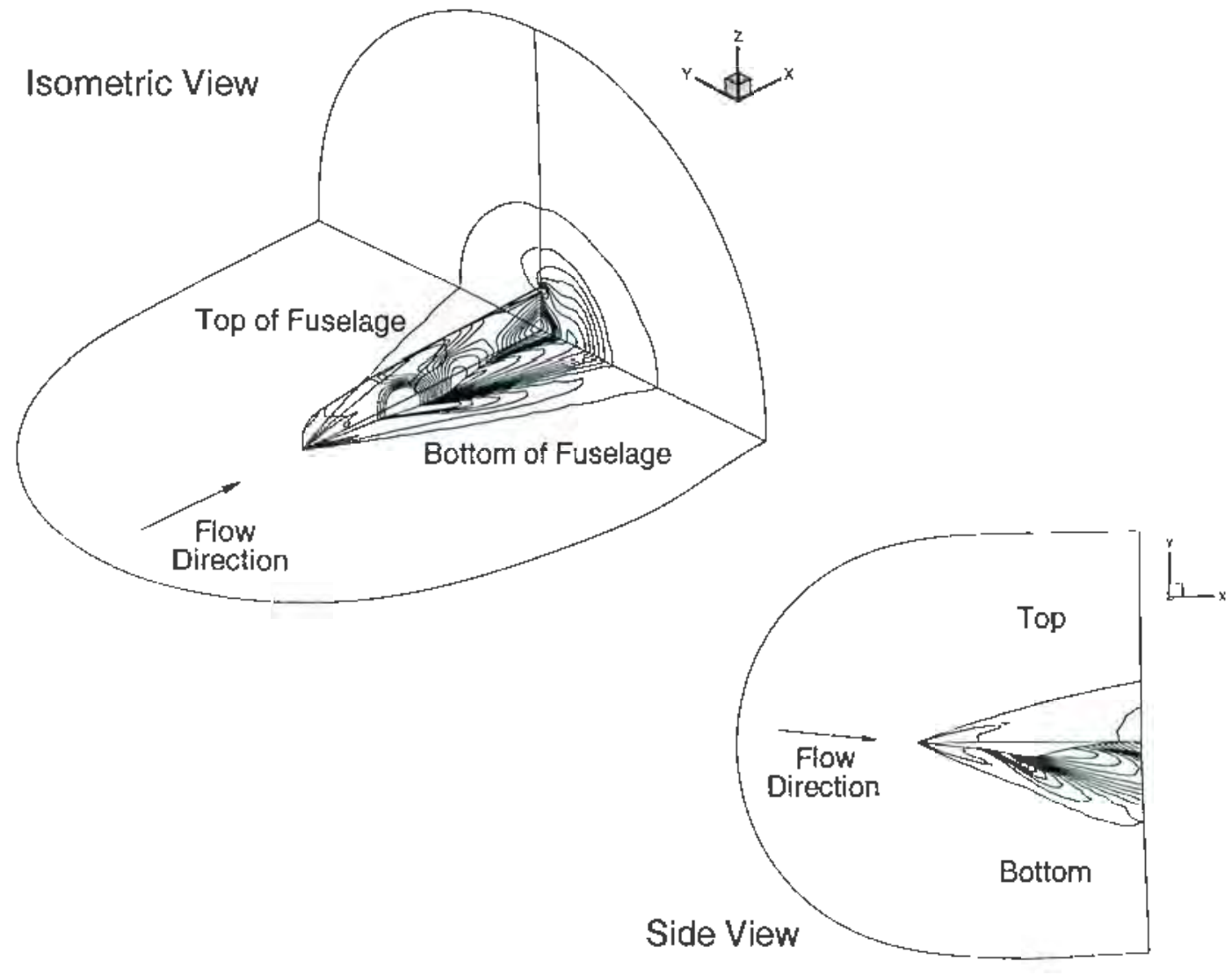

Figure 6.4: Normalized pressure distribution over Mach 12 vehicle fuselage $\left(M_{\infty}\right.$ $\left.=3, \alpha=-3^{\circ}\right)$. 


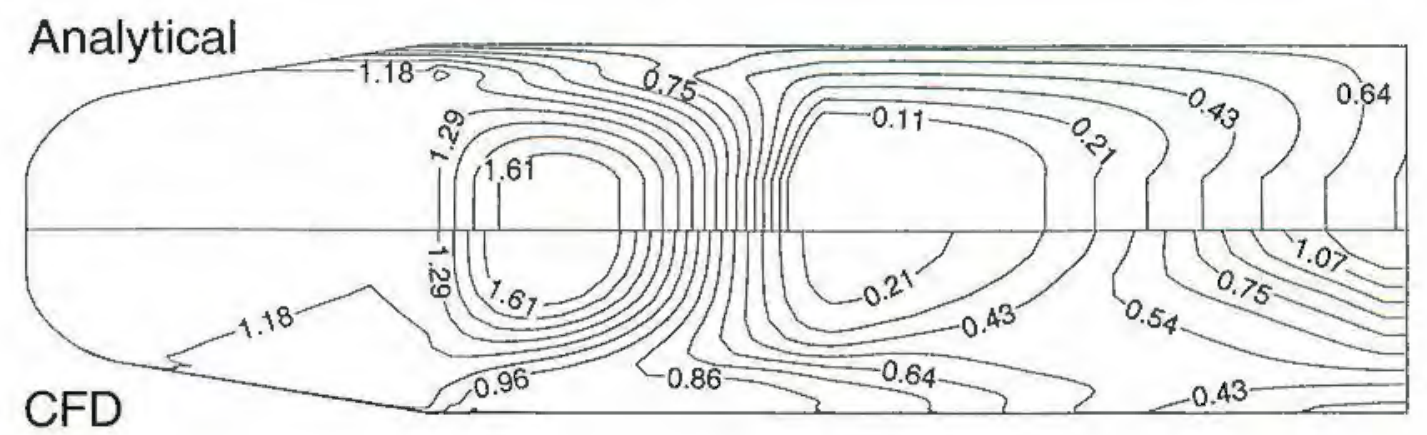

Figure 6.5: Normalized pressure distribution over lower surface of Mach 12 vehicle fuselage $\left(M_{\infty}=3, \alpha=-3^{\circ}\right)$.

fuselage.

The normalized pressure distribution on the upper surface of the Mach 12 vehicle at off-design conditions of $\mathrm{M}=3$ and $\alpha=-3^{\circ}$ is shown in Fig. 6.6. The forces calculated on the upper surface are found on the right side of Table 6.1. Cross-flow is again seen to be significant, substantially altering the upper surface flowfield. Even though the model does not qualitatively model the upper surface, it does predict the lift to within $-3.7 \%$ and the drag to within $-3.2 \%$ compared to the computational results.

The net lift and drag on the Mach 12 vehicle at $\mathrm{M}=3$ and $\alpha=-3^{\circ}$ is calculated in Table 6.1. The net lift and drag are overpredicted by $14.8 \%$ and $27.9 \%$, respectively compared to the computational results. The resulting error in $\mathrm{L} / \mathrm{D}$ was found to be $-10.2 \%$. As noted above, the effect of cross-flow is clear for the supersonic solutions. However, the error in lift can be attributed almost equally to model errors on both the upper and lower surfaces. 


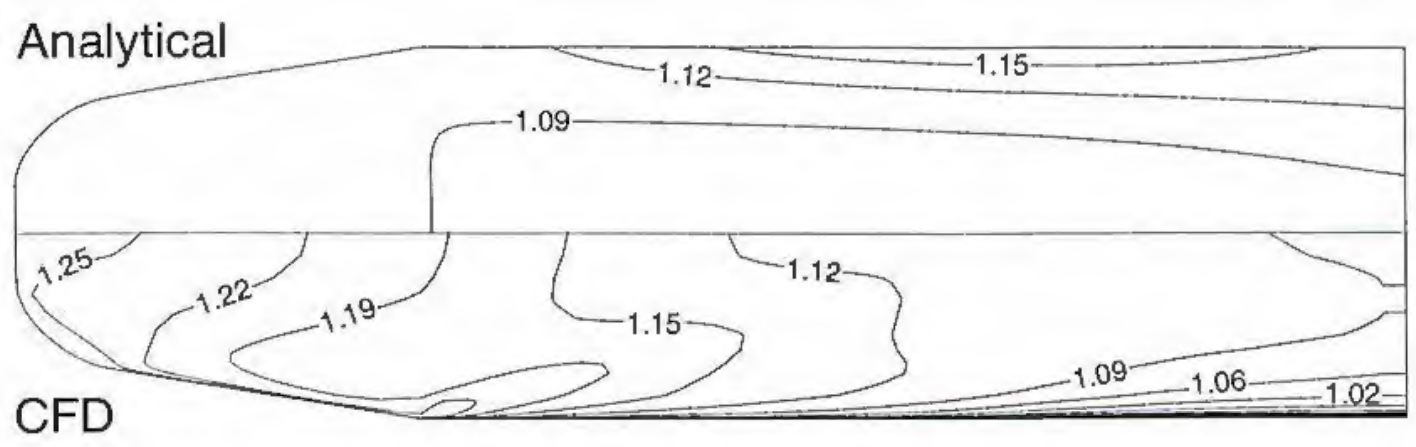

Figure 6.6: Normalized pressure distribution over upper surface of Mach 12 vehicle fuselage $\left(M_{\infty}=3, \alpha=-3^{\circ}\right)$.

\subsection{Off-Design Engine Cowl}

The supersonic engine cowl is handled in the same manner as the hypersonic engine cowl. The inlet lip is unnecessary at supersonic velocity due to higher shock angles. Thus, the only engine cowl requirement is retaining shock cancellation on the upper surface of the vehicle at the engine inlet. It is assumed that supersonic flow always exists following the shock cancellation. Thus, transonic effects on engine inlet performance are ignored. 


\section{Chapter 7}

\section{Results}

The analytical models developed in Chapters $2-6$ are now applied to a vehicle design for a large range of Mach numbers and angles of attack. The first section involves the pure airframe (engine removed) only. This analysis assesses the accuracy of the off-design models developed in Chapters 4-6 and establishes limits of application. The second section presented is the scramjet-powered mode of the RBCC engine for the full vehicle. This analysis demonstrates the effects of Mach number and angle of attack on a full-powered, scramjet-mode vehicle. The third section applies the full-powered vehicle to a trajectory analysis to demonstrate the ability to apply the models developed herein to applications that require aerodynamic information.

\subsection{Unpowered Fuselage Performance}

\subsubsection{Hypersonic Performance}

A series of computational solutions were performed on the Mach 12 vehicle geometry presented in Chapter 5 for a range of Mach numbers between 3 and 15 and a range of angles of attack between $-3^{\circ}$ and $6^{\circ}$. The computational grid 


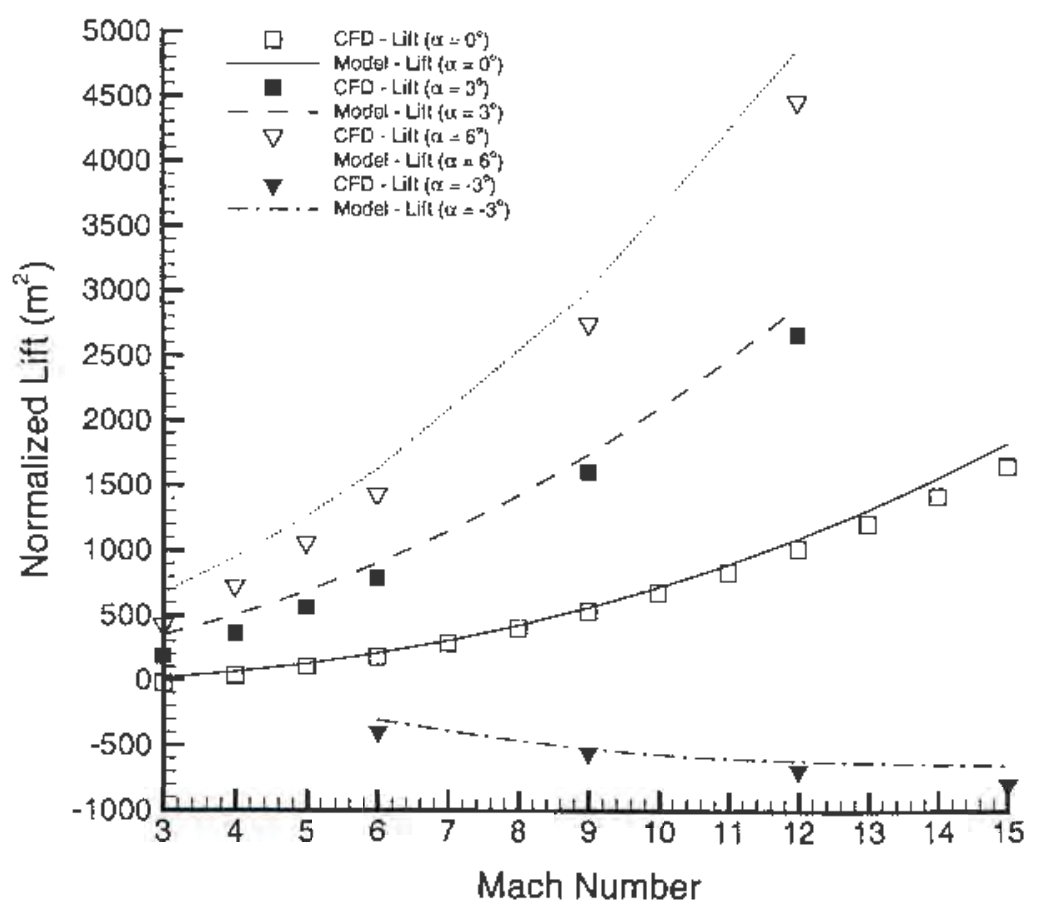

Figure 7.1: Hypersonic lift $\left(\mathrm{m}^{2}\right)$ of Mach 12 vehicle as a function of Mach number.

used for all the hypersonic calculations is the same grid presented in Fig. 4.28 with the outer box grid and the wake region removed. OVERFLOW 74 is used to solve the $3-\mathrm{D}$, inviscid flowfield over the fuselage. The computational results are then compared to the hypersonic off-design models presented in Chapter 5.

The normalized, inviscid lift of the geometry in units of $\mathrm{m}^{2}$ (the forces are calculated using a non-dimensionalized pressure $\left.p / p_{\infty}\right)$ is plotted in Fig. 7.1 along with the computational results. Overall, the hypersonic model accurately predicts the qualitative trend in lift for the full Mach number and angle of attack range. Percent errors increase as Mach numher decreases, consistent with the violation of hypersonic assumptions at lower speeds. The models tend to overpredict lift for all Mach numbers and angles of attack solved, consistent with the overprediction of pressure in the peak compression region on the geometry. The 


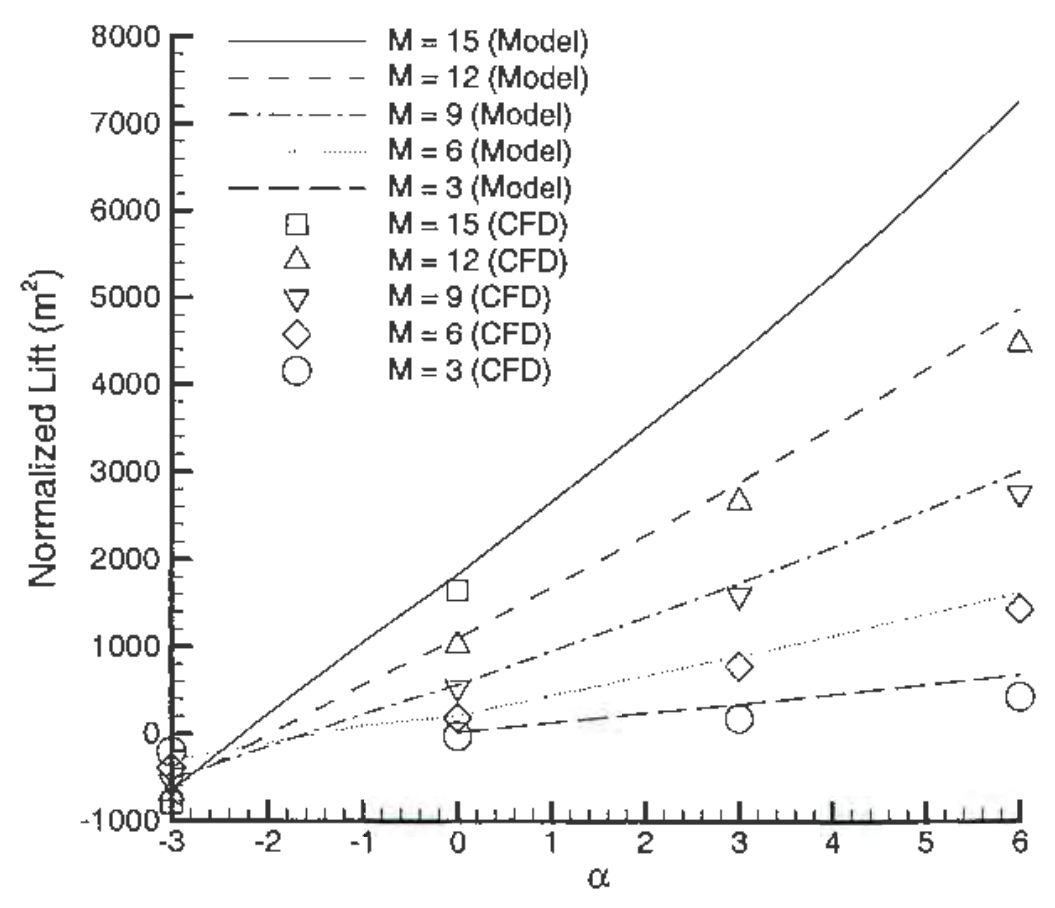

Figure 7.2: Hypersonic lift $\left(\mathrm{m}^{2}\right)$ of Mach 12 vehicle as a function of angle of attack.

lift was found to drastically decrease with Mach number (the zero-lift Mach number for zero angle of attack was 3.5), indicating that low-speed lift perfornance may be an issue. The lower limit of application for the hypersonic model was between Mach 5 and 6, where hypersonic assumptions begin to become invalid.

The normalized, inviscid lift of the geometry is plotted as a function of angle of attack along with the computational solutions in Fig. 7.2. For all Mach numbers and angles of attack solved, the lift was almost lincar with respect to angle of attack, deviating from linear near zero angle of attack. The lift-curve slope was seen to decrease with Mach number, alınost approaching zero by Mach 3. The zcro-lift angle of attack for all the hypersonic solutions was below zcro, while the zero-lift angle of attack for the Mach 3 solution was betwecn $1^{\circ}$ and 


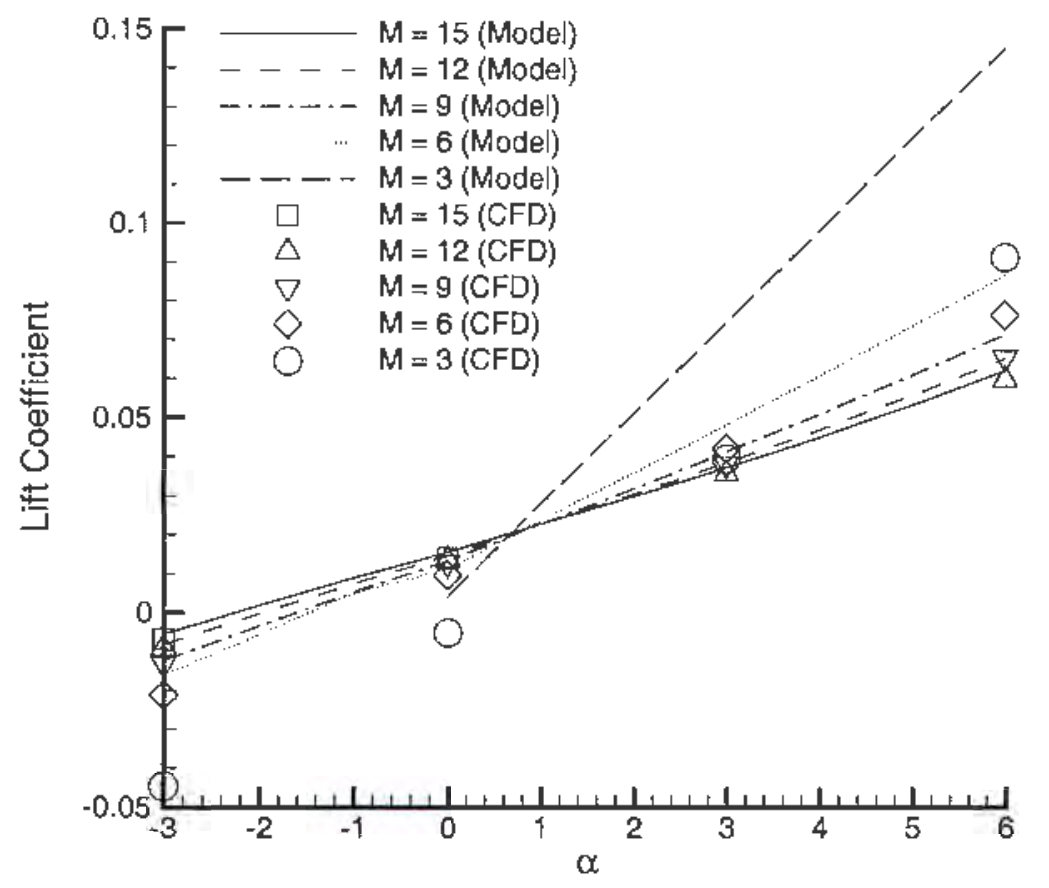

Figure 7.3: Hypersonic lift coeflicient of Mach 12 vehicle as a function of angle of attack.

$2^{\circ}$.

The inviscid lift coefficient of the geometry is plotted as a function of angle of attack along with the computational solutions in Fig. 7.3. The lift coefficient lias a linear profile with respect to angle of attack for all Mach numbers solved. The solution for Mach 3 has the largest crror, showing that the hypersonic model cannot be applied at this Mach number. The lift coefficient displays hypersonic cquivalence at the higher Mach numbers, being roughly equivalent for the Mach 12 and 15 solutions. The lift-curve slope is observed to increase with decreasing Mach xumber (the opposite trend compared to the lift-curve slope in Fig. 7.2).

The normalized, inviscid drag of the geometry is plotted in Fig. 7.4 along with the computational results. The hypersonic model was found to accurately predict 


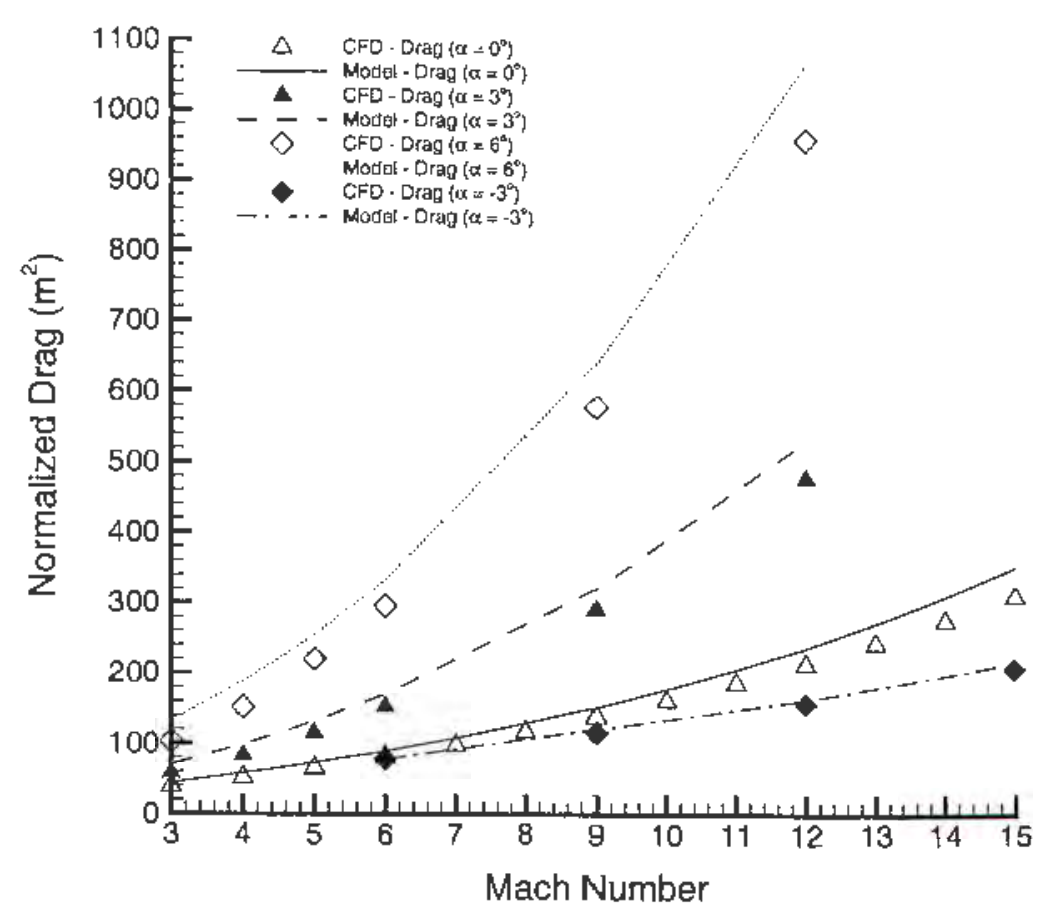

Figure 7.4: Hypersonic, inviscid drag $\left(\mathrm{m}^{2}\right)$ of Mach 12 vehicle as a function of Mach number.

the trend in drag for the entire Mach number and angle of attack range solved. As with the lift, percent errors increased with a decrease in Mach number due to the violation of hypersonic assumptions. The drag was found to be overpredicted for all the cascs solved because of the overprediction of peak pressure. Drag was found to decrease with Mach number and angle of attack, with the minimum occurring below zero angle of attack for a given Mach number.

The normalized, inviscid drag of the geometry is plotted as a function of angle of attack along with the computational solutions in Fig. 7.5. For all Mach numbers and angles of attack solved, the models qualitatively match the trends. A minimun in drag was found for the Mach 12,9 , and 6 solutions at approximately $-2^{\circ}$. 'The drag minimum is found where the angle of attack roughly balances the 


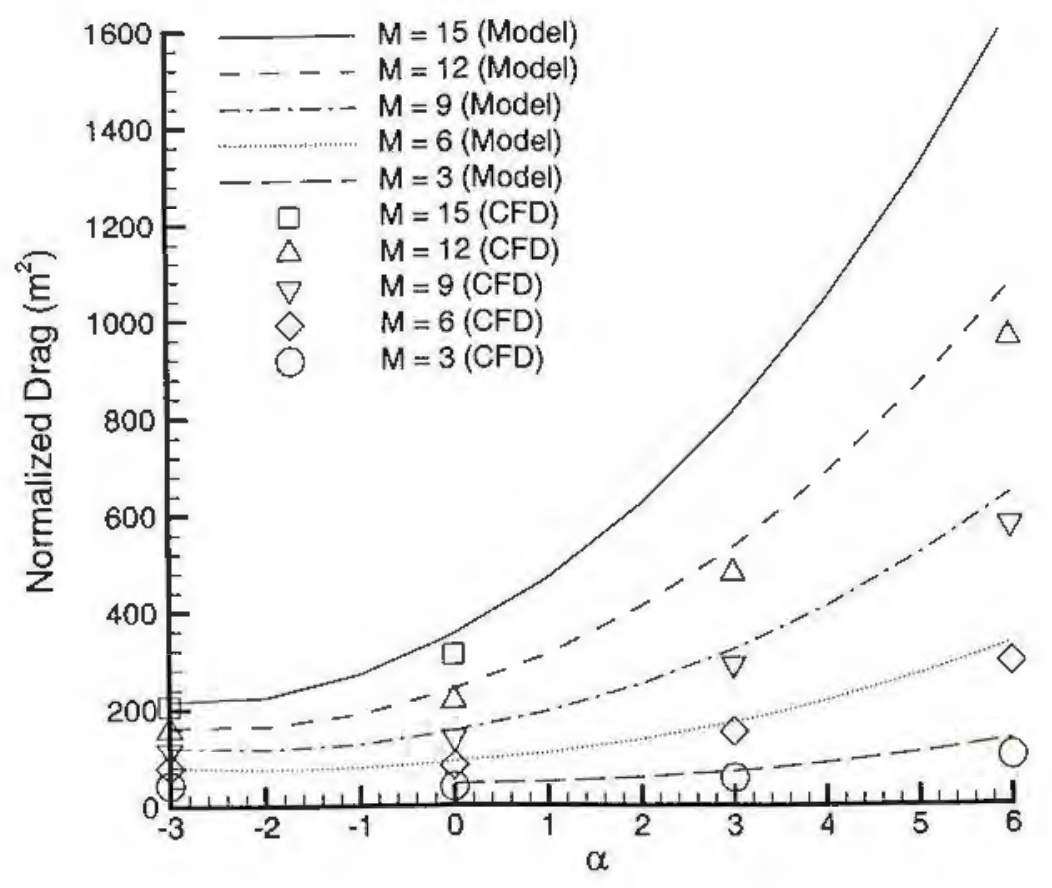

Figure 7.5: Hypersonic, inviscid drag $\left(\mathrm{m}^{2}\right)$ of Mach 12 vehicle as a function of angle of attack.

compression occurring on both the lower and upper surface.

The inviscid drag coefficient of the geometry is plotted as a function of angle of attack along with the computational solutions in Fig. 7.6. The drag coefficient is seen to increase with decreasing Mach number. The Mach 3 solution is again shown to be inaccurate, hence the hypersonic model cannot be applied at this Mach number. For the rest of the solutions, the model captures the qualitative trends and predicts minimum drag location consistent with the computational solutions.

The hypersonic, inviscid drag polar of the vehicle at several Mach numbers is plotted in Fig. 7.7 along with the computational solutions for lift and drag coefficient. As noted in Figs. 7.3 and 7.6, the Mach 3 solution using the hy- 


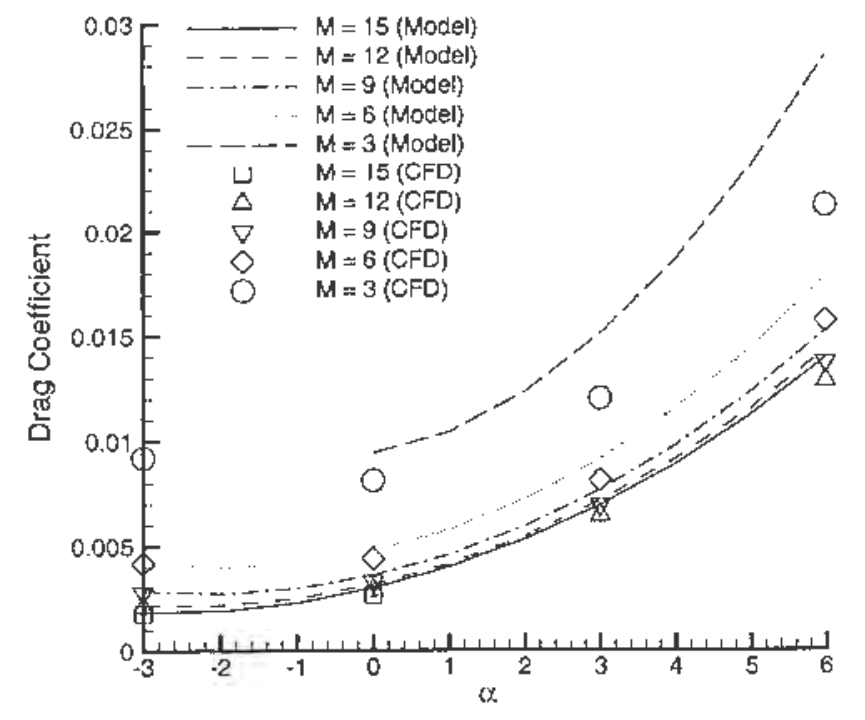

Figure 7.6: Hypersonic, inviscid drag coefficient of Mach 12 vehicle as a function of angle of attack.

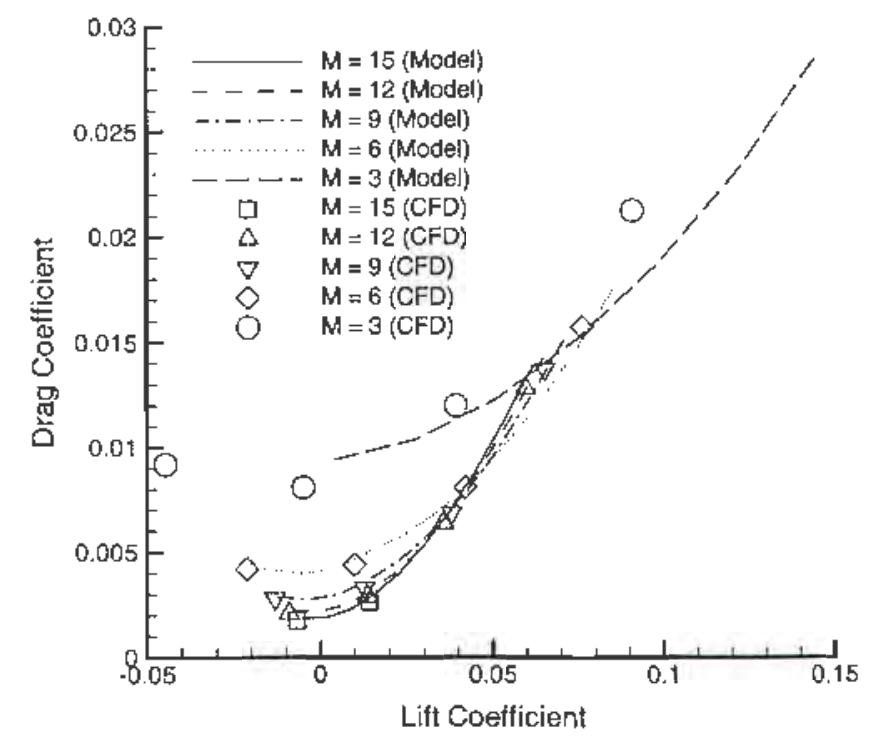

Fignre 7.7: Hypersonic, inviscid drag polar of Mach 12 vehicle. 


\begin{tabular}{|c|c|c|c|c|}
\hline$M_{\infty}$ & $m_{1}$ & $m_{2}$ & $m_{3}$ & $R$ \\
\hline 15 & 0.00186 & 1.4057 & 1.7119 & 0.99998 \\
12 & 0.00222 & 1.3488 & 1.7266 & 0.99999 \\
9 & 0.00291 & 1.2335 & 1.7455 & 0.99997 \\
6 & 0.00428 & 0.9702 & 1.7489 & 0.99978 \\
\hline
\end{tabular}

'Table 7.1: Hypersonic, inviscid drag' polar curve fit coefficients.

personic model is not accurate. Again, hypersonic cquivalence for the Mach 12 and 15 solutions is observed for the range of angles of attack solved. The Mach 9 solution is also found to be close to the Mach 12 and 15 solutions. Overall, the models capture the trends of the drag polar curve for Mach number ranging from 6 to 15 .

The hypersonic, inviscid drag polars plotted in Fig. 7.7 may be expressed in the following notation:

$$
C_{t}=m_{1}+m_{2} C_{l}^{m_{3}}
$$

Equation 7.1 is in the same general form that the drag coefficient is usually expressed:

$$
C_{d}=C_{d_{o}}+K C_{l}^{n t}
$$

where the drag cocfhicient is broken into a viscous term $\left(C_{d_{0}}\right)$ and a lift term $\left(K C_{l}^{n}\right)$. Since the above analysis is inviscid, $m_{1}$ in Eq. 7.1 is not the same as $C_{d_{b}}$. However, $m_{2}$ and $m_{3}$ are directly related to $K$ and $n$, respectively. The drag polars plotted in Fig. 7.7 that had positive lift coefficient were curve fitted using Eq. 7.1. The resulting values for the coefficients $m_{1}, m_{2}$, and $m_{3}$ are tabulated in Table 7.1 along with the curve fit accuracy $R$. Observing the cxponent $m_{3}$, all the results had values near 1.75 . This value is the average of the exponent found 


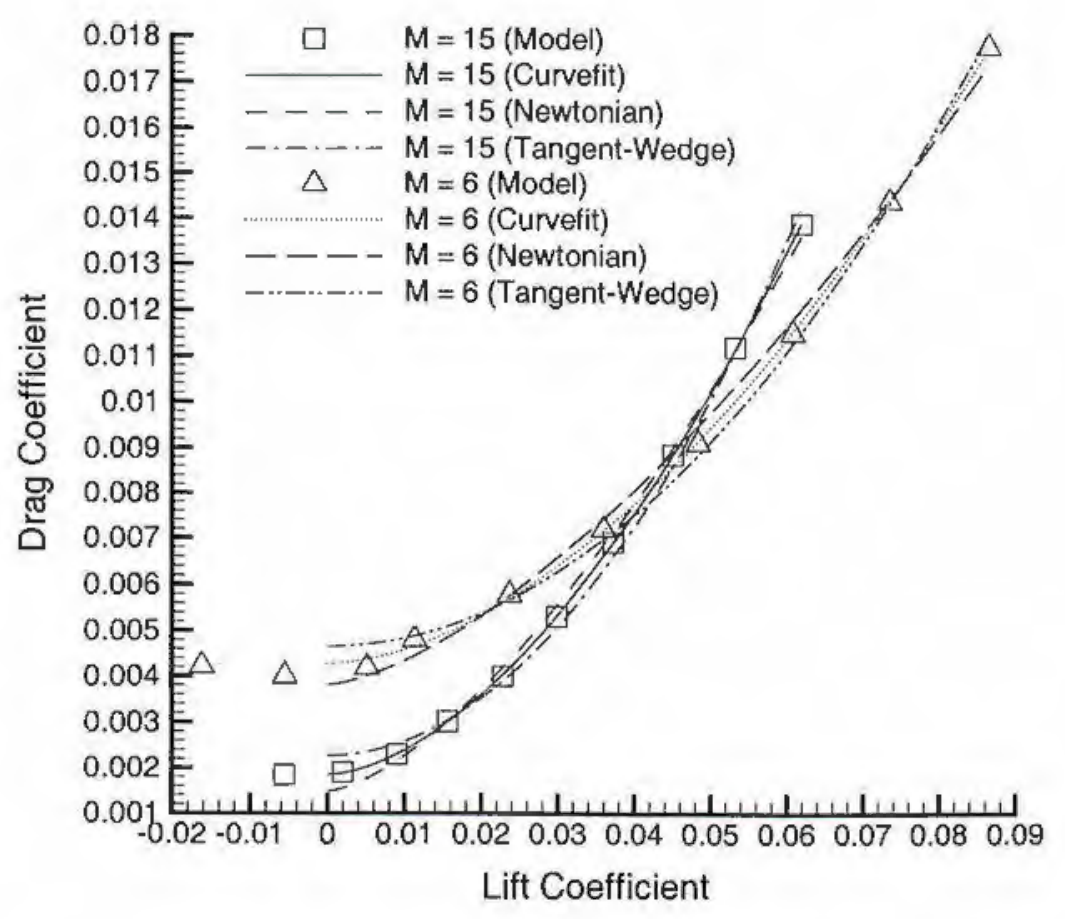

Figure 7.8: Comparison of curve fits to Newtonian and tangent-wedge flow.

from Newtonian flow (1.5) and tangent-wedge flow (2), and has been previously demonstrated by experiment ${ }^{26}$ to model hypersonic waverider behavior at angle of attack. The value of $m_{3}$ is seen to have a slight Mach number dependency, decreasing with increasing Mach number. The trend of $m_{3}$ may indicate that as Mach number goes to infinity the coefficient tends toward the Newtonian flow limit of 1.5. As Mach number goes to infinity (and $\gamma$ goes toward 1 ), the oblique shock relations are equivalent to Newtonian flow. Thus, the trend in $m_{3}$ with respect to Mach number is consistent with hypersonic flow and Newtonian flow theory.

Figure 7.8 compares the curve fits for the inviscid drag polars of the Mach 6 and 15 solutions to the curve fits using the Newtonian flow exponent of 1.5 and the tangent-wedge exponent of 2 . The curve fits using the exponents in 
Table 7.1 give excellent results compared to the hypersonic model data. The Newtonian flow and tangent-wedge flow results are seen to lie close to the clata, thus showing that either method would give a good approximation to the results from the hypersonic model.

\subsubsection{Supersonic Performance}

A series of computational solutions were performed on the Mach 12 vehicle geonetay presented in Chapter 6 for a range of Mach numbers between 1.21 and 6 and a range of angles of attack between $-3^{\circ}$ and $6^{\circ}$. The computational grid used for all the supersonic calculations is the same grid presented in Figs. 4.28 and 4.29. OVERFLOW ${ }^{\text {th }}$ is used to solve the 3-D, inviscid flowfield over the fuselage. The computational results are then compared to the supersonic offdesign models presontod in Chapter 6.

The normatized, inviscid lift of tho geometry is plotted in Fig. 7.9 along with the computational results. The supersonic model tonds to work well for Mach numbers between 3 and 6, with substantial errors in lift below Mach 3. Although the supersonic model captures the basic trend of lift reduction with a decrease in Mach ummber, the model does not have a smooth profile in the vicinity of Macl 3. Recalling Eq. 6.5, the limits for the axisymmotric method for positive angle of attack wero reached for Mach numbers greater than 4. For all angles of attack solved, the lift is seen to drastically decrease with decreasing Mach number. Hence, although the supersonic model does not accurately predict the lift at low Mach numbers, it does predict that lift will be low. This is an important conclusion when considering the low-speed aspects of the vehicle design and trajectory.

Comparing the supersonic results with the results of the hyporsonic model, it was found that the supersonic model had much higher errors in the prediction of 


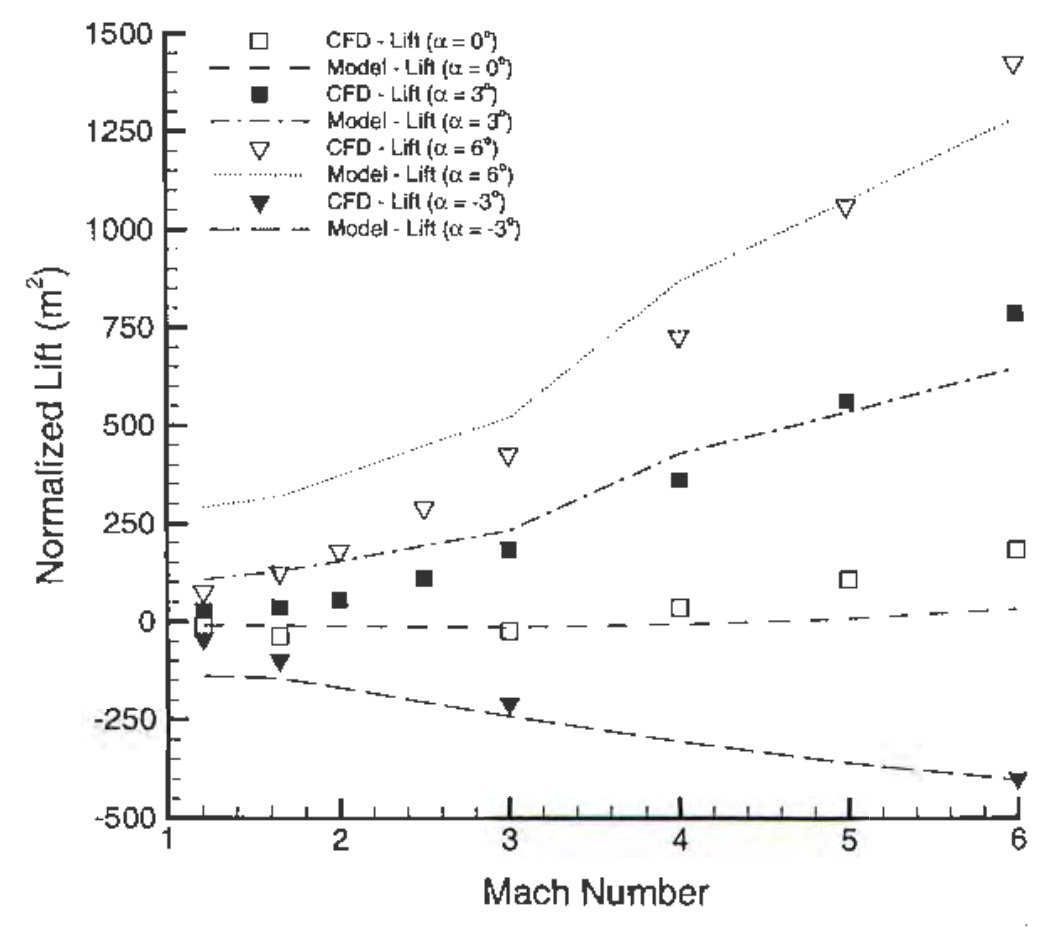

Figure 7.9: Supersonic lift $\left(\mathrm{m}^{2}\right)$ of Mach 12 vehicle as a function of Mach number.

lift and drag forces, but still predicts the general trend in forces. Several factors lead to these large errors: 1) violation of small disturbance assumptions near the maximum thickness location on the vehicle at positive angles of attack, 2) the assumption of no cross-flow (errors are significant on the upper surface at positive angles of attack), 3) small errors in pressure distribution are magnified when the vehicle has small net forces.

Comparing Figs. 7.1 and 7.9, the lift at supersonic Mach numbers is found to be almost an ordor of magnitude less than at high hypersonic speeds. Thus, at lower speeds, the airframe will most likely not be able to support its weight through aerodynamic lift. Some other means (such as engine thrust or wings) must be used to generate significant lift to keep the vehicle aloft. This is especially true during launch ascent. Therefore, cven though the model has relatively 


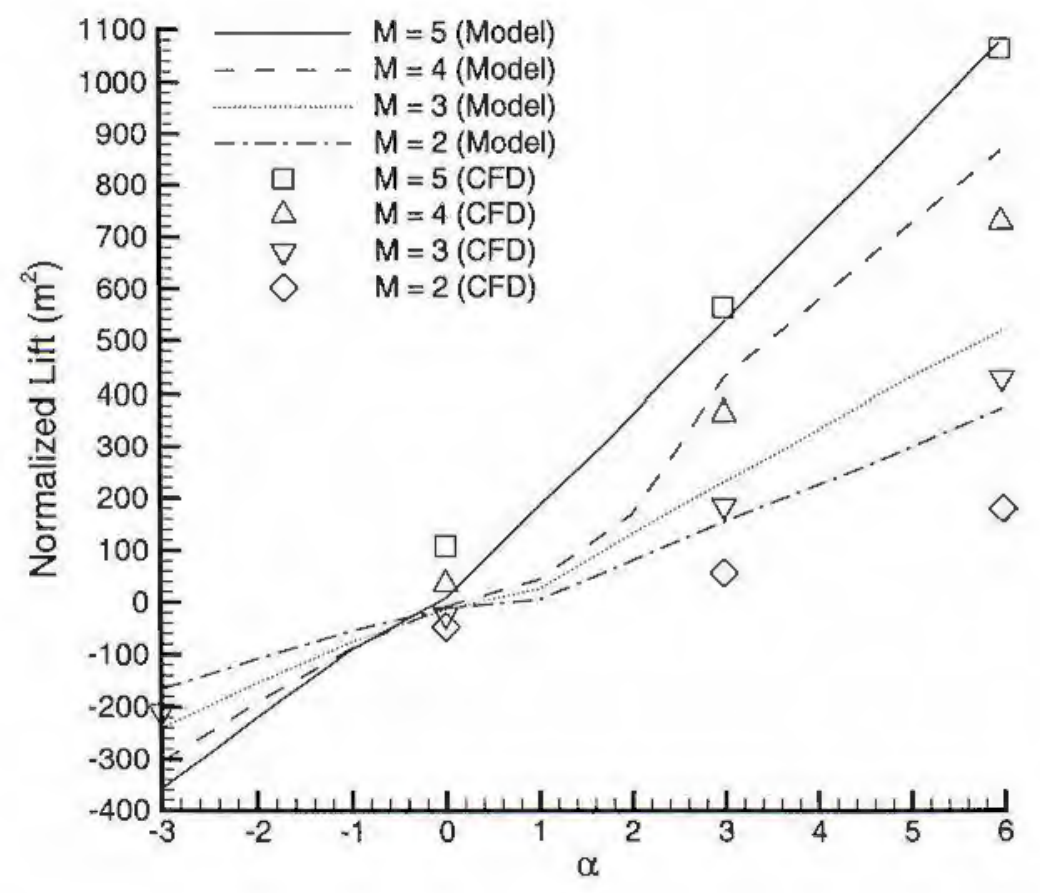

Figure 7.10: Supersonic lift $\left(\mathrm{m}^{2}\right)$ of Mach 12 vehicle as a function of angle of attack.

large errors in force prediction, it does predict that the vehicle will have low lift and also predicts the qualitative trend in lift. This is very useful in estimating when the fuselage will be able to support its own weight. Thus, the model is maintained for force prediction under the knowledge that the magnitudes of the forces are inaccurate, but the general trend towards zero lift is captured.

The normalized, inviscid, supersonic lift of the geometry is plotted as a function of angle of attack in Fig. 7.10 along with the computational results. The supersonic model captures the trends observed in Fig. 7.10 for Mach numbers above 2. The limits imposed by Eq. 6.5 again are apparent near zero angle of attack. A linear lift-curve slope is seen for the CFD solutions for all Mach numbers solved. The models capture the linear behavior for angles of attack greater 


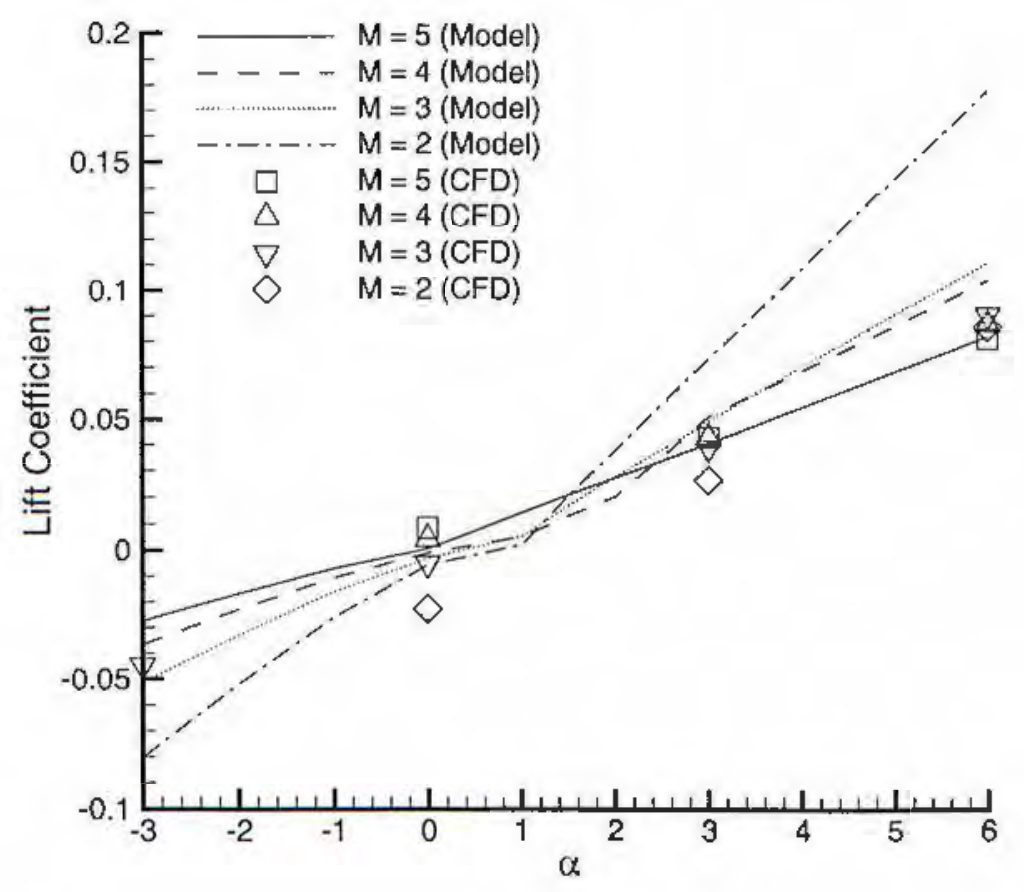

Figure 7.11: Supersonic lift coefficient of Mach 12 vehicle as a function of angle of attack.

than $3^{\circ}$.

The supersonic lift coefficient of the geometry is plotted as a function of angle of attack in Fig. 7.11 along with the computational results. The CFD results are observed to exhibit a linear trend in lift coefficient with respect to angle of attack. The supersonic model has linear portions away from near zero angle of attack. The model was found to roughly capture the trends for Mach number greater than 2, but clearly shows large errors in lift coefhicient prediction. At angle of attack near $6^{\circ}$, the CFD solutions show that the lift coefficient is roughly constant for a range of Mach number between 2 and 5.

The pressure drag at low Mach numbers, however, is more important to accurately predict. The normalized, inviscid drag of the geometry is plotted in 


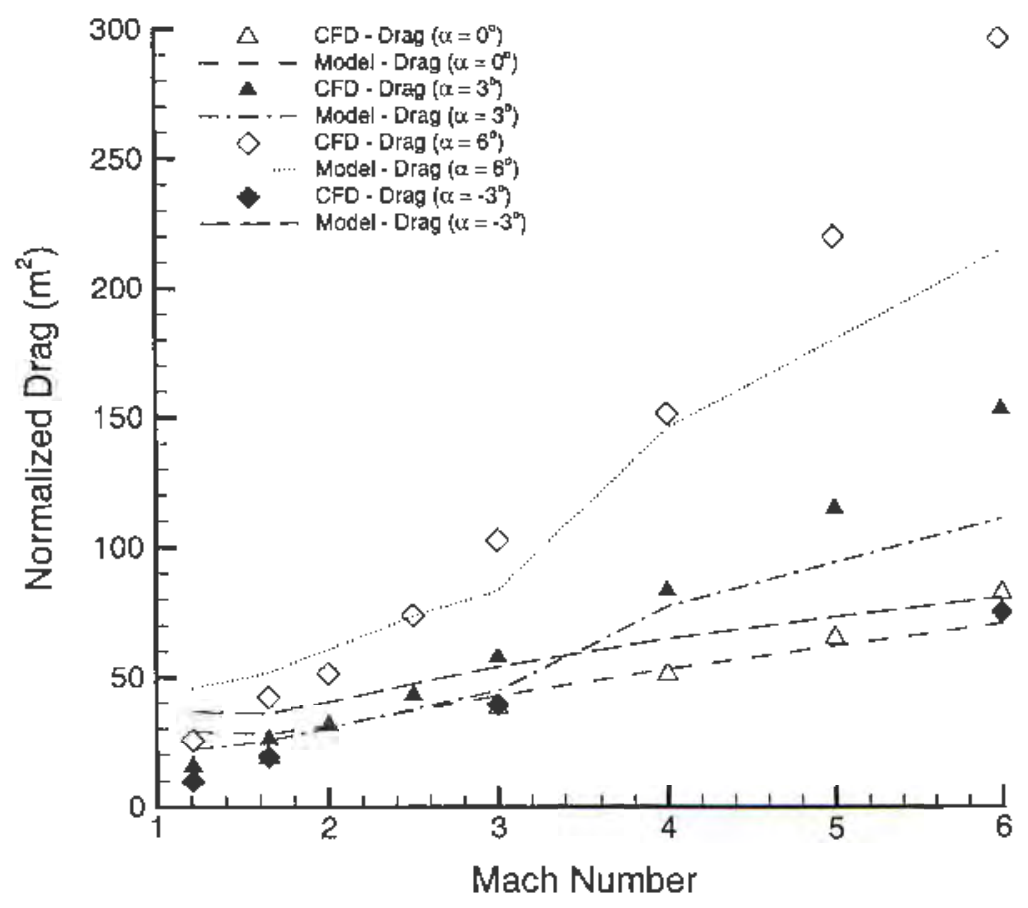

Figure 7.12: Supersonic, inviscid $\mathrm{drag}\left(\mathrm{m}^{2}\right)$ of Mach 12 velicle as a function of Mach number.

Fig. 7.12 along with the computational results. In general, the errors in drag are lower than the errors in lift, but are still unacceptable (above $\pm 30 \%$ ) for Mach numbers near sonic. The effect of the limit imposed by Eq. 6.5 is seen for the Mach 4 and 5 solutions near the zero angle of attack location. The drag is seen to increase with botlı Mach number and positive angle of attack.

The normalized, inviscid, supersonic drag of the geometry as a function of angle of attack is plotted in Fig. 7.13 along with the conputational results. The drag is observed to decrease with decreasing Mach number. A minimum in drag is observed for angle of attack near zero. The drag results tend to capture the behavior of the velicle as a function of angle of attack better than the lift results.

The supersonic, inviscid dagg coeficient of the geometry is plotted as a func- 


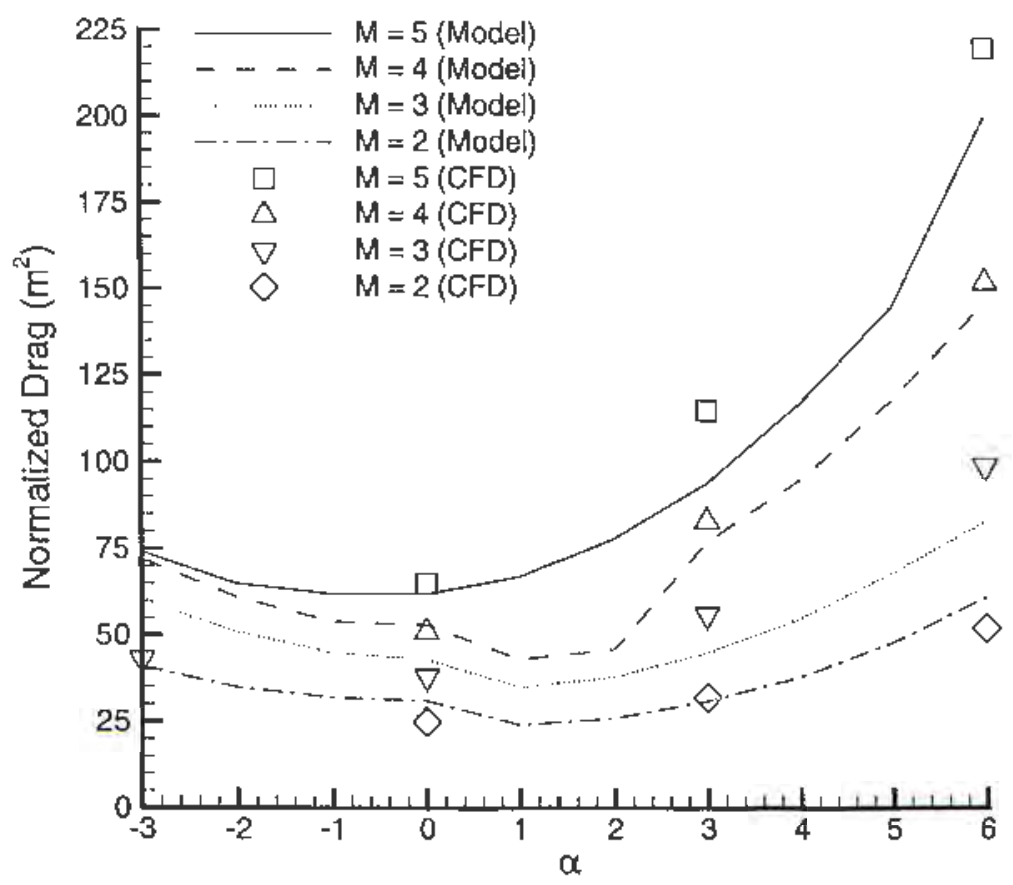

Figure 7.13: Supersonic, inviscid drag $\left(\mathrm{m}^{2}\right)$ of Mach 12 vehicle as a function of angle of attack.

tion of angle of attack in Fig. 7.14 along with the computational results. Drag coefficient is observed to increase with decreasing Mach number for both the CFD and model results. The limits imposed by Eq. 6.5 affects the solution for drag coefficient near zero angle of attack. Although the model does not quantitatively predict the trends in drag coefficient, it does capture the qualitative trends of the drag coefficient and predicts a minimutm drag coefficient.

The supersonic, inviscid drag polar is plotted in Fig. 7.15 along with the computational results. The drag coefficient was seen to have a minimum with respect to the lift coefficient. Both the CFD results and the supersonic model predict this trend, however the two methods differ for the quantitative trends. Overall, the supersonic model was found to give rough estinates for the lift 


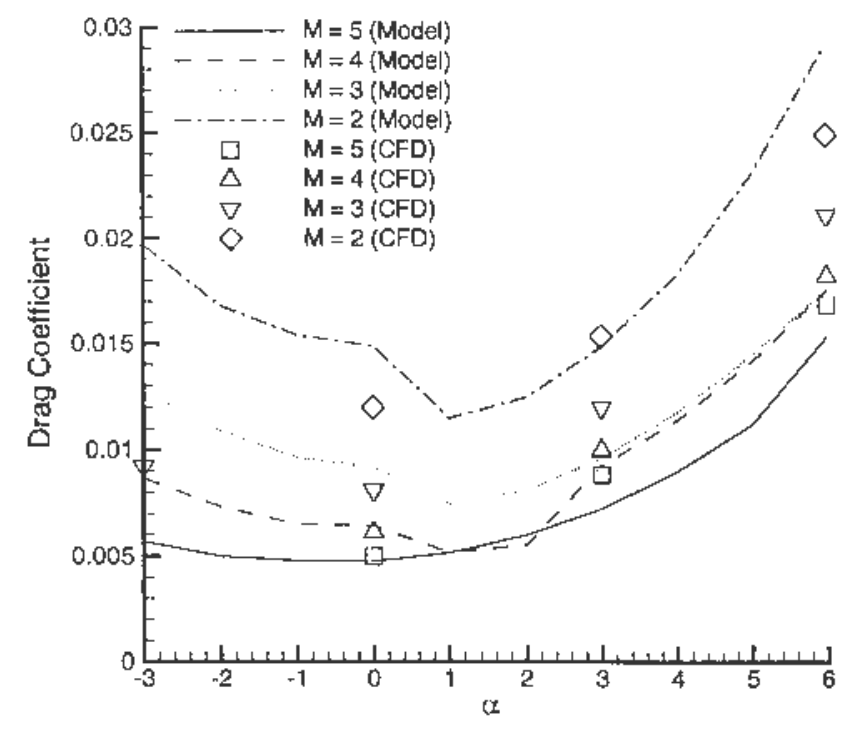

Figure 7.14: Supersonic, inviscid drag coefficient of Mach 12 vehicle as a function of angle of attack.

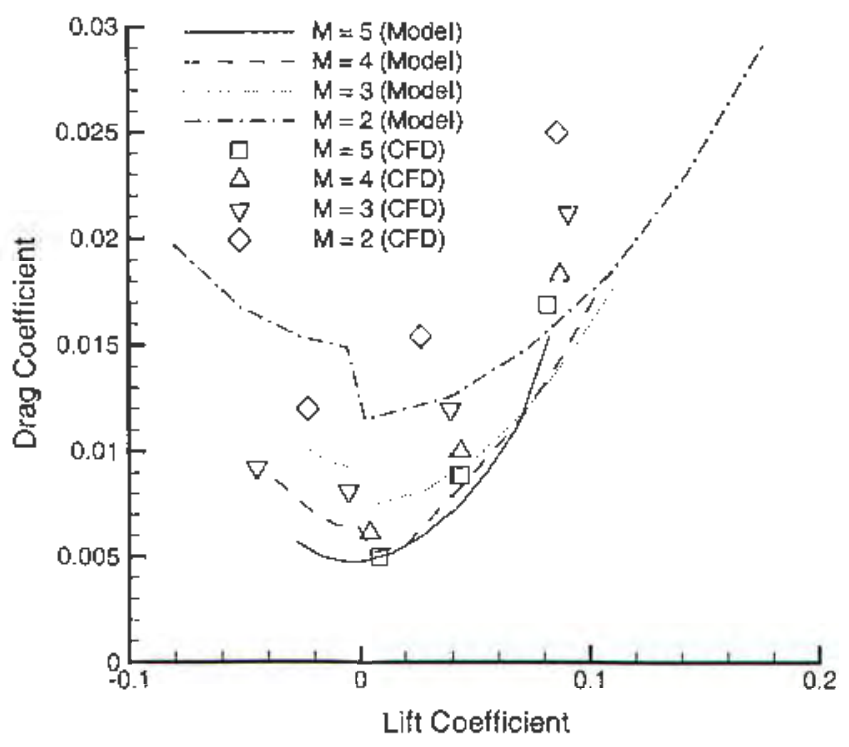

Figure 7.15: Supersonic, inviscid drag polar of Mach 12 vehicle. 
and drag trends of the fuselage. Improvements will be added to the model in Section 7.1.4.

\subsubsection{Transonic Performance}

A series of computational solutions were performed on the Mach 12 vehicle geometry presented in Chapter 4 for a range of Mach numbers between 0.4 and 5 and a range of angles of attack between $-3^{\circ}$ and $6^{\circ}$. The computational grid used for all the calculations is the same grid presented in Figs. 4.28 and 4.29. OVERFLOW ${ }^{74}$ is used to solve the 3-D, inviscid flowheld over the fuselage. The computational results are then compared to the transonic off-design models presented in Chapter 4.

The normalized, inviscid lift of the geometry is plotted in Fig. 7.16 along with the computational results. The lift was found to rise with increasing Mach number. The most immediately noticable feature of Fig. 7.16 is the large error in lift prediction. Taking the sonic solution at zero angle of attack as an example, the net lift on the vehicle is less than $1 \%$ of the lift force on either the lower or upper surface (see Tables 4.1 and 4.2). Hence, in order to accurately predict the lift at sonic Mach number, the prediction tool must be able to predict the lift on the lower and upper surface to less than $1 \%$ error. For the model developed in Section 4 , the error in lift on the lower surface was $-6.7 \%$ and $4.7 \%$ for the twodimensional and axisymmetric solutions, respectively. For the upper surface, the error in lift was $1.4 \%$. Thus, even though the model accurately predicts the lift forces on the lower and upper surfaces separately, combining the results yields an error in net lift of $934.5 \%$ and $-378.5 \%$ for the two-dimensional and axisymmetric solutions, respectively. As discussed above, at these low speeds, the fuselage can not be expected to provide any substantial lift for the vehicle. Thus, even though the model is poor at predicting the quantitative value of lift, 


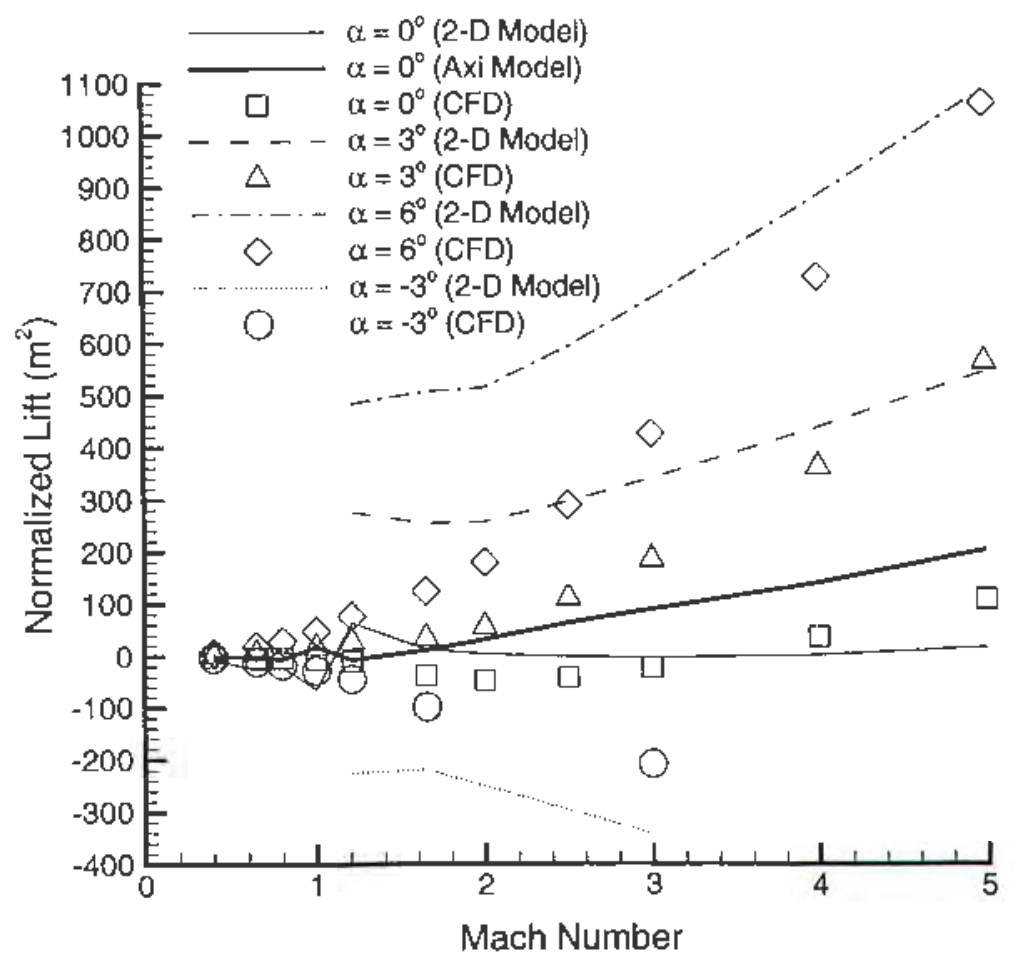

Figure 7.16: Transonic lift $\left(\mathrm{m}^{2}\right)$ of Mach 12 vehicle as a function of Mach number.

it does predict the qualitative trend of very little lift at transonic conditions.

The normalized, inviscid, transonic lift of the geonnetry is plotted as a function of angle of attack in Fig. 7.17 along with the computational results. Both the computational solutions and the transonic models predict a linear trend in lift as a function of angle of attack. However, for Mach numbers greater than one the lift was overpredicted compared to the computational results. The lift errors are a result of overpredicting the forcbody compression for the two-dimensional resilts.

The transonic lift coefficient of the geometry is plotted as a function of angle of attack in Fig. 7.18 along with the computational results. The computational results display a linear slope for the lift coefficient as a function of angle of attack. The transonic model displays the sane tendency, but has large errors (especially 


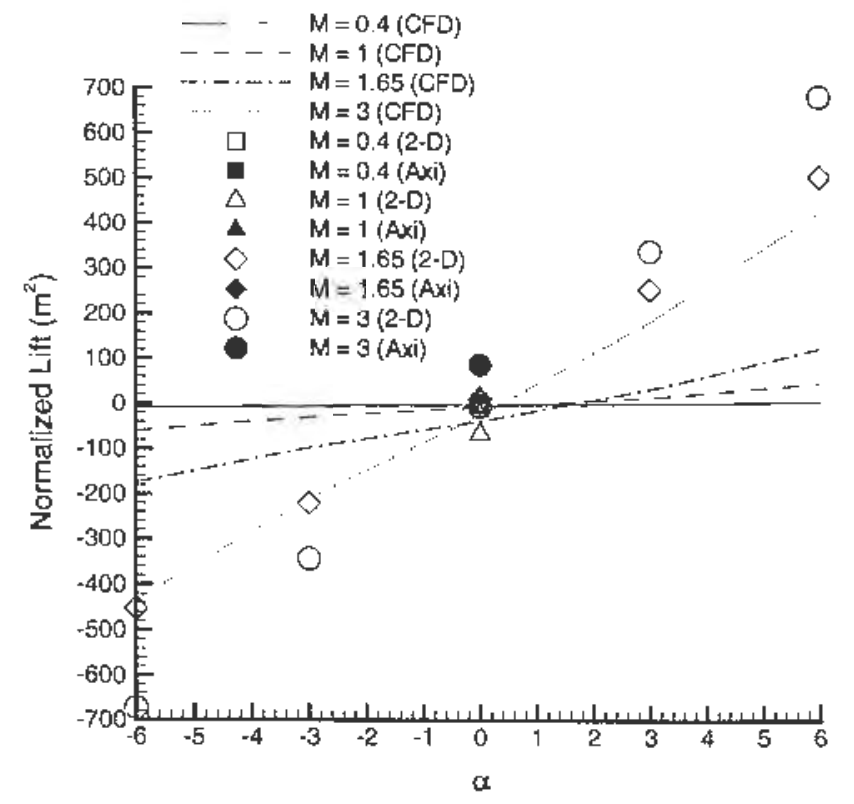

Figure 7.17: Transonic lift $\left(\mathrm{m}^{2}\right)$ of Mach 12 vehicle as a function of angle of attack.

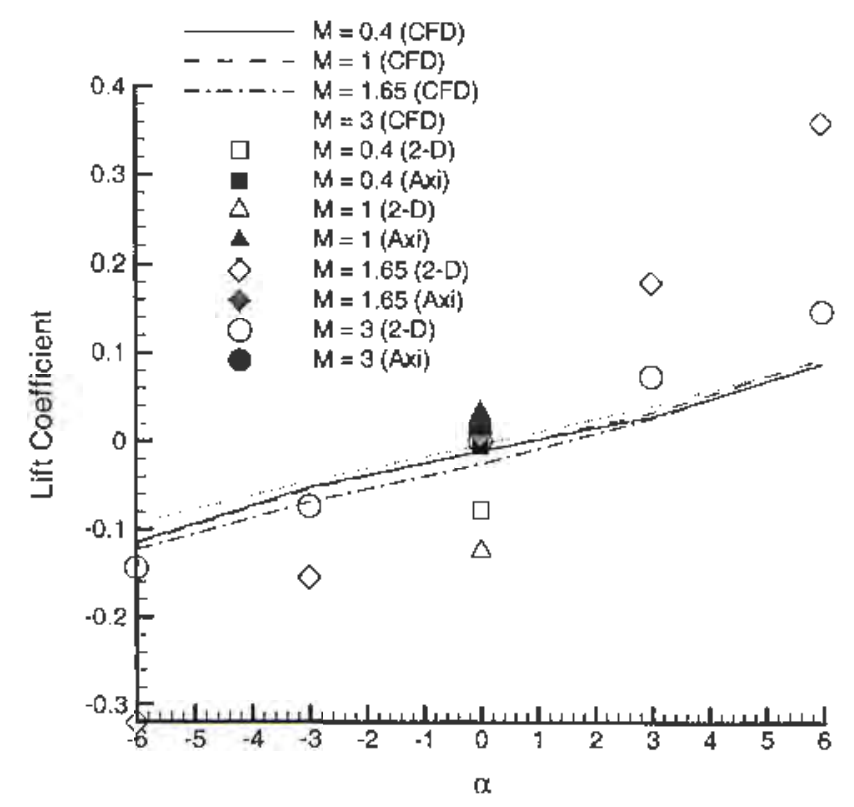

Figure 7.18: Transonic lift coefficient of Mach 12 vehicle as a function of angle of attack. 


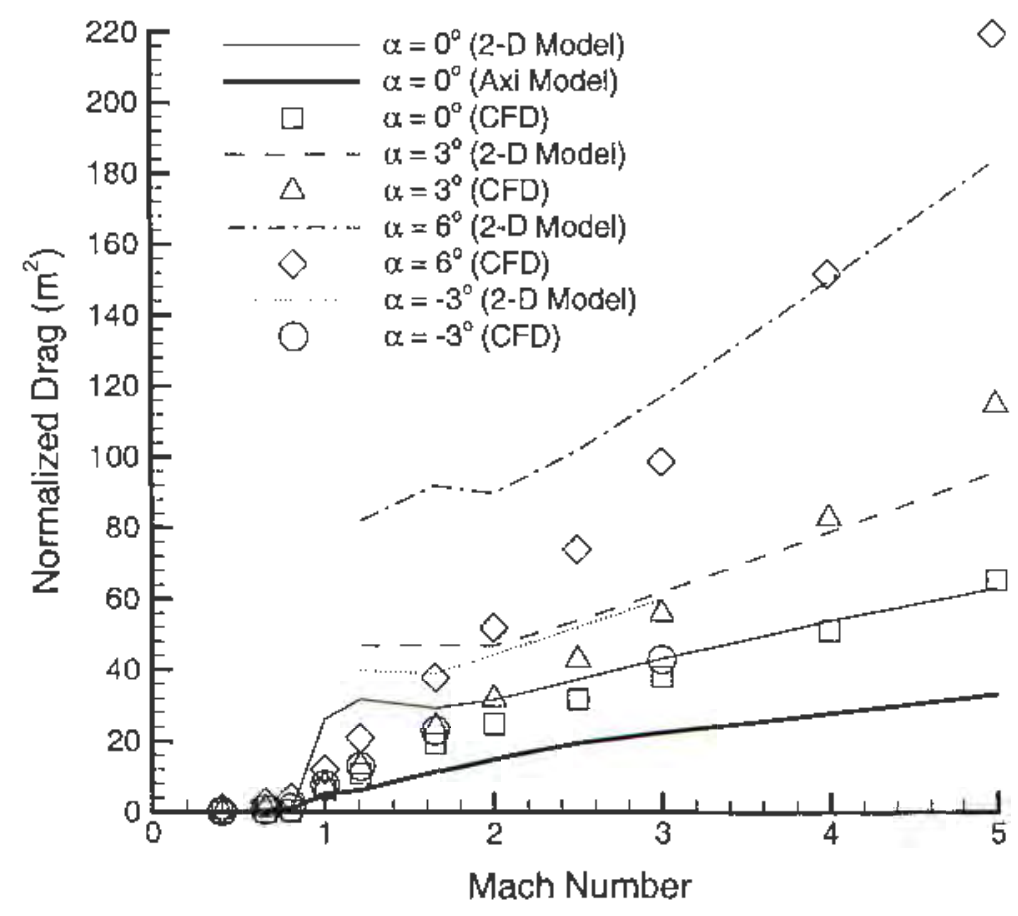

Figure 7.19: Transonic inviscid drag $\left(\mathrm{m}^{2}\right)$ of Mach 12 vehiclc as a function of Mach number.

for higl Mach number). The axisymmetric results at zero angle of attack tend to yield the best predictions for the lift coefficient.

The pressure drag predictions shown in Fig. 7.19 demonstrate much better behavior than the lift predictions. The two-dimensional solution at zero angle of attack was found to model the pressure drag at higher Mach numhers quite well, with errors between $27.8 \%$ and $-3.5 \%$ for Mach numbers betwen 2 and 5. Also, the pressure drag is predicted to be essentially zero for the subsonic solutions (expected from d'Alembert's paradox ${ }^{78}$ ). The largest error for the two-dimensional solutions was found to be in the sonic area, with crrors on the order of $200 \%$. In contrast, the axisymmetric solutions behave well at lower speeds, only deviating substantially for Mach numbers greater than 1.5. Hence, 


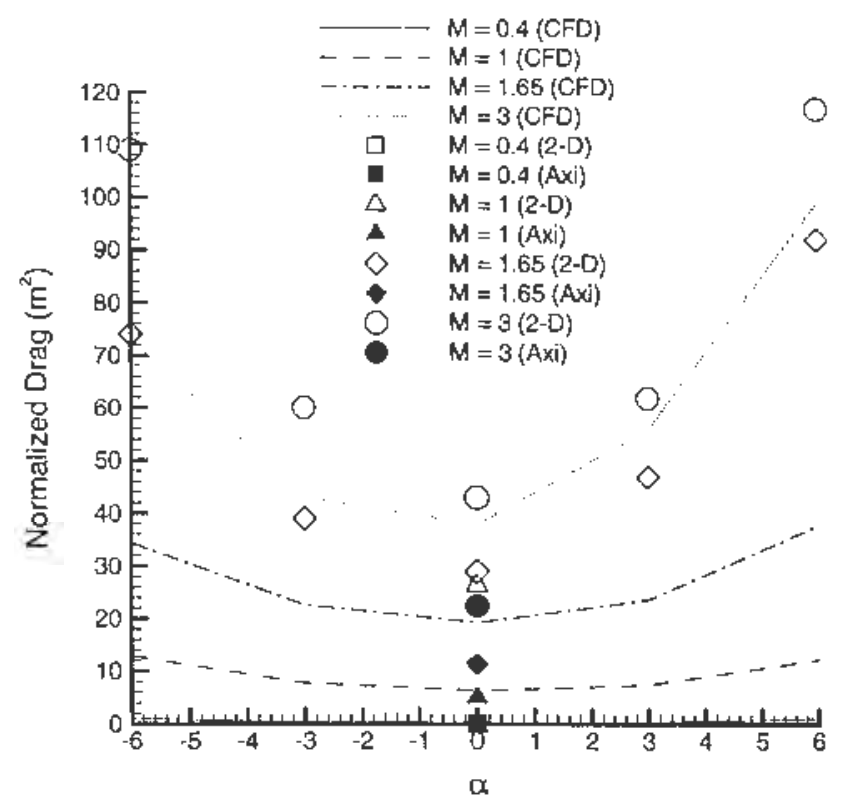

Figure 7.20: Transonic, inviscid drag $\left(\mathrm{m}^{2}\right)$ of Mach 12 vehicle as a function of angle of attack.

by combining the two solutions together, an accurate means of predicting the pressure drag on the geometry is found between the Mach numbers of 0.4 and 5. In the following section, more details will be given on how all of the methods discussed above are combined together to predict the forces on the vehicle.

The normalized, inviseid, transonic drag of the geometry is plotted as a function of angle of attack in Fig. 7.20 along with the computational results. The computational results show a minimum in drag ncar zero angle of attack, with increasing drag for both positive and negative angle of attack. The transonic model has the sane tendency, but overpredicts the drag (a result of overpredicting the forebody compression).

The transonic, inviscid drag coefficient of the geometry is plotted as a function of angle of attack in Fig. 7.21 along with the computational results. The computational results display the same behavior found in Fig. 7.20. The CFD 


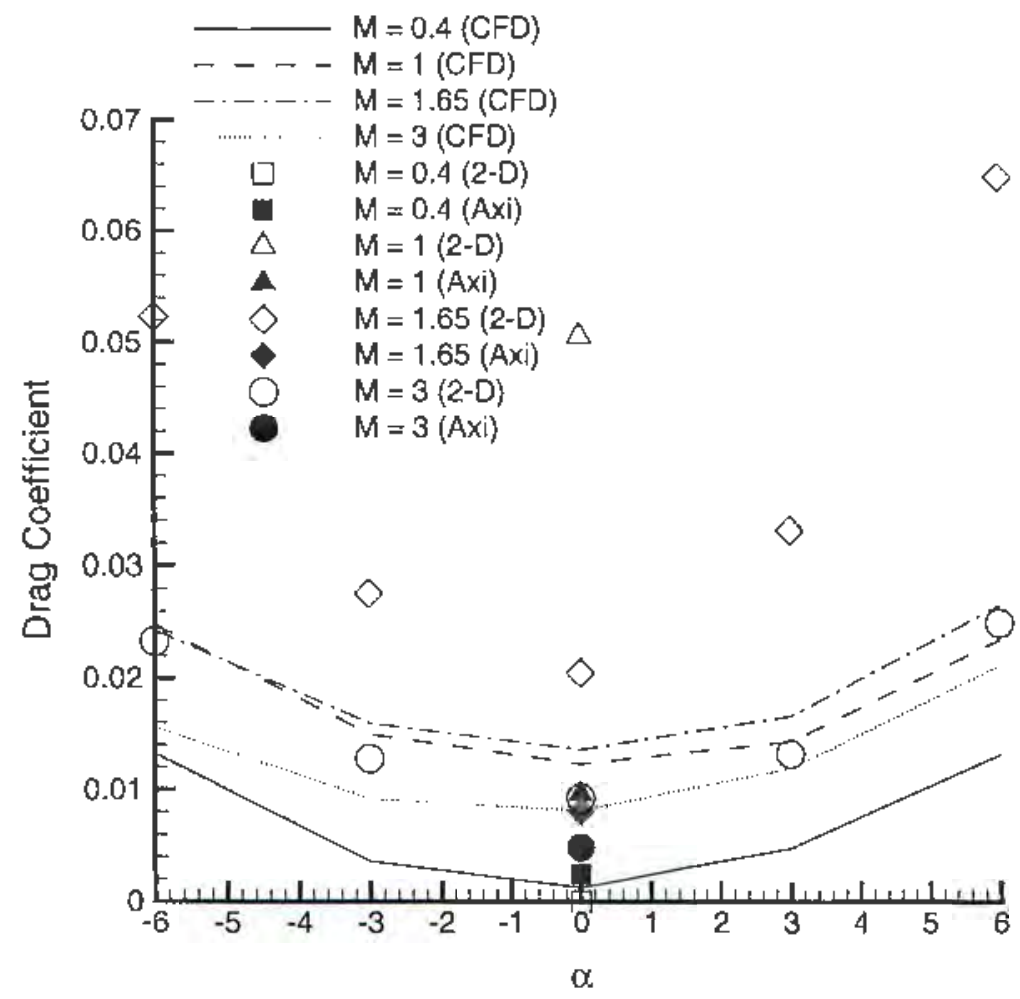

Figure 7.21: Transonic, inviscid drag coefficient of Mach 12 vehicle as a function of angle of attack.

calculations showed that the inviscid drag coefficient increases from Mach 0.4 to Mach 1.65, then decreases for Mach 3. Hence, the computational results conclude that the maximum inviscid drag coefficient occurs somewhere above Mach 1. In contrast, for the viscous drag coefficient, it would be expected that the maximum drag coefficient would occur at Mach 1. The transonic models tend to predict the drag coofficient at zero angle of attack better than at angle of attack. They also tend to predict the drag coefficient better at low Mach number than high Mach number.

The transonic, inviscid drag polar is plotted in Fig. 7.22 along with the computational results. The CFD results showed that the minimum drag coeficient occurred near zero-lift. This is in contrast to the supersonic and hypersonic 


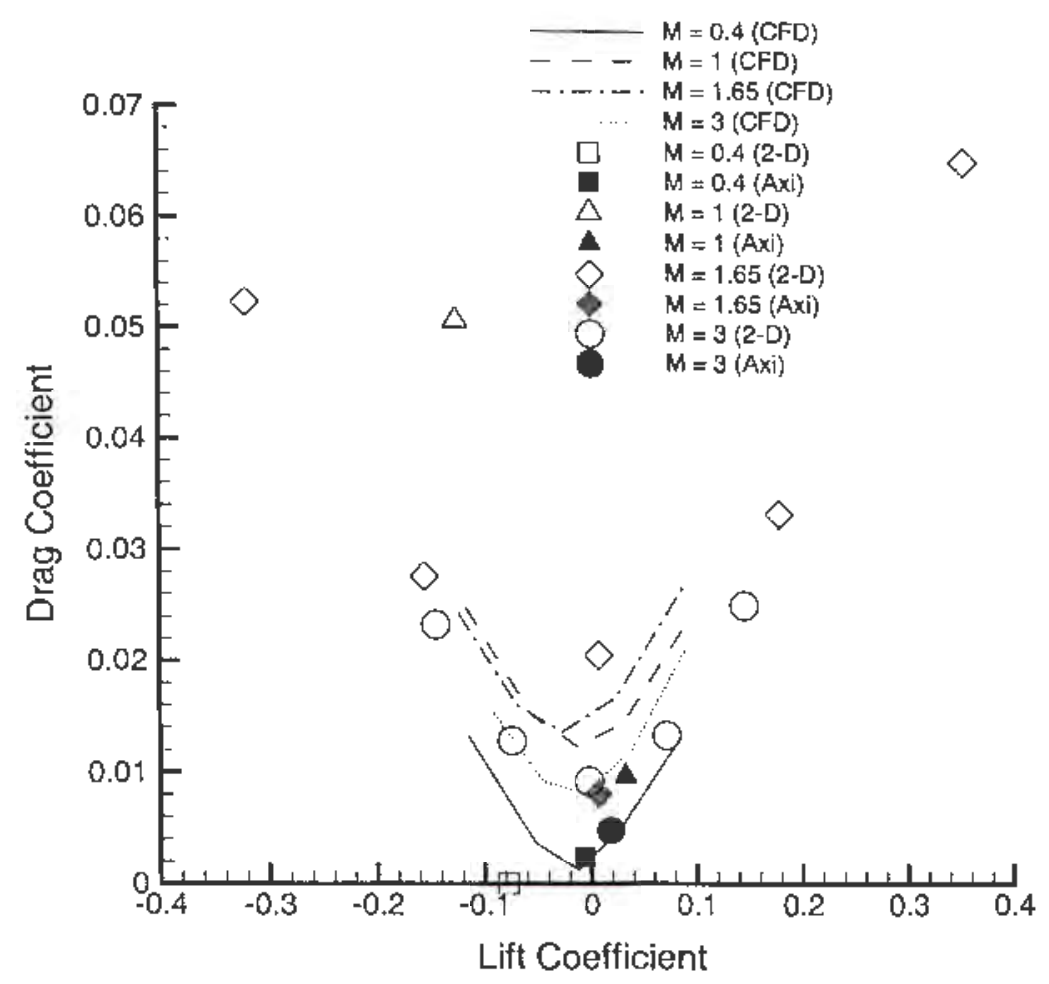

Figure 7.22: Transonic, inviscid drag polar of Mach 12 vehicle.

rcsults, which showed that minimum drag coefficient occurred for negative lift coefficiont (negative angle of attack). The transonic models tended to predict the rlrag polars better at lower Mach number and low lift coefficients.

\subsubsection{Blending of Methods}

In developing the above models, certain aspects of each solution lend themselves toward an overall better solution for the vohicle. Thus, several methods may be used in concert to predict not lift and pressure drag better than any individial model. Table 7.2 shows which methods work for a range of Mach numbers and angles of attack. The notation is identified as: T2D - transonic: two-dinensional solution, Taxi - transonic axisymmetric solution, S2D - super- 


\begin{tabular}{ccc}
\hline \hline$M_{\infty}$ & $\alpha$ & Method \\
\hline 0.4 & $=0$ & T2D \\
\hline 0.65 & $=0$ & T2D: top, Taxi: bot \\
\hline & $>0$ & T2D: top, Saxi: bot \\
1.21 & $=0$ & S2D: top, $L_{b} ;$ T2D: $T_{b} ;$ Taxi: $D_{b}$ \\
& $<0$ & Saxi: top, T2D: bot \\
\hline & $>0$ & H: top; Saxi: $L_{b}, T_{b} ;$ S2D: $D_{b}$ \\
3 & $=0$ & S2D \\
& $<0$ & Saxi: top, T2D: bot \\
\hline & $>0$ & H: top, S2D: bot \\
4 & $=0$ & S2D \\
& $<0$ & Saxi: top, T2D: bot \\
\hline & $>0$ & H \\
5 & $=0$ & S2D: top, H: bot \\
& $<0$ & Saxi: top, T2D: bot \\
\hline 6 & $\geq 0$ & H \\
\hline \hline & $<0$ & S2D: top, H: bot \\
\hline
\end{tabular}

Table 7.2: Methods used in solving for aerodynamic forces. 
sonic two-dimensional solution, Saxi - supersonic axisymmetric solution, $L_{b}$ - lift force on the bottom surface, $T_{b}$ - thrust force on the bottom surface, $D_{b}$ - drag force on the bottom surface, $\mathrm{H}$ - hypersonic solution. To determine which solution to use, the method listed in each row may be applied from the Mach number listed to the following Mach number. For example, between Mach 0.4 and Mach 0.65 , the two-dimensional transonic solution is used for the entire vehicle at zero angle of attack.

Some general trends are noticed in Table 7.2. First, the hypersonic method works well for all angles of attack down to Mach 7. Below Mach 7, the hypersonic solution is phased out while the supersonic solution phases in. By Mach 3, the hypersonic solution is completely phased out in favor of supersonic methods. At Mach 3, the supersonic solutions are beginning to phase out while the transonic solutions are beginning to phase in. By Mach 1, the supersonic solutions are phased out completely in favor of the transonic methods. These methods are then applied all the way down to incompressible flow. By this methodology, the flowfield on these geometries may now be completely solved for a wide range of Mach numbers and angles of attack. The methodology discussed in the previous sections will be applied according to Table 7.2 to provide overall results.

Using the approach outlined in Table 7.2, the net lift on the example Mach 12 vehicle is compared to the computational results in Fig. 7.23 for a range of Mach numbers between 0.4 and 15 and for angles of attack between $-3^{\circ}$ and $6^{\circ}$. The models were found to give excellent predictions of the lift trends as a function of both Mach number and angle of attack. For Mach numbers below 4 , the magnitude of lift (even at $6^{\circ}$ angle of attack) was low compared to the hypersonic regime. As the magnitude of lift decreases, the percent error in the prediction of lift increases, shown in Fig. 7.24 as a function of Mach number and angle of attack. However, in general, the error in the magnitude of the lift error, shown in Fig. 7.25 is observed to decrease with decreasing Mach number. Thus, 


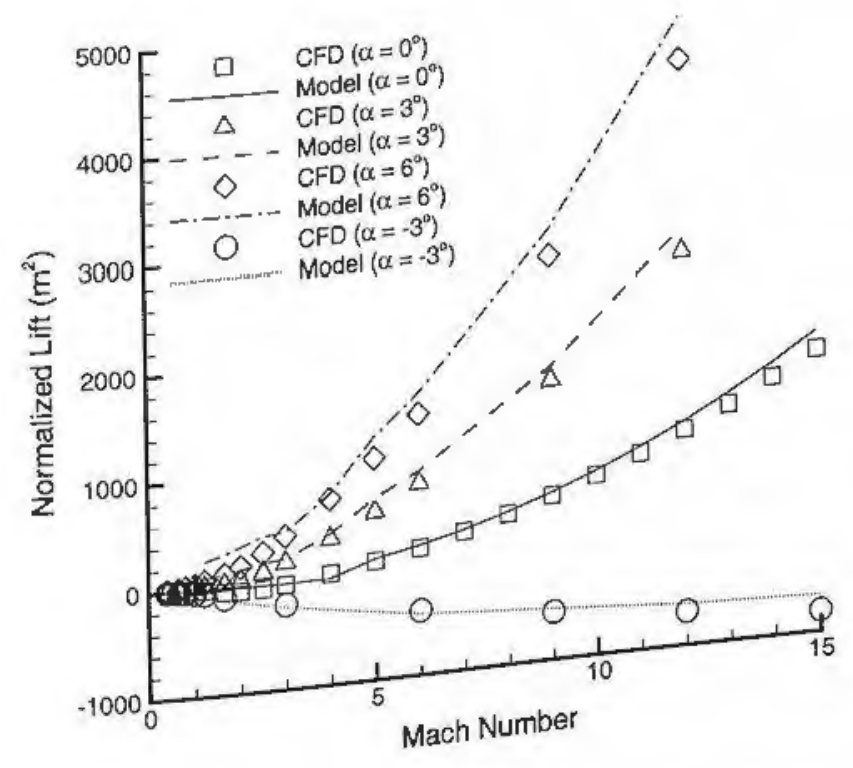

Figure 7.23: Lift on example Mach 12 vehicle for full Mach number range.

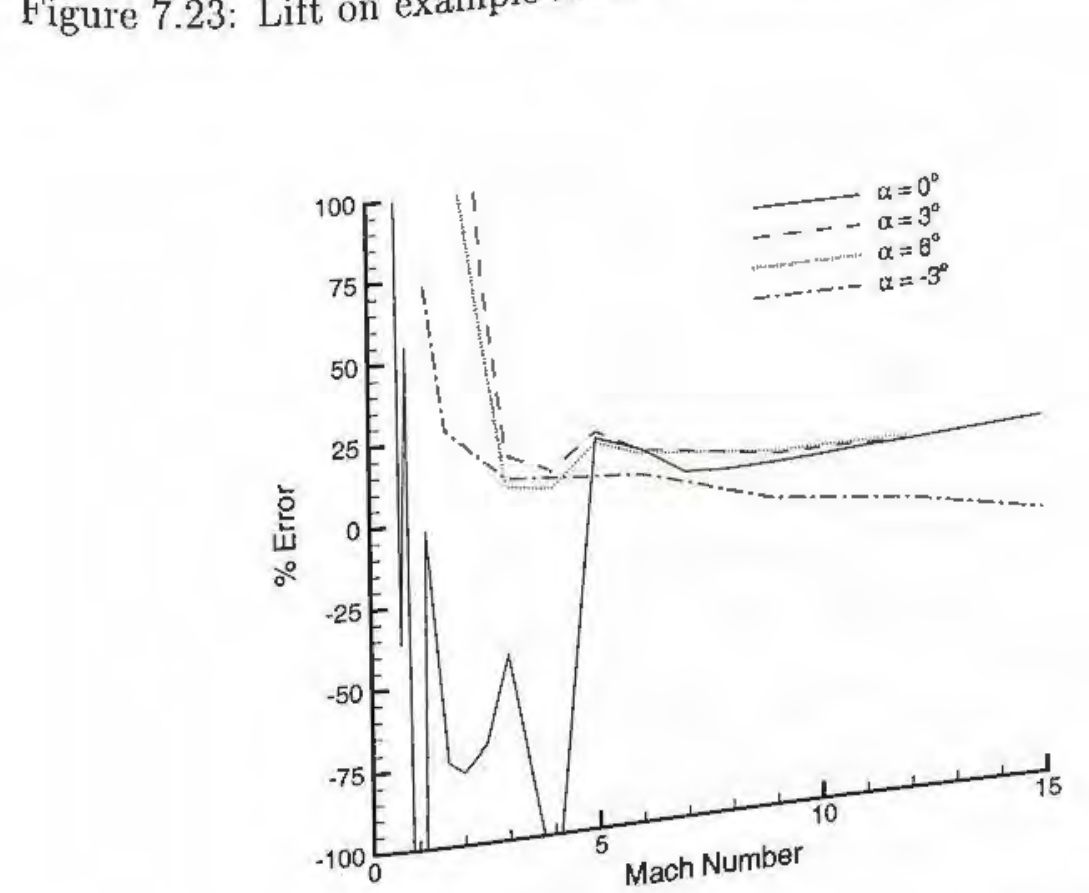

Figure 7.24: Percent error in lift on example Mach 12 vehicle for full Mach number range. 


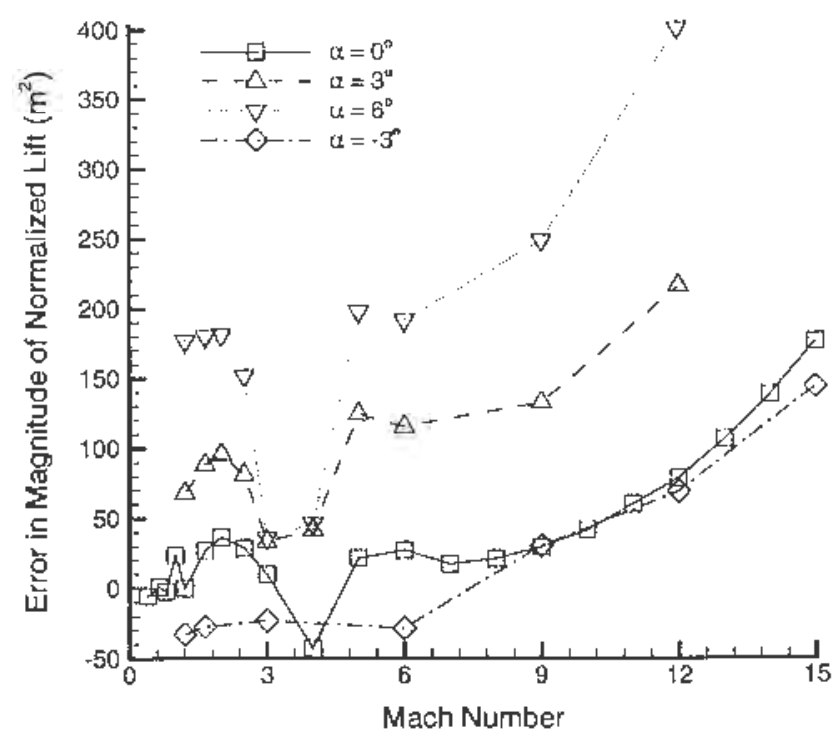

Figure 7.25: Error in magnitude of lift on example Mach 12 vehicle for full Mach number rangc.

even though the error in lift prodiction using the analytical methods is large at low speeds, the general trend in lift as a function of both Mach number and angle of attack is captured quite well.

The lift coeficient of the example Mach 12 vehicle is plotted as a function of Mach number in Fig. 7.26. The methodology outlined in Tabie 7.2 gives excellent agroment with the computational rosults for Mach numbers greater than or equal to three. Below Mach 3, the results from Table 7.2 for angle of attack are socn to diverge as Mach number goes to 1. For zero angle of attack, tho lift coefficiont stays relatively constant from Mach 15 down to Mach 4. Below Mach 4, the lift coefficient goes negative and stays there down to the lowest solution of Mach 0.4 . For angle of attack of $3^{\circ}$, the lift coefficient stays relatively constant down to Mach 6 . The lift coefficiont is then found to increase slightly to a maximum at Mach 4 , followed by a decrease in lift coefficient with a minimum 


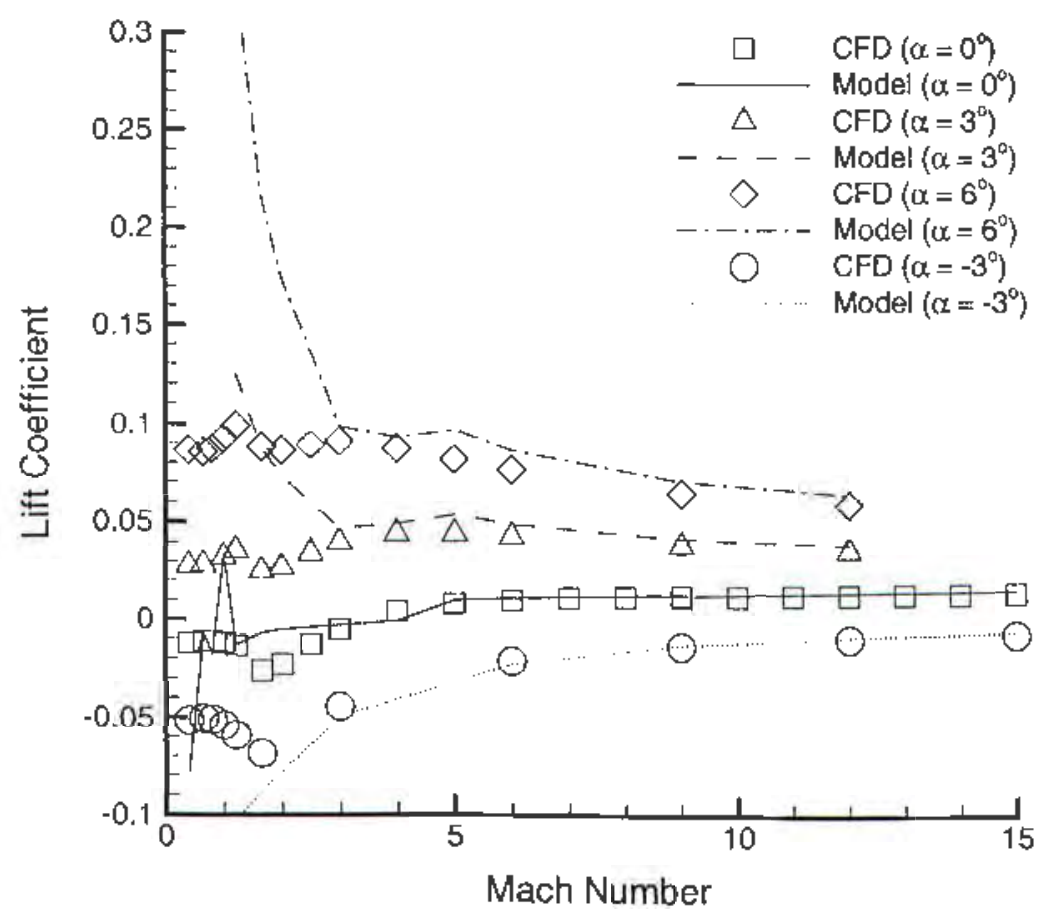

Figure 7.26: Lift coefficient on example Mach 12 vehicle for full Mach number rànge.

near Mach 1.65. For angle of attack of $6^{\circ}$, the lift coefficient is observed to increase with decreasing Mach number, reaching a maximum near Mach 1.2.

Several interesting notes are observed in Fig. 7.26. The first observation is that the lift coefficient for all angles of attack solved was rather invariant with respect to Mach number. This is quite a surprise, considering the very large variation of Mach number solved for this geometry. Another interesting note is the magnitude of maximum lift coefficient. The maximum $C_{l}$ found for this geonctry was approximatcly 0.1 for a Mach number of 1.21 and an angle of attack of $6^{\circ}$. To put this lift coefficient in perspective, the lift coefficient for the Wright number 12 airfoil design (the airfoil that infuenced their 1902 glider) at an angle of attack of $6^{\circ}$ was on the order of $0.6^{79}$. Thus, the wing for the 1902 


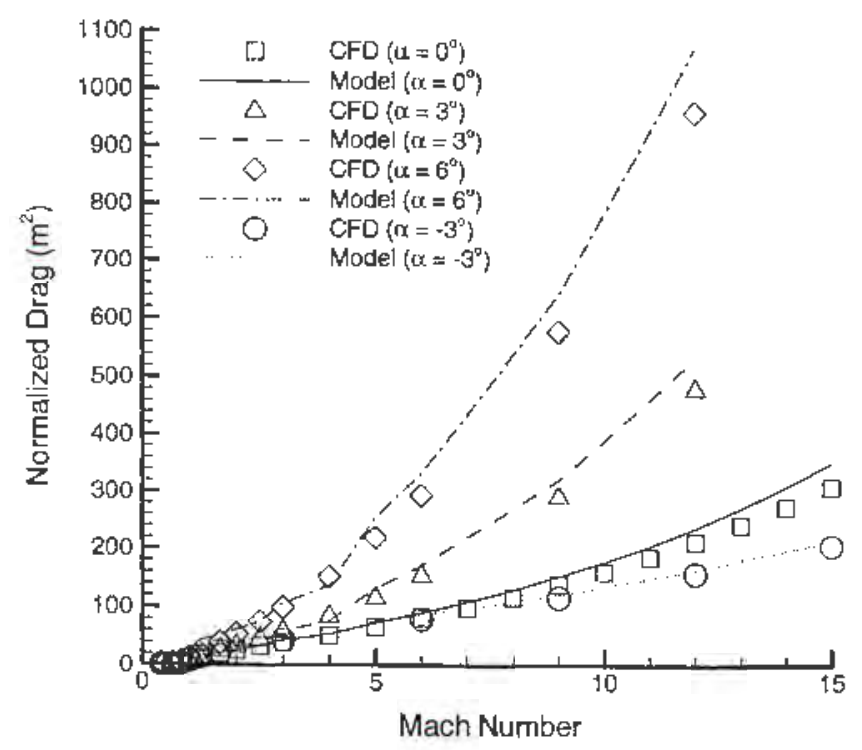

Figure 7.27: Pressure drag on example Mach 12 vehicle for full Mach number range.

Wright glider had a lift coefficicnt 6 times greater than the current geometry does at Mach 1.2! From these observations, it is clear that this geometry will not be able to produce a significant amount of lift at take-off conditions without large angle of attack, high take-off speeds, and a significant amount of lift generated directly by the engine. IIence, some other means of producing low-speed lift: such as wings and/or canards will most likely be necessary to achieve sufticient lift for take-off.

The pressure drag on the example Mach 12 vehicle (using the approach outliner in Table 7.2) is compared to the computational results in Fig. 7.27 for a range of Mach numbers between 0.4 and 15 and for angles of attack between $-3^{\circ}$ and $6^{\circ}$. The analytical methods accurately capture the trends in pressure drag for both Mach mumber and angle of attack. At lower speeds, the models are less accurate in the prediction of the magnitide of pressure drag. Olsserving 


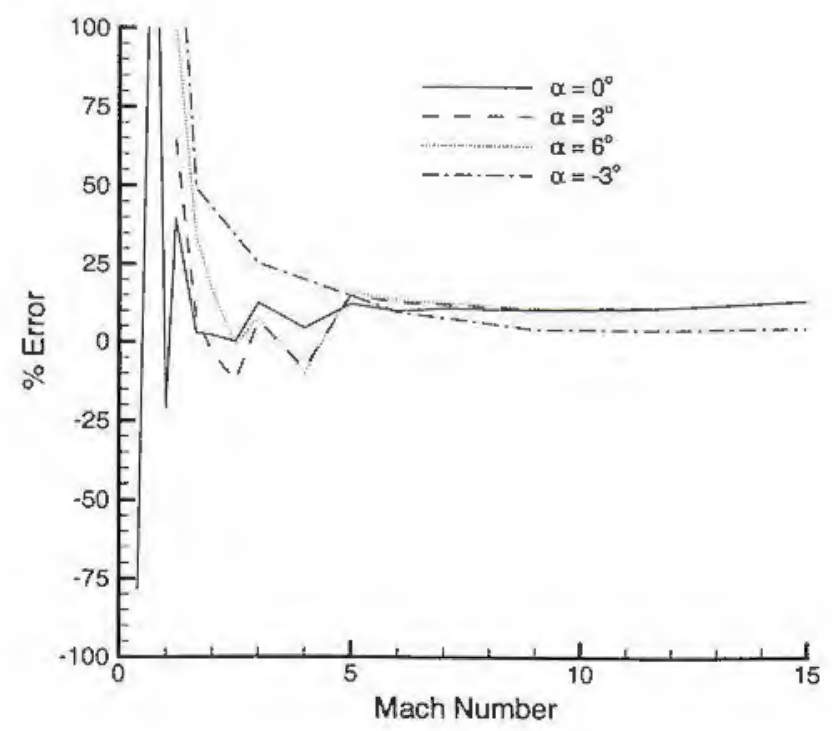

Figure 7.28: Percent error in pressure drag on example Mach 12 vehicle for full Mach number range.

Fig. 7.28, as the magnitude of pressure drag decreases, the error in pressure drag prediction increases. This behavior is similar to that displayed by the lift plots in Figs. 7.23 and 7.24. Therefore, even though the magnitudes of lift and pressure drag are erroneous at low speeds, the model captures the general trends for the forces on the vehicle for a wide range of Mach numbers and angles of attack.

Figure 7.29 shows the inviscid drag coefficient of the Mach 12 example vehicle plotted as a function of Mach number. The drag coefficient is observed to increase with positive angle of attack for all Mach numbers. For negative angles of attack, the drag coefficient may either increase or decrease as a function of angle of attack, depending on the freestream Mach number. The results of Table 7.2 tend to give good predictions of drag coefficient with angle of attack for Mach numbers greater than or equal to 2 (with the exception of negative angles of attack, where the predictions are good after Mach 6). The zero angle of attack results 


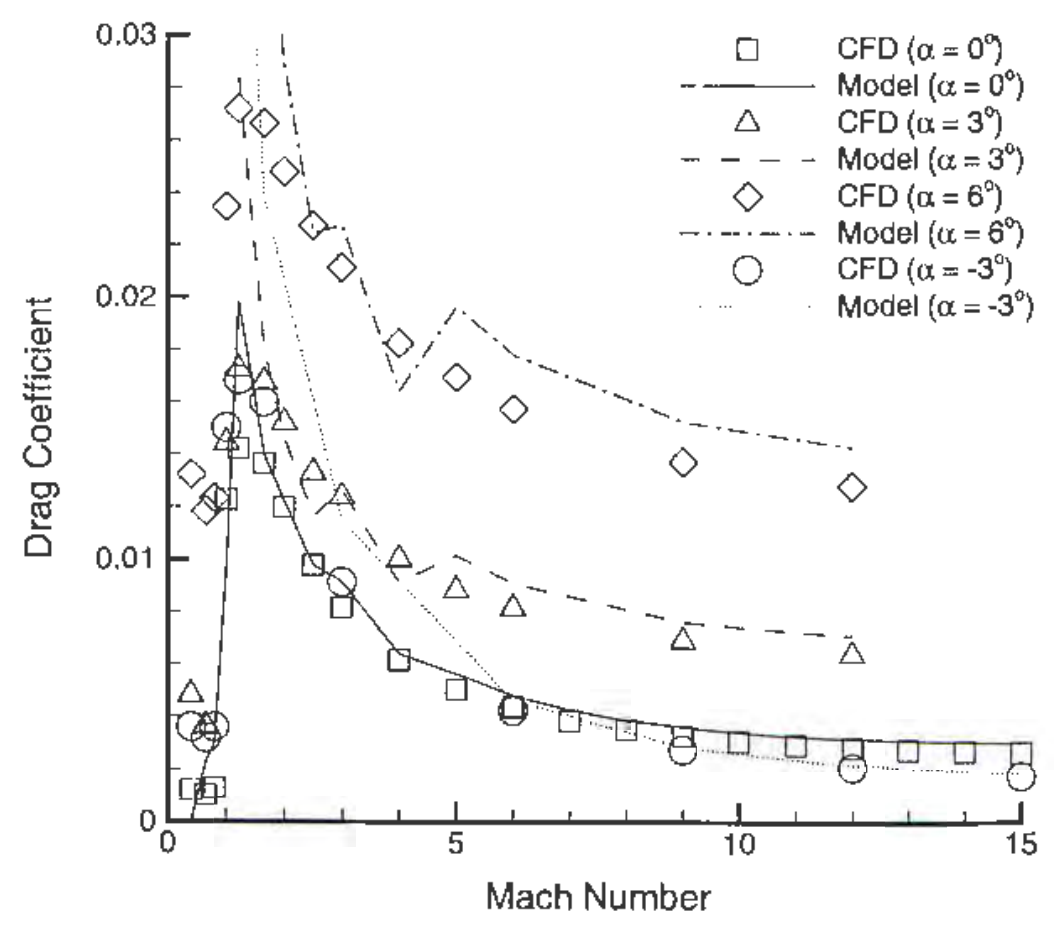

Figure 7.29: Inviscid drag cocfficient on example Mach 12 vehicle for full Mach nitumber range.

were found to give excellent predictions of the drag coefficient trend (including an accurate prediction of the Mach number corresponding to maximum drag coefficient) across the full Mach number range. However, the method outlined in Table 7.2 tends to overpredict the maximum drag coefficient by over $40 \%$.

The maximum inviscid drag coefficient for all angles of attack was observed in Fig. 7.29 to occur near Mach 1.2. This is in contrast to the general trend of maximum viscous drag coefiicient occuring at Mach 1. This behavior was observed for cvery angle of attack solved. Another interesting note is that while the lift coefficient was found to be substantially lower than typical low-speed lift coefficients, the inviscid drag coefficient was found to bo reasonable for a low-speed configuration. 


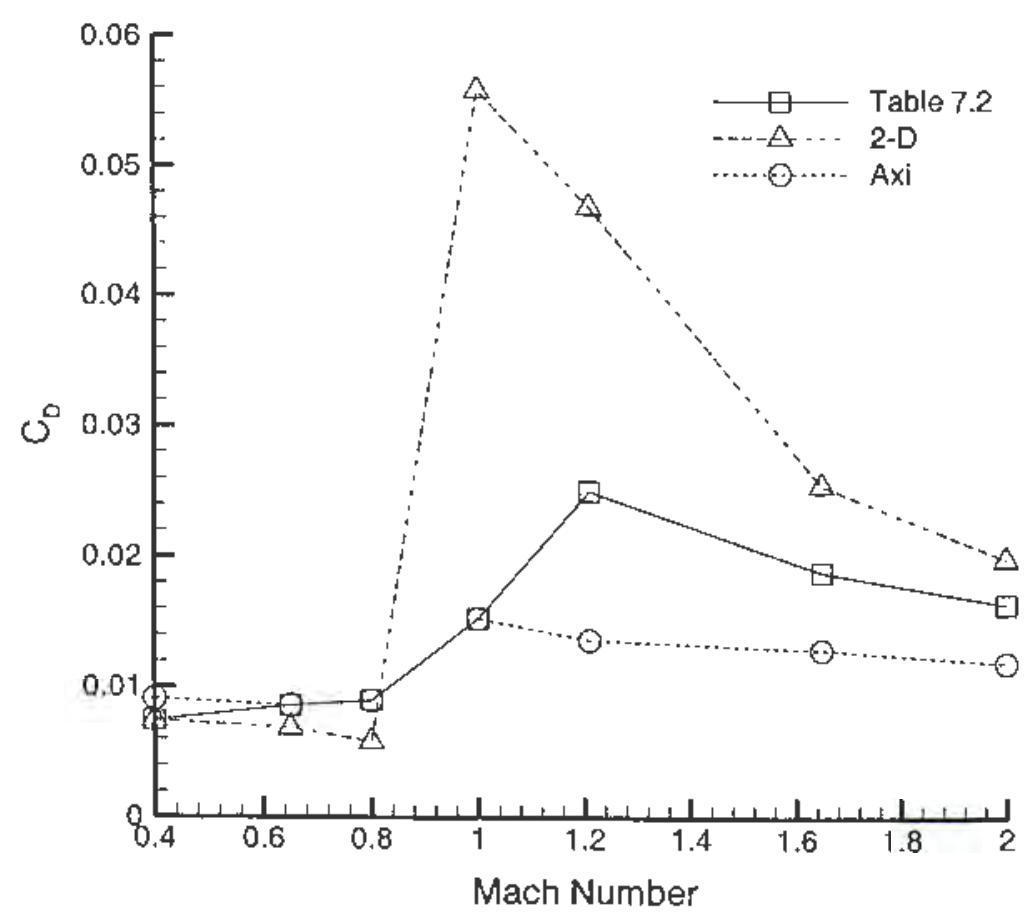

Figure 7.30: Transonic, viscous airframe drag coefficient as a function of Mach number.

An essential feature in modeling the transonic behavior of a vehicle is capturing the increase in drag coefficient (the transonic drag-rise or "barrier") in the neighborhood of Mach 1. The pressure distributions predicted with the methods outlined in Table 7.2 may be used in estimating the Mach number by assuming isentropic flow. With Mach number and pressure, the turbulent friction coefficient may be estimated using the methods discussed in Section 2.6 .2 (assuming a wall temperature of $300 \mathrm{~K}$ ). The total drag coefficient is plotted as a function of Mach number in Fig. 7.30 for zero degrees angle of attack. Along with the results using Table 7.2 are the drag coefficients calculated using the two-dimensional transonic method and the axisymmetric transonic method.

The drag coefficiont calculated using Table 7.2 was found to have a maximum 
value near Mach number of 1.21 (consistent with the inviscid results plotted in Fig. 7.29). For the transonic methods, the peak in drag coeflicient was calculated to be at Mach 1. The reason for this discrepancy is that the blending of solutions used in Table 7.2 switches between several different types of flows, and does not maintain one specific method in the sonic regime. Observing the wide difference in drag coefficient between the two-dimensional and axisymmetric methods in Fig. 7.30, it is not surprising that when the two methods are blended together the resulting drag coefficient does not exhibit the desired behavior.

Prior to sonic Mach number, the drag coefficient is seen to remain rather constant. This is consistent with the behavior of compressible flow prior to the drag divergence Mach number, where the primary source of drag comes from viscosity. Since the subsonic solutions for the transonic methods described above are for purcly subsonic flow, Macl numbers above subcritical aro still moxleled as behaving subsonically, cven though supersonic flow exists at some location on the geometry. 'Thus, the drag divergence bellavior is not exhibited. After Mach 1, the drag coefficient is secn to decrease (except for the Table 7.2 solution, where it decreases after Mach 1.21) monotonically in the same manner as expected for supersonic flow.

An interesting phenomenon is that the drag coefficient predicted using twodimensional flow is substantially higher than the drag coefficient using axisymmetric flow in the supersonic regime. This phenomenon is a result of the assumption of no cross-flow. By not modeling the three-dirnensional pressure relieving, the calculated pressures on the lower surface of the vehicle are higher than they should be. Highcr pressures translate into higher pressure drag as well as ligher viscous drag.

The inviscid and viscous lift-to-drag ratio are compared to the inviscid computational solution in Fig. 7.31 for a range of Mach numbers between 0.4 and 15. For the viscous calculations, the wall temperature was considered to be a 


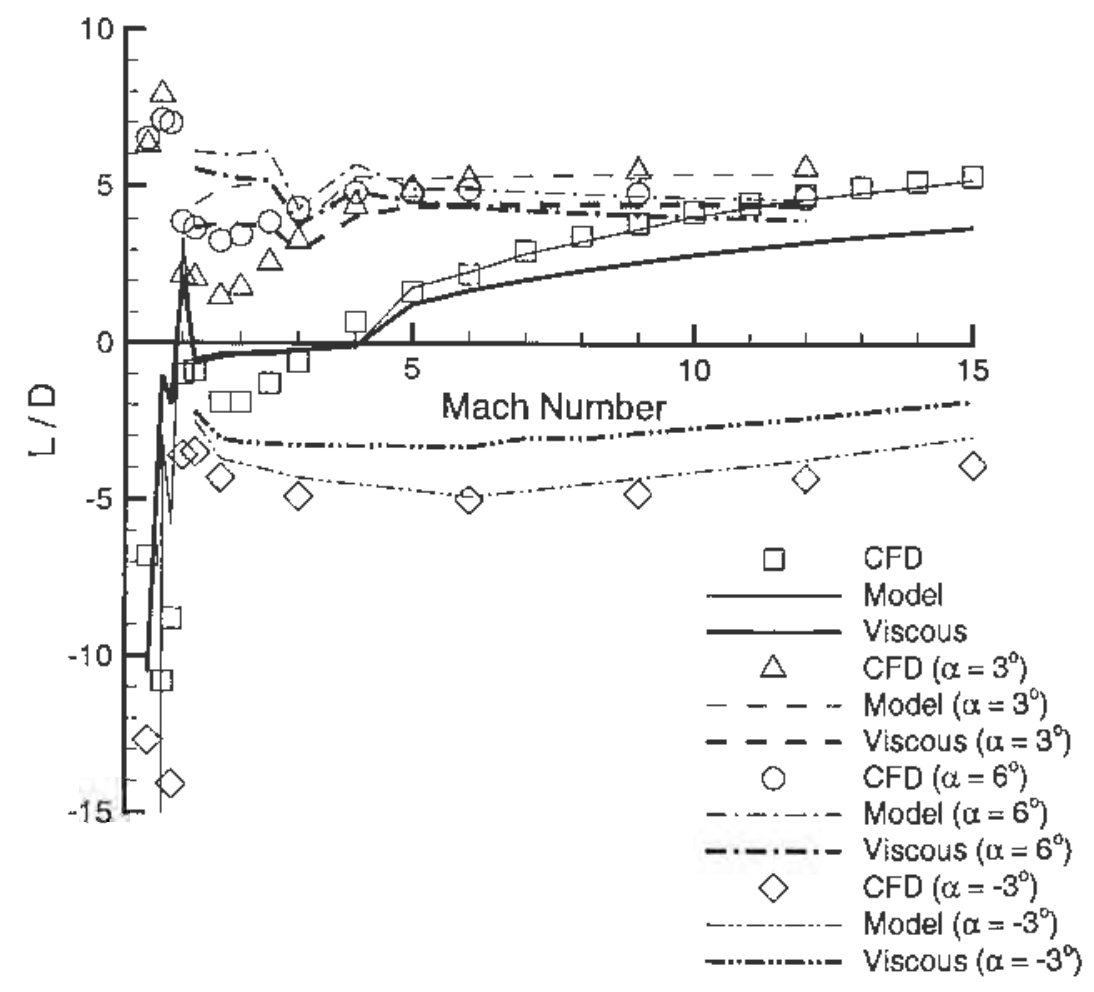

Figure 7.31: Inviscid and viscous airframe $L / D$ as a function of Mach number.

lincar function of the Mach number ranging from $300 \mathrm{~K}$ at Mach 1 to $1200 \mathrm{~K}$ at Mach 12 , staying constant at $1200 \mathrm{~K}$ for Mach numbers above 12 . With the increasc in drag because of viscosity, the magnitude of $L / D$ is seen to decrease for all Mach numbers and angles of attack. Viscosity is also seen to have the largest impact on airframe $L / D$ for zero and negative angles of attack. At the (lesign Mach number, the magnitude of $L / D$ was calculated to decrease by $36 \%$ for $-3^{\circ}$ angle of attack, $29 \%$ for zero angle of attack, $17 \%$ for $3^{\circ}$ angle of attack, and $13 \%$ for $6^{\circ}$ angle of attack. For positive angles of attack, the expansion on the upper surface tends to substantially lower viscous drag. For negative angles of attack, How compression on the upper surface drives up pressure and tcrmperature, thus increasing viscous losses. At the same time, compression still occurs on the lower surface so that viscous drag is not as affected. Hence, it is 
concluded from Fig. 7.31 that an accurate approximation of viscous losses in the high-speed regime is crucial in predicting vehicle performance.

The inviscid and viscous airframe lift-to-drag ratio is plotted as function of angle of attack in Fig. 7.32 for the methods of Table 7.2. Several trends are observed in the data plotted in Fig. 7.32. The maximum $L / D$ for all Mach numbers plotted occured for positive angle of attack. The maximum $L / D$ tends to shift toward higher angle of attack as Mach number decreases. As Mach number decreases, the $L / D$ tends to remain relatively constant near the maximum $L / D$ for increasing angle of attack. For a range of Mach numbers between 6 and 15, the $L / D$ remains very constant for an angle of attack in the $3^{\circ}$ to $3.5^{\circ}$ range. As Mach number decreases, the zero lift angle of attack shifts toward positive angle of attack. For the viscous solutions, the value of maximum $L / D$ remains roughly the same for Mach numbers between 6 and 15 . The slope in $L / D$ is much steeper for negative angles of attack than positive (as observed in Fig. 5.3 in Section 5.1).

\subsection{Engine-Airframe Integrated Performance}

\subsubsection{Scramjet Integration}

The vehicle models are integrated with the scramjet engine model developed in Chapter 3. A new Mach 12 vehicle design is selected (shown in Fig. 7.33) to demonstrate the capabilities of the model to predict powered performance. This geometry was used since it could produce positive thrust at both Mach 8 and Mach 12, but does not imply an optimum solution. The example also does not include moment trim, thus the effects of control surfaces on the performance of the vehicle are not accounted. The geometric properties for the example vehicle are shown in Table 7.3. All performance numbers assume a constant value for 


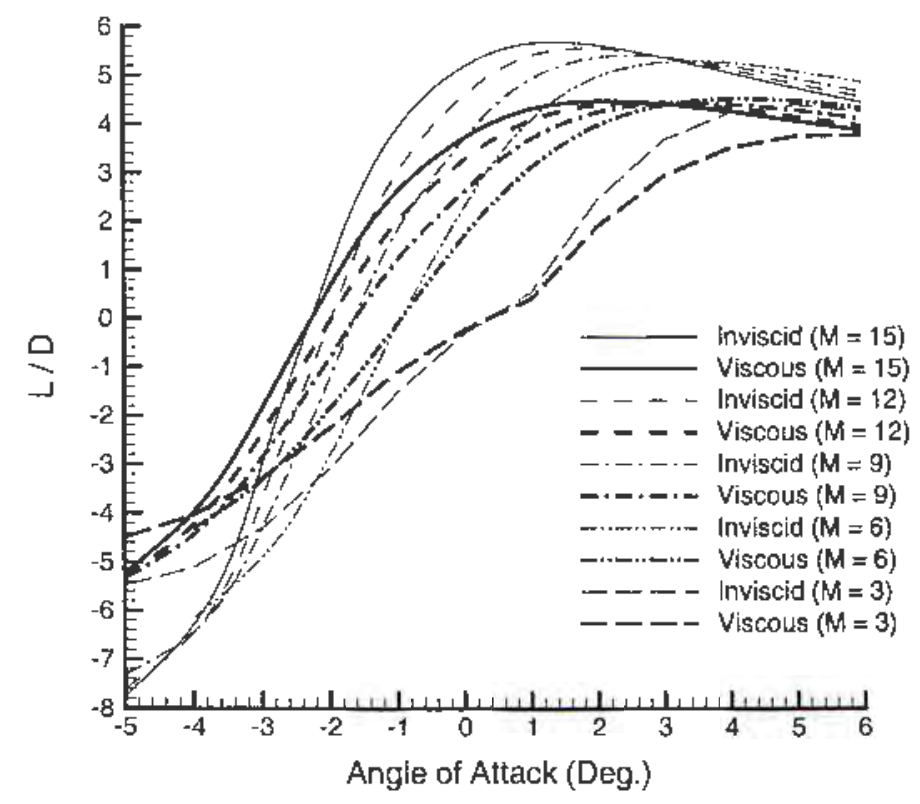

Figure 7.32: Inviscid and viscous airframe $L / D$ as a function of angle of attack.
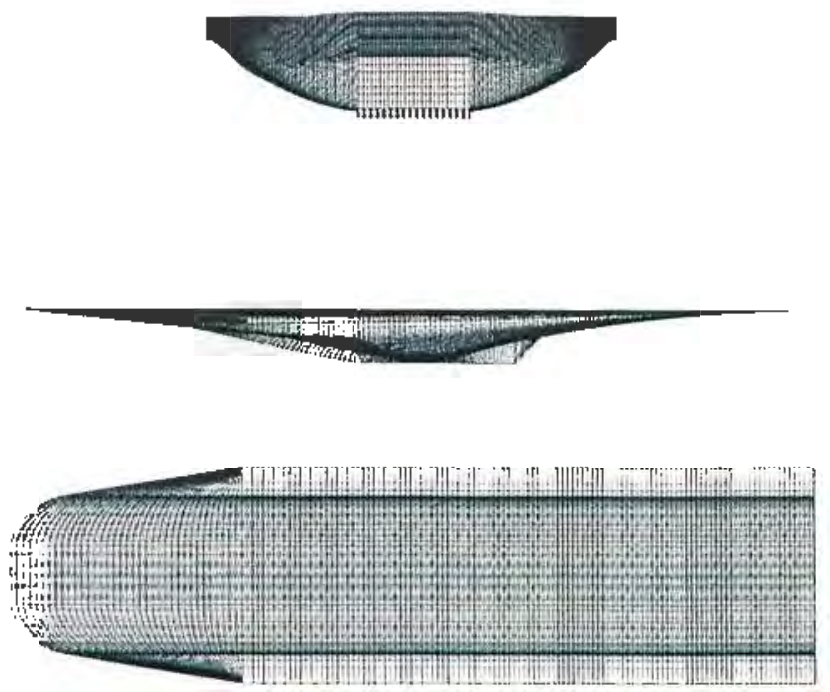

Figure 7.33: Three-view of Mach 12 scramjet-powered example vehicle. 


\begin{tabular}{cc}
\hline \hline Length (m) & 76.8 \\
Width (m) & 20.5 \\
Heigltt (m) & 5.2 \\
Volume $\left(\mathrm{m}^{3}\right)$ & 2392 \\
Planform Area $\left(\mathrm{m}^{2}\right)$ & 429.2 \\
Max Cross-Sectional Area $\left(\mathrm{m}^{2}\right)$ & 71.6 \\
Inlet Ramp Angles & $1.07^{\circ}$ \\
Shock Angle & $9.26^{\circ}$ \\
Equivalence Ratio & 1 \\
\hline \hline
\end{tabular}

Table 7.3: Mach 12 example vehicle properties.

the equivalence ratio.

The viscous, powered L/D is plotted as a function of angle of attack in Fig. 7.34. The Mach 8 solution has a lower angle of attack limit to insure compression on the lower surface of the forebody. The Mach 10 and 12 solutions have lower angle of attack limits to insure that no shocks are swallowed into the engine. One noticable trait of these velicles is the difference in the derivative of lift to drag ratio with respect to angle of attack. The vehicle has a much steeper slope in lift to drag ratio at negative angles of attack than for positive angles of attack. This trend is due to shock compression on the upper surface for negative angles of attack, thus lowering the overall lift of the vehicle and $\mathrm{L} / \mathrm{D}$. For positive angles of attack, pressure drag on the lower surface is the prime contributor to lowering the L/D. This effect was seen in Fig. 7.32 as well as Fig. 5.3.

The vistous powered L/D in Fig. 7.34 is compared with the viscous, unpowered L/D plotted for a different Mach 12 vehicle in Fig. 7.32. The unpowered vehicle at Mach 1.2 had a maximum viscous $L / D$ of approximately 4 at an angle 


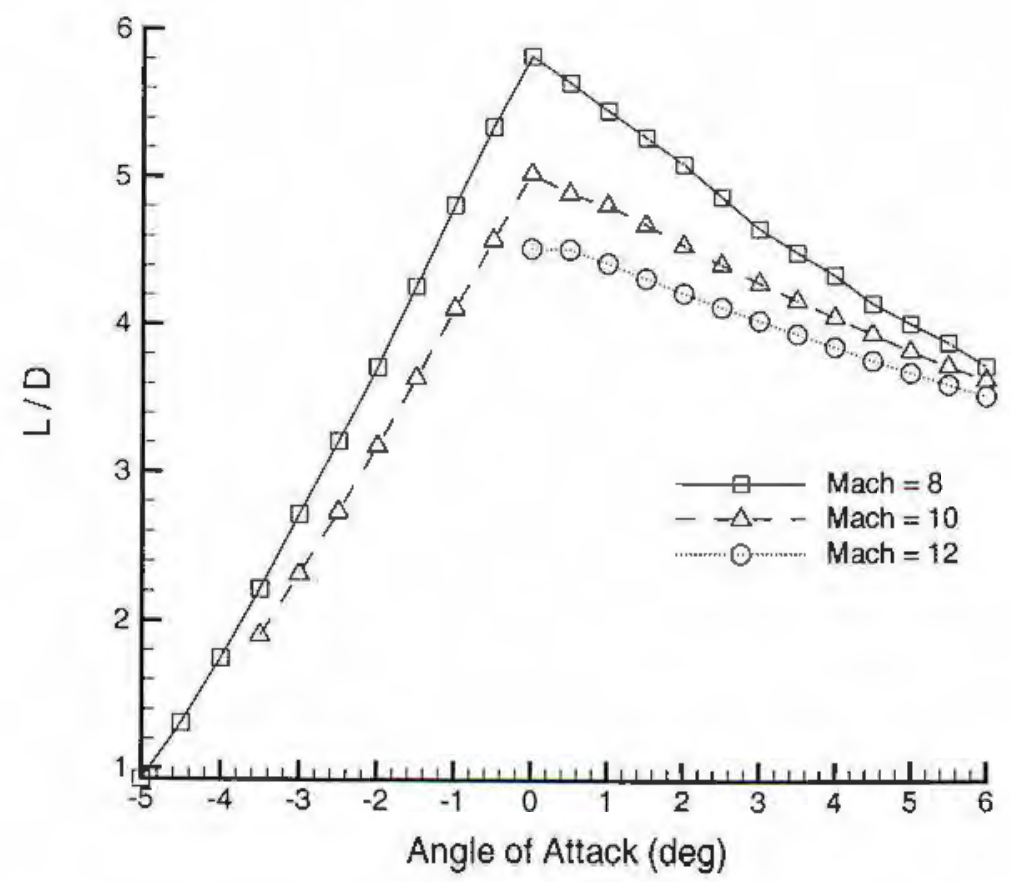

Figure 7.34: L/D vs. angle of attack for example Mach 12 vehicle $(\phi=1)$.

of attack of approximately $2.5^{\circ}$ and a maximum inviscid L/D of approximately 5.5 at an angle of attack of approximately $1.5^{\circ}$. The powered vehicle had a maximum viscous $\mathrm{L} / \mathrm{D}$ of approximately 6 at zero angle of attack. Considering that the vehicle geometries being compared are not identical, the effect of viscosity on the unpowered vehicle reduces the peak L/D by about $25 \%$. By adding the scramjet to the vehicle, the $\mathrm{L} / \mathrm{D}$ was improved to roughly the same performance as the inviscid geometry. Hence, the addition of the engine to the vehicle geometry has the overall effect of recovering the aerodynamic performance of the fuselage lost due to viscosity. This implies that reducing the viscous losses on the vehicle fuselage could have an enormous pay-off in vehicle performance when engine-airframe integration is included.

Another conclusion that may be made from observing Figs. 7.34 and 7.32 is 
the effect of engine-airframe integration on the off-design angle of attack performance of the vehicle. The unpowered geometry had zero lift at an angle of attack of approximately $-2^{\circ}$. The powered geometry had positive lift down to its angle of attack limit of $-5^{\circ}$. These results clearly demonstrate the amount of lift the vehicle obtains from its engine. The high pressure engine exhaust is expanded over the rear portion of the vehicle. Enough lift is generated from the engine exhaust to lower the zero-lift angle of attack by at least $3^{\circ}$. By extending the zero-lift angle of attack, the vehicle essentially has more maneuverability along its trajectory, allowing for larger flight path angles and greater mission flexibility.

Angle of attack on the powered geometry (for all Mach numbers solved) also had the effect of lowering L/D. This is a distinct difference from the unpowered geometry, which typically had maximum L/D for some positive angle of attack. At the on-design condition, the maximum amount of compression occurs on the forebody. With off-design angle of attack, a portion of the flow compressed by the engine inlet will be spilled to avoid shock swallowing. Thus, the engine is most efficient at zero degrees angle of attack (minimum spillage). By placing the vehicle at an angle of attack, the L/D of the fuselage increases (see Fig. 7.32), but the effects from the engine will lower the L/D of the engine-airframe integrated vehicle. This is another example of the effect of the engine on the vehicle performance.

The effective specific impulse of the vehicle is plotted as a function of angle of attack in Fig. 7.35. The vehicle has positive net thrust (i.e., positive $I_{\mathrm{sp}_{\mathrm{eff}}}$ ) for a range of Mach numbers between 8 and 12 and a limited range of angles of attack. High $I_{\mathrm{sp}_{\mathrm{eT}}}$ at negative angle of attack is primarily due to the lift vector being shifted in the direction of thrust. This effect has its limits since the increased pressure on the upper surface due to shock compression increases the overall drag of the vehicle. Also, as angle of attack gets more negative, less compression 


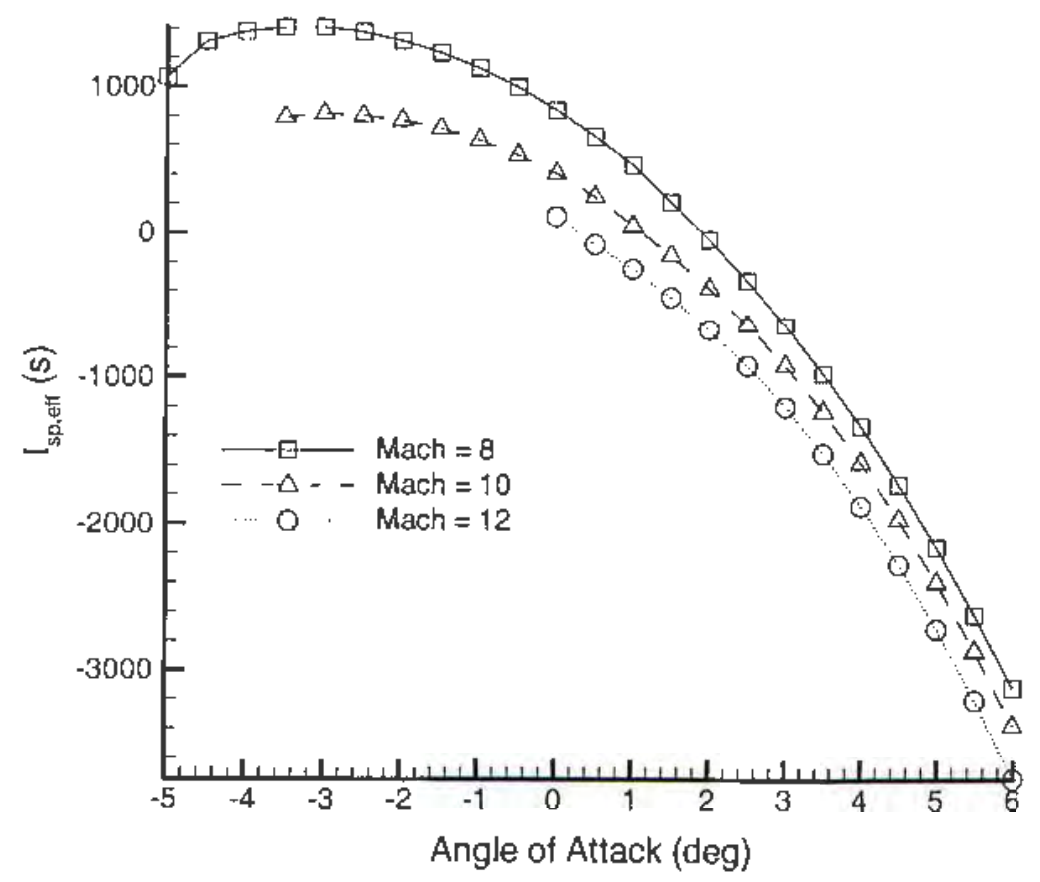

Figure 7.35: $I_{\mathrm{sp}_{\mathrm{uff}}}$ vs. angle of attack for example Mach 12 vehicle $(\phi=1)$.

occurs prior to entering the engine, resulting in a lower thrust from the engine.

An interesting result comparing Figs. 7.34 and 7.35 is that for nogative angles of attack, specific impulse is greater, but the L/D has a steep negative slope. Thus, a trade-off exists between high thrust production and high lift production. For positive angles of attack (where the vehicle would want to fly to minimize losses in L/D), the vehicle has poor thrust performance. These results may be geometry dependent (since the vchicle was designed solely for positive thrust between Mach 8 and Mach 12), and could be rather different if a different vehicle design is used. 

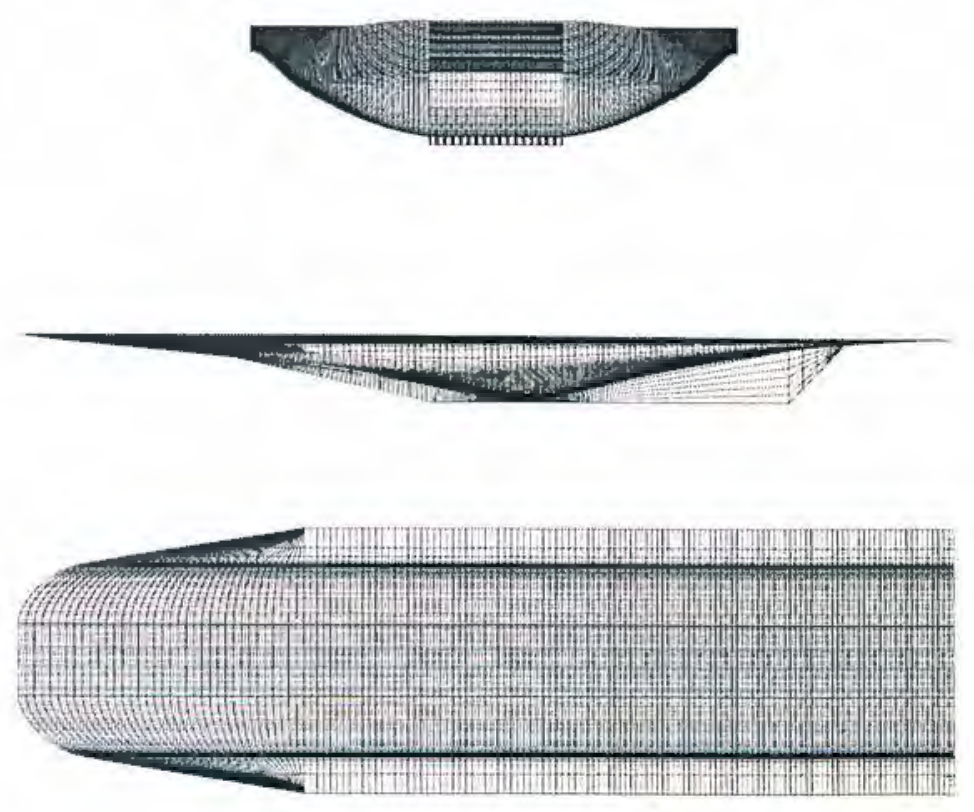

Figure 7.36: Three-view of modified Mach 12 ramjet-powered vehicle.

\subsubsection{Ramjet Integration}

The ramjet example provided in Chapter 3 is now integrated with an airframe geometry. The airframe selected for this analysis was the same airframe geometry shown in Fig. 4.27 with two modifications and is shown in Fig. 7.36. The modifications made to the vehicle are a reduction in vehicle length of approximately $4 \mathrm{~m}$ and an increase in engine cowl length of approximately $11.5 \mathrm{~m}$. These modifications were necessary to keep the last characteristic of the engine plume from interacting with the vehicle nozzle. Observing the vehicle geometry in Fig. 7.36, it is apparent that the engine cowl is too long because of viscous losses and weight considerations. The example vehicle in Fig. 7.36 implies no optimality, but is provided as a demonstration of the application of the ramjet engine model integrated into the vehicle model.

The on-design $L / D$ of the original geometry was 4.33 , compared to the on- 


\begin{tabular}{cc}
\hline \hline On-design $L / D$ & 3.48 \\
Off-design $L / D$ at Mach 5 & 5.39 \\
Volicle specific impulse & $5384 \mathrm{~s}$ \\
Engine specific impulse & $4948 \mathrm{~s}$ \\
Effective, installed, engine specific impulse & $2584 \mathrm{~s}$ \\
Ideal engine specific impulse & $4942 \mathrm{~s}$ \\
\hline \hline
\end{tabular}

Table 7.4: Performance of modified Mach 12 ramjet-powered vehicle.

clesign $L / D$ of the modified geonetry of 3.48 (a reduction of approximately $20 \%$ ). For the off-design conditions where a ramjet would be necessary, the vehicle in Fig. 7.36 is flown at the same conditions as the ramjet example in Section 3.3: frestream Mach number of 5 and an altitude of $24.7 \mathrm{~km}$. The resulting offdesign performance of the velicie is listed in Table 7.4. The $L / D$ of the ramjet powered vehicle is 5.39 (using the lyppersonic modeling for the aerodynamics on the fuselage developed in Chapter 5). This result is lower than the scranjetpowered vehicle of Fig. 7.33 flying at an off-design velocity of Mach 8. Thus, the vohiclo gcometry las roon for improvement.

The: vehicle specific impulse is $5384 \mathrm{~s}$, the engine specific impulse is 4948 $s$, and the effoctive, installed engine specific inpulse was $2584 \mathrm{~s}$. The vehicle specific impulse is a micasure of the total vehicle thrust, the engine specific impulse is a measure of the total engine thrust, and tine effective, installed engine specific impulse is a measure of the net thrust of the engine. For all specific impulses listed, tho engine is considered to consist of the forebody and intet ramp compressions, engine sidewalls, engine struts, and the entire engine and vehiche nozzle. 
The ideal specific inpulse of a ranjet may be calculated by

$$
I_{\mathrm{sp}}=\frac{T h}{\dot{m}_{j} g}=\frac{M_{\infty} \sqrt{\gamma R T_{\infty}}}{\int g}\left[(1+f) \sqrt{\frac{T_{0, e}}{T_{\infty}}}\left(1+\frac{\gamma-1}{2} M_{\infty}^{2}\right)^{-\frac{1}{2}}-1\right]
$$

where $T_{g, c}$ is the total temperature at the exit of the combustor calculated by

$$
T_{o, e}=\frac{T_{\infty}\left(1+\frac{r-1}{2} M_{\infty}^{2}\right)+\frac{\int Q_{r}}{c_{p}}}{1+f}
$$

and $Q_{r}$ is the lower heating valuo of the fuel $\left(119,954 \mathrm{~kJ} / \mathrm{kg}\right.$ for hydrogen $\left.{ }^{38}\right)$. The assumptions made in the derivation of Eq. 7.3 include: constant $\gamma$, constant $c_{p}$ isentropic compression and expansion, constant pressure combustion, no Thayleigh losses, exit pressure is equivalent to frcestream pressure (idcal expansion), neglect fuel entlalpy, and a combustion efficiency of $100 \%$. With this analysis, the ideal ramjet specific impuise for the abowe example is calculated to be $4942 \mathrm{~s}$ (assuming $\gamma=1.4$ and $c_{p}=1.0045 \mathrm{~kJ} / \mathrm{kg}$ ). The ideal case was found to overpredict the effective, installed, engine specific impulse by nearly a factor of 2. However, this crror is expected considering the assumptions using the ideal specific impulse and the inclusion of the forebody, inlet ramps and nozzle in the analysis of the effective, installed engine specific impulse.

\subsection{Scramjet-Powered Trajectory Performance}

The scramjet-powered example vehicle in Fig. 7.33 was flown through a non-optimal, quadratic trajectory as shown in Fig. 7.37. Starting conditions for the vehicle were an altitude of $30.7 \mathrm{~km}$., freestream Mach number of 8 , and a constant equivalence ratio of 1 . Two cases werc run for the given trajectory, corresponding to a vehicle with $40 \%$ of its fucl remaining at the beginning of the trajectory and one with $50 \%$ of its fuel remaining. These cases were selected to bracket the amount of fuel required to reach the altitude and Mach number solucted. 


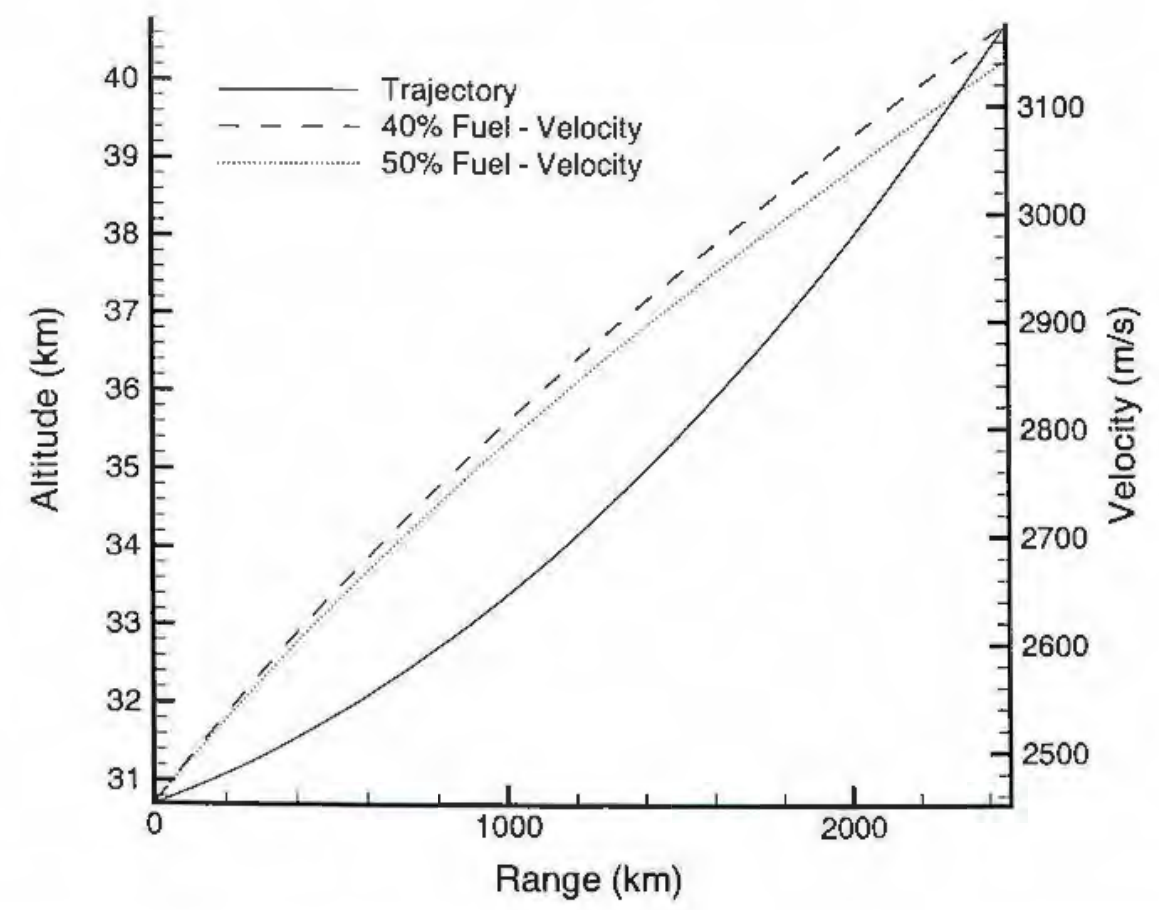

Figure 7.37: Trajectory and velocity profile as a function of range and percent fuel remaining.

The resulting velocity profile for each case is also plotted in Fig. 7.37. The difference in starting mass resulted in only a $1 \%$ increase in velocity over the given trajectory. For both cases shown, the vehicle continued to accelerate through the entire given trajectory. Thus, the models developed herein are capable of taking a scramjet powered vehicle through a flight trajectory to obtain vehicle performance. However, the trajectory plotted in Fig. 7.37 is not an optimal trajectory, so future study into the trajectory performance of these types of vehicles for realistic flight trajectories should be investigated.

The Mach number profile for the example vehicle flown through the trajectory plotted in Fig. 7.37 is shown in Fig. 7.38. The $40 \%$ fuel remaining case resulted in a final Mach number of 9.75 while the $50 \%$ case resulted in a hnal Mach 


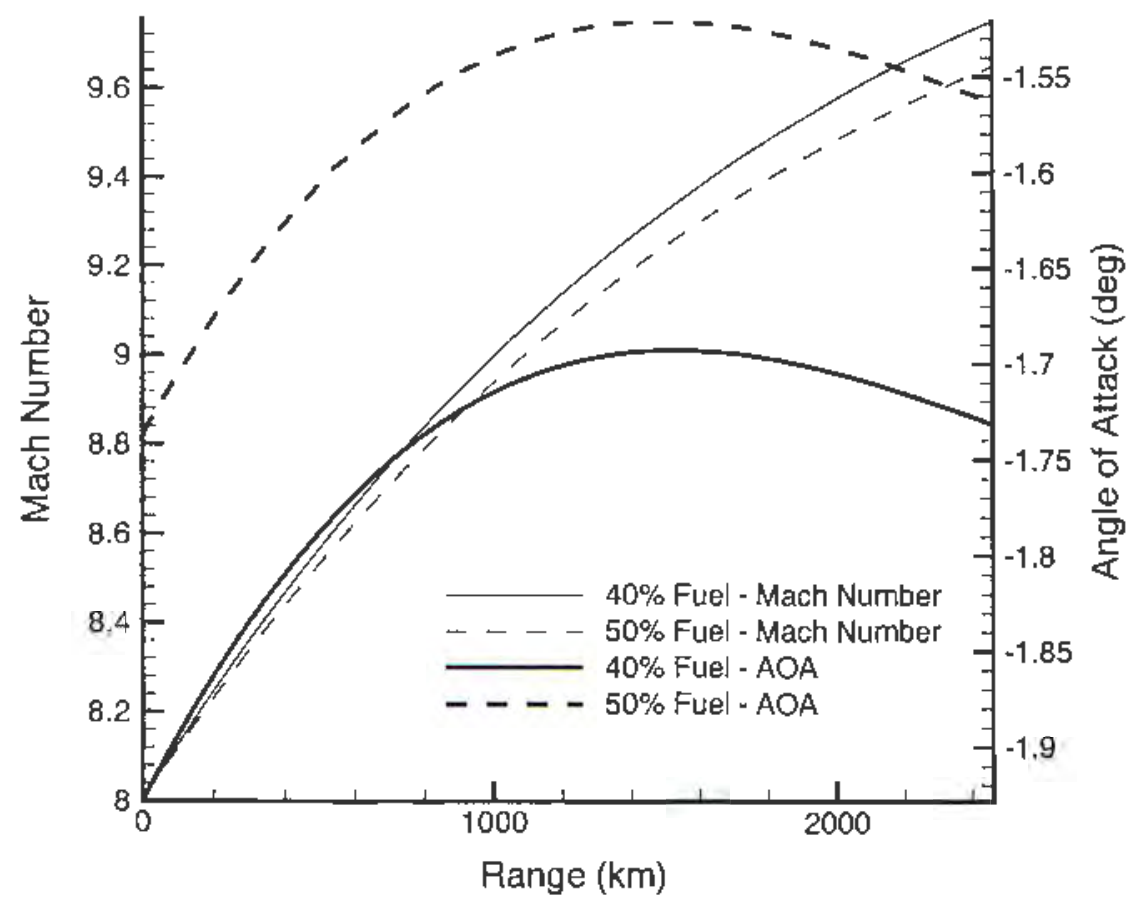

Figure 7.38: Mach number and angle of attack profile as a function of range and jercent fuel remaining.

number of 9.65 . Thus, the effect of $10 \%$ more fuel mass resulted in a reduction of final Mach number by only $1 \%$, showing that the acceleration of the vehicle was not strongly dependent on the vehicle mass. However, this again may be a function of the trajectory selected and may be resolved by a more detailed trajectory analysis.

The angle of attack profile through the flight trajectory is also presented in Fig. 7.38. For botil cases, a maximum in angle of attack was found at a range location near $1550 \mathrm{~km}$. The deviation in angle of attack across the trajectory was seen to be cuite small $\left(\sim 0.3^{\circ}\right)$. This region of angle of attack is seen to be close to the maximum $I_{s p_{\varepsilon f f}}$ for Mach numbers between 8 and 10 (see Fig. 7.35), and to have a relatively constant value of $L / D$ between 3.5 and 4 (see Fig. 7.34). 
The fuel mass consumed for the $40 \%$ fucl remaining case was $14273 \mathrm{~kg}$. of $\mathrm{H}_{2}$ compared with $14552 \mathrm{~kg}$ of $\mathrm{H}_{2}$ for the $50 \%$ fuel remaining case, for the same trajectory. Since the $40 \%$ case had less fuel, it had to do less work to get to the same altitude as the $50 \%$ chse, hence having lower fuel consumption. 


\section{Chapter 8}

\section{Summary and Conclusions}

The preceding chapters developed a methodology for predicting the on- and off-design aerodynamic behavior of hypersonic, waverider-based, RBCC engineairframe integrated vehicles. The on-design fuselage methodology uses the osculating cones waverider generation technique to develop the vehicle forebody, followed by a cubic-spline with continuous slope and trailing edge closure. The on-design RBCC engine methodology includes prediction of compression conditions between injector struts as well as a quasi-one-dimensional finite-rate chemistry combustor model.

Three aerodynamic models were developed for the off-design aerodynamic performance of the vehicle fuselage: a hypersonic model, a supersonic model, and a transonic model. Each model is validated using three-dimensional, inviscid CFD computations over the vehicle fuselage. The hypersonic model tended to predict the flowfields the best, having Mach number limits in the 4 to 5 range for the Mach 12 vehicle solved. The supersonic model tended to underpredict fuselage compression, but had applicability in the Mach 3 to 6 range for the Mach 12 vehicle solved. The transonic model tended to predict zero angle of attack solutions best, with higher errors for supersonic solutions. The off-design models were improved by blending them together in such a way that a relatively 
smooth transition occurs through all Mach numbers solved. The results gave good predictions of trends from Mach 0.4 up to 15 and for angles of attack between $-3^{\circ}$ and $6^{\circ}$, with a tendency for increased error with decreasing Mach number.

The engine model consisted of two portions: a strut/strut compression model and a combustor flowfield model. The strut/strut compression model was validated using two-dimensional, inviscid CFD solutions and was found to both qualitatively and quantitatively predict the compression flowfield better than assuming quasi-1D flow. The area-averaged properties exiting the strut/strut compression system are then applied as the incoming properties to the combustor model. The combustor flow-field model used quasi-1D methodology and included finite-rate chemistry. The combustor model in scramjet mode was validated by comparison to two experimental analyses of hydrogen-fueled scramjet designs. The model predicted fuel ignition as well as the pressure along the thrust surface of the engine. The combustor model was also demonstrated to operate in ramjet mode, where a thermal throat was established to transition the flow from subsonic to supersonic.

An analysis of the aerodynamic off-design models compared to the computational results brought up several interesting findings with regards to the lift of the vehicle. The lift coefficient of the vehicle tended to have a linear profile with respect to angle of attack for all Mach numbers solved (from 0.4 to 15). The lift coefficient was also observed to be rather insensitive to freestream Mach number. For the example Mach 12 vehicle solved, the zero-lift Mach number at zero angle of attack was 3.5. Further investigation showed that these class of geometries have poor low-speed lift performance. These results imply that some additional means of producing high lift at low-speeds (such as wings, canards, engine thrust, reshaping vehicle planform area, etc.) will be necessary. Low-speed performance of the vehicle may be improved by incorporating low-speed effects into the initial 
design and subscouent optimization of the vehicle geometry.

The analysis of the drag performance of the vehicle also resulted in several interesting conchusions. A curvefit of the hypersonic, inviscid drag polar of the geometry showed the value of the exponent of lift cocficient to be approximately 1.75 for Mach numhers ranging from 6 to 15. This value of the exponent happens t.o be the average value of the exponent for Newtonian flow (1.5) and tangentwedge flow (2). The minimum drag coefficient had a Mach number dependency shifting from negative angles of attack in the hypersonic regime to essontially zere angle of attack for low supersonic Mach numbers. The maximum inviscid cl rag coefficient was observed to oceur for a Mach number of 1.2 for all angles of attack solved. Finally, the viscous transonic drag rise was observed with the mortels developed and was found to have a maximum at Mach 1.2 for the blended aerodynamir: model.

The scramjet engine model was incorporated into the hypersonic vehicle model to investigate the effects of engine-airframe integration on the aerodynamic performance of the vchicle. A cornparison was made between the clcan, unpowered vehicle fusclago and a separate, but similar, vehicle geometry that had the RBCC engine integrated into the airframe. Powered engine integration had the effect of balancing ont the viscous losses on the unpowered vehicle fuselage. The engine also had a beneficial effect on zero-lift angle of attack, lowering it substantially due to the additional lift devoloped by the engine exhaust expanding over the rear of the vehicle. A trajectory analysis was also performed to demonstrate the ability to apply the vehicle and engine aerodynamic models to an application that depends on aerodynamic data to predict perfornance. The results showed that the models developed smooth sulutions for the trajectory selected.

The rantijet engine model was incorporated into a modified Mach 12 vehicle model, and demonstrated the ability to predict performance of the engine- 
airframe integrated vehicle operating in ramjet mode. The vehicle was modified by shortening its total length and increasing its engine cowl length to prevent the last characteristic of the engine plume from interacting with the nozzle surface. The rosults showed that the specific impulse of the engine-airframe integrated ramjet operated at aloont $50 \%$ of the specific inpulse of an ideal ramjet. The analysis showod that future velicle desigis must also include ramjet considerations in the design of the engine and airframe geometries. 


\section{Chapter 9}

\section{Future Work}

Tho anrodynamic methods presented in this dissertation are believed to be the first steps toward developing off-design prediction techniques for waveriderbasod fuselages, rocket-based combined cycle engines, and their integration. Much insight has been gained on many aspects of the methodology that could use innprovement or further investigation. Recommendations for future work based on the results of this dissertation will be presented in the following sections.

\subsection{Vehicle Definition}

- The goometry selected for this dissertation had continuous slope, but did not have continuity in the second and third derivatives. The transonic methods presented in Chapter 4 required smooth second and third derivatives of the geonetry, hence a spline was performod on the geometry to meet these restrictions. Future vehicle designs would benefit from defining a fusclage shaje with continuous first, second, and third derivatives. This can be accomplished by using a single seventh-order polynomial that would act as onc curve for the entire fusclage (as opposed to the two cubics used in this dissertation). The polynomial coefficients may then be defined from 
the location, slone, second, and third derivatives at the waverider/fuselage intersection location, the location and slope at the outlet of the engine, and the location and slope at the end of the vehicle.

- The top surface of the waverider geometry was assumed to be parallel to freestream. Future vehicle geometries may benefit from adding a compression angle to the upper surface to increase volume.

- A design Mach number of 12 was assumed for the vehicle design used to validate the aerodynamic models. The effects of raising and lowering the vehicle design Mach number on the accuracy of the off-design models should be performed.

- The vehicle definition would greatly benefit from inclusion of low-speed considerations into the initial design and optimization of the geometry. The solution to the low-speed problems of the geometry may be as simple as developing a better planform geometry. However, it is believed that the shaping of the rear of the vehicle may also benefit the low speed performance. The lift of the vehicle may be increased dramatically if higher pressures can be generated on the rear portion of the vehicle. This could be easily obtained by reducing the amount of expansion on the rear of the vehicle. Further study is needed to verify both of these claims.

\subsection{Off-Design Aerodynamic Models}

- The results of Chapter 6 showed that the model in most need of improvement is the supersonic off-design method. A blending of the oblique shock relations and the axisymmetric solutions may solve the underprediction of pressure in the compression region. 
- Angle of attack solutions must also be developed for the subsonic and near sonic solutions. Improvements are also necessary for the low supersonic ranger.

- The effects of yow at all Mach numbers has not been investigated in this dissertation. The aerodynamic performance of these vehicles for small angles of yaw are necessary for prediction of stability and control. An analytical means of predicting the aerodynamic performance at yaw would be a very valuable contribution to the field.

- The offects of viscosity on the aerodynanic distribution at transonic speeds was ignored for this dissertation. An accurate prediction of the transonic cing rise of the geometry will depend on modeling the interaction between the transonic shock on the body and the boundary layer.

- A major source of crror for the off-design aerodynamic models is the assumption of no cross-flow on the geonetry. A means of predicting the cross-flow on the geometry should be developed to reduce the error in the current methodology.

- For this disscrtation, it was assumed that the engine would not affect the fusclage flow field, and was removed for analysis. In reality, the interference effects from the engine will liave a large effect on the aerodynamics of the fuselage. These effects will need to be incorporated into the off-design modeling to have an accurate prediction of the off-design beliavior of these vehicles.

- The trajectory performance investigated in this dissertation was limited to a quadratic profile. An optimized trajectory (or a coupling of the vehicle and the trajectory in an optimizer) should be investigated to obtain a better performance cstimation along a realistic flight profile. 


\subsection{RBCC Engine Model}

- A major source of error in the engine modeling is the assumption of no viscosity in the compression system prior to the combustor. Threedimensional effects such as corner flow will inevitably alter the compression profile. Other effects such as shock wave/boundary layer interactions will substantially affect the compression between the struts.

- Although the models developed herein did not consider shocks swallowed by the inlet, it is inevitable that a real vehicle will have to deal with shock waves entering the engine. These effects should be incorporated into the engine model.

- The topic of engine starting and unstarting at high-speeds was not addressed. This is a crucial consideration for vehicles transitioning from a ramjet to a scramjet. Future work should consider such effects in the design of the engine compression system.

- The fuel mixing profile in this study was a curvefit based on experimental data. A better, more realistic mixing model (which includes dependency on flowfield properties) will be a valuable addition to the engine modeling.

- Fuels other than cryogenic hydrogen should be considered for both engine modeling and engine-airframe integration.

- The development of the airframe and engine models allowed for a straightforward analysis of ramjet-powered flight using the combustor code. The ability to model ramjet behavior was established, but two key components of a ramjet system were ignored for this study: a variable inlet geometry and a flameholder. The performance of the $\mathrm{RBCC}$ engine in ramjet mode 
discussed in this work may be improved upon by the addition of these technologies to the engine model. 


\section{Appendix A}

\section{Secant Method}

The secant method is designed to solve for the zero of a nonlinear, algebraic function $f$. Solution begins by selecting two values for the dependent variable $x$ and solving for the value of $f$ at each value. An crror value is then selected to determine the highost value of $f$ acceptable, which serves as the convergence criteria. From the values of $x_{1}, x_{2}, f_{1}$, and $f_{2}$, a new value for $x$ is found

$$
x_{3}=x_{2}-\frac{x_{2}-x_{1}}{1-\frac{f_{1}}{f_{2}}}
$$

I'he value of $a_{3}$ is then used to solve for an updated value of $f$. If the absolute value of the updated value of $f$ is still higher than the error value selected, then the variables are updated as follows

$$
\begin{aligned}
& x_{1}=x_{2} \\
& f_{1}=f_{2} \\
& x_{2}=x_{3} \\
& f_{2}=f_{3}
\end{aligned}
$$

From the updated value of the variables, a new value of $x_{3}$ is solved using Eq. A.1. This process is iterated until the desired error has been reached. 


\section{Appendix B}

\section{Hydrogen/Air Reaction Mechanism}

The hydrogen/air reaction mechanism used in this study was proposed by Jachimowski "5. Jachimowski's mechanism (shown in Table B.1) contains 14 spocies and 33 reactions, includes nitrogen chemistry, and was specifically developed for application to high-speed combustion modeling. Table B.1 contains the information to write the rate coefficionts in Arrhenius form:

$$
k=A T^{n} \exp \left(\frac{-E_{a}}{R_{u} T}\right)
$$

where $A$ is the frequency or pre-exponential factor (units of mole-cm-s-K), $n$ is an exponent, and $E_{a}$ is the activation energy (units of cal/mole). For three body reactions, the reaction rate coefficients nay be written as:

$$
k=A T^{n} \exp \left(\frac{-E_{a}}{R_{i u} T}\right)[X]^{a}[Y]^{b}[Z]^{e}
$$

where $[X],\left[Y^{*}\right]$, and $[Z]$ are molar concentrations of species $X, Y$, and $Z$, respectively. Third-body efficiencies are (referring to Table B.1): reaction 6) $\mathrm{H}_{2} \mathrm{O}=$ 6.0, reaction 7) $\mathrm{H}_{2}=2.0$ and $\mathrm{H}_{2} \mathrm{O}=6.0$, reaction 8) $\mathrm{H}_{2} \mathrm{O}=5.0$, reaction 9) $\mathrm{H}_{2}$ $=2.0$ and $\mathrm{H}_{2} \mathrm{O}=16.0$, and reaction 19$) \mathrm{H}_{2} \mathrm{O}=15.0$. 
Table B.1: Hydrogen reaction mechanism by Jachimowski [Ref. 61].

\begin{tabular}{|c|c|c|c|c|}
\hline & Reaction & $\bar{A}$ & $n$ & $E_{a}$ \\
\hline 1 & $\mathrm{H}_{2}+\mathrm{O}_{2} \rightleftharpoons 2 \mathrm{OH}$ & $1.7 \mathrm{E} 13$ & 0 & 48,000 \\
\hline 2 & $\mathrm{H}+\mathrm{O}_{2} \rightleftharpoons \mathrm{OH}+\mathrm{O}$ & 2.6E14 & 0 & 16,800 \\
\hline 3 & $\mathrm{O}+\mathrm{H}_{2} \rightleftharpoons \mathrm{OH}+\mathrm{H}$ & $1.8 \mathrm{E} 10$ & 1 & 8,900 \\
\hline 4 & $\mathrm{OH}+\mathrm{H}_{2} \rightleftharpoons \mathrm{H}_{2} \mathrm{O}+\mathrm{H}$ & $2.2 \mathrm{E} 13$ & 0 & 5,150 \\
\hline 5 & $2 \mathrm{OH} \rightleftharpoons \mathrm{H}_{2} \mathrm{O}+\mathrm{O}$ & $6.3 \mathrm{E} 12$ & 0 & 1,090 \\
\hline 6 & $\mathrm{H}+\mathrm{OH}+\mathrm{M} \rightleftharpoons \mathrm{H}_{2} \mathrm{O}+\mathrm{M}$ & $2.2 \mathrm{E} 22$ & -2 & 0 \\
\hline 7 & $2 \mathrm{H}+\mathrm{M} \rightleftharpoons \mathrm{H}_{2}+\mathrm{M}$ & 6.4E17 & -1 & 0 \\
\hline 8 & $\mathrm{H}+\mathrm{O}+\mathrm{M} \rightleftharpoons \mathrm{OH}+\mathrm{M}$ & $6 \mathrm{E} 16$ & -0.6 & 0 \\
\hline 9 & $\mathrm{H}+\mathrm{O}_{2}+\mathrm{M} \rightleftharpoons \mathrm{HO}_{2}+\mathrm{M}$ & $2.1 \mathrm{E} 15$ & 0 & $-1,000$ \\
\hline 10 & $\mathrm{HO}_{2}+\mathrm{H} \rightleftharpoons \mathrm{H}_{2}+\mathrm{O}_{2}$ & $1.3 \mathrm{E} 13$ & 0 & 0 \\
\hline 11 & $\mathrm{HO}_{2}+\mathrm{H} \rightleftharpoons 2 \mathrm{OH}$ & $1.4 \mathrm{E} 14$ & 0 & 1,080 \\
\hline 12 & $\mathrm{HO}_{2}+\mathrm{H} \rightleftharpoons \mathrm{H}_{2} \mathrm{O}+\mathrm{O}$ & $1 \mathrm{E} 13$ & 0 & 1,080 \\
\hline 13 & $\mathrm{HO}_{2}+\mathrm{O} \rightleftharpoons \mathrm{O}_{2}+\mathrm{OH}$ & $1.5 \mathrm{E} 13$ & 0 & 950 \\
\hline 14 & $\mathrm{HO}_{2}+\mathrm{OH} \rightleftharpoons \mathrm{H}_{2} \mathrm{O}+\mathrm{O}_{2}$ & $8 \mathrm{E} 12$ & 0 & 0 \\
\hline 15 & $2 \mathrm{HO}_{2} \rightleftharpoons \mathrm{H}_{2} \mathrm{O}_{2}+\mathrm{O}_{2}$ & $2 \mathrm{E} 12$ & 0 & 0 \\
\hline 16 & $\mathrm{H}+\mathrm{H}_{2} \mathrm{O}_{2} \rightleftharpoons \mathrm{H}_{2}+\mathrm{HO}_{2}$ & $1.4 \mathrm{E} 12$ & 0 & 3,600 \\
\hline 17 & $\mathrm{O}+\mathrm{H}_{2} \mathrm{O}_{2} \rightleftharpoons \mathrm{OH}+\mathrm{HO}_{2}$ & $1.4 \mathrm{E} 13$ & 0 & 6,400 \\
\hline 18 & $\mathrm{OH}+\mathrm{H}_{2} \mathrm{O}_{2} \rightleftharpoons \mathrm{H}_{2} \mathrm{O}+\mathrm{HO}_{2}$ & $6.1 \mathrm{E} 12$ & 0 & 1,430 \\
\hline 19 & $\mathrm{H}_{2} \mathrm{O}_{2}+\mathrm{M} \rightleftharpoons 2 \mathrm{OH}+\mathrm{M}$ & $1.2 \mathrm{E} 17$ & 0 & 45,500 \\
\hline 20 & $2 \mathrm{O}+\mathrm{M} \rightleftharpoons \mathrm{O}_{2}+\mathrm{M}$ & $6 \mathrm{E} 17$ & 0 & $-1,800$ \\
\hline 21 & $2 \mathrm{~N}+\mathrm{M} \rightleftharpoons \mathrm{N}_{2}+\mathrm{M}$ & $2.8 \mathrm{E} 17$ & -0.75 & 0 \\
\hline 22 & $\mathrm{~N}+\mathrm{O}_{2} \rightleftharpoons \mathrm{NO}+\mathrm{O}$ & $6.4 \mathrm{E} 9$ & 1 & 6,300 \\
\hline 23 & $\mathrm{~N}+\mathrm{NO} \rightleftharpoons \mathrm{N}_{2}+\mathrm{O}$ & $1.6 \mathrm{E} 13$ & 0 & 0 \\
\hline 24 & $\mathrm{~N}+\mathrm{OH} \rightleftharpoons \mathrm{NO}+\mathrm{H}$ & $6.3 \mathrm{E} 11$ & 0.5 & 0 \\
\hline 25 & $\mathrm{H}+\mathrm{NO}+\mathrm{M} \rightleftharpoons \mathrm{HNO}+\mathrm{M}$ & $5.4 \mathrm{E} 15$ & 0 & -600 \\
\hline 26 & $\mathrm{H}+\mathrm{HNO} \rightleftharpoons \mathrm{NO}+\mathrm{H}_{2}$ & $4.8 \mathrm{E} 12$ & 0 & 0 \\
\hline 27 & $\mathrm{O}+\mathrm{HNO} \rightleftharpoons \mathrm{NO}+\mathrm{OH}$ & $5 \mathrm{E} 11$ & 0.5 & 0 \\
\hline 28 & $\mathrm{OH}+\mathrm{HNO} \rightleftharpoons \mathrm{NO}+\mathrm{H}_{2} \mathrm{O}$ & $3.6 \mathrm{E} 13$ & 0 & 0 \\
\hline 29 & $\mathrm{HO}_{2}+\mathrm{HNO} \rightleftharpoons \mathrm{NO}+\mathrm{H}_{2} \mathrm{O}_{2}$ & $2 \mathrm{E} 12$ & 0 & 0 \\
\hline 30 & $\mathrm{HO}_{2}+\mathrm{NO} \rightleftharpoons \mathrm{NO}_{2}+\mathrm{OH}$ & $3.4 \mathrm{E} 12$ & 0 & -260 \\
\hline 31 & $\mathrm{H}+\mathrm{NO}_{2} \rightleftharpoons \mathrm{NO}+\mathrm{OH}$ & $3.5 \mathrm{E} 14$ & 0 & 1,500 \\
\hline 32 & $\mathrm{O}+\mathrm{NO}_{2} \rightleftharpoons \mathrm{NO}+\mathrm{O}_{2}$ & $1 \mathrm{E} 13$ & 0 & 600 \\
\hline 33 & $\mathrm{M}+\mathrm{NO}_{2} \rightleftharpoons \mathrm{NO}+\mathrm{O}+\mathrm{M}$ & $1.16 \mathrm{E} 16$ & 0 & 66,000 \\
\hline
\end{tabular}




\section{References}

[1] McClinton, C. R., Voland, R. T., Holland, S. D., Engelund, W. C., White, J. T., and Pahle, J. W., "Wind Tunnel Testing, Flight Scaling and Flight Validation with Hyper-X," AIAA Paper 98-2866, 1998.

[2] Roche, J. M., and Kosareo, D. N., "Structural Sizing of a 25 000-lb Payload, Air-Breathing Launch Vehicle for Single-Stage-to-Orbit," NASA TM-2001210667, Jan. 2001.

[3] Nonweiler, T., "Aerodynamic Problems of Manned Space Vehicles," Journal of the Royal Aeronautical Society, Vol. 63, 1959, pp. 521-528.

[4] Nonweiler, T., "Delta Wings of Shapes Amenable to Exact Shock-wave Theory," Journal of the Royal Aeronautical Society, Vol. 67, 1963, pp. 3940.

[5] Takashima, N., Optimization of Waverider - Based Hypersonic Vehicle Designs. PhD thesis, University of Maryland, College Park, 1997.

[6] Eggers, A. J., Ashley, H., Springer, G. S., Bowles, J. V., and Ardema, M. D., "Hypersonic Waverider Configurations from the 1950's to the 1990's," in Proceedings of the First International Hypersonic Waverider Symposium, University of Maryland, College Park, Oct. 17-19 1990. 
[7] Kuchemam, D., The Aerodynamic Design of Aircraft. Pergamon Press, Oxford, England, 1978.

[8] Rasmussen, MI., Iyprersonic Flow. John Wiley and Sons, Inc, New York, Now York, 1994.

[9] Roe, P. L., "Theory of Waveriders," in AGARD Lecture: Aerodynamic Problems of IIypersonice Vethicles, 1972.

[10] Starkey, 12. P., Investigation of Air-Breathing, Hypersonic Missile Configurations Within Fatcrnal Box Constraints. PhD thesis, University of Maryland, October 2000.

[11] O'Neill, M. K. L., Optwmized Scramjet Engine Integration on a Waverider Airframe. PhD thesis, University of Maryland, College Park, 1992.

[12] Long, L. N., "Off-Design Performance of Hypersonic Waveriders," Journal of Aircraft, Vol. 27, July 1990, pp. 639-646.

[13] Vanmol, D., Heat Transfer Characteristics of Hypersonic Waveriders with Emphasis on the Leading Edge Effects. Master's thesis, University of Maryland, 1991.

[14] Tarpley, C., The Optimization of Engine - Integrated Hypersonic Waveriders with Stcady State Flight and Static Margin Constraints. PhD thesis, University of Marylint, College Park, 1995.

[15] Rudd, L., Longitudinal Dynumic Stability and Control of Mission-Oriented Hypersonic Waverider Vehicles. PhD thesis, University of Maryland, 2001.

[16] Bowcut, K. G., Optimization of Hypersonic Waveriders Derived from Cone Flows, Including Viscous Effects. PhD thesis, University of Maryland, 1986. 
[17] Kothari, A. P., and Bowcutt, K. G., "Leading Edge Optimization for Hypersonic Vehicles," in Proceedings of the First International Hypersonic Waverider Symposium, University of Maryland, College Park, Oct. 17-19 1990.

[18] O'Brien, T. F., Analysis of Power Law Shaped Leading Edges for Wuveriders Designed with Shock Atiachment. Master's thesis, University of Maryliand, College Park, Aug. 1998.

[19] O'Brien, T. F., and Lewis, M. J., "Power Law Shapes for Leading-Edge Blunting with Minimal Shock Standoff," Joumal of Spacecraft and Rockets, Vol. 36, Sep. - Oct. 1999, pp. 653-658.

[20] Venn, J., and Flower, J. W., "Shock Patterns for Simple Caret Wings," The Aeronautical Journal of the Royal Aeronautical Society, Vol. 74, Apr. 1970, pp. 339348 .

[21] Squire, L. C., "A Comparison of the Lift of Flat Delta Wings and Waveriders at High Angles of Incidence and High Mach Number," Ingenieur-Archiv, Vol. 40 , No. 5, 1971, pp. 339-352.

[22] Townend, L. H., "Some Design Aspects of Space Shuttle Orbiters," in Progress in Aerospace Sciences (Küchemann, D., ed.), Vol. 13, pp. 81-135, Pergamon Press, 1972.

[23] Rasmussen, M. L., Jischke, M. C., and Daniel, D. C., "Experimental Forces and Moments on Cone-Derived Waveriders for $M_{\infty}=3$ to 5 ," Journal of Spucecraft and Rockets, Vol. 19, Nov.-Dec. 1982, pp. 592-598.

[24] Vanhoy, D., Low-speed Wind Tunnel Testing of a Mach 6 Viscous Optimized Waverider. Master's thesis, University of Maryland, College Park, 1988. 
[25] Corda, S., Vicsous Optimized Hypersonic Waveriders Designed From Flows Over Cones and Minimum Drug Bodies. PhD thesis, University of Maryland, College Park, 1988.

[26] Gillum, M. J., Analysis of Experimental Results on a Mach 14 Waverider. Master's thesis, University of Maryland, College Park, 1995.

[27] Corda, S., and Anderson, J. D., "Viscous Optimizod Hypersonic Waveriders Designed From Axisymnetric Flowficlds," AIAA Paper 88-0369, Jan. 1988.

[28] Cockrell, C. E., "Interpretation of Waverider Perfornance Data Using Computational Fluid Dynamics," AIAA Paper 93-2921, July 1993.

[29] He, X., and Rasmussen, M. L., "Computational Analysis of Off-Design Waveriders," Journal of Aircroft, Vol. 31, Mar.-Apr. 1994, pp. 345-353.

[30] Pegg, R. J., Hahne, D. E., and Cockrell, C. E., "Low-Specd Wind Tunnel Tests of Two Waverider Configuration Models," AIAA Paper 95-6093, Apr. 1995.

[31] Cockrell, C. E., Huebner, L. D., and Finley, D. B., "Aerodynamic Perforinance and Flow-Field Characteristics of Two Waverider-Derived Hypersonic Cruise Configurations," AIAA Paper 95-0736, Jan. 1995.

[32] Sobieczky, H., Dougherty, F., and Jones, K., "Hypersonice Waverider Dcsign from Given Shock Waves," in Proceedings of the First International Hypersonic Waverider Symposium, Oct. 1990.

[33] Millor, R. W., and Argrow, B. M., "Subsonic Aerodynamics of an Osculating Cones Waverider," AIAA Paper 97-0189, Jan. 1997.

[34] Grantz, A., "Calibration of Acrodynamic Engincering Methods for Waverider Design," AIAA Paper 94-0382, Janunry 1994. 
[35] Strohmeyer, D., Eggers, T., and Haupt, M., "Waverider Acrodynamics and Preliminary Design for Two-Stago-to-Orbit Missions, Part 1," Journal of Spacecraft and Rockets, Vol. 35, July-Aug. 1998, pp. 450-458.

[36] Strohmeyer, D., "Lateral Stability Derivatives for Osculating Cones Waveriders in Sub- and Transonic Flow," AIAA Paper 98-1618, $\Lambda$ pr. 1998.

[37] Starkey, R. P., and Lewis, M. J., "Analytical Off-Design Lift-to-Drag Ratio Analysis for Hypersonic Waveriders," Journal of Spacecraft and Rockets, Vol. 37, Sop.-Oct. 2000, pp. 684-691.

[38] Heiser, W. H., and Pratt, D. T., Hypersonic Airbrealling Propulsion. American Institute of Aeronautics and Astronautics, Inc., Washington, DC, 1994.

[39] Escher, W. J. D., and Flornes, B. J., "A Study of Composite Propulsion Systems for Advanced Launch Vehicle Applications," Final Report on NASA Contract NAS7-377, The Marquardt Corporation, Van Nuys, CA, Apr. 1967.

[40] Foster, R. W., Escher, W. J. D., and Robinson, J. W., "Air Augmented Rocket Propulsion Concepts," AFAL-TR-88-004, Apr. 1988.

[41] Iyde, E. H., Escher, W. J. D., and Roddy, J. E., "Marquardt's Mach 4.5 Supercharged Ejector Ramjet High-PerFormance Aircraft Engino Project," AIAA Paper 2000-3109, July 2000.

[42] Olds, J. R., and Bradford, J. E., "SCCREAM (Simulated Combined-Cycle Rocket Engine Analysis Module): A Conceptual RBCC Engine Design Tool," AIAA Parper 97-2760, 1997.

[43] Siebenhaar, A., and BuIman, M. J., "The Strutjet Engine: The Overlooked Option for Space Launch,", AIAA Paper 95-3124, July 1995. 
[4t] Taylor, G. I., and Maccoll, J. W., "The Nir Pressure on a Cone Moving at High Spect," Proc. Roy. Soc., Vol. 139, 1933, pp. 278-311.

[45] Kroyszig, E., Advanced Engineering Mathematics. John Wiley and Sons, Inc., Now York, 1993.

[40] Anderson, J. D., Modern Compressible Flow with Historical Perspective. McGraw-Hill, Inc., New York, 1990.

[47] Starkey, R. P., A Parametric Study of L/D and Volumetric Efficiency TradeOffs for Waverider Based Vehicles. Master's thesis, University of Maryland, College Park, 1998.

[48] Kee, R. J., Rupley, F. M., and Miller, J. A., "Chemkin-II: A Fortran Chemical Kinetics Package for the Analysis of Gas Phase Chemical Kinetics," SAND 89-8009B, April 1989.

[49] Anderson, J. D., Hypersonic and High Temperature Gas Dynamics. McGraw-Hill Book Company, New York, Now York, 1989.

[50] Larson, R., and Edwards, B., Elementury Linear Algebra. D. C. Heath and Company, Lexington, MA, 1991.

[51] Eckert, E. R. G., "Engineering Relations for Friction and Heat Transfor to Surfaces in High Velocity Flow," Journal of the Aeronautical Sciences, Vol. 22, 1955, pp. 585- 587.

[52] Ardema, M., Chambers, M., Terjesen, E., and Roberts, C., "Body Woight of Advanced Concept Hypersonic Aircraft," AIAA Paper 91-3180, 1991.

[53] Chuang, C., and Morimoto, H., "Periodic Optimal Cruise for a Ilypersonic Vehicle with Constraints," Journal of Spacecraft and Rockets, Vol. 34, Mar." Apr. 1997, pp. 165- 171. 
[54] McGrory, W., Slack, D., Applebaum, M., and Walters, R., GASP Version 3.0 Users Manual. Aerosoft, Inc., Blacksburg, Virginia, 1990.

[55] O'Bricn, T. F., Starkey, R. P., and Lewis, M. J., "A Quasi-One-Dimensional High-Speed Engine Model with Finitc-Rate Chemistry." submitted to Journal of Propulsion and Power, expected publication Sep--Oct. 2001.

[56] Shapiro, A. H., The Dynamics and Thermodynamics of Compressible Fluid Flow, Vol. 1. John Wilcy and Sons, New York, 1953.

[57] Turns, S. R., An Introduction to Combustion. McGraw-Hill, Inc, New York, New York, 1996.

[58] Gordon, S., and McBride, B. J., "Conputer Program for Calculation of Complex Chemical Equilibrium Compositions, Rocket Performance, Incident and Reflected Shocks and Chapman-Jouguct Detonations," NASA Report SP-273, 1971.

[59] Eckert, E. R. G., "Enginecring Relations for Heat Transfer and Friction in High-Velocity Laminar and Turlulent Boundary-Layer Flow over Surfaces with Constant Prcssure and Temperature," in Trunsactions of the ASME, Vol. 78, Aug. 1956.

[60] Byrne, G. D., Hindmarcl, A. C., and Brown, P. N., VODPK: VariableCoefficient Ordinary Differential Equation Solver with the Preconditioned Krylov Method GMRES for the Solution of Linear Systems. Lawrence Livcrmore National Laboratory, Livermorc, CA, 1997.

[61] Jachimowski, C. J., "An Analytical Study of the Hydrogen-Air Reaction witl Application to Scranjet Combustion," NASA TP 2791, Fobruary 1988.

[62] Rogers, R. C., "Mixing of Hydrogen Injected from Multiple Injectors Normal to a Supersonic Airstream," NASA TN D-6476, 1971. 
[63] Henry, J. R., and Anderson, G. X., "Design Considerations for the AirframeIntegrated Scramjet," NASA TM X-2895, 1973.

[64] Billig, F. S., and Grenleski, S. E., "Heat Transfer in Supersonic Combustion Processes," in Heat Transfer 1970 (Grigull, U., and Hahne, E., eds.), Vol. 3, pp. $1-11,1970$.

[65] Pinckncy, S. Z., "Turbulent Heat Transfer Prediction Method for Application to Scramjet Engines," NASA TN D-7810, November 1974.

[66] Anderson, G. Y., and Gooderum, P. B., "Exploratory Tests of Two Strut Fuel Injectors for Supersonic Combustion," NASA TN D-7581, 1974.

[67] Waltrup, P. J., and Billig, F. S., "Prediction of Precombustion Wall Prcssurc Distributions in Scramjet Engines," Journal of Spacerraft and Rockets, Vol. 10, Sep. 1973.

[68] Spreiter, J. R., and Alksne, A. Y., "Thin Airfoil Theory Based on Approximate Solution of the Transonic Flow Equation," NACA Report 1359, 1958.

[69] Spreiter, J. R., and Alksnc, A. Y., "Sletrder-Body Theory Based on Approximate Solution of the Transonic Flow Equation," NASA TR R-2, 1959.

[70] O'Bricn, T. F., and Lewis, M. J., "RBCC Engine-Airframe Integration on an Osculating Cone Waverider Vehicle," AIAA Paper 2000-3893, July 2000.

[71] Srinivasan, G. R., and Bacder, J. D., "TURNS: A Free Wake Euler / NavierStokes Numerical Method for Helicopter Rotors," AIAA Journal, Vol. 31, May 1993, pp. 959-962.

[72] Baldwin, B. S., and Lomax, H., "Thin Layer Approximation and Algebraic Model for Separated Turbulent Flows," AIAA Paper 78-0\%5\%, Jau. 1978. 
[73] Michel, R., Marchaud, F., and Le Gallo, J., "Ĺtude des écoulements transsoniques autour des profils lenticulaires, a incidence nulle," O.N.E.R.A. Pul. No. 65, 1953.

[74] Buning, P. G., Jespersen, D. C., Pulliam, T. H., Chan, W. M., Slotnick, J. P., Krist, S. E., and Renze, K. J., "Overllow User's Manual." NASA Langley Research Center, 1998.

[75] Taylor, R. A., and McDevitt, J. B., "Prossure Distributions at Tr"msonic Speeds for Parabolic-Arc Bodies of Revolution Having Fineness Ratios of 10,12 , and 14," NACA TN 4234, 1958.

[76] Chan, W. M., Chiu, I.-T., and Buning, P. G., "User's Manual for the HYPGEN Hyperbolic Grid Generator and the HGUI Graphical User Interface," NASA TM 108791, 1993.

[77] Shapiro, A. H., The Dynamics and Thermodynamics of Compressible Fluid Flow, Vol. 2. John Wiley and Sons, New York, 1953.

[78] Anderson, J. D., Fundamentals of Aerodynamics. McGraw-Hill Book Company, New York, New York, 1991.

[79] Anderson, J. D., A History of Aerodynamies. Cambridge University Press, Cambridge, United Kingdom, 1998.

[80] Hill, P. G., and Peterson, C. R., Mechanics and Thermodynamics of Propulsion. Addison-Wosley Publishing Company, Roading, Massachusetts, 1992. 